\title{
Forstplanung auf der Basis von Eingriffsinventuren
}

\author{
Dissertation zur Erlangung des Doktorgrades \\ der Fakultät für Forstwissenschaften und Waldökologie \\ der Georg-August-Universität Göttingen
}

vorgelegt von

Kai Staupendahl

geboren in Dortmund

Göttingen, im Juli 2006 
1. Gutachter: Prof. Dr. Dr. h.c. K. v. Gadow

2. Gutachter: Prof. Dr. J. Saborowski

3. Gutachter: Prof. Dr. H. Pretzsch

Tag der mündlichen Prüfung: 28.11.2006

Diese Arbeit ist durch die Niedersächsische Staats- und Universitätsbibliothek, SUBGöttingen, unter folgernder Internetadresse elektronisch veröffentlicht:

http://resolver.sub.uni-goettingen.de/purl/?webdoc-1628

bzw.:

http://webdoc.sub.gwdg.de/diss/2007/staupendahl/ 
„Der vor allem ist gut, der selber alles bedenket, Edel nenn ich auch den, der gutem Zuspruch gehorsam. Aber wer selber nicht denkt und auch dem Wissen des anderen Taub sein Herz verschließt, der Mann ist nichtig und unnütz.“ (Hesiod, Werke und Tage 293 und 295-7, nach v. Scheffer) 



\section{Vorwort}

Es ist wohl unwahrscheinlich, daß Hesiod (um 700 v. Chr.), als er die zuvor zitierten Verse schrieb, an Doktorarbeiten dachte. Trotzdem beschreiben Sie für mein Gefühl - vom etwas schroffen Urteil abgesehen - sehr schön das wissenschaftliche Arbeiten, wie ich es in den Jahren 1997 bis 2001, in denen diese Arbeit im wesentlichen entstand, am damaligen Institut für Forsteinrichtung und Ertragskunde kennen gelernt habe: Verstehen, würdigen und prüfen dessen, was andere zuvor gedacht haben und - ich gebe zu, der deutlich lustvollere Part - selber denken.

Eigenes Denken braucht Raum. Ich danke meinem Doktorvater, Herrn Prof. Dr. Dr. h.c. von Gadow, der mich bereits im Studium an die Fragen, Themen und Konzepte der Waldwachstumskunde, Forstplanung und Waldinventur heranführte und damit den Grundstein für diese Arbeit legte, besonders herzlich für die Freiheit, die er mir bei der Bearbeitung der gestellten Aufgaben gelassen hat, für sein konstantes Vertrauen und Zutrauen, seine Offenheit für meine Ideen, seine stetige Bereitschaft zum Gespräch und die oft entscheidenden Hilfestellungen, wenn die Arbeit ins Stocken geraten war. Dank der offenen Atmosphäre am Institut, dem regen internationalen Austausch, der Teilnahme an zahlreichen Tagungen im In- und Ausland und der Übertragung von Lehrverantwortung war es für mich eine prägende und lehrreiche Zeit, weit über die rein fachliche Ausbildung hinaus. Herrn Dr. Volker Stüber bin ich daher um so dankbarer dafür, daß er meine anfängliche Skepsis gegenüber dem Projekt Doktorarbeit kraft guter Argumente (,Du machst das doch gerne...“) zerstreuen konnte. Guten Zuspruch, nicht nur intellektueller Art, habe ich dabei auf vielerlei Weise erfahren:

Herrn Prof. Dr. Walter Zucchini, Herrn Prof. Dr. Joachim Saborowski, Herrn Prof. Dr. Alparslan Akça und Herrn Prof. Dr. Christoph Kleinn (der mich schon im Studium in Freiburg mit den Gesetzen der Biometrie bekannt machte) danke ich für ihren immer wieder gerne gegebenen Rat in Fragen der Statistik und Waldinventur, durch den ich viel lernen konnte und der sicherlich wesentlich dazu beigetragen hat, daß diese Arbeit eine wissenschaftliche genannt werden darf. Besonders Prof. Akça verstand daneben durch seinen herzlichen Humor den Arbeitsalltag eines manchmal an eben diesen Problemen der Statistik und Inventur sich festbeißenden Doktoranden aufzulockern.

Ohne Daten keine Statistik. Daß ich diesbezüglich nie auf dem Trockenen saß, verdanke ich neben Herrn Hendrik Heydecke, der mir die Versuchsflächendaten des Institutes zugänglich machte, und allen Doktoranden, Diplomanden und Mitarbeitern, die sie einst erhoben hatten, Herrn Prof. Dr. Hermann Spellmann und Herrn Prof. Dr. Jürgen Nagel, die mir für die Modellierung der Durchforstungen umfangreiche Datensätze aus dem Versuchsflächennetz der Nordwestdeutschen Forstlichen Versuchsanstalt zur Verfügung stellten und mich immer wieder mit ihrem fachlichen Rat unterstützen. Entgegen mancher Gerüchte über modellorientierte Forstwissenschaftler war ich zur Datenerhebung und zum praktischen Test der Inven- 
turverfahren aber auch selber im Wald, und zwar (ganz überwiegend) mit allergrößtem Vergnügen. Das verdanke ich nicht zuletzt Justus v. Geibler, Jörg Schröder, Reinhard Schlote, Christian Roschak und Pirrka Spanuth, die mich dabei tatkräftig unterstützt haben.

„Du wirst nie zuhause sein, wenn Du keinen Gast, keine Freunde hast", singt Heinz Rudolf Kunze. Vor allem meinen Kollegen und Freunden (in order of appearance) Dr. Matthias Schmidt, Dr. Jörg Schröder, Dr. Matthias Albert, Stefan Daume, Dr. Janna Puumalainen, Pirrka Spanuth, Justus v. Geibler, Dr. František Vilčko, Dr. Torsten Vor, Dr. Lars Hinrichs und Dr. Lutz Fehrmann danke ich für manches hocheffektive ,informelle“ Fachgespräch, für nakken- und gesichtsmuskulaturlockernde Dart-Runden und andere sportliche Einlagen, für gemeinsam gelöste Fälle im „Kommissariat“ und viele schöne Stunden innerhalb und außerhalb des Institutes. Ohne sie wäre diese Arbeit ein Zeugnis traurigen Eremitentums. Besonders herzlich danken möchte ich Justus v. Geibler und Dr. Matthias Schmidt für ihre Hilfe und Unterstützung in der letzten heißen Phase vor Abgabe der Arbeit. Wenn der Leser den roten Faden erkennen kann, dann nicht zuletzt dank ihrer von Betriebsblindheit ungetrübten Hinweise.

Ohne Moos nichts los. Und manchmal auch nicht ohne Messgerät. Oder ohne Briefmarke. Daß es mir an nichts von alledem gemangelt hat und für ihre stetige freundliche Hilfsbereitschaft, dafür danke ich den festen Instituts-Mitarbeitern und Mitarbeiterinnen Herrn Hendrik Heydecke, Frau Ulrike Dockter, Herrn Reinhard Schlote, Frau Sonja Rüdiger und Frau Margret Krüger. Wenn ich mich in den Untiefen der Programmierung und Betriebssystemfunktionen manchmal schon verloren glaubte, war es oft Hendrik Heydecke, der mir den entscheidenden Tipp gab. Ohne diesen Austausch unter Leidensbrüdern und die vielen guten Gespräche über Gott und die Welt (des Fußballs) wäre so mancher Tag um einiges ärmer gewesen. Besonders danke ich auch Ulrike Dockter, die mich von administrativen Arbeiten weitgehend entlastet und oft mit großem persönlichem Einsatz dafür gesorgt hat (also fürsorglich im wahrsten Wortsinn), daß mir die verfügbaren Finanzmittel auch tatsächlich zur Verfügung standen.

Meinen Eltern, Dres. med. Gisela und Dieter Staupendahl, und meinen Geschwistern Frank und Frauke Staupendahl danke ich zuletzt und doch an erster Stelle: Sie haben mich liebend begleitet, ermutigt, gestärkt und gefördert. Ohne sie ist diese Arbeit nicht denkbar. 



\section{Publikationen}

Die vorliegende Arbeit ist eine Zusammenfassung der folgenden Publikationen:

I StAupendahl, K., und GAdOW, K. v., 2008: Eingriffsinventuren und dynamisches Betriebswerk - Instrumente der operativen Planung im Forstbetrieb. Forstarchiv 79 (im Druck).

II StAuPENDAHL, K., 2008: Die modifizierte 6-Baum-Stichprobe - Ein geeignetes Verfahren zur Erfassung von Waldbeständen. Allg. Forst- u. Jagdztg. 179 (im Druck).

III Staupendahl, K., und Zucchini, W., 2006: Estimating the spatial distribution in forest stands by counting small angles between nearest neighbours. Allg. Forst- $u$. Jagdztg. 177 (8/9): 160 - 168.

IV Staupendahl, K., 1999: Modelling thinnings based on the ratio of relative removal rates. In: PUKKALA, T., and EERIKÄINEN, K. (eds.): Growth and yield modelling of tree plantations in South and East Africa - Proceedings of the meeting in Mombassa, Kenya, 12.-15. Oct. 1999. The Univ. of Joensuu, Faculty of Forestry Res. Notes 97: $183-194$.

Die Arbeit bezieht sich auf die einzelnen Publikationen unter Angabe der jeweiligen römischen Ziffer (I - IV). 


\section{Inhaltsverzeichnis}

1 Einleitung 1

1.1 Bestandes- und Betriebsinventuren als konkurrierende Verfahren der forstlichen Planung 1

1.2 Eingriffsinventuren als Verfahren zur bestandesweisen Zustandserfassung 3

1.3 Zielsetzung und Schwerpunkte der Arbeit 5

2 Das Prinzip der Eingriffsinventur $\quad 7$

2.1 Getrennte und bedarfsorientierte Erfassung des ausscheidenden und verbleibenden Bestandes

2.2 Fortschreibung der Bestandesdaten $\quad 8$

3 Erfassung des ausscheidenden Bestandes $\quad 10$

3.1 Vollkluppung im Zuge des Auszeichnens $\quad 10$

3.2 Schätzung der Durchmesserverteilung mit Hilfe der Weibullfunktion 10

4 Erfassung des verbleibenden Bestandes $\quad 16$

4.1 Auswahl des Inventurverfahrens 16

4.2 Inventurintervall 18

4.3 Die modifizierte 6-Baum-Stichprobe 21

4.3.1 Das punktbezogene Winkelmaß 22

4.3.2 Schätzung der Stammzahl und Grundfläche pro Hektar 27

4.3.3 Aufnahmetechnische Modifikationen $\quad 32$

$\begin{array}{ll}\text { 4.3.4 Validierung } & 35\end{array}$

5 Software zur Fortschreibung, Planung und Entscheidungsunterstützung 39

6 Diskussion $\quad 42$

7 Zusammenfassung $\quad 45$

8 Literatur $\quad 48$

$\begin{array}{ll}\text { Anhang } & 55\end{array}$

$\begin{array}{ll}\text { Publikation I } & 57\end{array}$

$\begin{array}{ll}\text { Publikation II } & 91\end{array}$

$\begin{array}{lr}\text { Publikation III } & 123\end{array}$

$\begin{array}{ll}\text { Publikation VI } & 145\end{array}$ 



\section{Einleitung}

\subsection{Bestandes- und Betriebsinventuren als konkurrierende Verfahren der forst- lichen Planung}

Die Erweiterung des bisherigen Verständnisses von Nachhaltigkeit (z. B. MCPFE 2000) und der stetig wachsende Anteil von strukturreichen Mischwäldern stellen die Forstplanung vor neue Aufgaben. Die in gleichaltrigen Reinbeständen dank der Ertragstafeln relativ unkomplizierte Planung und Nachhaltskontrolle (KRAMER 1990) ist in ungleichaltrigen Mischbeständen nur dann möglich, wenn es mit neuen Hilfsmitteln gelingt, die vielfältigen Funktionen der Ertragstafel zu ersetzen. Dabei ist zu beachten, daß der mit einem ganzheitlichen Nachhaltsbegriff einhergehende erweiterte Bedarf an Daten über die Waldstruktur (POMMERENING und GADOW 2000) neue Inventurmethoden erforderlich macht.

Auch die Änderung der ökonomischen Rahmenbedingungen stellen eine Herausforderung dar. So werden z. B. im Ergebnisbericht einer vom schweizerischen Bundesamtes für Umwelt in Auftrag gegebenen Studie (ProgrammLeitung holz21 2003) steigende Kosten, abnehmende Erträge, Überkapazitäten entlang der Wertschöpfungskette und ein wachsender Schnittholzimportdruck innerhalb der mitteleuropäischen Wald- und Holzwirtschaft konstatiert. Diese veränderten Rahmenbedingungen erfordern die Weiterentwicklung der Forsteinrichtung zu einer umfassenden Waldplanung, eine Aufgabe, die umso anspruchsvoller ist, je unsicherer die Holzmarktentwicklung, je ungleichförmiger die Waldstrukturen, je größer die Vielfalt der waldbaulichen Vorstellungen und je restriktiver die Vorgaben zur Kostensenkung sind.

Grundlage einer auf Nachhaltigkeit bedachten Forstplanung sind verläßliche Informationen über Holzvorräte, Wert und Struktur des bewirtschafteten Waldes. In den bisher vorherrschenden Altersklassenwäldern wurden diese Daten mit Hilfe periodischer Bestandesinventuren ermittelt, wobei der sogenannte Waldbegang mit Ertragstafelschätzung das überwiegend angewandte Verfahren war (KRAMER und AKÇA 1995, S. 163 f.). Die in den letzten Jahren bis Jahrzehnten vielfach praktizierte Zielstärkennutzung mit verlängerten Verjüngungszeiträumen (z. B. Отто 1992) hat im Zusammenwirken mit veränderten Wachstumsbedingungen und Durchforstungskonzepten dazu geführt, daß das Ertragstafelkonzept auf großer Fläche nicht mehr sinnvoll anwendbar ist. Bedingt durch höhere Artenvielfalt und Ungleichaltrigkeit entstehen in naturnah bewirtschafteten Forstbetrieben immer komplexere Waldstrukturen. Dadurch hat die Betriebsinventur (Kontrollstichprobe) als alternatives Inventurkonzept zunehmend an Bedeutung gewonnen (SCHMID-HAAS 1969a, 1989; WEIDENBACH und KARIUS 1993; AKÇA 1993).

Beispiele für Betriebsinventuren sind die Verfahren der Landesforstbetriebe bzw. -verwaltungen Niedersachsen (BÖCKMANN et al. 1998a, 1998b), Bayern (FUCHS und KENNEL 1994) und Baden-Württemberg (HINRICHS et al. 1993; TEUFFEL und KREBS 1999). Sie ermöglichen die Schätzung von metrischen Variablen für sogenannte Befundeinheiten bzw. Straten, 
und ihre Vorteile liegen insbesondere in der Objektivierung der Erfassung des Waldzustandes, einer statistisch abgesicherten Beschreibung der mittel- bis langfristigen Waldentwicklung (bei vorliegenden Wiederholungsinventuren) und relativ flexiblen Auswertungsmöglichkeiten innerhalb der Straten und auf der Ebene des Gesamtbetriebes.

Diesen Vorteilen stehen jedoch einige Nachteile gegenüber (GADOW und SCHMIDT 1998). So lassen sich die Daten aus Betriebsinventuren z. B. kaum für die operative Planung nutzen, was u. a. daran liegt, daß keine bestandesbezogenen Daten erhoben werden, da ein Bestand i.d.R. zu wenig (oder gar keine) Probekreise enthält und somit die in den Probekreisen enthaltenen Informationen nicht ohne weiteres auf die Bestandesebene übertragen werden können. Zur Lösung dieses Problems der Übertragung von punktbezogenen Informationen in die Fläche (Regionalisierung) sind mittlerweile einige Ansätze vorgestellt worden. Sie reichen von der einfachen gutachtlichen Schätzung (z. B. BöCKMANN et al. 1998b) bis zu Methoden der räumlichen Statistik (vgl. GÜSSEFELDT 1997), wobei dem Einsatz von Luftbildern und Satellitendaten eine besondere Bedeutung zukommt (z. B. MUINONEN et al. 2001; STÜMER 2004; NIESCHULZE et al. 2005). Die Ergebnisse sind allerdings nur teilweise zufriedenstellend und erfordern natürlich zusätzlichen Mitteleinsatz. Inwieweit dabei der Nutzen in einem günstigen Verhältnis zu den Kosten steht, wurde bisher nicht untersucht.

Wenn die Regionalisierung der Probekreisdaten nur unvollständig möglich ist, erhält man also genaue Informationen über die Holzvorräte, aber nur sehr ungenaue über deren Örtlichkeit. Dieser Raumbezug, der für die Optimierung, Planung und Durchführung aller waldbaulichen Maßnahmen entscheidend ist, kann nur über den Bestand als topologisch eindeutig definierte Buchungs- und Kontrolleinheit gewährleistet werden. Seine Bedeutung kommt auch in der Anwendung geographischer Informationssysteme zum Ausdruck, die zunehmend zur Optimierung der Holzernte und Transportprozesse genutzt werden (z. B. GADOW und Chen 2002; WALTER und CARLSSON 1998).

Da mit der Betriebsinventur eine ausreichend genaue Zustandsbeschreibung auf Bestandesebene nur selten möglich ist, werden natürlich auch die Durchforstungen und Erntemaßnahmen nur für die einzelnen Straten geplant, wobei man sich bei der Festlegung von Eingriffsart, -stärke und -zeitpunkt an einem idealtypischen Durchforstungsschema sowie den durchschnittlichen Verhältnissen orientiert (vgl. SPELLMANN et al. 1999). Während ein solches Verfahren für die Planung der insgesamt anfallenden Nutzungsmassen rationell und kostensparend ist, liefert es für das waldbauliche Vorgehen in einem konkreten Bestand kaum Entscheidungshilfen, obwohl vor dem Hintergrund anspruchsvoller Waldbaukonzepte Unsicherheiten hinsichtlich der „richtigen“ Pflege- und Nutzungsentscheidungen bestehen. Zur Verbesserung der Entscheidungsgrundlagen ist die Zielvereinbarung anhand von Weiserflächen (z. B. BÖCKMANN et al. 1998b) allein nicht ausreichend, da diese immer nur Bestandestypen repräsentieren, von denen konkrete Einzelbestände in ihrem Aufbau und Zustand mehr oder weniger abweichen. 
Durch die Entkoppelung der Bewirtschaftungseinheit Waldbestand von den Planungsprozessen Vorratschätzung, Nutzungsplanung und Fortschreibung nimmt der Wert der bereits zu Anfang der Planungsperiode ungenauen Bestandesdaten mit der Länge des Fortschreibungszeitraumes immer mehr ab, und zwar um so stärker, je öfter durchforstet wird und je stärker die tatsächlichen von den unterstellten Durchforstungen abweichen. Zudem wird die Prognose der weiteren Bestandesentwicklung verfälscht sein, wenn durch die geschätzten Durchforstungen deutlich abweichende Wuchskonstellationen erzeugt werden (z. B. PRETZSCH 1995, 1997; POMMERENING 1998). Neben der abnehmenden Aktualität der Bestandesdaten läßt sich überdies die Zielkonformität der realisierten Eingriffe erst im Nachhinein beurteilen. Für die zeitnahe Kontrolle der Produktion ist die Betriebsinventur daher kaum geeignet.

Es soll an dieser Stelle auch daran erinnert werden, daß die Kontrollstichprobe primär für die speziellen Verhältnisse der „homogen inhomogenen“ Schweizer Plenterwälder entwickelt wurde, also für Forstbetriebe, die praktisch nur durch einen einzigen Bestand gebildet werden. Hier liefert sie selbstverständlich operationale Informationen und verbessert die Kontrolle der waldbaulichen Maßnahmen. Anders in Betrieben, die sich aus einer Vielzahl von Beständen mit unterschiedlichsten Strukturen zusammensetzen, wie es in Deutschland ganz überwiegend der Fall ist (GADOW und SCHMIDT 1998). Daß sich dieser Zustand grundlegend ändern wird, darf bezweifelt werden, da vor dem Hintergrund des teilweise drastischen Personalabbaus der letzten $10 \mathrm{Jahre}^{1}$ und der zunehmenden Ausrichtung auch der öffentlichen Forstbetriebe auf ökonomischen Erfolg (HOSTETTLER 2006) sich in den letzten Jahren wieder Tendenzen zu einem großflächigeren Wirtschaften feststellen lassen (z. B. HocKENJOS 2006), die der eingangs skizzierten Entwicklung hin zu einer Auflösung der Bestandesgrenzen zuwiderlaufen.

\subsection{Eingriffsinventuren als Verfahren zur bestandesweisen Zustandserfassung}

Da waldbauliche Maßnahmen immer auf der Ebene des Einzelbestandes ansetzen, können nur räumlich definierte Informationen zu deren Optimierung beitragen. Zudem muß die Örtlichkeit der Holzvorräte bekannt sein, um flexibel auf die Anforderungen des Marktes reagieren zu können. Daher sollten nach Tzschupke (1991), SAgL (1995) und BitTer und Merrem (1998) auch künftig bestandesbezogene Inventuren die erforderlichen Informationen für die mittelfristige Planung liefern. Dies gelte um so mehr, als auch viele Schutz- und Erholungsleistungen des Waldes, die neben der Holzproduktion erbracht werden, primär über die jeweilige Fläche wirksam werden. Bestandesbezogene Inventur- und Planungsansätze besitzen

\footnotetext{
${ }^{1}$ So werden z. B. in den Niedersächsischen Landesforsten im Vergleich zum Stand vor 1997 bis zum Jahr 2008 $40 \%$ der Beamten, Angestellten und Forstwirte eingespart worden sein (MERKER 2006)
} 
somit den Vorzug, daß sie grundsätzlich die Möglichkeit bieten, die strategische und die operationale Ebene miteinander zu verknüpfen (s. z. B. GADOW und PUUMALAINEN 2000).

Als Alternative zu den periodischen Inventurverfahren ist daher am Institut für Waldinventur und Waldwachstum der Georg-August-Universität Göttingen das Konzept der bestandesbezogenen Eingriffsinventur aufgegriffen und weiterentwickelt worden. Wie der Name bereits sagt, werden solche Inventuren nicht in fest definierten Zeitabständen durchgeführt, sondern immer dann, wenn ein Eingriff bevorsteht (GADOW und STÜBER 1994). Die Aufnahme des Bestandes basiert auf einer temporären, systematischen Stichprobe und erfolgt nach dem Auszeichnen, aber vor der Entnahme der markierten Bäume. Die Vorteile dieses Vorgehens wurden von GADOW und SCHMIDT (1998) ausführlich beschrieben. Im Kontext dieser Arbeit sind folgende Punkte von besonderer Bedeutung:

- Eine Bestandesinventur ermöglicht eine flexible Ausrichtung des Inventuraufwandes am Informationsbedarf und damit eine Optimierung des Kosten-Nutzen-Verhältnisses der Inventuren. So kann durch eine Extensivierung der Datenerhebung in jungen und strukturarmen Beständen die Kostensteigerung durch eine intensivierte Informationsgewinnung in wertvollen Beständen kompensiert werden.

- Durch die gleichzeitige Erfassung des Bestandeszustandes und dessen Veränderung kann die waldbauliche Maßnahme analysiert und gegebenenfalls korrigiert werden (präventive Nachhaltskontrolle).

- Die Zustandsgrößen des verbleibenden Bestandes verändern sich bis zur nächsten Inventur - sofern keine Kalamitäten auftreten - nur durch das natürliche Wachstum und die Mortalität der Bäume, also Prozesse, die inzwischen selbst für ungleichaltrige Mischbestände ziemlich genau vorhergesagt werden können (STERBA 1990; HASENAUER et al. 1995; Nagel 1999, 2000; Pretzsch und Kahn 1996; Pretzsch und Dursky 2001; HALLENBARTER et al. 2005).

- In Verbindung mit einer jährlichen, bestandesweisen Aktualisierung der Holzvorratsdaten, basierend auf einer Zuwachsschätzung mit Hilfe von Wuchsmodellen (Fortschreibung), bleibt die Aktualität der Daten über den gesamten Planungszeitraum erhalten.

Aus dem oben gesagten wird deutlich, daß der nach Brusthöhendurchmesserklassen differenzierten Erfassung des ausscheidenden Bestandes eine zentrale Rolle bei der Pflege der Forsteinrichtungsdaten zukommt. Zugleich dient sie der betrieblichen Steuerung und der Optimierung von Holzernte und -absatz (z. B. PuUmalainen 1998, S. 3 f.). Mögliche Anwendungsbereiche sind z. B. (vgl. RHEINLAND-PFÄLZISCHES FORSTAMT DAUN 2000):

- Optimierung der Sortenaushaltung und der Arbeitsverfahren

- Optimierung der Zuordnung von Lieferaufträgen zu Hieben und Optimierung der Blockbildung bei der Maßnahmenplanung 
- Aufstellung von zuverlässigen Hiebs-, Verkaufs- und Finanzplänen

- Möglichkeit des Stockverkaufs mit differenzierter Preisbildung und Rechnungsausstellung vor Aufarbeitungsbeginn

- Verbesserte Ausschreibung und Vergabe von Lohnunternehmeraufträgen

Die Erfassung des ausscheidenden Bestandes, die Weiterverarbeitung dieser Daten und ihre Integration in den vorhandenen Datenbestand ist damit ein wichtiger Bestandteil der (forstbetrieblichen) Logistik, die nach SCHÖNSLEBEN (2000) die „Organisation, Planung, Realisierung und Steuerung des gesamten Informations- und Materialflusses entlang der ganzen Wertschöpfungskette“ bezeichnet. HeINIMANN (1999) weist darauf hin, daß die Wandlung des Holzmarktes von einem Verkäufer- zu einem Käufermarkt Produktionsprozesse erfordert, welche sich viel stärker als bisher an den Bedürfnissen des Marktes orientieren. Herzstück einer kundenorientierten Rohholzbereitstellung sollte daher die Auftragsliste sein. Die zur Optimierung eines solchen Produktionsprozesses erforderlichen Informationen über die Sortenzusammensetzung der geplanten Einschlagsvolumina können nur mit einer vor der Nutzung stattfindenden Erfassung oder Schätzung der Durchmesserverteilung des ausscheidenden Bestandes bereitgestellt werden.

\subsection{Zielsetzung und Schwerpunkte der Arbeit}

Vor diesem Hintergrund soll ein Konzept der permanenten Inventur entwickelt werden, das in die betrieblichen Abläufe und Informationsflüsse vollständig integriert werden kann, eine flexible Anpassung an den tatsächlichen Informationsbedarf erlaubt und über die Mehrfachnutzung der erhoben Daten sowohl der Zustandserfassung im Rahmen der Forsteinrichtung als auch dem forstbetrieblichen Controlling und der Optimierung von Produktion und Absatz dient. Um dieses Ziel zu erreichen, werden neben der Entwicklung eines schlüssigen Konzeptes, basierend auf den bestehenden Verfahren der Forsteinrichtung, folgende Schwerpunkte bearbeitet:

- Erfassung des ausscheidenden Bestandes. Hier gilt es insbesondere für die Fälle, in denen eine Inventur zu aufwendig wäre, eine Methode zur Schätzung der Durchmesserverteilung des ausscheidenden Bestandes zu entwickeln (Kap. 3.2).

- Erfassung des verbleibenden Bestandes. Zentrale Fragen sind hierbei die Wahl der Inventurmethode (Kap. 4.1) und des Inventurintervalls (Kap. 4.2). Für den Fall einer Stichprobeninventur wird aufgrund ihrer Praktikabilität die 6-Baum-Stichprobe vorgeschlagen. Zur Korrektur des dieser Methode inhärenten systematischen Fehlers der Stammzahl- und Grundflächenschätzung werden Methoden erarbeitet, die zum einen auf der Beschreibung des räumlichen Baumverteilungsmusters mit Hilfe eines Aggregationsindexes (Kap. 4.3.1 u. 4.3.2), zum anderen auf einer Modifikation des Verfahrens (Kap. 4.3.3) beruhen. 
- Entwicklung eines Softwaresystems. Hierbei werden die Softwarekomponenten beschrieben, die eine optimale Nutzung der Inventurdaten im Sinne eines Systems zur Entscheidungsunterstützung und die Fortschreibung der Holzvorräte erlauben (Kap. 5).

In Kap. 6 wird das vorgestellte Konzept schließlich unter dem Kosten-/Nutzen-Aspekt und hinsichtlich seiner Konsequenzen für die Organisation der Forsteinrichtung diskutiert. 


\section{Das Prinzip der Eingriffsinventur}

\subsection{Getrennte und bedarfsorientierte Erfassung des ausscheidenden und verblei- benden Bestandes}

In dem von GADOW und STÜBER (1994) und GADOW und SCHMIDT (1998) vorgestellten Verfahren ist die gleichzeitige Erfassung des ausscheidenden und verbleibenden Bestandes durch eine Stichprobeninventur nach dem Auszeichnen und vor der Ausführung des Eingriffs vorgesehen. Die Vorteile, die sich aus diesem Vorgehen ergeben, wurden bereits erläutert. Ein besonders wichtiger Aspekt ist in diesem Zusammenhang die Beurteilung der eingriffsbedingten Veränderung von Vorrat, Struktur und Wert. Durch die gleichzeitige Erfassung von verbleibendem und ausscheidendem Bestand verhält sich eine solche Inventur bezüglich der Zustandsveränderung wie eine permanente Stichprobe, deren Vorteil darin besteht, daß sie die Änderungsrate mit einem geringeren Fehler schätzt als temporäre Stichproben (AKÇA 2001, S. 131). Gleichzeitig ergeben sich aber auch nicht unwesentliche Nachteile:

- Da in der Vornutzung nur sehr selten mehr als $15 \%$ des Vorrats entnommen werden, wird der ausscheidende Bestand i.d.R. mit einem nur kleinen Anteil in die Stichprobe eingehen. Dadurch ist der Stichprobenfehler für dieses Kollektiv relativ hoch, was den Nutzen dieser Art der Datenerhebung in Frage stellt.

- Der betriebliche Organisations- und Abstimmungsaufwand ist durch das enge Zeitfenster, innerhalb dessen die Inventur der Bestände erfolgen muß, recht hoch. Unter der Annahme, daß der Umfang der „Just-in-time“-Holzernte in Zukunft weiter zunehmen wird, ist es wahrscheinlich, daß die Durchführung der Inventur in vielen Fällen nicht rechtzeitig möglich sein wird.

Aus diesen Gründen wird eine zeitlich getrennte Erfassung von verbleibendem und ausscheidendem Bestand vorgeschlagen (s. Abb. 1).

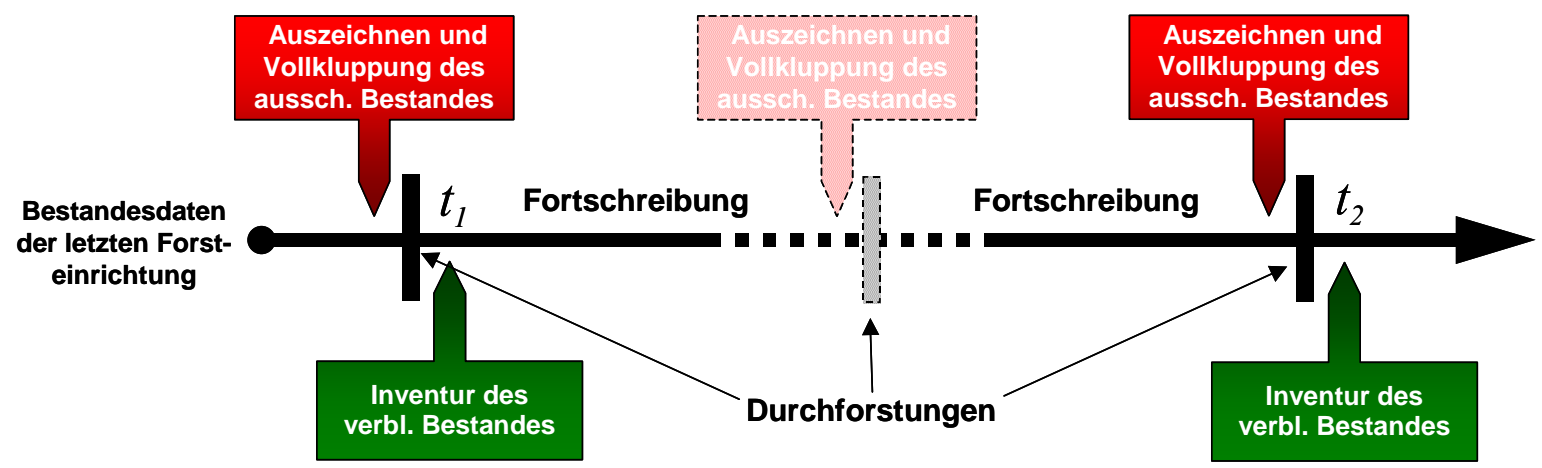

Abb. 1. Ablaufschema der Eingriffsinventuren entlang der Zeitachse. Die vertikalen Balken markieren den Zeitpunkt der Hiebsmaßnahmen. Das Intervall zwischen den Inventuren des verbleibenden Bestandes ist bestandesindividuell und sollte in Abhängigkeit von der Wuchsdynamik und Durchforstungshäufigkeit zwischen 15 und 25 Jahren liegen (s. a. Kap. 4.2). Die Fortschreibung zwischen den Inventuren erfolgt mit Hilfe eines Wuchsmodells (vgl. NAGEL 1998). 
Zur Erfassung des ausscheidenden Bestandes sollte während des Auszeichnens jeder zu entnehmende Baum auch gekluppt werden. Diese Vollaufnahme erfordert nur geringen Mehraufwand, wenn hierfür eine elektronische Kluppe eingesetzt wird, mit der jeder Durchmesser getrennt nach Baumart gespeichert und später über eine geeignete Schnittstelle auf den PC übertragen werden kann. Die baumartenspezifischen Durchmesserverteilungen des ausscheidenden Bestandes werden auf diese Weise - solange keine Meßfehler auftreten - absolut zuverlässig und ohne jeden Stichprobenfehler erfaßt. Werden auch die baumbezogenen Mittelhöhen gemessen und die Einzelbaumhöhen über Einheitshöhenkurven geschätzt, stehen somit sehr genaue Daten zur Verfügung, die für vielfältige Zwecke genutzt werden können (s. Kap. 1.2). Für den Fall, daß eine Vollkluppung des ausscheidenden Bestandes (z. B. in sehr jungen und stammzahlreichen Beständen) nicht möglich oder sinnvoll ist, werden alternative Verfahren der Erfassung in Kap. 3 vorgestellt.

Falls für den verbleibenden Bestand keine Daten vorliegen oder die vorhandenen Daten veraltet sind (s. a. Kap. 4.1), erfolgt nach dem Einschlag dessen Neuinventur. Wenn diese auf einem Stichprobenverfahren basiert, wird im Vergleich mit der Methode von GADOw und STÜBER (1994) für jeden nicht aufgenommenen ausscheidenden Baum ein zusätzlicher Baum des verbleibenden Bestandes in die Stichprobe eingehen, so daß dieser genauer erfaßt wird bzw. die geforderte Genauigkeit mit einem geringeren Inventuraufwand eingehalten werden kann. Dies ist von einiger Bedeutung für die Fortschreibung der Bestandesdaten, da die Qualität der Prognose selbstverständlich nicht nur von der Validität der verwendeten Wuchsmodelle, sondern mindestens ebenso von der Genauigkeit der Zustandserfassung abhängt. Aus Gründen der besseren Begehbarkeit des Bestandes ist die Durchführung der Inventuren vor dem Einschlag natürlich wünschenswert. Allerdings müssen dann die zur Nutzung vorgesehen Bäume bereits markiert sein, damit die alleinige Erfassung des verbleibenden Bestandes möglich ist. Und selbst dann besteht natürlich die Gefahr, daß später nicht genau die markierten Bäume entnommen werden.

\subsection{Fortschreibung der Bestandesdaten}

Die Daten des verbleibenden Bestandes werden nun jedes Jahr bis zur nächsten Inventur mit Hilfe eines Wachstumssimulators fortgeschrieben (vgl. z. B. NAGEL 1998; POMMERENING 1998). Zwischenzeitlich stattfindende Eingriffe werden wie beschrieben durch Vollkluppung während des Auszeichnens (oder alternative Verfahren) erfaßt und in die Datenbank importiert. Die Durchmesserverteilungen des ausscheidenden Bestandes werden nach Darstellung am Bildschirm von den fortgeschriebenen Durchmesserverteilungen subtrahiert und der Datenbestand dadurch aktualisiert. Die hieraus resultierenden Daten werden anschließend weiter fortgeschrieben. Auf diese Weise werden mehrere Zwecke erfüllt: 
- Jeder Eingriff kann ziemlich genau analysiert werden. Der auszeichnende Förster erhält dadurch objektive Informationen über die Auswirkungen seines waldbaulichen Vorgehens und kann es (falls der ausscheidende Bestand vor dem Eingriff erfaßt wurde) gegebenenfalls korrigieren.

- Es stehen jederzeit aktuelle Zustandsdaten der Bestände und damit des Forstbetriebes zur Verfügung.

- Die Erfassung des ausscheidenden Bestandes dient nicht nur der Optimierung von Holzernte und Absatz, sondern zugleich der Aktualisierung und Fortschreibung der Bestandesdaten. Umgekehrt können Daten aus der Inventur des verbleibenden Bestandes für die Kalkulation der anfallenden Sortimente genutzt werden: Unter der Vorraussetzung, daß sich das durchschnittliche H/D-Verhältnis der Bäume im verbleibenden und ausscheidenden Bestand nicht wesentlich unterscheidet, könnte z. B. die zusätzliche Messung von Baumhöhen entfallen, da diese über die Bestandeshöhenkurve des verbleibenden Bestandes geschätzt werden könnten (s. Kap. 4.3).

- Durch die langfristige Aufzeichnung der Bestandesentwicklung entsteht ein wertvoller Datenfundus, der für vielfältige Zwecke (z. B. die lokale Kalibrierung der verwendeten Wuchsmodelle) genutzt werden kann.

Durch diese Mehrfachnutzung der Daten wird die Wirtschaftlichkeit der Inventuren erheblich gesteigert. 


\section{Erfassung des ausscheidenden Bestandes}

\subsection{Vollkluppung im Zuge des Auszeichnens}

Wie bereits in Kap. 2.1 beschrieben wurde, stellt die Vollaufnahme der Durchmesserverteilung des ausscheidenden Bestandes durch Kluppen jedes markierten Baumes eine sehr genaue und dennoch wenig aufwendige Methode dar. Ein in der Praxis bereits bewährtes Arbeitsverfahren, das ebenfalls auf der vollständigen Erfassung der Durchmesserverteilung der zu entnehmenden Bäume beruht, wurde durch das RHEINLAND-PFÄLZISCHE FORSTAMT DAUN (2000) vorgestellt. Die bei diesem Verfahren angestrebte genaue Schätzung der anfallenden Sortimente mit Hilfe des Programms Holzernte (SCHÖPFER 1998) macht die zusätzliche Messung von ca. 20 - 40 Baumhöhen zur Erstellung einer bestandesindividuellen Höhenkurve erforderlich. Allerdings sollte geprüft werden, ob die Verwendung einer Einheitshöhenkurve nicht eine ausreichende Genauigkeit liefert. Hierdurch ließe sich die Anzahl der arbeitsintensiven Höhenmessungen deutlich reduzieren, da zur Ermittlung der dann benötigten Bestandesmittelhöhe nur 5 - 10 Baumhöhen gemessen werden müßten (vgl. a. NIEUWENHUIS et al. 2001; UUSiTALO 1995; UUSITALO und KIVINEN 2000),

Ist die Vollkluppung des ausscheidenden Bestandes (z. B. in sehr jungen und stammzahlreichen Beständen) nicht möglich oder sinnvoll, kann deren Durchmesserverteilung geschätzt werden. Hierfür stehen zwei alternative Methoden zur Verfügung, die allerdings keine vorherige Kontrolle des Eingriffs erlauben: Wurde der Bestand mit dem Harvester aufgearbeitet, kann die Durchmesserverteilung der entnommenen Bäume aus den im Bordcomputer gespeicherten Daten ohne großen Aufwand generiert werden. Bei motormanueller Durchforstung kann die Schätzung mit Hilfe des im folgenden beschriebenen, auf der Weibullfunktion basierenden Ansatzes erfolgen (vgl. a. STAUPENDAHL und PUUMALAINEN 1999).

\subsection{Schätzung der Durchmesserverteilung mit Hilfe der Weibullfunktion}

Um die hier gestellten Anforderungen an ein Durchforstungsmodell erfüllen zu können, war es v. a. wichtig, die Art und Stärke einer Durchforstung quantitativ, aber dennoch anschaulich und einfach zu beschreiben. Da in der Praxis ein weites Spektrum von Durchforstungsarten zu erwarten ist, sollte das Modell diese möglichst flexibel abbilden können. Mit den Durchmesserverteilungen von 67 Buchenreinbeständen (ausscheidend und verbleibend) aus 9 langfristigen Durchforstungsversuchen der Nordwestdeutschen Forstlichen Versuchsanstalt wurde zur Parametrisierung deshalb entsprechend heterogenes Datenmaterial verwendet: Die Altersspanne der Bestände beträgt 35 - 140 Jahre und die Eingriffe entsprechen B- bis E-GradDurchforstungen nach der Definition des Vereins Forstlicher Versuchsanstalten (1902). 
Als Maß zur Beschreibung der Durchforstungsart wurde mit dem von KASSIER (1993) vorgeschlagenen Stammzahl-Grundflächen-Verhältnis $N G$ ein sehr einfaches und anschauliches Maß für die Durchforstungsart verwendet. Es wird berechnet als Verhältnis der relativen Stammzahlentnahme $r N$ zur relativen Grundflächenentnahme $r G$ :

$$
N G=\frac{N_{\text {aus }} / N_{\text {ges }}}{G_{\text {aus }} / G_{\text {ges }}}=\frac{r N}{r G},
$$

wobei mit $N_{\text {aus }}$ und $G_{\text {aus }}$ die entnommene oder zu entnehmende Stammzahl bzw. Grundfläche und mit $N_{\text {ges }}$ und $G_{\text {ges }}$ die Stammzahl bzw. Grundfläche des Gesamtbestandes bezeichnet werden. Durch Umformung von Formel (1) erhält man die durchforstungsartabhängige Beziehung zwischen dem Durchmesser des Grundflächenmittelstamms des ausscheidenden $\left(D g_{\text {aus }}\right)$ und des Gesamtbestandes $\left(D g_{g e s}\right)$ :

$$
d g_{\text {aus }}=N G^{-0,5} \cdot d g_{\text {ges }}
$$

Ist $N G=1$, sind die mittleren Grundflächen des ausscheidenden Bestandes und des Gesamtbestandes identisch. In diesem Fall war der Eingriff hinsichtlich der Durchmesser unselektiv². Bei $N G<1$ wird ein geringerer Stammzahl- als Grundflächenanteil entnommen, d. h. der $D g$ des ausscheidenden Bestandes ist größer als der $D g$ des Gesamtbestandes. Es handelt sich somit - rein rechnerisch - um eine Hochdurchforstung. Umgekehrt entspricht der Eingriff bei Indexwerten $>1$ (theoretisch) einer Niederdurchforstung. Dabei ist der jeweilige Charakter der Durchforstung um so ausgeprägter, je mehr sich der $N G$-Wert von 1 unterscheidet. Allerdings zeigen die hier untersuchten Daten, daß die $N G$-Werte bei gleicher (verbal definierter) Durchforstungsart recht stark streuen und daß die Grenze zwischen Hoch- und Niederdurchforstung nicht notwendigerweise bei 1 liegt. Tatsächlich beträgt der $N G$-Wert bei den untersuchten Hochdurchforstungen im Mittel rund 1,1 und bei den Niederdurchforstungen ungefähr 1,4 (s. Abb. 2).

Als Maß für die Durchforstungsstärke kann die relative Grundflächenentnahme $r G$ oder die relative Stammzahlentnahme $r N$ verwendet werden. Auf der Basis dieser Eingangsgrößen wurde ein Durchforstungsmodell entwickelt, das die Durchmesserverteilung des ausscheidenden Bestandes mit Hilfe der Dichtefunktion der Weibullverteilung schätzt (vgl. a. Staupendahl und PuUmalainen 1999). Deren allgemeine, dreiparametrige Form lautet

$$
f(x)=\frac{c}{b} \cdot\left(\frac{x-a}{b}\right)^{c-1} \cdot e^{-\left(\frac{x-a}{b}\right)^{c}} \quad(a \leq x<\infty),
$$

\footnotetext{
${ }^{2}$ Einen Sonderfall würde die Entnahme von Bäume in den Randbereichen der Durchmesserverteilung darstellen; auch in einem solchen Fall könnte $N G$ gleich 1 sein.
} 
wobei $x$ eine stetige Zufallsvariable (hier den BHD) bezeichnet und die Parameter $a, b$ und $c$ die Lage, die Skalierung bzw. die Form der Weibullverteilung bestimmen. Ziel war die Entwicklung von Schätzfunktionen für die Parameter der Weibullfunktion, die bei gegebenem Gesamtbestand und vorgegebener Durchforstungsart eine bestmögliche Anpassung an die Durchmesserverteilung des ausscheidenden Bestandes gewährleisten. Dazu wurden zunächst die Weibull-Parameter aller 67 Durchmesserverteilungen des ausscheidenden und des Gesamtbestandes ermittelt. Bei dieser Anpassung der Weibullfunktion an die empirischen Verteilungen wurde die von GARCIA (1981) vorgeschlagene Momentmethode angewandt. Anschließend wurden mit Hilfe der Regressionsanalyse Schätzfunktionen für die Weibullparameter aufgestellt, wobei als unabhängige Einflußgrößen der $N G$-Wert sowie verschiedene Variablen des ausscheidenden und des Gesamtbestandes untersucht wurden.

Aufgrund der oft vorhandenen Korrelation der Weibullparameter untereinander wurde der $a$-Parameter der ausscheidenden Durchmesserverteilung $\left(a_{\text {aus }}\right)$ fixiert, indem für ihn der $a$ Parameter des jeweiligen Gesamtbestandes $\left(a_{g e s}\right)$ eingesetzt wurde. Die Regressionsanalyse für die Parameter $b$ und $c$ des ausscheidenden Bestandes $\left(b_{\text {aus }}, c_{\text {aus }}\right)$ ergab dann folgende linearen Schätzfunktionen (mit dem Bestimmtheitsmaß $R^{2}$ ):

$$
\begin{array}{ll}
a_{\text {aus }}=a_{\text {total }} & \\
b_{\text {aus }}=0,1230+1,0670 \cdot\left(d g_{\text {aus }}-a_{\text {aus }}\right) & R^{2}=0,99 \\
c_{\text {aus }}=8,0665+0,0554 \cdot d g_{\text {aus }}-2,2392 \cdot \ln \left(a_{\text {aus }}\right)-0.0013 \cdot N_{\text {ges }} & R^{2}=0,39
\end{array}
$$

Die Schätzfunktion für den $b$-Parameter beruht auf einer Untersuchung von GADOW (1987, S. 100), der für Plantagenbestände aus Pinus patula zwischen dem Weibull-Parameter $b$ und der Differenz aus dem arithmetischen Mitteldurchmesser $\bar{d}$ und dem Weibull-Parameter $a$ einen annähernd funktionalen Zusammenhang festgestellt hat. Die Vermutung, daß dieser Beobachtung die einfache Grundbeziehung $b=\bar{d}-a$ zugrunde liegt, wird durch die hier gefundenen Regressionskoeffizienten und das hohe Bestimmtheitsmaß bestärkt. Der Dg des ausscheidenden Bestandes wird über Formel (2) ermittelt, so daß - wie vorgesehen - die Durchforstungsart bei der Parameterschätzung berücksichtigt wird. Die Eingangsgrößen des Durchforstungsmodells sind also:

1.) Der $D g$, der $a$-Parameter und die Stammzahl des Gesamtbestandes. $D g$ und Stammzahl sollten aus den aktuellen (oder fortgeschriebenen) Forsteinrichtungsdaten bekannt sein, $a_{\text {ges }}$ ließe sich - wenn die aktuelle Durchmesserverteilung unbekannt ist - mit Hilfe des Ansatzes von GAFFREY et al. (1998) schätzen.

2.) Eine beliebige Zweierkombination der Variablen $r N, r G$ und $N G$. Der jeweils unbekannte Parameter wird dann über Gleichung (1) berechnet. Die relative Stammzahlentnahme läßt sich durch einfaches Zählen aller entnommenen Stämme unkompliziert ermitteln. Schwieriger ist die Bestimmung der relativen Grundflächenentnahme, gegebenenfalls muß hier 
eine Schätzung der Absenkung des Bestockungsgrades genügen. Alternativ könnte der $N G$-Wert, der der ausgeführten Durchforstungsart entspricht, geschätzt werden. Abb. 2 liefert hierfür entsprechende Rahmenwerte, die für die Buche auf der Auswertung des genannten Datenmaterials beruhen. Die $N G$-Werte der Fichte wurden ebenfalls auf Durchforstungsversuchsflächen der Nordwestdeutschen Forstlichen Versuchsanstalt ermittelt und man sieht sehr deutlich, daß bei gleichen Durchforstungsgraden sehr unterschiedliche Werte erreicht werden. Entsprechende Auswertungen sollen daher in Zukunft auch für die anderen Hauptbaumarten erfolgen ${ }^{3}$.
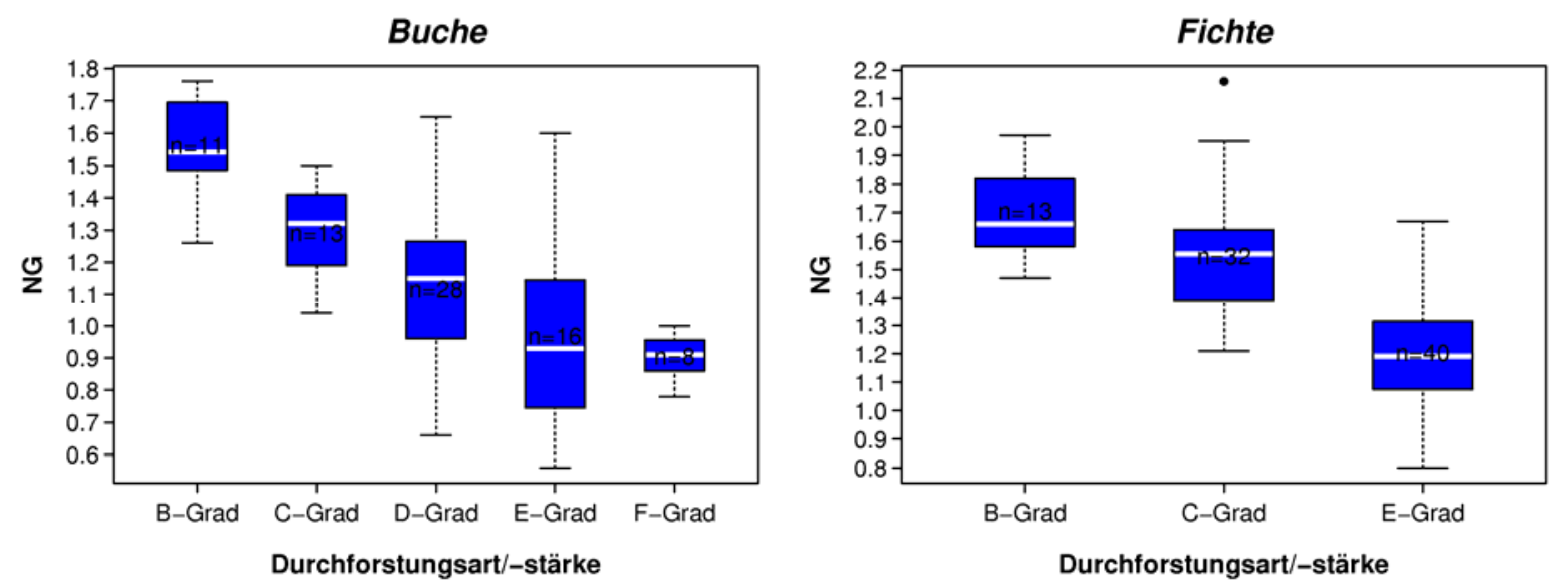

Abb. 2. Verteilung der $N G$-Werte in Abhängigkeit vom Durchforstungsgrad in 9 Buchen- und 11 FichtenDurchforstungsversuchen der Nordwestdeutschen Forstlichen Versuchsanstalt ( $n$ gibt die Anzahl der ausgewerteten Durchforstungen an).

Liegt die Durchmesserverteilung des ausscheidenden Bestandes als Schätzung vor, ergibt sich die Verteilung des verbleibenden Bestandes, indem die Durchmesserklassen-Häufigkeiten des ausscheidenden Bestandes vom Gesamtbestand subtrahiert werden. Um eine ,geglättete“ Verteilung des verbleibenden Bestandes zu erhalten, sollte als Basis für diesen Berechnungsschritt anstatt der empirischen Häufigkeiten die mit Hilfe der Momentmethode angepaßte Weibullverteilung des Gesamtbestandes verwendet werden. Ein Beispiel für eine solche Modellschätzung zeigt Abb. 3.

Zur Validierung des Durchforstungsmodells wurden die Durchmesserverteilungen von 30 Buchenreinbeständen des Staatlichen Forstamtes Paderborn (Nordrhein-Westfalen) verwendet. Im Gesamtbestand wurden jeweils mindestens 200 Bäume erfaßt, der ausscheidende Bestand war vor den Aufnahmen durch den zuständigen Revierleiter ausgezeichnet worden. Das Alter der Bestände liegt hier zwischen 50 und 140 Jahren, mit Schwerpunkt bei den mittleren Altersklassen. In allen Durchmesserverteilungen wurde nur der Oberstand berücksich-

\footnotetext{
3 Zur Erklärung der z. T. starken Streuung der $N G$-Werte innerhalb eines Durchforstungsgrades s. STAUPENDAHL (1999) und STAUPENDAHL und PUUMALAINEN (1999).
} 
tigt. Mit Hilfe des Durchforstungsmodells wurden nun die ausscheidenden und verbleibenden Durchmesserverteilungen geschätzt und als Kriterien für die Schätzgüte die maximale absolute Differenz KS zwischen der empirischen und geschätzten Verteilungsfunktion (Kolmogorov-Smirnov-Test), die relative Diskrepanz $r D$ (vgl. GREGORIUS 1974) ${ }^{4}$, und der relative Fehler des $D g$ und der Grundfläche berechnet. Als Eingangsgrößen für die Modellschätzungen wurden die wahren Werte aus den Vollkluppungen verwendet, da hier allein der Modellfehler untersucht werden sollte.
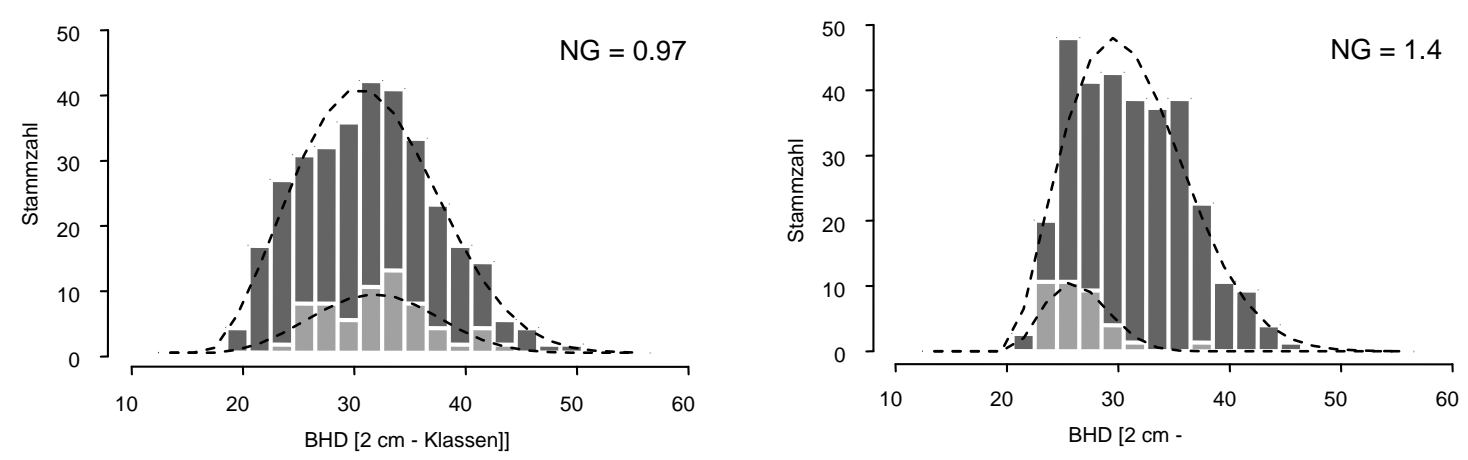

Abb. 3. Wahre und geschätzte Durchmesserverteilungen von zwei Beständen des Forstamtes Paderborn mit unterschiedlicher Durchforstungsart. Wie auch die $N G$-Werte anzeigen, wurde der linke Bestand hoch-, der rechte niederdurchforstet. Die Säulen repräsentieren die empirischen Häufigkeiten, (dunkelgrau: verbleibender, hellgrau: ausscheidender Bestand), die geschätzten Verteilungen werden durch die gestrichelten Linien angedeutet. Als Eingangsgrößen für das Schätzmodell wurden die wahren Werte aus Vollkluppungen verwendet.

Die Ergebnisse der Validierung zeigen, daß sowohl die Mittelwerte, als auch die Streuung der Prüfgrößen für den verbleibenden Bestand deutlich geringer sind als für den ausscheidenden Bestand (vgl. Abb. IV-2). Vergleicht man die KS-Werte mit den kritischen Grenzen des Kolmogorov-Smirnov-Tests für zwei Stichproben bei einer Irrtumswahrscheinlichkeit von $5 \%$, entstammen allerdings in allen Beständen die geschätzte und die empirische Verteilung der gleichen Grundgesamtheit. Die Fehler des $D g$ und der Grundfläche deuten im ausscheidenden Bestand eine leicht unterschätzenden Tendenz des Verfahrens an, sind aber selbst dort sehr gering $(>-1 \%$ bzw. $>-3 \%)$ und damit in der praktischen Anwendung zu vernachlässigen. Allerdings ist daran zu denken, daß bei unzuverlässigen Daten über den Gesamtbestand und/oder die Entnahmequoten von Grundfläche und Stammzahlmuß mit höheren Fehlern zu rechnen ist.

Obwohl das Verfahren primär für Buchenreinbestände mit unimodaler Durchmesserverteilung entwickelt wurde, scheint eine Anwendung auf andere Baumarten vertretbar zu sein,

\footnotetext{
${ }^{4}$ Mit dieser Prüfgröße wird der relative Anteil angegeben, den man zwischen den Klassen austauschen müßte, um aus der geschätzten Verteilung die empirische Verteilung entstehen zu lassen. 1-rD ist entsprechend der Anteil, der beiden Verteilungen gemeinsam ist. Der Wert 1 bedeutet, da $\beta$ die beiden Verteilungen keine gemeinsamen Klassen besitzen, während es sich bei $r D=0$ um absolut identische Verteilungen handelt.
} 
sollte aber in einer weiteren Arbeit überprüft werden. Der Einsatz des Modells in (mehrschichtigen) Mischbeständen ist ebenfalls denkbar, wenn jede Baumart, getrennt nach Bestandesschichten, als Reinbestand betrachtet wird. 


\section{Erfassung des verbleibenden Bestandes}

\subsection{Auswahl des Inventurverfahrens}

Will man das Kosten-Nutzen-Verhältnis der Waldinventur optimieren (Abb. 4), sollte nicht nur das Inventurintervall, sondern auch die Art der Bestandeserfassung an den tatsächlichen Informationsbedarf angepaßt werden. So erscheint z. B. der Aufwand einer Stichprobenerhebung in jungen, gleichaltrigen Reinbeständen - gemessen am Wert der zusätzlich gewonnenen Informationen - nicht gerechtfertigt. Unter betriebswirtschaftlichen Gesichtspunkten steigt der Informationsbedarf normalerweise mit steigendem Stammholzanteil. Neben Beständen, die aus anderen Gründen von besonderer Bedeutung sind (Sonderbiotope, Flächen des Vertragsnaturschutzes, etc.), sollten deshalb je nach Baumart nur Bestände mit einem mittleren Durchmesser von mind. 25 - 30 cm mit einer Stichprobeninventur erfaßt werden (s. Kap. 4.3). In allen anderen Fällen ist der Waldbegang mit einer schichten- und baumartenspezifischen Schätzung von Grundfläche pro ha, Mittelhöhe und Mitteldurchmesser, oder sogar nur eine summarische Erfassung und Planung auf der Basis von Bestandestypen ${ }^{5}$ eine angemessene Alternative (vgl. z. B. SPELLMANN und AKÇA 1983). Die auch in diesen Wirtschaftseinheiten interessierende Durchmesserverteilung kann mit der von GAFFREY et al. (1998) vorgestellten Methode, die die Normal- bzw. Weibullverteilung nutzt und deren Parameter anhand von Bestandesmittelwerten vorhersagt, relativ genau geschätzt werden (vgl. a. NAGEL und BIGING 1995).

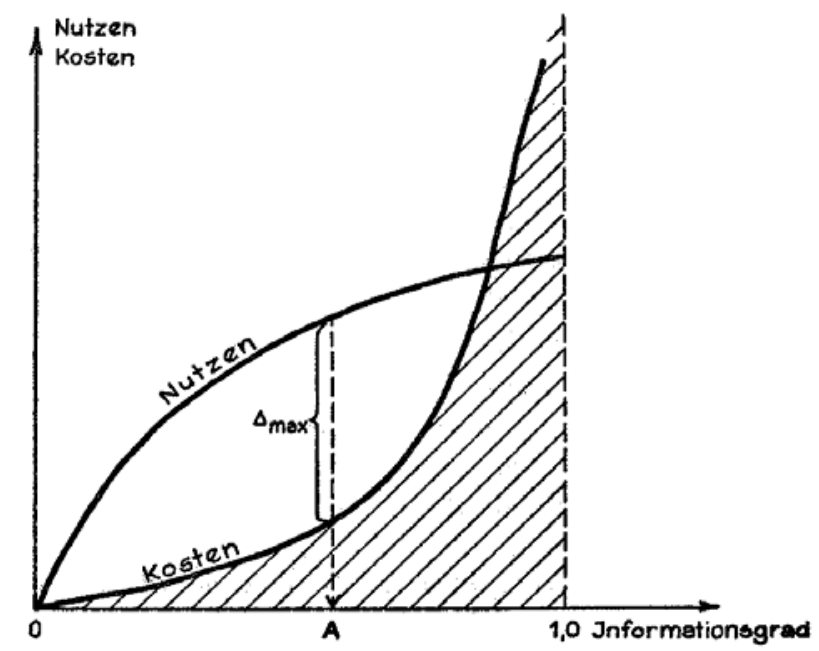

Abb. 4. Bestimmung des optimalen Informationsgrades $(A)$ mit Hilfe der Nutzen- und Kostenfunktion (SPEIDEL 1972, S. 31).

\footnotetext{
${ }^{5}$ Als Bestandestypen werden hier nach Bestandesstruktur, Baumartenzusammensetzung, Bestandesqualität und natürlicher Altersstufe zusammengefaßte Straten bezeichnet.
} 
Die Entscheidung, welches Verfahren gewählt wird, liegt jedoch vollkommen im Ermessen des Forstbetriebes und sollte sich allein an dessen Zielen sowie am Verhältnis von Kosten und Nutzen der Informationen orientieren. Abb. 4 stellt hierfür ein Kalkül zur Verfügung. Demnach ist derjenige Informationsgrad optimal, der den größten Nutzen(Ertrags)-Überschuß aus den vorhandenen Informationen über deren Beschaffungskosten gewährleistet. Die besondere Schwierigkeit besteht allerdings darin, den Nutzen zu quantifizieren.

Hierfür müßten die Auswirkungen zusätzlicher Informationen auf das Betriebsergebnis abgeschätzt werden, was angesichts der Komplexität der Zusammenhänge und v. a. der Länge der Produktionszeiträume nur sehr ungenau gelingen kann. Zur Klärung dieser Fragen wären daher weitere Untersuchungen dringend erforderlich. Abb. 5 zeigt einen Vorschlag für einen Kriterienkatalog, der bei der Entscheidung über Zeitpunkt und Art der Inventur als Orientierungshilfe dienen könnte.

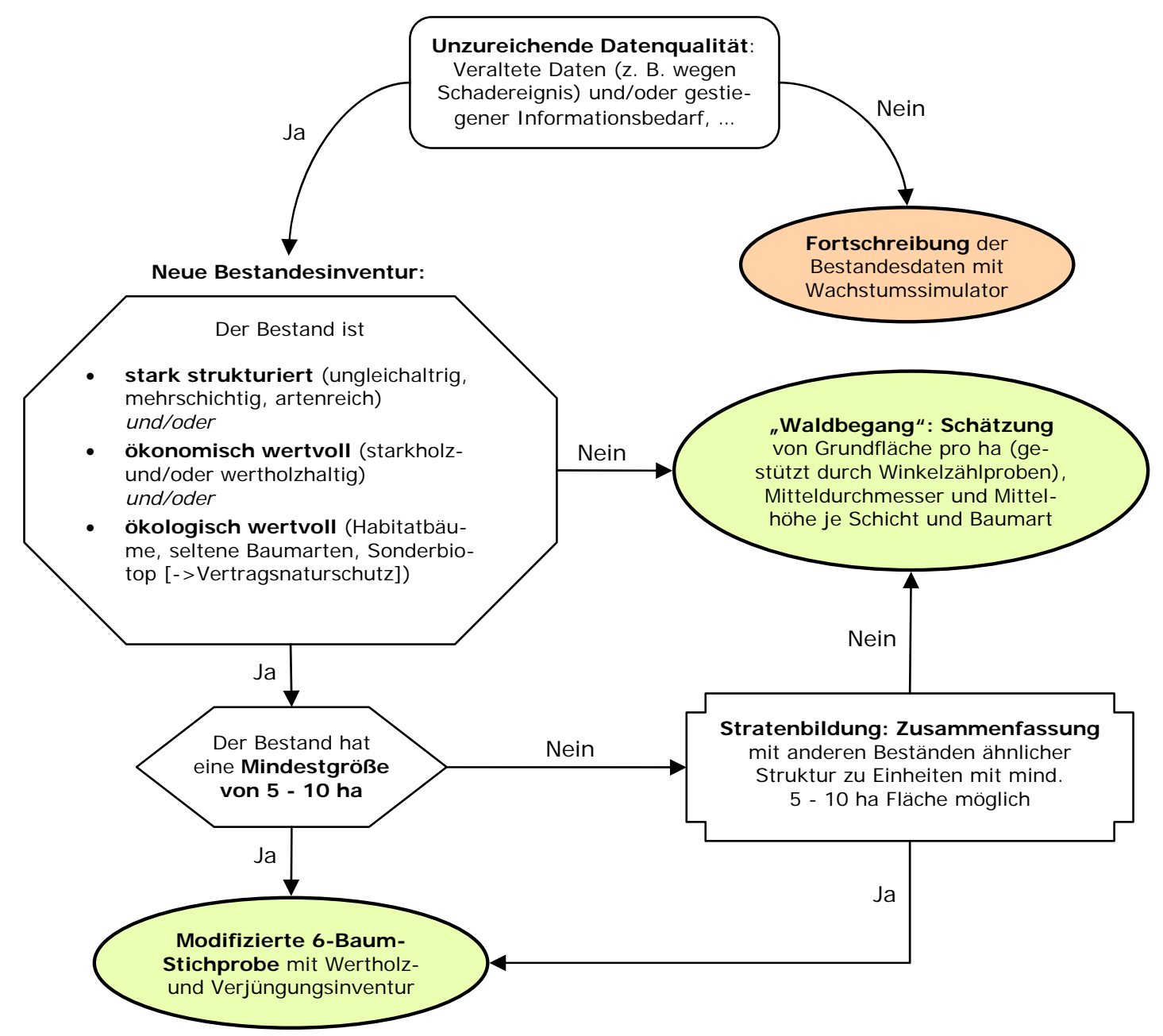

Abb. 5. Entscheidungsbaum als Hilfestellung zur Beantwortung der Frage, ob und wie die Daten eines Bestandes (verbleibender Bestand) neu erhoben werden sollen.

U. a. sollte berücksichtigt werden, daß eine Stichprobeninventur aufgrund der relativ hohen Kosten i.d.R. nur in Beständen ab einer Größe von 5 - 10 ha wirtschaftlich vertretbar ist (vgl. 
NICKE 2005). Erfüllt ein einzelner Bestand diese Voraussetzung nicht, sollte geprüft werden, $\mathrm{ob}$ es innerhalb des Forstbetriebes ähnliche strukturierte Bestände gibt, die zusammengefaßt eine ausreichende Größe erreichen. Unabhängig vom gewählten Inventurverfahren gilt jedoch immer das „Eingriffsprinzip“, d. h. das in Kap. 2 vorgestellte Verfahren der getrennten (und vollständigen) Erfassung des ausscheidenden Bestandes, die zeitliche Orientierung der Inventur am Eingriff und die Fortschreibung der Bestandesdaten.

\subsection{Inventurintervall}

Entsprechend dem in Abb. 1 dargestellten Schema könnte die Inventur des verbleibenden Bestandes bereits nach jedem nächsten Eingriff wiederholt werden. Dies würde jedoch bei den üblichen Durchforstungsintervallen zu nicht vertretbaren Inventurkosten führen. Darüber hinaus sollte bei der Wahl des Inventurintervalls auch darauf geachtet werden, daß die Veränderung der hauptsächlich interessierenden Variablen mit einem angemessenen relativen Fehler geschätzt werden. Als wichtigste Variablen werden hier die Mittelhöhe, der Mitteldurchmesser und die Bestandesgrundfläche betrachtet. Letztere steht in einem linearen Zusammenhang zum Bestandeswert und ist - im Gegensatz zur berechneten Variable Holzvorrat - direkt meßbar. Nach den Regeln der Fehlerfortpflanzung ist bei temporären Stichproben der absolute Fehler der Veränderung eines Merkmals zwischen zwei Inventuren gleich der Quadratwurzel aus der Summe der Varianzen der Zustandsschätzungen (АKÇA 2001, S. 30 f.).

Eine Folgeinventur sollte also nicht stattfinden, wenn sich die interessierenden Variablen aufgrund geringen Zuwachses und/oder kompensatorisch wirkender Nutzungen kaum verändert haben, da in einem solchen Fall bei vertretbarem Stichprobenumfang die Schätzung der Veränderung im Stichprobenfehler ,untergeht“ und damit vollkommen unklar ist, ob die festgestellte Zustandsänderung der Wirklichkeit entspricht oder durch Zufallseffekte der Stichprobenziehung nur suggeriert wird. Wenn der angestrebte relative Stichprobenfehler (Standardfehler) der Zustandsgrößen aus wirtschaftlichen Gründen auf einen bestimmten Wert festgelegt ist, wird der Fehler der Schätzung der Zustandsänderung also erst dann ein vertretbares Maß erreichen, wenn die Änderung ausreichend groß ist. Von Schadereignissen abgesehen wird dies je nach Wuchsdynamik eines Bestandes nach unterschiedlichen Zeiträumen der Fall sein.

Wenn man die wahren Werte der zu schätzenden Variablen als Bezugsgrößen verwendet, lautet die Formel zur Berechnung des relativen Standardfehlers der Zustandsänderung:

$$
s_{\overline{\Delta X}} \%=\frac{\sqrt{s_{\bar{X}_{1}}{ }^{2}+s_{\bar{X}_{2}}{ }^{2}}}{\bar{X}_{2}-\bar{X}_{1}}=\frac{s_{\bar{X}} \% \cdot \sqrt{\bar{X}_{1}^{2}+\bar{X}_{2}^{2}}}{\bar{X}_{2}-\bar{X}_{1}},
$$


mit $\bar{X}_{1}, \bar{X}_{2}=$ Schätzwerte der Variablen $X$ zum Zeitpunkt 1 (Erstinventur) und 2 (Folgeinventur)

$s_{\bar{X}_{1}}, s_{\bar{X}_{2}}=$ absolute Standardfehler der Variablen $X$ zu den Zeitpunkten 1 und 2

$s_{\bar{X}} \%=$ konstanter relativer Standardfehler der Variablen $X=s_{\bar{X}_{1}} / \bar{X}_{1}=s_{\bar{X}_{2}} / \bar{X}_{2}$

$\overline{\Delta X}=\bar{X}_{2}-\bar{X}_{1}$

Aus (5) folgt, daß die nächste Inventur um so später erfolgen sollte, je größer die Werte der Zielvariablen $\left(\bar{X}_{1}^{2}+\bar{X}_{2}^{2}\right)$ und deren relative Standardfehler $\left(s_{\bar{X}} \%\right)$ sind und je weniger sich die Variablenwerte ändern $\left(\bar{X}_{2}-\bar{X}_{1}\right)$. Abb. 6 verdeutlicht diese Zusammenhänge anhand von exemplarischen Daten aus der SCHOBER'schen Ertragstafel für Buche, I. Ertragsklasse, mäßige Durchforstung (SCHOBER 1995). Sie zeigt den mit Formel (5) berechneten Standardfehler der Zuwachsraten der Zielgrößen für Folgeinventuren in unterschiedlichen Zeitabständen zu zwei Erstinventuren im Bestandesalter 40 und 65. Bei der Kalkulation wurde ein Standardfehler der Zustandsschätzung von $10 \%$ unterstellt. Stichprobensimulationen mit dem in Kap. 4.3 beschriebenen Verfahren haben ergeben, daß dieser Fehlerwert auch in strukturreichen Beständen mit ca. 20 - 40 Probekreisen je Bestand eingehalten werden kann, so daß es sich hierbei um eine realistische Vorgabe handelt.

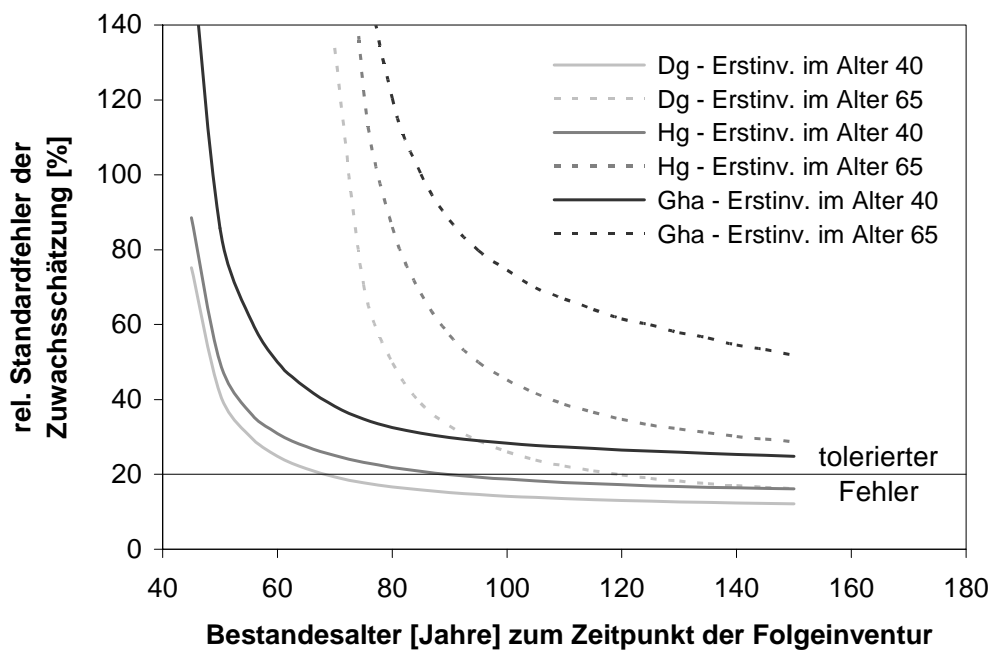

Abb. 6. Theoretischer Standardfehler der Schätzung des Zuwachses von Mitteldurchmesser $(D g)$, Mittelhöhe $(H g)$ und Grundfläche pro ha $(G h a)$ in Abhängigkeit vom Inventurintervall, dargestellt für zwei Erstinventuren im Bestandesalter 40 und 65, bei einem angenommenen Standardfehler der Zustandsschätzungen von $10 \%$. Die Fehlerberechnung beruht auf den Tafelwerten für den verbleibenden Bestand der SCHOBER'schen Ertragstafel für Buche (I. Ertragsklasse, mäßige Durchforstung).

Wird das Doppelte des Fehlers der Zustandsschätzung, also $20 \%$, als ein tolerabler Fehler der Zuwachsschätzung betrachtet, so wird dieser Wert bei der Grundflächenzuwachsschätzung innerhalb des Produktionszeitraumes weder für die Erstaufnahme im Alter 40 noch für die Aufnahme im Alter 65 jemals erreicht. Bei der Höhenzuwachsschätzung wird er ab einem Inventurintervall von 50 Jahren unterschritten, wenn der Bestand im Alter 40 zum ersten Mal aufgenommen wurde, bei einer Erstinventur im Alter 65 liegen die Fehlerwerte ebenfalls im- 
mer über $20 \%$. Lediglich bei der Schätzung des mittleren Durchmesserzuwachses nähern sich die Inventurintervalle mit knapp 30 Jahren (Erstinventur im Alter 40) bzw. ca. 55 Jahren (Erstinventur im Alter 65) allmählich dem in der Forsteinrichtung üblichen Inventurabstand von 10 Jahren an. Die Sicherheit, daß der wahre Zuwachs dann tatsächlich um nicht mehr als $20 \%$ vom Schätzwert abweicht, beträgt allerdings nur ca. $68 \%$, da kein erweitertes Vertrauensintervall berechnet wurde.

Diese Inventurintervalle ließen sich drastisch verkürzen, wenn der geforderte Fehler der Zustandsschätzung reduziert würde. Allerdings würde damit der notwendige Stichprobenumfang überproportional ansteigen, was aus wirtschaftlichen Gründen nicht akzeptabel ist. Diese Zusammenhänge, die in ähnlicher Größenordnung auch für die anderen Hauptbaumarten gelten, zeigen, daß bei temporären Stichproben auf Bestandesebene und unter den in Deutschland üblichen Durchforstungsintensitäten und Wuchsbedingungen eine Wiederholungsinventur nach weniger als 20 Jahren eigentlich nutzlos ist.

Mit Rücksicht auf die Fortschreibung sollte das Inventurintervall aber auch nicht wesentlich länger sein, da sich die Zuverlässigkeit der Wachstumsprognose mit länger werdendem Fortschreibungszeitraum deutlich verschlechtert. Zusammenfassend kann also ein Inventurintervall zwischen 15 und 25 Jahren empfohlen werden. Diese langen Inventurintervalle und das damit verbundene Einsparpotential im Bereich der Datenerhebung werden jedoch erst durch die genaue Erfassung der Nutzungen und deren Einarbeitung in die mit Wuchsmodellen fortgeschriebenen Bestandesdaten möglich. Die Lösung von starren Inventurintervallen erlaubt es, den Inventuraufwand an die individuellen Gegebenheiten anzupassen, wodurch überflüssige Inventurarbeiten vermieden werden, die keinen oder nur einen sehr geringen Informationsgewinn bringen. Auf der anderen Seite kann genau dort eine höhere Genauigkeit erzielt werden, wo sie notwendig und erwünscht ist.

Natürlich stellt sich in diesem Zusammenhang auch die Frage nach permanenten Stichproben, deren Zuwachsschätzung bei gleichem Stichprobenumfang i.d.R. erheblich genauer ist, wenngleich die Kosten je Probefläche um einiges höher sind, da jede von ihnen genau eingemessen und dauerhaft markiert werden muß. Diese zusätzlichen Kosten können jedoch durch einen geringeren notwendigen Stichprobenumfang mehr als kompensiert werden. $\mathrm{Ob}$ dies der Fall ist, hängt entscheidend davon ab, wie stark die Probekreiswerte der Erst- und Folgeinventur korreliert sind (vgl. AKÇA 2001, S. 132). Bei Wachstumsgrößen wird diese Korrelation um so deutlicher sein, je kleiner das Inventurintervall ist und je weniger die Bestandesstruktur innerhalb dieses Zeitraumes durch Nutzungen oder Störungen verändert wurde. Aus Kostengründen verbietet sich allerdings ein kurzes Inventurintervall und in intensiv bewirtschafteten Wäldern, also gerade dort, wo Eingriffsinventuren interessant sind, werden die nutzungsbedingten Veränderungen nicht unerheblich sein. Aus diesen Gründen wird im Rahmen von Eingriffsinventuren, von wenigen Ausnahmen abgesehen, vermutlich die temporäre Stichprobe effizienter sein. 


\subsection{Die modifizierte 6-Baum-Stichprobe}

Die auf der Messung von Punkt-Baum-Abständen beruhende 6-Baum-Stichprobe (PRODAN 1968) ist als einfach anzuwendendes und kostengünstiges Inventurverfahren bekannt, das - im Gegensatz zu Inventuren mit festen bzw. konzentrischen Probekreisen - keinen Spezialisten, sondern nur den eingeübten Mitarbeiter der Forsteinrichtung erfordert (HIRNER 1978, S. 2). Insbesondere kann es als Ein-Personen-Verfahren angewandt werden, was die Inventurkosten erheblich reduziert (vgl. NiCKE 2005). Für die Anwendung im Rahmen von Eingriffsinventuren ist es daher besonders geeignet. Die Stichprobeneinheit ist bei der 6-BaumStichprobe durch die vom Stichprobenpunkt $i$ aus gesehen sechs nächsten Bäume definiert, mit $i=1 . . n$. An jedem der systematisch (AKÇA 2001, S. 41 f.) über die Bestandesfläche verteilten $n$ Stichprobenpunkte werden die Art, der Brusthöhendurchmesser und gegebenenfalls weitere Attribute der Probebäume erhoben. Der Abstand vom Stichprobenpunkt zur Stammachse des sechstnächsten Baumes liefert den Radius $r_{i}$ des (ideellen) Probekreises (Abb. 7), auf dessen Basis die Schätzung der flächenbezogenen Zielvariablen erfolgt.

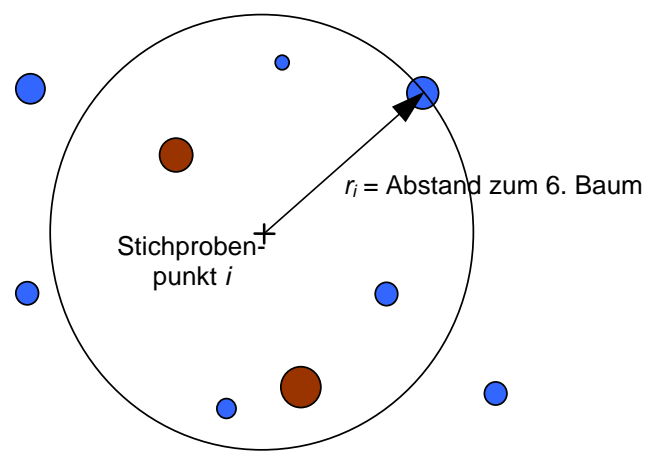

Abb. 7. Schema eines Probekreises der 6-Baum-Stichprobe. Der Probekreisradius entspricht dem Abstand vom Stichprobenpunkt zur Stammachse des sechstnächsten Baumes, die Probekreisfläche ist folglich $a_{i}=\pi \cdot r_{i}^{2}$.

Allerdings ist bekannt, daß die Schätzung der Stammzahl und Grundfläche pro ha bei nichtzufälligen Baumverteilungsmustern verzerrte Ergebnisse liefert. JONSSON et al. (1992) haben gezeigt, daß das Vorzeichen des systematischen Fehlers (Bias) bei regelmäßiger Verteilung negativ und bei geklumpter Verteilung positiv ist, und daß der Fehlerbetrag mit zunehmender Abweichung von der Zufallsverteilung zunimmt. Aus diesem Grund wurde mit dem punktbezogenen Winkelmaß $W_{P}$ ein auf dem Ansatz von GADOW et al. (1998) beruhender Aggregationsindex entwickelt, der in Kombination mit der 6-Baum-Stichprobe schnell und einfach zu bestimmen ist und eine enge Korrelation zur Richtung und zum Ausmaß der Verzerrung zeigt. Im folgenden sollen zunächst das punktbezogene Winkelmaß und seine statistischen Eigenschaften und anschließend ein Regressionsmodell vorgestellt werden, das den Zusammenhang von Stammzahlbias und Winkelmaß beschreibt und damit eine ausreichend genaue Korrektur des Bias ermöglicht. 


\subsubsection{Das punktbezogene Winkelmaß}

Neben dem hier verfolgten Zweck der Biaskorrektur stellt die quantitative Beschreibung der räumlichen Struktur von Wäldern natürlich auch einen eigenständigen Wert dar. Die intensiv geführte Diskussion über eine nachhaltige Bewirtschaftung der Wälder und deren Zertifizierung hat gezeigt, daß ein großer Bedarf an Indikatoren besteht, mit denen sich die Biodiversität von Wäldern objektiv messen und in ihrer Veränderung beobachten läßt (vgl. MCPFE 2003). In diesem Zusammenhang spielen Indikatoren zur Beschreibung von Waldstrukturen eine wichtige Rolle (KÖHL und ZINGG 1995; SZARO und JOHNSTON 1996; VANCLAY 1998), da die Eignung eines Waldes als Lebensraum für andere Pflanzen und Tiere und als Erholungsraum für den Menschen ganz wesentlich von seiner vertikalen und horizontalen Struktur abhängt (OtTo 1994, S. 180 ff.; SPELlmanN 1995; Kimmins 1997, S. 352 ff.).

Auch auf den Zuwachs übt die räumliche Bestandesstruktur einen erheblichen Einfluß aus, wie Untersuchungen von PRETZSCH (1995) deutlich gezeigt haben. Sollen abstandsabhängige Einzelbaummodelle auch dann zum Einsatz kommen, wenn die Baumkoordinaten unbekannt sind (und dies ist die Regel), hängt die Genauigkeit der Prognose ganz wesentlich von der Verwendung geeigneter Strukturindizes ab (PRETZSCH 1997; POMMERENING 1998). Diese sollten sich im Rahmen von Inventuren einfach und kostengünstig erheben lassen und in einem zweiten Schritt die realistische Reproduktion der Bestandesstrukturen erlauben (vgl. Lewandowski und GAdOw 1997; Pretzsch 1997; Pommerening 2006).

Zur numerischen Beschreibung von Waldstrukturen wurden in der forstlichen Forschung zahlreiche Strukturindizes entwickelt oder aus anderen Wissenschaftsdisziplinen übernommen (vgl. UPTON und FINGLETON 1989, 1990; BIBER 1997; GLEICHMAR und GEROLD 1998; SMALTSCHINSKI 1998; GADOW 1999). Das punktbezogene Winkelmaß basiert auf dem von GADOW et al. (1998) vorgestellten Ansatz der Klassierung der Winkel zwischen den $k$ nächsten Nachbarn des sogenannten Nullbaumes, d. h. des Baumes mit dem kürzesten Abstand zum Stichprobenpunkt $i$. Wie der Name bereits sagt, ist die Bezugsbasis beim punktbezogenen Winkelmaß statt des Nullbaumes der Stichprobenpunkt selbst: Für jeden Baum $j$ der Stichprobeneinheit $i$ wird der vom Stichprobenpunkt ausgehende Winkel $\alpha_{i j}$ zum jeweiligen Nachbarbaum bestimmt (mit $j=1 . . k$ ), wobei als Nachbar der in einer vorher festgelegten Drehrichtung als nächstes folgende Baum betrachtet wird. Einen Sonderfall stellt die Situation dar, in der der Winkel zwischen zwei benachbarten Bäumen mehr als $180^{\circ}$ beträgt. Zwischen diesen Bäumen wird dann in umgekehrter Richtung gemessen, $\alpha_{i j}$ ist also immer $\leq 180^{\circ}$ (s. Abb. 8). Jeder dieser Winkel wird mit dem kritischen Winkel $\alpha_{c}$ verglichen ${ }^{6}$. Das punktbezogene Winkelmaß ist dann definiert als der Anteil der Winkel $\alpha_{i j}$, die kleiner sind als der kritische Winkel $\alpha_{c}$ :

\footnotetext{
${ }^{6}$ Der konkrete Wert von $\alpha_{c}$ wird, wie ab Seite 25 gezeigt wird, in Abhängigkeit von der Mächtigkeit eines Winkelmaß-basierten Tests auf vollständig zufällige räumliche Verteilung bestimmt.
} 


$$
W_{P, i}=\frac{1}{k} \cdot \sum_{j=1}^{k} z_{i j}, \text { mit } z_{j}=\left\{\begin{array}{l}
1 \text { falls } \alpha_{i j}<\alpha_{c} \\
0 \text { sonst }
\end{array}, \alpha_{c} \leq 360 / k \text { und } 0 \leq W_{P, i} \leq 1 .\right.
$$

Aus (6) folgt, daß $W_{P, i}$ nur $k+1$ verschiedene Werte annehmen kann. $W_{P, i}=0$ deutet auf eine stark ausgeprägte Regelmäßigkeit hin, während bei $W_{P, i}=1$ eine deutliche Klumpung zu erwarten ist. Wie groß $k$ gewählt wird, hängt vom jeweiligen Kontext ab, in dem das Winkelmaß erhoben wird. Grundsätzlich gilt, daß eine zuverlässige Identifizierung des $k$-ten Baumes allein durch okulare Einschätzung mit wachsendem $k$ immer schwieriger wird und damit immer öfter zeitaufwendige Abstandsmessungen nötig werden. Im Sinne eines optimalen Verhältnisses von Aufwand und Informationsdichte haben sich daher - wenn es allein um die Erfassung der räumlichen Verteilung geht - drei Bäume $(k=3)$ als am besten geeignet erwiesen. Im Rahmen der 6-Baum-Stichprobe sollten jedoch sechs Bäume verwendet werden (Abb. 8), da der sechste Baum hier ja schon ermittelt ist und jedes davon abweichende $k$ in Zweifelsfällen nur zusätzliche und unnötige Arbeit bedeuten würde.
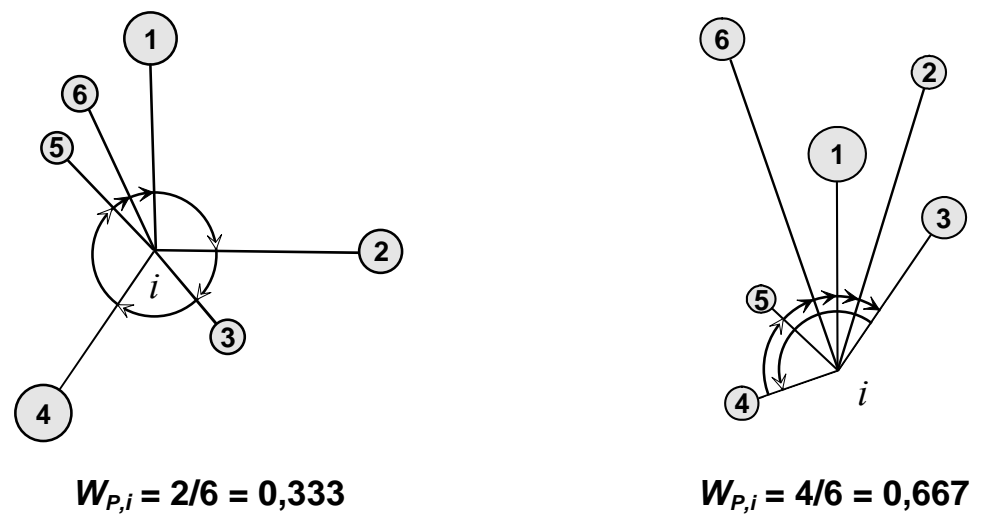

Abb. 8. Zwei mögliche Werte des punktbezogenen Winkelmaßes $W_{P}$ mit $k=6$ und kritischem Winkel $\alpha_{c}=35^{\circ}$ (s. hierzu auch S. 25 ff.). Die Bäume sind nach ihrem Azimut aufsteigend numeriert, der Nachbar eines Baumes ist also durch die nächst größere Baumnummer gekennzeichnet. Ist die Pfeilspitze des zugehörigen Winkelbogens schwarz, ist $\alpha_{i j}$ kleiner als $\alpha_{c}$, andernfalls größer. Die Abbildung rechts macht deutlich, daß zwischen zwei benachbarten Bäumen immer der kleinere der beiden möglichen Winkel gemessen wird!

Da für die Bestimmung des Winkelmaßes kein exakter Winkel benötigt wird, sondern nur geprüft werden muß, ob ein Winkel kleiner oder größer als ein bestimmter Grenzwert ist, läßt sich die Bestimmung des $W_{P, i}$-Wertes deutlich beschleunigen, wenn man das am Institut für Waldinventur und Waldwachstum der Universität Göttingen entwickelte „Winkelvisier“ verwendet: Dieses einfache Meßinstrument besteht aus zwei auf einem Stativ strahlenförmig befestigten, schmalen Schienen, auf denen eine Visierlinie angebracht ist und deren Winkel zueinander auf den kritischen Winkel eingestellt wird. Das Stativ wird am Stichprobenpunkt positioniert und mit einer der beiden Schienen wird die Stammachse des $j$-ten Baums anvisiert. Blickt man nun über die andere Schiene, läßt sich schnell und zuverlässig bestimmen, 
ob der Nachbarbaum inner- oder außerhalb des kritischen Winkels liegt. Mit Hilfe dieses Instruments läßt sich das Winkelmaß i.d.R. in weniger als 1 min. bestimmen.

Ausgehend von einer systematischen Stichprobe des Umfangs $n$ ist

$$
\hat{W}_{P}=\frac{1}{n} \cdot \sum_{i=1}^{n} W_{P, i}
$$

ein erwartungstreuer Schätzer für den $W_{P}$-Wert des Bestandes. In Ermangelung eines Schätzers für den Standardfehler (Stichprobenfehler) im Fall einer systematischen Stichprobe wird in der Regel der entsprechende Schätzer für die Zufallsstichprobe verwendet (AKÇA 2001, S. 114 ff.). Stichprobensimulationen in drei künstlich erzeugten Baumpopulationen mit stark geklumpter, zufälliger und sehr regelmäßiger Verteilung ergaben, daß die darauf beruhenden Schätzungen bei Stichprobenumfängen $>5 \mathrm{sehr}$ nah bei den wahren Standardfehlern liegen, und zwar auch bei nicht-zufälliger Verteilung der Bäume. Bei darunterliegenden Stichprobenumfängen (die allerdings praktisch kaum von Bedeutung sind), ergab sich eine leichte Unterschätzung (vgl. Abb. III-9).

Wie bei allen Aggregationsindizes besteht auch beim Winkelmaß am Bestandesrand generell eine höhere Tendenz zur Klumpung, da jenseits dieser Grenze keine Bäume ausgewählt werden sollen oder können (Randeffekt). Liegt der Stichprobenpunkt z. B. direkt neben einer gerade verlaufenden Bestandesgrenze, liegt das theoretische Minimum des $W_{P, i}$-Wertes (bei $k=6$ ) selbst bei absolut regelmäßiger Verteilung der Randbäume bei 0,17 , da der Suchwinkel für die sechs nächsten Bäume auf $180^{\circ}$ beschränkt ist. Deshalb sollten bei der Berechnung des Winkelmaßes gemäß Schätzer (7) nur diejenigen Probekreise berücksichtigt werden, die den Bestandesrand nicht schneiden. Dadurch ist jegliche Beeinflussung des $W_{P}$-Wertes durch Randeffekte ausgeschlossen.

Die Bestimmung des wahren Wertes des punktbezogenen Winkelmaßes $W_{P}$ ist insofern problematisch, als er exakt nur durch Integration aller $W_{P, i}$ über jeden Punkt der untersuchten Fläche ermittelt werden kann und es eine unendliche Anzahl von Punkten gibt. Allerdings kann der ,wahre“ $W_{P}$-Wert approximiert werden, indem man die Fläche mit Hilfe eines Gitternetzes in eine endliche Anzahl von Pixeln zerlegt, für jedes Pixel den $W_{P, i}$-Wert bestimmt und aus diesen den Mittelwert berechnet. Natürlich ist diese Annäherung umso genauer, je feiner die Auflösung, d. h. je kleiner die Pixel sind. Da dies aber gleichzeitig den Rechenaufwand immer mehr erhöht, gilt es einen Kompromiß zu finden. Eine Analyse der Schwankungen der $W_{P}$-Werte bei unterschiedlichen Pixelgrößen ergab, daß mit einer Pixelgröße von $5 \%$ der mittleren Baumstandfläche (= Bestandesfläche/Stammzahl) ausreichend genaue und zuverlässige Schätzungen des wahren Wertes erreicht werden.

Die Häufigkeitsverteilung der $W_{P, i}$ bei einer vollständig zufälligen räumlichen Verteilung der Bäume (complete spatial randomness = CSR) kann analytisch oder durch Monte Carlo Simulationen bestimmt werden (vgl. a. Abb. III-8b). Auf dieser Grundlage läßt sich an- 
hand eines $C h i^{2}$-Anpassungstest die Nullhypothese (CSR) gegen Alternativhypothesen testen, mit

$$
T=\sum_{i=1}^{k+1} \frac{\left(O_{i}-E_{i}\right)^{2}}{E_{i}},
$$

wobei $O_{i}$ die beobachteten Häufigkeiten und $E_{i}$ die unter der Nullhypothese zu erwartenden Häufigkeiten bezeichnet. Für große Stichprobenumfänge ist $T$ annähernd $\chi^{2}$-verteilt, mit $k$ Freiheitsgraden. Allerdings ist diese Approximation ungenau für kleine Stichprobenumfänge, besonders dann, wenn eines der $E_{i}$ kleiner ist als fünf. Da bei den hier relevanten Stichprobenumfängen die kleinste zu erwartende Häufigkeit viel kleiner ist als fünf, war es notwendig, eine bessere Annäherung an die Verteilung von $T$ zu finden, um genaue kritische Werte für den Test zu erhalten. Hierfür wurden per Simulation eine Million unabhängige Realisierungen von $T$ unter der Nullhypothese erzeugt. Wie erwartet unterschied sich die resultierende Verteilung deutlich von der $\chi^{2}$-Verteilung, näherte sich dieser mit wachsendem Stichprobenumfang jedoch immer mehr an.

Wie sich herausstellte, hängt die Verteilung von $T$ bei CSR - und damit die Teststärke vom kritischen Winkel $\alpha_{c}$ ab. Und zugleich zeigte sich, daß derjenige Wert von $\alpha_{c}$, der die Mächtigkeit des Tests gegen die Alternativhypothese „Die Verteilung ist geklumpter als bei CSR" maximiert, sich deutlich von dem $\alpha_{c}$ unterscheidet, das beim Test gegen die Alternativhypothese „Die Verteilung ist regelmäßiger als bei CSR“ die maximale Teststärke liefert. Somit müßte, um die höchstmögliche Teststärke zu erreichen, vor der Inventur bestimmt werden, gegen welche Alternativhypothese getestet werden soll. In der Praxis dürfte aber meistens im vornherein unklar sein, ob das Baumverteilungsmuster eher regelmäßig oder geklumpt ist. In diesen Fällen sollte ein $\alpha_{c}$ benutzt werden, das zwischen den optimalen Werten für die oben genannten Alternativhypothesen liegt und einen bestmöglichen Kompromiß darstellt.

Um die zwei optimalen kritischen Winkel zu ermitteln, wurden Monte Carlo Simulationen mit regelmäßigen und geklumpten Punktprozessen durchgeführt (zunächst mit $k=3$ ). Dabei ging es auch um einen Vergleich mit dem, ,angle test“" (Version A3N2) von AsSUNÇÃo und REIS (2000), der ebenfalls auf den Winkeln zwischen den drei nächsten Bäumen basiert, jedoch die exakten Winkelwerte benötigt und somit einen höheren Meßaufwand erfordert.

Die geklumpten Punktverteilungen wurden mit dem Thomas Prozeß (DigGLE et al. 1976) innerhalb der Fläche $\mathrm{D}=[0,50]$ x [0,50] erzeugt, wobei Stichprobenpunkte nur innerhalb der Unterfläche $\mathrm{D}^{*}=[5,45]$ x $[5,45]$ zugelassen wurden, um Randeffekte auszuschließen. In der Simulation wurden die Parameter $\mu$ (mittlere Anzahl Bäume je Klumpen) und $\sigma$ (Standardabweichung der bimodalen Normalverteilung der Abstände der Bäume vom Klumpenzentrum) variiert. Die Dichte (Häufigkeit) der einem Poisson Prozeß folgenden Verteilung der Klumpenzentren wurde mit dem Parameter $\lambda=0.2$ bestimmt. Die regelmäßigen Punktmuster wurden mit Hilfe des Strauss Prozeß (STRAuss 1975) in der Fläche $D=[0,70]$ x $[0,70]$ 
bzw. der Unterfläche $D^{*}=[10,60] \times[10,60]$ erzeugt. Die Stammzahl wurde bei 200 fixiert und der "Abstoßungs-Radius" $r$ wurde auf 4 gesetzt. Der Parameter $\rho$ wurde zwischen 0.0 und 0.8 variiert, wobei $\rho=1$ einen Poisson Prozeß simuliert und $\rho=0$ ein Muster erzeugt, in dem jeder Baum mindestens $r$ Einheiten von seinem nächsten Nachbarn entfernt ist (für Details s. ASSUNÇÃO und REIS 2000).

Für jeden Parametersatz dieser beiden Punktprozesse wurden 500 Realisationen erzeugt und in jedem dieser Punktmuster wurde eine systematische Stichprobe mit 15 (Strauss Prozeß) bzw. 25 (Thomas Prozeß) Probepunkten simuliert (nach Assunção und REIS (2000) sollen diese Stichprobenumfänge sicherstellen, daß die Probepunkte voneinander unabhängig sind). Die Stichprobendaten wurden dann genutzt, um die Teststärke für unterschiedliche kritische Winkel zu berechnen, und zwar innerhalb einer Spanne von $10^{\circ}$ bis $110^{\circ}$, mit einer Schrittweite von $5^{\circ}$. Das Signifikanzniveau des Tests wurde auf 0.05 gesetzt. Abb. 9 zeigt die jeweilige durchschnittliche Stärke des Tests gegen die zwei Alternativhypothesen als Funktion des kritischen Winkels $\alpha_{c}$.

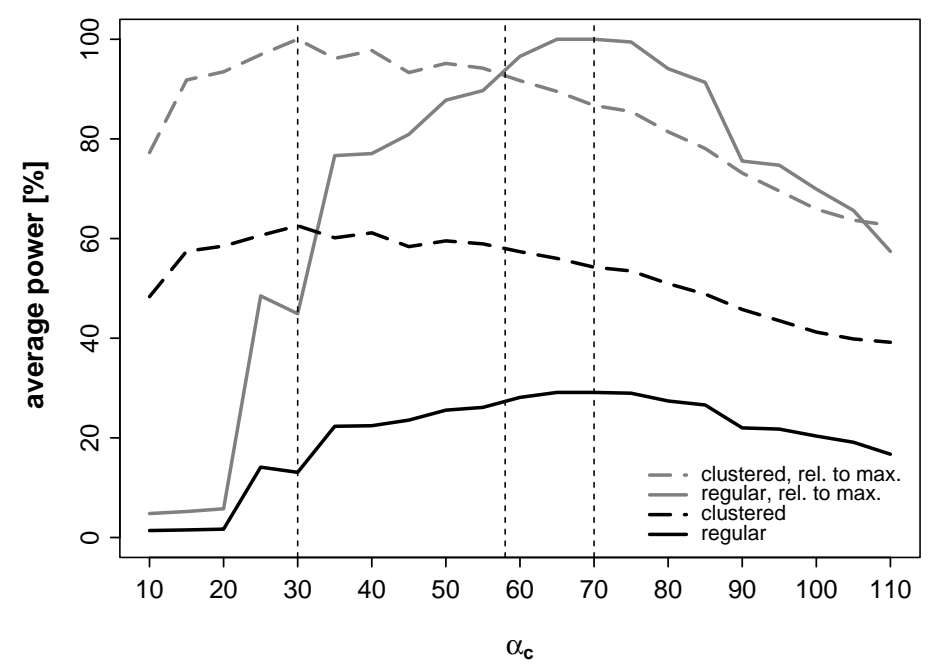

Abb. 9. Die Stärke des $W_{P}$-Tests im Durchschnitt aller simulierten geklumpten bzw. regelmäßigen Verteilungsmuster in Abhängigkeit vom kritischen Winkel $\alpha_{c}$, absolut und in Prozent des jeweils maximalen Wertes. Bei den geklumpten Verteilungen wird die größte Teststärke mit $\alpha_{c}=30^{\circ}$ erreicht, bei den regelmäßigen Verteilungen mit $\alpha_{c}=70^{\circ}$. Wenn keine spezifische Alternativhypothese formuliert wird (werden kann), stellt $\alpha_{c} \approx 60^{\circ}$ einen guten Kompromiß dar.

Um den kritischen Winkel $\alpha_{c}$ bestimmen zu können, der den besten Kompromiß im Fall einer unspezifischen Alternativhypothese darstellt, sind die Teststärken für jede der beiden spezifischen Alternativhypothesen auch standardisiert dargestellt (in Prozent des jeweils maximalen Wertes). Bei Klumpung führt $\alpha_{c}=30^{\circ}$ zur größten Teststärke, während bei Regelmäßigkeit $\alpha_{c}=70^{\circ}$ die Teststärke maximiert. Ist nicht bekannt oder feststellbar, gegen welche der beiden entgegengesetzten Alternativen getestet werden soll, stellt der Schnittpunkt der Linien der standardisierten Teststärken, $\alpha_{c} \approx 60^{\circ}$, einen guten Kompromiß dar (s. Tab. III-1 für eine Liste der zugehörigen kritischen $T$-Werte). Die Grafik zeigt darüber hinaus, daß in regelmäßi- 
gen Punktmustern die Sensitivität der Teststärke gegenüber $\alpha_{c}$ ausgeprägter ist als bei geklumpter Verteilung.

Die detaillierte Betrachtung der Teststärken (s. Abb. III-5) ergab, daß im Fall eines Tests gegen Regelmäßigkeit und bei Anwendung des optimalen $\alpha_{c}$ die Stärke des $W_{P}$-Tests absolut vergleichbar ist mit derjenigen des Tests A3N2 von ASSUNÇÃO und REIS (2000). Bei geklumpten Verteilungen allerdings schneidet der $W_{P}$-Test eindeutig schlechter ab, wenn die Klumpen sehr klein sind $(\mu \leq 1)$. Von diesem Fall abgesehen, sind die Teststärken jedoch ähnlich. Berücksichtigt man die Inventurkosten, läßt sich daraus folgern, daß der $W_{P}$-Test eine interessante Alternative zum Test von ASSUNÇÃO und REIS darstellt, da er keine exakten Winkelmessungen benötigt und damit einen geringeren Inventuraufwand erfordert. Ein exakter Effizienzvergleich wäre allerdings nur auf der Basis von Arbeitszeitstudien möglich.

Das oben beschriebene Verfahren zur Bestimmung des optimalen kritischen Winkels wurde auch für sechs Bäume durchgeführt $(k=6)$, der optimale kritische Winkel $\alpha_{c}$ für die unspezifische Alternativhypothese „Die Verteilung ist entweder regelmäßiger oder geklumpter als bei Zufallsverteilung“ beträgt in diesem Fall $35^{\circ}$. Da das Winkelmaß im Rahmen der 6-Baum-Stichprobe immer auf sechs Bäumen basieren und es im Sinne einer unkomplizierten Anwendung unnötig sein sollte, eine spezifische Alternativhypothese festzulegen, muß das Winkelmaß in der praktischen Anwendung der modifizierten 6-BaumStichprobe also immer mit dem kritischen Winkel $\alpha_{c}=35^{\circ}$ bestimmt werden, da auch alle in den folgenden Kapiteln beschriebenen Berechnungen und Modelle auf diesem Wert beruhen.

\subsubsection{Schätzung der Stammzahl und Grundfläche pro Hektar}

Den Ausführungen von JONSSON et al. (1992) zufolge existiert für die 6-Baum-Stichprobe bei vollständig zufälliger Verteilung der Bäume (Poisson-Wald) ein auch theoretisch erwartungstreuer Schätzer für die Stammzahl pro ha. Dieser lautet ${ }^{7}$

$$
\hat{N}_{h a}=\frac{10000}{\pi \cdot n} \cdot \sum_{i=1}^{n} \frac{5}{r_{i}^{2}}
$$

Um den Bias dieses Schätzers in Beständen mit regelmäßiger oder geklumpter Verteilung und dessen Korrelation mit dem punktbezogenen Winkelmaß quantifizieren zu können, wurden wiederum Stichprobensimulationen durchgeführt. Da für die hier interessierende Fragestellung Baumverteilungsmuster von extremer Regelmäßigkeit bis zu extremer Klumpung unter-

\footnotetext{
${ }^{7}$ Die allgemeine Form dieses Schätzers für jede beliebige Anzahl Bäume je Stichprobeneinheit, bezeichnet mit $k$, lautet: $\hat{N}_{h a}=\frac{10000}{\pi \cdot n} \cdot \frac{k-1}{k} \cdot \sum_{i=1}^{n} \frac{k}{r_{i}^{2}}$ (vgl. a. LESSARD et al. 2002).
} 
sucht werden sollten, war es naheliegend, Bestände mit Hilfe von Punktprozessen (STOYAN und StOYAn 1992) künstlich zu erzeugen. Da allein die Verteilung der Baumpositionen relevant war, war die Anwendung von nicht markierten Punktprozessen ausreichend; alle anderen Baumparameter (Baumart und BHD) wurden also konstant gehalten.

Die generierten Bestände haben jeweils die Flächengröße $A=100 \mathrm{~m}$ x $100 \mathrm{~m}=1$ ha. Die Vorgabe bei der Generierung war die Erzeugung von 1000 Bäumen, die aber in Abhängigkeit von Zufallseffekten und vom gewählten Punktprozeß i.d.R. nicht exakt realisiert wurde. Bei allen Punktprozessen wurden die zugehörigen Parameter so variiert, daß das gesamte Spektrum von extrem regelmäßigen bis zu extrem geklumpten Verteilungsmustern möglichst gleichmäßig abgedeckt wurde. Bei der Generierung der Bestände mit regelmäßiger Baumverteilung kamen vier verschiedene Punktprozesse zur Anwendung, wobei jeweils 10 Bestände erzeugt wurden:

- Randomly Displaced Lattice (RDL, vgl. StOYAn und StOYAn 1992, S. 307 ff.).

- Simple Sequential Inhibition (SSI)

- Strauss Process (STR)

- Matérn Hard Core, Typ II (MHC)

Für die Erzeugung der geklumpten Verteilungsmuster wurden drei verschiedene Punktprozesse genutzt, die alle Spezialfälle des Neyman-Scott-Prozesses darstellen. Hierbei werden in einem ersten Schritt „Elternpunkte“ erzeugt, deren Verteilung einem homogenen PoissonProzeß mit der Intensität $\lambda$ folgt. Jeder „Elternpunkt“ wird dann durch einen Klumpen mit $n_{c}$ Bäumen ersetzt, wobei $n_{c}$ eine Poisson $(\mu)$-verteilte Zufallszahl ist. Damit bei variierender Klumpengröße im Gesamtbestand konstant 1000 Bäume erzeugt wurden, wurde die Intensität des Poisson-Prozesses in Abhängigkeit von $\mu$ gewählt, mit $\lambda=(1000 / \mu) / A$. Die Positionierung der Bäume innerhalb der Klumpen ist abhängig vom jeweiligen Punktprozeß:

- Modified Thomas (MTH $\rightarrow 20$ Bestände)

- Matérn Cluster (MCL $\rightarrow 20$ Bestände)

- SSI Cluster (MIX $\rightarrow 27$ Bestände)

Bis auf die RDL-Punktmuster, die mit Hilfe des Programms STG von STOYAN (1995) generiert wurden, wurden alle anderen mit den entsprechenden Funktionen der Pakete Spatial (VENABLES und RIPLEY 2002) und Spatstat (BADDELEY und TURNER 2006) des Statistikprogramms $R$ (R DEVELOPMENT CORE TEAM 2005) erzeugt (für eine genaue Beschreibung der Punktprozesse s. Abschnitt II-3.3).

In jedem dieser 107 Bestände wurde eine 6-Baum-Stichprobe mit 50 Stichprobenpunkten 1000 mal wiederholt. Die Probepunkte wurden mit Hilfe eines quadratischen Gitternetzes über die Bestandesfläche verteilt, wobei Startpunkt und Ausrichtungswinkel bei jeder Wiederholung zufällig gewählt wurden (systematische Stichprobe mit Zufallsstart). Eine systema- 
tische Unterschätzung der Stammzahl wird durch die Probekreise verursacht, die den Bestandesrand schneiden, da außerhalb der Bestandesfläche keine Bäume vorhanden sind bzw. erfaßt werden. Ein anerkanntes Verfahren zur Korrektur dieses Randfehlers ist die Spiegelungsmethode nach SCHMID-HAAS (1969b), die jedoch an Bestandesecken, wo der Probekreis von mehr als einer Bestandesgrenze geschnitten wird, nur unvollständig möglich ist (Pommerening 1997, S. 41). Daher wurde stattdessen für jeden am Rand liegenden Probekreis der Anteil der ideellen Probekreisfläche ermittelt, der innerhalb der Bestandesfläche liegt (vgl. BEERS 1966). Zur Hochrechnung der Stammzahl auf ha-Werte wurde dann nur diese Teilfläche $a_{i}^{i n}$ verwendet (s. Formel (8), in diesen Fällen ist also $r_{i}=\sqrt{a_{i}^{i n} / \pi}$ ).

Für die praktische Durchführung der Simulation wurde unter Borland Delphi (Programmiersprache Pascal) der Stichprobensimulator PoiSSim entwickelt (STAUPENDAHL 2001). Dieses Programm simuliert die wiederholte Stichprobenziehung in einem Bestand und speichert nach jedem Simulationsdurchgang die geschätzte Stammzahl pro ha, das Winkelmaß und weitere Bestandes- und Stichprobenparameter, so daß am Ende der Simulation die Erwartungs- und Biaswerte berechnet werden können. Der Erwartungswert eines Parameterschätzers $\hat{\theta}$ ist der Mittelwert der Schätzergebnisse für alle möglichen Stichproben $r$, mit $r=$ $1 . . m$ und $m \rightarrow \infty$. Mit der hier gewählten Anzahl an Stichprobenwiederholungen $(m=1000)$ wird eine ausreichend genaue Schätzung erreicht. Der Bias ist dann die Differenz aus Erwartungswert und wahrem Wert: $\operatorname{Bias}(\hat{\theta})=E(\hat{\theta})-\theta$. Zur Analyse der resultierenden Daten wurde das Statistik-Programm $R$ (R DeVELOPMENT CoRE TEAm 2005) verwendet.

Die Simulationsdaten zeigten, daß das punktbezogene Winkelmaß $W_{P}$ in einem sehr straffen, nichtlinearen Zusammenhang zum relativen Bias der Stammzahlschätzung steht (Abb. 10). Als gut angepaßtes, aber dennoch einfaches Modell zur Beschreibung dieses Zusammenhangs erwies sich die Gleichung

$$
\begin{aligned}
& \operatorname{rias}\left(\hat{N}_{h a}\right)=c_{1}+c_{2} \cdot E\left(\hat{W}_{P}\right)^{c_{3}}, \\
& \operatorname{mit} \operatorname{rBias}\left(\hat{N}_{h a}\right)=\frac{E\left(\hat{N}_{h a}\right)-N_{h a}}{N_{h a}}=\frac{E\left(\hat{N}_{h a}\right)}{N_{h a}}-1 .
\end{aligned}
$$

Die mit Hilfe der nichtlinearen Regressionsrechnung ermittelten Koeffizienten sind in Tab. 1 wiedergegeben.

Tab. 1: Koeffizienten der Regressionsgleichung zur Schätzung des bei der 6-Baum-Stichprobe zu erwartenden relativen Bias der Stammzahl pro ha.

\begin{tabular}{cccc}
\hline Koeffizient & Wert & Standardfehler & Signifikanzniveau \\
\hline$c_{1}$ & $-0,075402$ & 0,005827 & 0,001 \\
$c_{2}$ & 1,358629 & 0,035418 & 0,001 \\
$c_{3}$ & 3,109408 & 0,092690 & 0,001 \\
\hline
\end{tabular}


Jeder der Koeffizienten des Modells ist hochsignifikant und mit 97,7 \% wird ein hoher Anteil der Streuung der Biaswerte erklärt. Wichtig ist vor allem der geringe Standardfehler der Residuen: $95 \%$ liegen innerhalb eines Intervalls von $\pm 5,2 \%$ und damit in einer Größenordnung, die für praktische Zwecke kaum von Bedeutung ist. Dabei gilt es zu bedenken, daß die Residuenstreuung in den stark geklumpten Beständen am stärksten ist (s. Abb. 10) und hier Bestände mit einem Klumpungsgrad erzeugt wurden, der in natura nur in den seltensten Fällen zu erwarten ist.

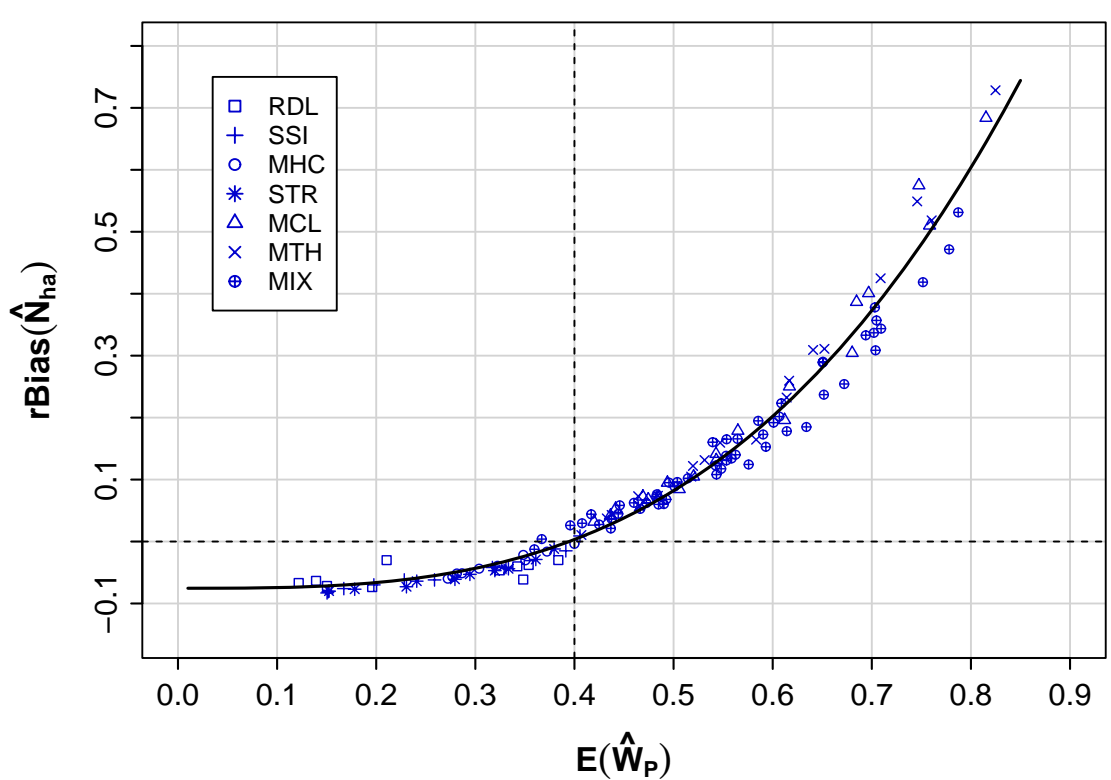

Abb. 10. Bias der Stammzahl pro ha bei der 6-Baum-Stichprobe (relativ zur wahren Stammzahl) in Abhängigkeit vom punktbezogenen Winkelmaß $W_{P}$ (mit $k=6$ und $\alpha_{c}=35^{\circ}$, s. Kap. 4.3.1). Die verschiedenen Punktsymbole bezeichnen die Punktprozesse, mit denen die zugehörigen Simulationsbestände erzeugt wurden, die Kurve beschreibt Regressionsgleichung (9), mit der die Punktewolke rechnerisch ausgeglichen wurde. Die vertikale punktierte Linie markiert den $W_{P}$-Wert eines „Poisson-Waldes“, bei dem im Mittel kein Bias auftritt.

Gemäß Gleichung (9) gilt

$$
\begin{aligned}
\frac{E\left(\hat{N}_{h a}\right)}{N_{h a}}-1 & =c_{1}+c_{2} \cdot E\left(\hat{W}_{P}\right)^{c_{3}} \\
\Leftrightarrow \quad N_{h a} & =\frac{E\left(\hat{N}_{h a}\right)}{1+c_{1}+c_{2} \cdot E\left(\hat{W}_{P}\right)^{c_{3}}},
\end{aligned}
$$

so daß der in Nicht-Poisson-Wäldern zu erwartende Bias des Schätzers (8) demnach auf ein vertretbares Maß reduziert werden kann, wenn die Schätzung aus (8) durch $1+\operatorname{rBias}\left(\hat{N}_{h a}\right)$ dividiert wird. Der neue Schätzer der 6-Baum-Stichprobe für die Stammzahl pro ha lautet dann 


$$
\hat{N}_{h a}=k f \cdot \frac{10000}{\pi \cdot n} \cdot \sum_{i=1}^{n} \frac{5}{r_{i}^{2}}, \text { mit } k f=\frac{1}{0,9246+1,3586 \cdot \hat{W}_{P}^{3,109}} .
$$

Aus Formel (10) ist allerdings auch ersichtlich, daß die Präzision der Schätzung des $W_{P}$-Wertes bei der Biaskorrektur eine wichtige Rolle spielt. Denn wenn $\hat{W}_{P}$ stark streut, wird der Korrekturfaktor im Mittel zwar korrekt sein, im Einzelfall den Bias aber über- oder unterschätzen, was je nach Vorzeichen des tatsächlichen Bias sogar zu einer Vergrößerung des Schätzfehlers führen kann (s. Kap. 4.3.4). Daneben fällt in Abb. 10 auf, daß der Zusammenhang zwischen Winkelmaß und Bias in den Beständen mit RDL-Punktmustern (gitternetzartiger Anordnung der Bäume) deutlich weniger straff ist. Bereits JONSSON et al. (1992) hatten beobachtet, daß der Stammzahlbias mit der Kantenlänge von gitternetzartigen Verteilungsmustern variiert. Offensichtlich kann diese Varianz mit dem Winkelmaß nur ungenügend erklärt werden. Somit wäre die Biasschätzung in Beständen, die künstlich durch Reihenpflanzung begründet wurden und deren Reihenstruktur noch deutlich erkennbar ist, mit Unsicherheiten behaftet. Allerdings ist diese Einschränkung nicht sehr schwerwiegend, da der in solchen Beständen zu erwartende Bias nicht sehr hoch zu sein scheint.

Desweiteren läßt die Streuung der Wertepaare im Bereich geklumpter Verteilungsmuster vermuten, daß bei gleichem $W_{P}$-Wert doch deutlich unterschiedliche Verteilungsmuster vorliegen können, die dann auch unterschiedliche systematische Fehler erzeugen; eine Vermutung, die durch die optische Überprüfung von Beständen mit fast identischem $W_{P}$-Wert bestätigt wird (vgl. Abb. II-5). Dies bedingt, daß mit dem hier entwickelten Modell z. B. für Bestände mit Verteilungsmustern, die dem „MIX“-Punktprozeß entsprechen, bei $W_{P}$-Werten $<0,5$ mit einer systematischen Unterschätzung, bei $W_{P}$-Werten darüber mit einer Überschätzung des Bias zu rechnen ist. Ob diese systematischen Fehler allerdings von praktischer Bedeutung sind, wird im Rahmen der späteren Validierung des Modells (Kap. 4.3.4) geklärt.

Die Schätzung der Grundfläche pro ha kann in die Komponenten Stammzahl pro ha und mittlere Baumgrundfläche zerlegt werden (vgl. JONSSON et al. 1992). Der Schätzer für die Grundfläche pro ha lautet dann

$$
\hat{G}_{h a}=k f \cdot \frac{10000}{\pi \cdot n} \cdot \sum_{i=1}^{n} \frac{5}{r_{i}^{2}} \cdot \bar{g}_{i}, \text { mit } \bar{g}_{i}=\frac{\pi}{40000 \cdot 6} \cdot \sum_{j=1}^{6} d_{i j}^{2},
$$

wobei $d_{i j}$ die Brusthöhendurchmesser der Bäume des $i$-ten Probekreises bezeichnet. SABOROWSKI und ŠMELKO (1998) konnten anhand einer stichprobentheoretischen Analyse und deren experimenteller Überprüfung zeigen, daß das arithmetische Mittel aus den auf Hektar hochgerechneten Probekreis-Grundflächen bei systematischen Stichproben mit variablen Probeflächengrößen ein erwartungstreuer Schätzer ist. Die dort in der theoretischen Beweisführung unterstellte vollständige Aufteilung des Inventurgebiets in sich nicht überlappende, quadratische Probeflächen stellt allerdings nur eine Approximation an den tatsächlichen 
Stichprobenraum der 6-Baum-Stichprobe dar, da es - wie die Autoren selber anführen - unendlich viele mögliche Stichprobeneinheiten gibt (vgl. a. KLEINN und VILČKO 2005). Weitere Probleme, die im Zusammenhang mit Schätzer (11) auftreten können, und Ansätze zu deren Lösung werden im folgenden Kapitel behandelt.

\subsubsection{Aufnahmetechnische Modifikationen}

Da die Korrektur der verzerrten Stammzahlschätzung nur gesamthaft für den Mittelwert, nicht aber ,probekreis-individuell““ erfolgt, kann bei der Schätzung der Grundfläche pro ha gemäß Gleichung (11) ein Bias dadurch entstehen, daß einzelne Probekreise aufgrund der variablen Flächengrößen ein zu hohes oder zu niedriges Gewicht erhalten. HIRNER (1978, S. 56 f.) beschrieb die Überlagerung der Standflächen mehrerer Einzelbäume in mehrschichtigen Beständen, wie sie z. B. in zweischichtigen Buchenbeständen mit stammzahlreichem Unterstand auftreten, als eine mögliche Ursache für solche Effekte. Wollte man einen solchen Bestand in Gänze mit der 6-Baum-Stichprobe erfassen, würden sehr viele unterständige Buchen aufgenommen, während die wertvollen Stämme des Hauptbestandes nur mit geringer Stammzahl in die Stichprobe eingingen. Neben dem dadurch evtl. erzeugten systematischen Fehler wären also die Schätzungen für den Unterstand unnötig präzise und für den Hauptbestand zu unpräzise.

Auf der Ebene von permanenten Probekreisinventuren führten diese Überlegungen zur Einführung konzentrischer Probekreise (z. B. STARKE 1989). Als Konsequenz für die 6Baum-Stichprobe und nach dem Vorbild der im Baden-Württembergischen Betriebsinventurverfahren vorgesehenen, sogenannten doppelten 6-Baum-Stichprobe (BLUST et al. 2006) wird hier eine Stratifizierung nach Bestandesschichten ${ }^{8}$ vorgeschlagen (s. Abb. 11). Demnach werden bei der Auswahl der sechs nächsten Bäume im Rahmen der modifizierten 6-BaumStichprobe nur die Bäume des Hauptbestandes berücksichtigt! An einem Stichprobenpunkt $i$ evtl. vorkommender Überhalt oder Unterstand (Schichten des Nebenbestandes) wird dagegen durch eine okulare Schätzung der Stammzahl $n_{N B, i}$ und des Durchmessers des Grundflächenmittelstamms $d g_{N B, i}$ innerhalb eines festen Radius $r_{N B, i}$ von 6 m (im Unterstand) bzw. 15 m (im Überhalt) erfaßt. Dabei wird davon ausgegangen, daß an einem einzelnen Stichprobenpunkt zusätzlich zum Hauptbestand nur eine dieser beiden Schichten vorkommt. In Beständen ohne eindeutig abgrenzbare Schichten mit exponentiell abnehmender Durchmesserverteilung (z. B. in Plenterwäldern) sollte durch Festlegung eines Grenzdurchmessers ein Hauptbestand und Unterstand definiert werden, wobei dieser Durchmesser so zu wählen wäre, daß beide

\footnotetext{
8 Nach der niedersächsischen Forsteinrichtungsanweisung (NIEDERSÄCHSISCHE LANDESFORSTEN 1987) sind die Bestandesschichten folgendermaßen definiert: Hauptbestand: Bestandesschicht, auf der das wirtschaftliche Hauptgewicht liegt; Überhalt: Reste aus dem Vorbestand über dem Hauptbestand mit einem Bestockungsgrad $\leq$ 0,3; Unterstand: Unter dem geschlossenen Hauptbestand wachsender Baum- und Strauchwuchs, [...].
} 
„Schichten“ möglichst gut getrennt werden, um die Zahl der Bäume zu minimieren, bei denen unklar ist, zu welchem Kollektiv sie gehören.

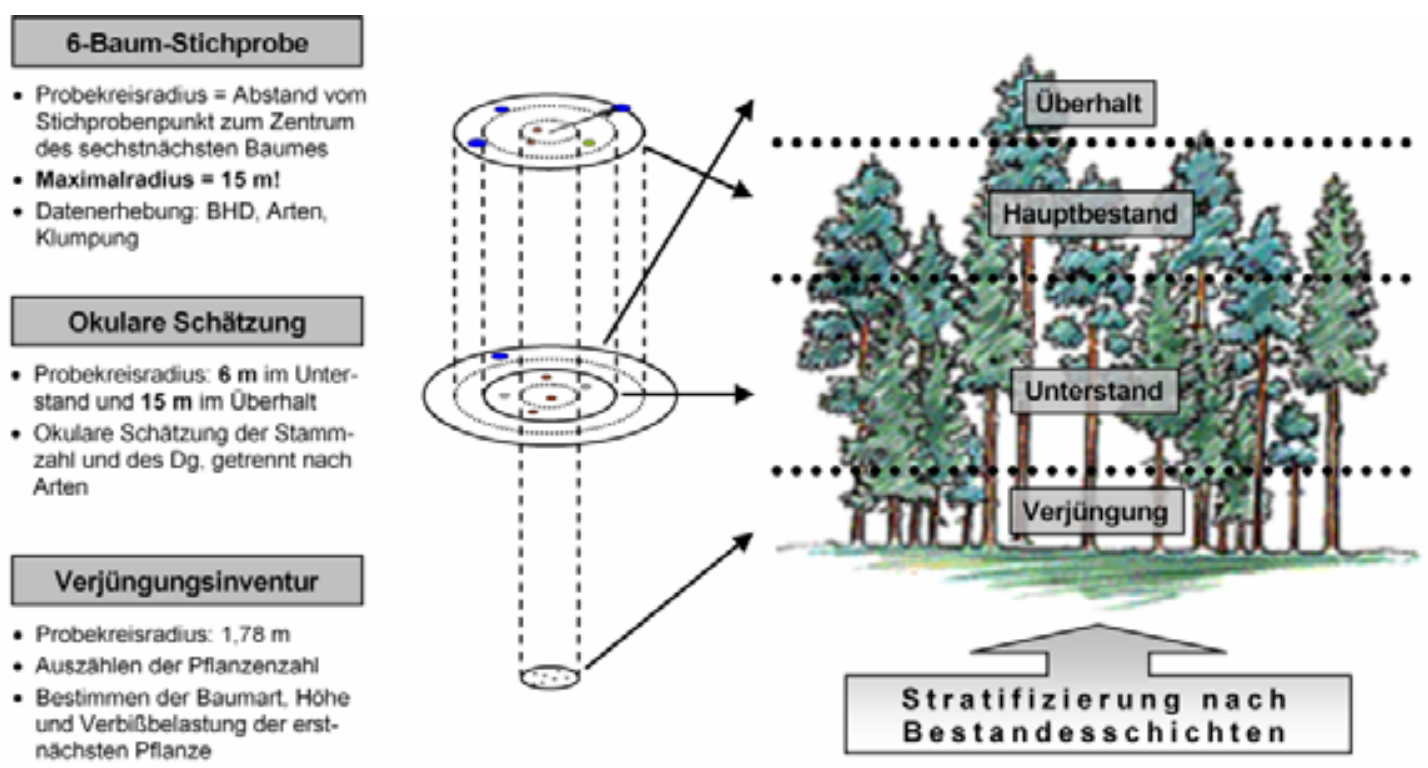

Abb. 11. Schematische Darstellung der Stratifizierung eines Bestandes nach Schichten und der sich daraus ergebenden Inventurarbeiten am Stichprobenpunkt. Um das Verfahren in seiner Vollständigkeit demonstrieren zu können, ist hier ein Fall konstruiert, in dem im Nebenbestand Unterstand und Überhalt gleichzeitig vorkommen. Dies kann für die Praxis ausgeschlossen werden, so daß nie mehr als maximal drei Probekreise aufzunehmen sind (zur Stratifizierung in Plenterbeständen s. Anm. im Text).

Ein weiterer wichtiger Aspekt ist die Begrenzung des Probekreisradius, der bei der 6Baum-Stichprobe normalerweise durch den Abstand des sechstnächsten Baumes zum Probekreismittelpunkt definiert ist. HIRNER (1978, S. 70) wies bereits darauf hin, daß in schwach bestockten Bestandespartien und auf Blößen die Durchführung der 6-Baum-Stichprobe sehr erschwert wird, da aufgrund der dort großen Meßdistanzen die Ermittlung des sechsten Baumes sehr zeitaufwendig ist, wodurch der Vorteil des Verfahrens, nämlich die einfache und rasche Merkmalserhebung, wieder hinfällig wird. Deshalb werden in der modifizierten 6Baum-Stichprobe nur diejenigen Bäume des Hauptbestandes berücksichtigt, die maximal $\mathbf{1 5} \mathbf{m}$ vom Stichprobenpunkt entfernt sind.

In Bereichen mit geringer Bestandesdichte findet also ein Wechsel von Probekreisen mit variablem Radius und fester Stammzahl $\left(n_{i}=6\right)$ zu Probekreisen mit festem Radius und variabler Stammzahl statt $\left(0 \leq n_{i} \leq 5\right)^{9}$. In beiden Fällen werden die flächenbezogenen Schätzgrößen zunächst für jeden Probekreis auf Hektar-Werte hochgerechnet und anschließend über alle Probekreise arithmetisch gemittelt. Ein systematischer Fehler der Stammzahlschätzung ist nur für die Probekreise mit variablem Radius zu erwarten, so daß das oben beschriebene Kor-

\footnotetext{
${ }^{9}$ Bei größeren Blößen ist es natürlich sinnvoll, diese vor der Inventur (z. B. Mit Hilfe eines Luftbildes) zu ermitteln und bei der Stichprobennahme unberücksichtigt zu lassen. Dies beschleunigt die Meßarbeiten erheblich und kann den Stichprobenfehler deutlich reduzieren.
} 
rekturverfahren auch nur auf diesen Teil der Stichprobe angewendet wird. Die neuen Schätzer für die Stammzahl und Grundfläche pro ha lauten dann

$$
\begin{aligned}
& \hat{N}_{h a}=\frac{10000}{\pi \cdot n} \cdot \sum_{\mathrm{i}=1}^{\mathrm{n}} \frac{n_{i}^{*}}{r_{i}^{2}}+\frac{n_{N B, i}}{r_{N B, i}^{2}} \text { und } \\
& \hat{G}_{h a}=\frac{10000}{\pi \cdot n} \cdot \sum_{\mathrm{i}=1}^{\mathrm{n}} \frac{n_{i}^{*}}{r_{i}^{2}} \cdot \bar{g}_{i}+\frac{n_{N B, i}}{r_{N B, i}^{2}} \cdot \bar{g}_{N B, i}, \\
& \text { mit } \quad n_{i}^{*}= \begin{cases}n_{i} & \text { falls } n_{i}<6 \\
\frac{0,9246+1,3586 \cdot \hat{W}_{P}^{3,1094}}{n_{P}} & \text { falls } n_{i}=6\end{cases} \\
& \bar{g}_{i}=\frac{\pi}{40000 \cdot n_{i}} \cdot \sum_{j=1}^{n_{i}} d_{i j}^{2} \\
& \bar{g}_{N B, i}=\frac{\pi}{40000} \cdot d g_{N B, i}^{2}
\end{aligned}
$$

Bei der Schätzung des Winkelmaßes $W_{P}$ gemäß Formel (7) bleiben neben den Probekreisen, die den Bestandesrand schneiden, nun natürlich auch die Probekreise mit weniger als 6 Bäumen unberücksichtigt! Der durch die okulare Schätzung im Nebenbestand zusätzlich entstehende Fehler, sowohl zufälliger als auch systematischer Art, dürfte nach einiger Übung in vertretbaren Grenzen zu halten sein, zumal die absolute und/oder relative Höhe der Grundfläche (als i.d.R. maßgebliche meßbare Variable einer Bestandesinventur) im Nebenbestand fast immer sehr gering ist ${ }^{10}$. Dagegen ist zu erwarten, daß der Hauptbestand, der den Hauptanteil des Bestandesvorrates bildet, um so genauer geschätzt wird, so daß der Fehler der Vorratsschätzung des Gesamtbestandes je nach Bestandesstruktur z. T. erheblich geringer sein dürfte als bei der Variante ohne Stratifizierung.

Die Höhenmessung ist die zeitaufwendigste Arbeit bei der Datenerhebung. Um dennoch für jeden Baum das Stamm- bzw. Derbholzvolumen berechnen zu können (NAGEL 1988), werden die Höhen der Einzelbäume getrennt nach Bestandesschichten mit Hilfe einer Einheitshöhenkurve geschätzt (KRAMER und AKÇA 1995, S. 138). Die als Eingangsgröße hierfür benötigte Mittelhöhe sollte für jede Hauptbaumart des Hauptbestandes über ca. 5 Höhenmessungen an Bäumen mit einem BHD im Bereich des Durchmessers des Grundflächenmittelstamms ermittelt werden. Für selten vorkommende Arten des Hauptbestandes sowie für die Baumarten des Nebenbestandes sollte eine gutachtliche Schätzung der Mittelhöhe ausreichend sein.

\footnotetext{
10 Zur Kalibrierung und Kontrolle der Schätzung sollten allerdings in regelmäßigen Abständen einige Bäume gekluppt und Grenzstämme durch Abstandsmessung kontrolliert werden.
} 
Desweiteren sollte eine Bestandesinventur auch den Verjüngungszustand erfassen, zumal ein großer Anteil der Arbeitszeit allein auf das Aufsuchen der Stichprobenpunkte entfällt und zuverlässige Informationen über die Verjüngung in anspruchsvollen Waldbaukonzepten von besonderer Bedeutung sind. Es erscheint also sinnvoll, an jedem Stichprobenpunkt möglichst vielfältige Informationen zu erheben. Da das Arbeitsvolumen aber in einem vertretbaren Rahmen bleiben muß, wird für die Erfassung der Verjüngungssituation das Verfahren nach STAUPENDAHL (1997) vorgeschlagen, das besonders einfach und schnell anzuwenden ist. Tab. 2 zeigt eine Übersicht über alle an einem Stichprobenpunkt zu erhebenden Variablen.

Tab. 2: Übersicht über die am Stichprobenpunkt - getrennt nach Bestandesschichten - zu erfassenden Variablen. Höhe und Kronenansatz werden natürlich nicht an jedem Probebaum gemessen (s. Text). Zur Korrektur des Randfehlers muß der Abstand vom Probekreismittelpunkt zum Bestandesrand gemessen werden, wenn sich Grenzlinie und Probekreis schneiden (vgl. BEERS 1966 und Abschnitt II-3.3). Das Winkelmaß ist nur dann zu ermitteln, wenn tatsächlich sechs Bäume im Probekreis der 6-Baum-Stichprobe liegen. Optional können bei den Probebäumen weitere Merkmale erhoben werden (z. B. die Güteklasse des Erdstammstückes). Die Verwendung eines mobilen Datenerfassungsgerätes (MDE) hat sich bei Inventur und Datenimport als sehr hilfreich erwiesen.

\begin{tabular}{cccc}
\hline & Probekreis & $\begin{array}{c}\text { Variablen je... } \\
\text { Probebaum }\end{array}$ & Baumart \\
\hline $\begin{array}{c}\text { Hauptbestand } \\
\text { (6-Baum-Stichprobe) }\end{array}$ & $\begin{array}{c}\text { Radius, Winkelmaß, } \\
\text { Abstand vom } \\
\text { Bestandesrand }\end{array}$ & $\begin{array}{c}\text { BHD, Höhe, } \\
\text { Kronenansatz, ... }\end{array}$ & - \\
$\begin{array}{c}\text { Überhalt / Unterstand } \\
\text { (okulare Schätzung) }\end{array}$ & $\begin{array}{c}\text { Abstand vom } \\
\text { Bestandesrand }\end{array}$ & - & $\begin{array}{c}\text { Mitteldurchmesser (Dg), } \\
\text { Mittelhöhe (Hg), } \\
\text { Nachwuchs }\end{array}$ \\
(Verjüngungsingent
\end{tabular}

\subsubsection{Validierung}

Im folgenden soll die Güte der entwickelten Schätzer für die Stammzahl und Grundfläche pro ha überprüft werden. Hierfür kam wieder die Stichprobensimulation zum Einsatz, wobei als Datenbasis 27 vollaufgenommene Bestände zur Verfügung standen, die von Plantagen mit sehr regelmäßiger Baumverteilung bis zu Plenterwäldern ein weites Spektrum von Bestandesstrukturen abdecken. Um die Spannweite der Baumverteilungsmuster weiter zu erhöhen und damit allgemeingültigere Interpretationen zu ermöglichen, wurde zusätzlich jeder Bestand dupliziert und anschließend virtuell „,durchforstet“, indem alle Bäume entfernt wurden, die sich innerhalb von zufällig im Bestand verteilten Kreisflächen mit einem Radius von $10 \mathrm{~m}$ befanden. Die Anzahl der als Femel- oder Störungsflächen interpretierbaren Entnahmeplots je Bestand wurde dabei durch eine zwischen 5 und 20 gleichverteilte Zufallszahl bestimmt. Für die Validierung standen somit insgesamt 54 Bestandesdatensätze zur Verfügung (vgl. Abb. II-6 u. II-7). 
Um die durch die stufenweisen Modifikationen des Verfahrens vermutete Verbesserung der Schätzgenauigkeit überprüfen zu können, wurden alle drei hier behandelten Varianten der 6-Baum-Stichprobe untersucht:

Variante I: Konventionelle 6-Baum-Stichprobe ohne Biaskorrektur (Schätzer (10) und (11) mit $k f=1$ )

Variante II: Konventionelle 6-Baum-Stichprobe mit Biaskorrektur (Schätzer (10) und (11))

Variante III: Modifizierte 6-Baum-Stichprobe mit Biaskorrektur, begrenztem Probekreisradius und nach Bestandesschichten differenzierter Inventurmethodik (Schätzer (12) und (13))

Für jede dieser Varianten wurden in jedem der Validierungsbestände mit Hilfe des Stichprobensimulators PoiSSim Stichproben mit 10, 20, 30, 40 und 50 systematisch verteilten Probepunkten simuliert, mit jeweils 1000 Wiederholungen (zur Korrektur der Randfehler s. Kap. 4.3.1 u. 4.3.2). Da die Validierungsdaten keine Informationen über die Zugehörigkeit der Einzelbäume zu Bestandesschichten enthielten, wurde in allen Beständen mit bimodaler oder exponentieller Durchmesserverteilung ( $85 \%$ aller Validierungsdatensätze) die in Variante III vorgesehene schichtenabhängige Stratifizierung durch einen bestandesindividuellen Grenzdurchmesser vorgenommen, oberhalb dessen ein Baum zum Hauptbestand und unterhalb dessen er zum Nebenbestand (Unterstand) zählt. Die Bestimmung dieses Durchmessers erfolgte gutachtlich anhand der Durchmesserverteilung; bei bimodalen Verteilungen entsprach er dem Minimum zwischen den beiden Häufigkeitsmaxima (vgl. a. HESSENMÖLlER und GADOW 2001), bei exponentiellen Verteilungen dem Durchmesser, bei dem die Abnahme der KlassenHäufigkeit deutlich abflacht, also beim Übergang vom Schwach- zum Mittelholz (vgl. SCHÜTZ 2002). Über alle stratifizierten Bestände beträgt er zwischen $14 \mathrm{~cm}$ und $32 \mathrm{~cm}$, im Mittel $19 \mathrm{~cm}$. Um den bei der okularen Schätzung des Mitteldurchmessers und der Stammzahl des Unterstandes zu erwartenden Fehler zu simulieren, wurde den entsprechenden Werten bei jeder Stichprobenziehung ein normalverteilter Streuungsbetrag hinzugefügt, so daß sich ein mittlerer zufälliger Fehler von $\pm 20 \%$ ergab.

Zusätzlich zum Bias wurde für jede Zielgröße neben der Standardabweichung der Stichprobenmittelwerte $S D$ (= Standardfehler) auch die Wurzel aus der mittleren quadratischen Abweichung der Stichprobenmittelwerte vom wahren Populationswert (Root Mean Square Error $=R M S E$ ) berechnet. Der RMSE berücksichtigt neben der Präzision des Parameterschätzers $\hat{\theta}$ auch den systematischen Fehler und läßt sich aus $m$ Simulationsläufen schätzen über $\operatorname{RMSE}(\hat{\theta})=\sqrt{E\left(\hat{\theta}_{r}-E\left(\hat{\theta}_{r}\right)\right)^{2}+\operatorname{Bias}(\hat{\theta})^{2}}$, mit $r=1 . . m$, wobei mit $m=1000$ eine ausreichend genaue Schätzung erreicht wird. Sowohl $S D$, als auch RMSE wurden durch Division durch den wahren Populationswert $\theta$ standardisiert und dann mit $r S D$ bzw. $r R M S E$ bezeichnet.

Die Ergebnisse der Stichprobensimulation lassen sich wie folgt zusammenfassen (vgl. a. Abb. II-8 bis II-11): 
1.) Bereits allein durch die Anwendung des winkelmaßbasierten Korrekturfaktors läßt sich der Bias der Stammzahlschätzung in einem weiten Spektrum von Bestandestypen in einem vertretbaren Rahmen von $\pm 5 \%$ halten, was zur Folge hat, daß der $r R M S E$ als Maß für den Gesamtfehler der Schätzung in 98 \% (Variante II) bzw. 99,8 \% (Variante III) der Fälle unterhalb des 1,1-fachen Standardfehlers bleibt. Dies ermöglicht eine universelle Anwendbarkeit der 6-Baum-Stichprobe, die nicht wie bisher (z. B. PICARD et al. 2005) durch Annahmen zum Baumverteilungsmuster eingeschränkt ist.

Eine statistisch saubere und elegantere Lösung stellt freilich der Ansatz von KLEINN und VILČKO (2006) dar, bei dem die Einschlußflächen mit Hilfe der $k$-order Voronoi Polygone direkt berechnet werden. Auf der Basis der dann bekannten Auswahlwahrscheinlichkeiten der Stichprobenbäume läßt sich mit dem Horwitz-Thompson-Schätzer (HoRwITZ und THOMPSON 1952) jede beliebige Bestandesvariable erwartungstreu schätzen. Allerdings müssen für die Berechnung der Einschlußflächen die Positionen der Stichprobenbäume und zusätzlich - je nach Raumstruktur - evtl. zahlreicher weiterer benachbarter Bäume eingemessen werden. Dies kann, v. a. in dichten Beständen mit Unterstand, selbst mit Laserentfernungsmessern eine aufwendige Prozedur sein, die der Schnelligkeit des Verfahrens zuwiderläuft. Solange diese inventurtechnischen Fragen nicht geklärt sind, könnte also der hier beschriebene Ansatz eine wertvolle Alternative sein.

2.) Durch die Beschränkung der eigentlichen (bias-korrigierten) 6-Baum-Stichprobe auf den Hauptbestand und die Erfassung des Nebenbestandes mittels okularer Schätzung von Mitteldurchmesser und Stammzahl innerhalb fester Probekreise (Variante III) kann auch der Betrag des Bias der Grundflächenschätzung selbst in extrem strukturreichen Beständen auf ein vertretbares Maß von unter $5 \%$ gesenkt werden (der $r R M S E$ beträgt dadurch immerhin noch in 90 \% der Fälle weniger als das 1,1-fache des Standardfehlers). Während der Stichprobenfehler in Variante II im Mittel sogar etwas höher war als in der ursprünglichen 6-BaumStichprobe, was durch den Einfluß der Streuung der Winkelmaßes erklärt werden kann, führt die Vergrößerung der bei der konventionellen 6-Baum-Stichprobe grenzwertig kleinen Stichprobeneinheit $^{11}$ in Variante III zu einer deutlichen Verringerung des Stichprobenfehlers, vor allem bei der Schätzung der Grundfläche pro ha, der in den meisten Fällen wohl wichtigsten meßbaren Variable bei Bestandesinventuren. Auch der Bias der Stammzahlschätzung wird weiter reduziert. Dem gegenüber ist der Mehraufwand im Vergleich zur konventionellen 6Baum-Stichprobe, bedingt durch die schichtenspezifische Genauigkeit der Datenerhebung, sehr gering.

3.) Durch die Begrenzung des Radius der SEBAST-Probekreise auf maximal $15 \mathrm{~m}$ wird die Einfachheit der Datenerfassung im Gelände weiter erhöht. Eine dadurch bedingte Ver11 Die optimale Größe der Stichprobeneinheit wird von AKÇA (2001, S. 38) für deutsche Verhältnisse mit
15 - 20 Bäumen angegeben. 
schlechterung der Schätzgenauigkeit, im Sinne eines systematischen Fehlers, konnte in dieser Untersuchung nicht nachgewiesen werden.

Um die tatsächliche Effizienz des hier vorgestellten Verfahrens im Vergleich zu konkurrierenden Inventurmethoden besser beurteilen zu können, wären Zeitstudien natürlich sehr wünschenswert gewesen. Ebenso wäre es sinnvoll, zu untersuchen, welche Auswirkungen ein systematischer Fehler bei der okularen Schätzung des Unterstandes auf die Schätzung der Stammzahl und Grundfläche des Gesamtbestandes hat. Die Bearbeitung dieser Fragen hätte jedoch den Rahmen dieser Arbeit gesprengt und bleibt somit zukünftigen Untersuchungen vorbehalten. 


\section{Software zur Fortschreibung, Planung und Entscheidungsunterstüt- zung}

Ein forstliches Informationssystem und dynamisches Betriebswerk erlaubt im Gegensatz zu einem traditionellen Forsteinrichtungswerk, flexibel auf unterschiedliche Informationsbedürfnisse des Forstbetriebes zu reagieren. FÄHSER (1983) weist auf die Notwendigkeit einer dynamischen Informationsbereitstellung hin, wenn eine qualitativ hochwertige Forsteinrichtung bzw. Informationserfassung auch zu einer Leistungssteigerung im Betriebsablauf führen soll (vgl. a. BITTER 1990; KÄTSCH 1998). Bereits bei der Aufbereitung der erhobenen Daten lassen sich Effizienz und Marktorientierung der Inventuren deutlich steigern, wenn ein flexibles und leistungsfähiges Berechnungssystem verwendet wird (PUUMALAINEN et al. 1998). Parallel zur Konzeption eines schlüssigen Inventurdesigns sollte daher der Entwicklung eines anwenderfreundlichen und leistungsfähigen Datenbank- und Auswertungsprogramms besondere Aufmerksamkeit geschenkt werden. Abb. 12 illustriert den grundsätzlichen Aufbau der Forstplanungssoftware ARGUS Waldplaner+, deren Prototyp im Rahmen dieser Arbeit in der Programmiersprache Object Pascal (Entwicklungsumgebung Borland Delphi) entwickelt wurde und somit auf die besonderen Anforderungen von Eingriffsinventuren abgestimmt ist.

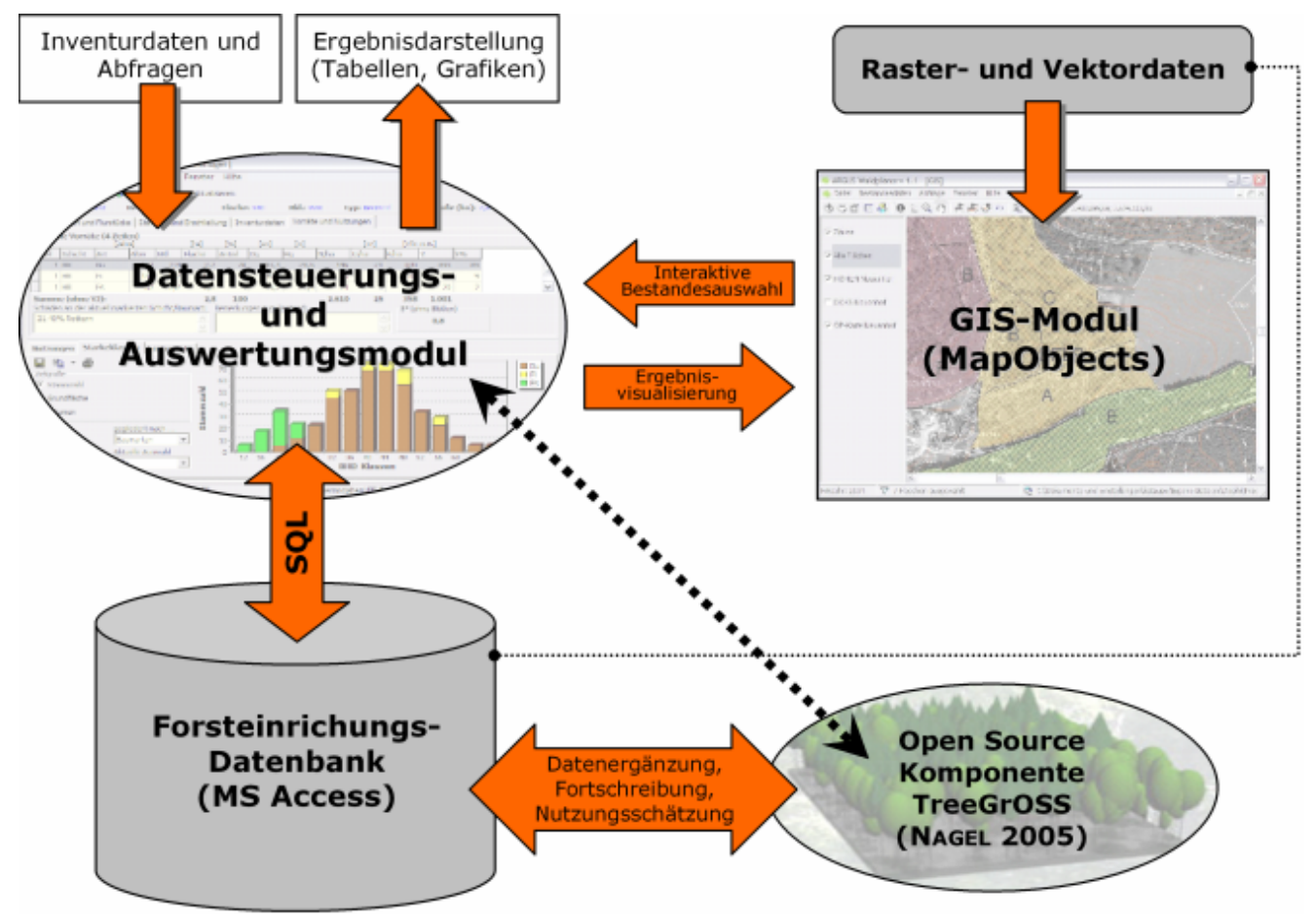

Abb. 12. Struktur des Programms ARGUS Waldplaner+ zur Verarbeitung und Präsentation von Daten aus Eingriffsinventuren.

Zentrales Element des Programms ist ein Datensteuerungs- und Auswertungsmodul, das den Datenimport regelt und benutzerdefinierte Abfragen an die Datenbank weiterleitet und auswertet. Mit Hilfe der von NAGEL (2005) in der Programmiersprache Java entwickelten und in das Programm integrierten Softwarekomponente TreeGrOSS (Tree Growth Open Source 
Software) ${ }^{12}$ werden die Datenergänzung (Generierung von Einzelbaumdaten aus schichtenund artspezifischen Bestandesmittelwerten), die Z-Baum-basierte und zielstärkenorientierte Nutzungsschätzung (NAGEL 2004) und die Fortschreibung der Bestandesdaten gesteuert. Die Routinen zur Aktualisierung der Bestandesdaten nach Durchforstungen und Nutzungen werden ebenfalls von diesem Modul aufgerufen. Die Forsteinrichtungsdaten sind in den Tabellen einer Microsoft Access Datenbank gespeichert, auf die das Datensteuerungsmodul über die Datenbankschnittstelle DirectAccess ${ }^{13}$ zugreift.

Daten aus diesen Tabellen können mit einem speziellen Abfrage-Dialog selektiert werden, der neben der visuellen und komfortablen Erzeugung komplexer SQL-Abfragen auch deren Speicherung und Wiederverwendung, sowie den Export der selektierten Datensätze für die Verwendung in externen Programmen erlaubt. Die Inventurdaten werden entweder über die serielle oder USB-Schnittstelle direkt aus dem Speicher des MDE-Gerätes oder - wenn die Dateneingabe manuell erfolgte - aus einer ASCII-Datei eingelesen. Da die Auflösung der (gemessenen oder geschätzten) Daten bis zur Einzelbaumebene reicht, sind entsprechend differenzierte Abfragen möglich. In Verbindung mit den von SCHMIDT (2001) entwickelten Modellgleichungen, die eine kombinierte Stärke- und Güteklassensortierung auf der Ebene des Einzelbaumes erlauben, ist es z. B. möglich, abzuschätzen, wo und in und welchen Mengen ein bestimmtes Sortiment innerhalb des Forstbetriebes zu erwarten ist (vgl. a. SCHMIDT 2004). Entsprechende Kundenanfragen können auf diese Weise schnell beantwortet werden.

Ein zweites Programmodul erlaubt die kartographische Darstellung der interessierenden Bestände und die Visualisierung der Datenbankabfragen. Vor dem Hintergrund des räumlichen Bezugs der meisten Planungsprozesse und betrieblichen Maßnahmen ist der Nutzen Geographischer Informationssysteme (GIS) in der Forstwirtschaft unbestritten (z. B. BITTER 1990, SPORS et al. 1992, TeUfFEL und KREBS 1996). Aufgrund des räumlichen Charakters von Beständen und ihrer Bedeutung als Planungs-, Kontroll- und Buchungseinheit drängt sich die Verwendung eines GIS - natürlich besonders bei einem bestandesbezogenen Planungsansatz - geradezu auf. Das in Waldplaner+ integrierte GIS-Modul wurde mit Hilfe der MapObjects-Komponenten der Firma ESRI programmiert und ermöglicht den Zugriff auf die gängigsten Vektor- und Rasterdatenformate.

Das GIS-Modul dient als „Daten-Viewer“ v. a. der interaktiven und raumbezogenen Selektion von Beständen und Bestandesklassen und der Visualisierung von Abfragen und Auswertungen. Die erzeugten Kartenansichten können ausgedruckt oder in eine Grafikdatei gespeichert und in anderen Programmen weiterverwendet werden. Ein Beispiel wäre die Erzeugung einer Karte mit allen Beständen, deren Bestockungsgrad einen bestimmten kritischen Wert überschritten hat, um einen Überblick über die Anzahl, Gesamtfläche und Verteilung

\footnotetext{
12 http://treegross.sourceforge.net, 27. Juli 2007.

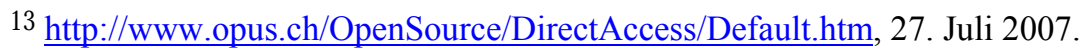


der besonders pflegebedürftigen Waldteile zu bekommen. Auf der Grundlage dieser Funktionalität kann der Waldplaner+ zu einem Werkzeug zur Unterstützung von Entscheidungsprozessen (Decision Support System) ausgebaut werden (vgl. KÄTSCH 1998; VACIK und LEXER 2001; SodTKE et al. 2005; NAGEL und SCHMIDT 2006). Gleichzeitig steht durch die Kompatibilität der verwendeten Datenformate die gesamte Funktionalität von Microsoft Access und der GIS-Programme ARC/INFO und ArcView zur Verfügung, so daß die Daten auf vielfältigste Art und Weise bearbeitet und analysiert werden können. 


\section{Diskussion}

Das hier vorgestellte Inventurkonzept und seine softwareseitige Umsetzung zielen darauf ab, dem Forstbetrieb möglichst exakt die Daten zur Verfügung zu stellen, die die operative Planung und Entscheidungsfindung bestmöglich unterstützen, um auf diese Weise zur Optimierung des Betriebsergebnisses beizutragen ${ }^{14}$. Da bei einer stratenorientierten Planung Informationen verlorengehen, die bezüglich dieser Entscheidungsprozesse für essentiell gehalten werden, wird im Gegensatz zum Konzept der Betriebsinventur am Bestand als primäre Bezugsebene für Inventur und Planung festgehalten ${ }^{15}$. Zur Reduktion der dadurch entstehenden Kosten beinhaltet das vorgestellte Konzept eine Reihe von Maßnahmen. Hierzu zählen insbesondere

- die Anpassung der Aufnahmeintensität (des Inventurverfahrens) an den tatsächlichen Informationsbedarf und an die vorhandenen Bestandesstrukturen,

- die Vergrößerung der durchschnittlichen Bestandesgröße auf mindestens 5-10 ha,

- die Fortschreibung des Datenbestandes auf der Basis von Wuchsmodellen und der erfaßten Nutzungsdaten und

- die dadurch ermöglichte Verlängerung des Inventurintervalls und Reduzierung der Kosten der mittelfristigen Betriebsplanung.

Zudem wird durch die Aktualität und den Ortsbezug der Daten deren Nutzwert deutlich erhöht. Dies betrifft insbesondere die Planung des Holzeinschlages und die Vermarktung des Rohholzes, die durch die Erfassung des ausscheidenden Bestandes erheblich effizienter und damit auch profitabler gestaltet werden können (z. B. NIEUWENHUIS et al. 2001). Da diese Datenerhebung nur einen überschaubaren Aufwand erfordert und gleichzeitig die Häufigkeit der Inventur des verbleibenden Bestandes reduziert, stellt sie eine besonders effiziente und damit unverzichtbare Komponente des Gesamtsystems dar.

Um den Nutzen aus diesem Inventursystem weiter zu steigern, sollten die Bestandesdaten in jedem Fall auch in Form eines geographischen Informationssystems (GIS) bereitgestellt werden. Eine weitere Effizienzsteigerung ist zu erwarten, wenn die Nutzung von Vorinformationen (z. B. aus großräumigen Waldinventuren) in das Berechnungssystem integriert wird

\footnotetext{
14 „Die operative Planung [...] dient auch zur laufenden Ausschöpfung und Anpassung der vorhandenen Leistungspotenziale. Dabei handelt es sich beispielsweise um spezielle Anpassungen des Produktionsprogramms mit dem Ziel, eine möglichst wirtschaftliche Nutzung der Ressourcen zu erreichen.“

(http://de.wikipedia.org/wiki/Operative_Planung, 26. Juli 2007)

15 Auch in Betrieben, die mit der Kontrollstichprobe inventarisiert werden, werden in aller Regel (allein schon aus der Notwendigkeit heraus, Betriebskarten anzufertigen) Bestände ausgeschieden, deren Vorräte und Nutzungen allerdings entweder mittels der Regionalisierung (s. Abschnitt 1) oder vom Forsteinrichter mit Hilfe der Vorinformationen aus der Betriebsinventur geschätzt werden (z. B. BöCKMANN et al. 1998b). Wie genau diese Schätzungen z. B. im Vergleich zu den Ergebnissen des konventionellen Waldbegangs sind und wie hoch dementsprechend ihr Nutzwert ist, ist jedoch unklar.
} 
(PuUmalainen 1998, S. 10 ff.). Das hier vorgestellte Datenmodell bildet aufgrund des bestandesbezogenen Ansatzes außerdem eine ideale Basis für eine auf der Mehrpfadtheorie beruhende Optimierung der gesamtbetrieblichen Planung (z. B. GADOW 2005, 2006).

Aufgrund der variierenden, vom Informationsbedürfnis der jeweils entscheidenden Personen abhängigen Inventurintervalle und -intensitäten liegt es nahe, die Verantwortung für die Erfassung der Bestandesdaten an die Forstbetriebsleitung zu delegieren. BULLINGER (2000) weist darauf hin, daß die Gesellschaft zur Zeit eine Umbruchphase der gesamten Arbeitswelt erlebt und diese Entwicklung besondere Anforderungen an die Arbeitsorganisation der Unternehmen stellt (vgl. a. KLOTZ 2000). Innovative Unternehmen werden sich demnach in Zukunft nicht nur durch innovative Produkte, sonders ebenso durch innovative Organisationsstrukturen auszeichnen. Als wesentliche Merkmale einer innovativen Organisation nennt er flache Hierarchien, dezentrale Entscheidungsstrukturen und eine ausgeprägte Ergebnisverantwortung der einzelnen Mitarbeiter und Arbeitsgruppen.

Wenn also der einzelne Forstbetrieb die Verantwortung für das Ergebnis seines Wirtschaftens tragen soll, sollte er auch die Instrumente und Mittel in die Hand bekommen, um diesen Erfolg steuern und messen zu können. Bezogen auf das Produktionsmittel und Warenlager „Wald“ bedeutet dies, daß dessen Zustand und Veränderung von denjenigen gemessen werden sollte, die auf diese Größen durch ihr Wirtschaften und ihre Entscheidungen direkten Einfluß nehmen. In diesem Sinne ist das Konzept der Eingriffsinventur als Instrument zur Selbstkontrolle zu verstehen, das insofern eine Alternative zur nachträglichen, externen Datenerfassung und Kontrolle durch die klassische Forsteinrichtung darstellt. Die Entscheidung, ob die Inventuren durch eigenes Personal oder durch forstliche Dienstleister durchzuführen sind, sollte ebenfalls dem einzelnen Forstbetrieb überlassen werden. Auch hier könnten mit der Einrichtung von evtl. auch forstbetriebsübergreifenden Cost-Centern neue Wege der Arbeitsorganisation beschritten werden (vgl. BULLINGER 2000).

Erste Erfahrungen mit dem hier vorgestellten Konzept in einem rund 1.800 ha großen Privatforstbetrieb zeigen, daß insbesondere die Erfassung der Nutzungen und deren „Verbuchung“ im dynamischen Betriebswerk sorgfältig in die Arbeitsabläufe integriert werden müssen, wenn sie die vorhandenen Arbeitskapazitäten nicht über Gebühr beanspruchen sollen. Dies erfordert auch eine entsprechende Schulung und Einweisung der Betriebsleitung und der mit dem Holzeinschlag beauftragten Mitarbeiter oder Unternehmer. So muß z. B. die korrekte Zuordnung der entnommenen Stämme zum Bestand gewährleistet sein, falls die Daten des ausscheidenden Bestandes durch den Harvester erfaßt werden. Hierbei ist die mit dem Waldplaner+ erstellte Arbeitskarte ein wichtiges Hilfsmittel, mittelfristig sollte jedoch der Einsatz von GIS- und GPS-gestützten Lösungen geprüft werden. In jedem Fall werden zusätzliche Aufgaben nur dann zuverlässig erfüllt werden, wenn ihr (Mehr-)Nutzen erkannt wurde. Dieser muß also auch kommuniziert werden, wodurch deutlich wird, daß innovative Organisati- 
onsstrukturen entsprechend hohe Anforderungen an Betriebsleitung, Mitarbeiter und Dienstleister stellen.

Die kritische Würdigung der Betriebsergebnisse und die mittelfristige Planung im Rahmen einer 10-jährigen Forsteinrichtung sollte weiterhin wesentlicher Bestandteil der gesamtbetrieblichen Planung und Kontrolle bleiben. Da durch die eingriffsbezogene Datenerfassung und Fortschreibung die aktuellen Zustandsdaten jedes Bestandes jederzeit verfügbar sind, kann hierbei auf messende Arbeiten vollständig verzichtet werden. Dies bedeutet eine erhebliche Einsparung von Mitteln, die freilich mindestens zum Teil den Forstbetrieben für die laufende Aktualisierung der Bestandesdaten zur Verfügung gestellt werden müßten. Darüberhinaus entstehen durch diese Entlastung von Inventuraufgaben Freiräume für eine Fokussierung der Forsteinrichtung auf die strategische Planung, wie sie u. a. von HANEWINKEL (2001) und BACHMANN et al. (2002) gefordert wird (vgl. a. BACHMANN 2005). Die Einbeziehung bestandesbezogener Informationen in den Prozeß der Gesamtplanung stellt hierzu keinen Widerspruch dar (vgl. GADOW 2005). Vielmehr erleichtert die Berücksichtigung der Gegebenheiten und Potentiale der zu bewirtschaftenden Einheiten - eben der Bestände - den Entwurf eines gesamtbetrieblichen Optimums, das operational ist und somit ein erreichbares Ziel darstellt. Die Methodik und technischen Möglichkeiten zur Umsetzung dieses Ansatzes stehen zur Verfügung. 


\section{Zusammenfassung}

In der vorliegenden Arbeit wird ein Konzept der permanenten Inventur vorgestellt, das den Bestand als Bezugsebene für Inventur und Planung in den Mittelpunkt stellt, da nur dadurch der Raumbezug erhalten bleibt, der für die Optimierung, Planung und Durchführung aller waldbaulichen Maßnahmen als essentiell erachtet wird. Unter Eingriffsinventuren werden in diesem Zusammenhang je nach Informationsbedarf in unterschiedlichen Zeitabständen stattfindende und flexibel gestaltete Inventuren zur Erfassung des Waldzustandes und dessen Veränderung verstanden. Wie gezeigt wird, eröffnet die damit verbundene bestandesindividuelle Anpassung sowohl der Inventurintervalle als auch der Inventurintensität in Verbindung mit einer Fortschreibung der Bestandesdaten ein beachtliches Rationalisierungspotential.

Der kontinuierlichen Erfassung der Nutzungen kommt dabei im Fortschreibungsprozeß eine besondere Bedeutung zu. Zugleich ist sie ein Instrument der betrieblichen Steuerung und dient, wenn sie vor dem Eingriff erfolgt, der Optimierung der Holzernte und einer kundenorientierten Rohholzbereitstellung. Im Sinne einer ausgeprägten Ergebnisverantwortung wird vorgeschlagen, die Verantwortung für die Erfassung der holzvorratsbezogenen Naturaldaten an die Forstbetriebsleitung zu delegieren. Das Konzept der Eingriffsinventur ist somit als Instrument zur Selbstkontrolle zu verstehen, das die periodisch stattfindende Forsteinrichtung weitestgehend von messenden Arbeiten entlastet und damit nicht nur weitere Kosteneinsparungen ermöglicht, sondern auch Freiräume für eine Konzentration auf die strategischen und gesamtbetrieblichen Aspekte der Forsteinrichtung schafft.

Innerhalb dieses Gesamtkonzepts bildet die Entwicklung von Methoden zur Erfassung des ausscheidenden und des verbleibenden Bestandes einen wesentlichen Schwerpunkt dieser Arbeit. Zur Erfassung des ausscheidenden Bestandes wird vorgeschlagen, in den Fällen, in denen die zu entnehmenden Bäume vor dem Eingriff ausgezeichnet werden, währenddessen die Art und den Brusthöhendurchmesser jedes markierten Baumes zu erfassen, was kaum zusätzlichen Zeitaufwand erfordert, wenn hierfür eine elektronische Kluppe eingesetzt wird. In Beständen, die vom Harvester durchforstet werden, könnte die Durchmesserverteilung alternativ aus den Daten des Bordcomputers generiert werden. Für alle anderen Fälle oder für jüngere Bestände, die zwar ausgezeichnet werden, in denen aber eine Vollkluppung aufgrund der hohen Stammzahlen nicht vertretbar ist, wird ein Verfahren vorgestellt, das die Durchmesserverteilung des ausscheidenden Bestandes auf der Basis der Weibullverteilung schätzt.

Auf der Datengrundlage von 67 Buchenreinbeständen aus neun Durchforstungsversuchen der Nordwestdeutschen Forstlichen Versuchsanstalt (B- bis E-Grad-Durchforstungen nach der Definition des Vereins ForstLICHER VerSuCHSANSTALTEN) wurden Regressionsgleichungen entwickelt, mit deren Hilfe die drei Parameter der Dichtefunktion der Weibullverteilung für einen gegebenen Bestand und bei gegebener Durchforstungsart prognostiziert werden können. Als Eingangsgrößen für dieses Modell werden der kleinster Durchmesser, der Mitteldurchmesser und die Stammzahl des Gesamtbestandes, sowie der Quotient aus den Ent- 
nahmequoten der Stammzahl und Grundfläche benötigt, wobei der letztgenannte Parameter die Durchforstungsart beschreibt. Wie anhand unabhängiger empirischer Daten und verschiedener Prüfgrößen gezeigt werden kann, erlaubt dieses Verfahren zuverlässige Schätzungen.

Für die Inventur des verbleibenden Bestandes werden unterschiedliche, in ihrer Genauigkeit und Informationsdichte den jeweiligen Bestandesverhältnissen und dem Bedarf angepaßte Verfahrensvarianten vorgestellt. Falls die Inventur mit Hilfe eines Stichprobenverfahrens erfolgen muß (aufgrund komplexer Strukturen und/oder Ungleichaltrigkeit) oder soll (bei besonderem Informationsbedarf hinsichtlich ökonomischer und/oder ökologischer Parameter), wird hierfür die 6-Baum-Stichprobe nach PRODAN vorgeschlagen, die als einfach und schnell anzuwendendes Verfahren der Bestandesinventur gilt. Da sie als Ein-Personen-Verfahren durchführbar ist, ist sie für die Anwendung im Rahmen von Eingriffsinventuren besonders geeignet. Ein Problem war bisher allerdings der nicht kalkulierbare, methodisch bedingte Bias bei der Schätzung der Stammzahl und Grundfläche pro Hektar in Beständen mit nichtzufälligem Baumverteilungsmuster. Wie aus der Literatur bekannt ist, ist der Bias der Stammzahlschätzung bei regelmäßiger räumlicher Verteilung der Bäume negativ und bei geklumpter Verteilung positiv, wobei sein Betrag mit zunehmender Abweichung von der Zufallsverteilung zunimmt.

Um den Zusammenhang zwischen räumlicher Verteilung und Bias der Stammzahl genauer quantifizieren zu können, wird mit dem punktbezogenen Winkelmaß $W_{P}$, basierend auf einem Ansatz von GADOW et al., ein Index vorgestellt, der im Zuge der 6-Baum-Stichprobe einfach und schnell bestimmt werden kann und eine relativ genaue Schätzung des Klumpungsgrades der Bäume erlaubt: Betrachtet man die von einem (Stichproben-)Punkt aus gesehen drei oder - im Fall der 6-Baum-Stichprobe - sechs nächsten Bäume und zählt die Winkel zwischen ihnen, die kleiner sind als ein vorher festgelegter kritischer Winkel $\alpha_{c}$, so beschreibt der Anteil dieser kleinen Winkel den Grad der Klumpung.

Es wird ein auf dem Winkelmaß beruhender Test vorgestellt, mit dem die Hypothese der vollständigen räumlichen Zufälligkeit (complete spatial randomness $=\mathrm{CSR}$ ) überprüft werden kann ( $W_{P}$-Test). Da die Mächtigkeit dieses Tests vom kritischen Winkel $\alpha_{c}$ abhängt, sollte dieser so gewählt werden, daß er die Teststärke bei gegebener Alternativhypothese maximiert. Es zeigte sich nämlich, daß die maximale Teststärke beim Test gegen die zwei möglichen Alternativhypothesen „Die Verteilung ist regelmäßiger als bei CSR“ und „die Verteilung ist geklumpter als bei CSR" mit unterschiedlichen $\alpha_{c}$ erreicht wird. Da aber in der praktischen Anwendung eine vorherige Einschätzung des Verteilungsmusters und damit eine Festlegung auf eine bestimmte Alternativhypothese i.d.R. nicht möglich ist, war also derjenige kritische Winkel zu finden, der hinsichtlich der Teststärke den bestmöglichen Kompromiß zwischen dem jeweils optimalen Wert für geklumpte und regelmäßige Verteilungen darstellt. Dieser Winkel wurde mit Hilfe von Monte Carlo Simulationen in künstlich erzeugten Baumpopulationen mit unterschiedlich regelmäßigen und geklumpten Verteilungen ermittelt. Im 
Fall des auf drei Bäumen beruhenden Winkelmaßes beträgt er $\alpha_{c}=60^{\circ}$, bei der Anwendung im Rahmen der 6-Baum-Stichprobe, d. h. bei Berücksichtigung der sechs nächsten Bäume, $\alpha_{c}=35^{\circ}$.

Basierend auf diesen Ergebnissen wurde in einem nächsten Schritt mit Hilfe von Stichprobensimulationen in ebenfalls künstlich erzeugten Punktmustern, die ein weites Spektrum von sehr regelmäßigen bis zu extrem geklumpten Verteilungen abdecken, untersucht, ob und wie der Bias der Stammzahlschätzung mit dem punktbezogenen Winkelmaß $W_{P}$ korreliert ist. Es zeigte sich, daß der $W_{P}$-Wert in einem sehr straffen, nichtlinearen Zusammenhang zum relativen Bias der Stammzahlschätzung steht, der mit Hilfe einer Regressionsgleichung, die über $97 \%$ der Streuung erklärt, sehr gut beschrieben werden kann. Dieses Modell wurde als bestandesindividueller Korrekturfaktor in den Schätzer für die Stammzahl pro ha integriert.

Zur Verminderung des Bias der Grundflächenschätzung und zur weiteren Verbesserung der Praktikabilität des Verfahrens werden zudem aufnahmetechnische Modifikationen vorgeschlagen: 1.) Die Beschränkung der eigentlichen 6-Baum-Stichprobe auf den Hauptbestand und die Erfassung des Nebenbestandes (soweit vorhanden) mittels okularer Schätzung von Mitteldurchmesser und Stammzahl innerhalb fester Probekreise (mit einem Radius von $6 \mathrm{~m}$ im Unterstand und $15 \mathrm{~m}$ im Überhalt), und 2.) die Beschränkung des Probekreisesradius der 6-Baum-Stichprobe auf maximal $15 \mathrm{~m}$. Die Validierung dieser modifizierten 6-BaumStichprobe mit Hilfe von Stichprobensimulationen zeigt, daß die vorgeschlagenen Änderungen den Betrag des Bias sowohl der Stammzahl-, als auch der Grundflächenschätzung selbst in sehr strukturreichen und heterogenen Beständen auf ein vertretbares Maß von unter $5 \%$ reduzieren und - in Verbindung mit ebenfalls reduzierten Standardfehlern - die Effizienz der 6-Baum-Stichprobe deutlich erhöhen. Dies ermöglicht ihre Anwendung in Beständen beliebiger Struktur.

Schließlich wird der Prototyp einer Software beschrieben, die als dynamisches Betriebswerk und Forstliches Informationssystem die Inventurdaten speichert, weiterverarbeitet und fortschreibt und sie in dieser aufbereiteten Form für die Entscheidungsträger zugänglich macht. Ein GIS-Modul, das geeignete digitale Rasterdaten (z. B. Orthophotos) und v. a. die Bestandesgeometrien vorhält, die wiederum über einen Schlüssel mit den Attributdaten der Forsteinrichtungsdatenbank verknüpft sind, wird als wesentlicher Bestandteil eines solchen Systems beschrieben. Über die Möglichkeit, komplexe Abfragen für beliebige Befundeinheiten bis auf die Einzelbaum-Ebene zu erstellen und die Ergebnisse sowohl in Form von aggregierten Übersichten und Tabellen, als auch in kartographischer Form auszugeben, wird es zum System zur Entscheidungsunterstützung, das - wie anhand weniger Beispiele gezeigt wird - ein erhebliches Potential birgt, über eine flexible Informationsbereitstellung bessere und schnellere Entscheidungen zu ermöglichen und damit zur Optimierung des Betriebsergebnisses beizutragen. 


\section{Literatur}

AKÇA, A., 1993: Zur Methodik und Bedeutung der kontinuierlichen Forstinventuren. Allg. Forst- u. Jagdztg.164 (11): 193 - 198.

AKÇA, A., 2001: Waldinventur. Cuvillier, Göttingen, 193 S.

AssunÇÃO, R., and REIS, I.A., 2000: Testing spatial randomness: A modification of the angle test. Brazilian Journal of Probability and Statistics: $71-86$.

BAChManN, P., 2005: Forstliche Planung - heute und morgen. Schweiz. Z. Forstwes. 156 (5): 137 - 141.

Bachmann, P., Ammann, S., Kunz, B., Rupf, A., 2002: Moderne forstbetriebliche Planung. Schweiz. Z. Forstwes. 153 (5): $184-189$.

BAdDeley, A., Turner, R., with contributions by Lieshout, M.-C. V., and 17 others, 2006: Spatstat: Spatial point pattern analysis, model-fitting and simulation. $\mathrm{R}$ package version 1.8-5, Internet: http://www.spatstat.org.

BEERS, T.W., 1966: The direct correction of boundery-line slopover in horizontal point sampling. Purdue Univ., Agri. Exp. Sta., Lafayette, Ind. Res. Progr. Rep. 224, 8 pp.

BIBER, P., 1997: Analyse verschiedener Strukturaspekte von Waldbeständen mit dem Wachstumssimulator SILVA 2. Vortrag anläßlich der Jahrestagung 1997 der Sektion Ertragskunde im Deutschen Verband Forstlicher Forschungsanstalten. Tagungsbericht: $100-120$.

BitTer, A.W, und Merrem, M., 1998: Typenorientierte Kontrollstichprobe. Konzept und Verfahrensweise. AFZ - Der Wald 52(23): 1414 - 1417.

BITTER, A.W., 1990: EDV-gestützte Unternehmensführung im Forstbetrieb mit Hilfe eines flächenbezogenen Betriebsinformationssystems. Diss. Forstl. Fak., Univ. Göttingen.

Blust, M., Korn-Allan, E., und VON Der Goltz, H., 2006: Nachhaltigkeitsmonitoring mittels Betriebsinventur. AFZ/Der Wald 61 (9): 469 - 470.

Böckmann, Th., Saborowski, J., Dahm, S. Nagel, J., und SpellmanN, H., 1998a: Die Weiterentwicklung der Betriebsinventur in Niedersachsen. Forst u. Holz 53 (8): 219 - 226.

Böckmann, Th., Spellmann, H. und HÜsing, F., 1998b: Neukonzeption und Weiterentwicklung der Forsteinrichtung in den Niedersächsischen Landesforsten. Forst u. Holz 53 (10): 298 - 302.

Bullinger, H.J., 2000: Arbeitsorganisation im Umbruch - Chancen für Mitarbeiter und Betriebe. Forst u. Holz 55: $534-540$.

Diggle, P.J., Besag, J.E., and Glaves, J.T., 1976: Statistical analysis of spatial point patterns by means of distance methods. Biometrics 32: $659-667$.

FÄHSER, L., 1983: Dynamisierung der Forsteinrichtung. AFZ - Der Wald 38 (17): 430 - 432.

FuchS, A., und KENNEL, E., 1994: Erste Wiederholungsaufnahme einer permanenten Betriebsinventur im bayerischen Staatswald. Forstw. Cbl. 113: 224 - 235.

Gadow, K. V., 1999: Waldstruktur und Diversität. Allg. Forst- u. Jagdztg. 170 (7): 117 - 122.

GADOW, K. v., 2005: Das Mehrpfad-Prinzip der Forsteinrichtung. AFZ - Der Wald 60 (5): 469 - 471.

GADOw, K. V., 2006: Forsteinrichtung - Adaptive Steuerung und Mehrpfadprinzip. Universitätsverlag Göttingen, $163 \mathrm{~S}$.

GADOw, K. V., und CHEN, B.W., 2002: Timber harvest planning with spatial objectives, using the method of simulated annealing. Forstw. Cbl. 121: $25-34$.

GAdow, K. V., und PuUmalainen, J., 2000: Scenario planning for sustainable forest management. In: GADOw, K. v., PukKalA, T., and Tomé, M., 2000: Sustainable Forest Management. Kluwer Series Managing Forest Ecosystems, Vol. 1: 319 - 356.

GADOw, K. V., und SCHMIDT, M., 1998: Periodische Inventuren und Eingriffsinventuren. Forst u. Holz 49 (5):129 - 131. 
Gadow, K. V., und StÜBER, V., 1994: Die Inventuren der Forsteinrichtung. Forst u. Holz 49 (5): 129 - 131.

GADOw, K. v., HuI, G.Y., und AlBERT, M., 1998: Das Winkelmaß - ein Strukturparameter zur Beschreibung der Individualverteilung in Waldbeständen. Centralbl. f. d. ges. Forstw. 115: 1 - 10.

GAFFrey, D., SABOROwSKi, J., und Spelsberg, G., 1998: Baumartenspezifische Funktionen zur Schätzung von Bestandesdurchmesserverteilungen. Allg. Forst- u. Jagdztg. 169 (5): 81 - 86.

GARCIA, O., 1981: Simplified method-of-moments estimation for the weibull distribution. New Zealand Jour. For. Sci. (11) 3: 304 - 305.

Gleichmar, W., und Gerold, D., 1998: Indizes zur Charakterisierung der horizontalen Baumverteilung. Forstwiss. Centralbl. 117: $69-80$.

GREgorius, H.-R., 1974: Genetischer Abstand zwischen Populationen - Zur Konzeption der genetischen Abstandsmessung. Silvae Genetica 23: 22-27.

GÜSSEFELDT, J., 1997: Grundsätzliche Überlegungen zu Regionalisierungsmodellen. Geograph. Zeitschr. 85: $1-19$.

Hallenbarter, D., Hasenauer, H., und ZINGG, A., 2005: Validierung des Waldwachstumsmodells MOSES für Schweizer Wälder. Schweiz. Z. Forstwes. 156 (5) : 149 - 156.

HANEWINKEL, M., 2001 : Neuausrichtung der Forsteinrichtung als strategisches Managementinstrument. Allg. Forst- u. Jagdztg. 172: 202 - 211.

Hasenauer, H., Moser, M., und EcKmÜLlner, O., 1995: Ein Programm zur Modellierung von Wachstumsreaktionen. AFZ - Der Wald 50 (4): 216 - 218.

HeInIMANN, H.R., 1999: Logistik der Holzproduktion - Stand und Entwicklungsperspektiven. Forstwiss. Centralbl. 118 (1): 24 - 38.

HessenMÖLlER, D., und GADOw, K. v., 2001: Beschreibung der Durchmesserverteilung von Buchenbeständen mit Hilfe der bimodalen Weibullfunktion. Allg. Forst- u. Jagdztg. 172 (3): 46 - 50.

HinRichs, A., Oesten, G., und SteinBrenner, M., 1993: Die Betriebsinventur der Landesforstverwaltung Baden-Württemberg. Arbeitspapier 14-93. Institut für Forsteinrichtung und Forstliche Betriebswirtschaft, Albert-Ludwigs-Univ. Freiburg, $74 \mathrm{~S}$.

HIRNER, V., 1978: Theoretische Überlegungen zur Sechs-Baum-Stichprobe und deren praktische Anwendungsmöglichkeiten. Univ. Freiburg (Breisgau), Forstwiss. Fak., Diss., 114 S.

HOCKENJOS, W., 2006: Naturschutzstandards durch Zertifizierungssysteme - Zurück zum Holzacker? AFZ - Der Wald 61 (3): 146 - 151.

Hostettler, M., 2006: Bewirtschaftung des Staatswaldes in einer globalen Wirtschaft. Forst u. Holz 61 (7), $270-273$.

Horwitz, D., and Thompson, D., 1952: A Generalisation of Sampling without Replacement from a finite Universe“, J. Amer. Stat. Ass. 47: 663 - 685.

JonsSON, B., Holm, S., and KALluR, H., 1992: A forest inventory method based on density-adapted circular plot size. Scand. J. For. Res. 7: $405-421$.

KASSIER, H.W., 1993: Dynamics of diameter and height distributions in commercial timber plantations. PhD dissertation, Faculty of Forestry, Univ. of Stellenbosch, South Africa.

KÄTsCH, C., 1998: Konzeption für ein forstliches Produktionsplanungs- und Steuerungssystem unter besonderer Berücksichtigung der Forsteinrichtung. Schriften aus der Forstl. Fak. der Univ. Göttingen und der Nieders. Forstl. Versuchsanstalt 126. Sauerländer's, Frankfurt a. Main, 211 S.

KIMMINS, J.P., 1997: Forest ecology - a foundation for sustainable management, $2^{\text {nd }}$ Ed., Prentice Hall, Upper Saddle River, NJ, 596 pp.

KLEINN, CH., und VILČKO, F., 2005: Ein Vergleich von zwei methodischen Konzepten für die Grundgesamtheit von Probeflächen bei Waldinventuren. Allg. Forst- u. Jagdztg. 176: 68 - 74.

KLEINN, CH., and VILČKO, F., 2006: Design unbiased estimation for point-to-tree distance sampling. Can. J. For. Res. 36 (6): 1407 - 1414. 
KLotZ, U., 2000: Neue Unternehmensmodelle führen zu einer anderen Definition von Arbeit. Neue Ökonomie, Teil 3. Frankf. Allg. Zeitung 124, S. 33.

KöHL, M., und ZINGG, A., 1995: Eignung von Diversitätsindizes bei Langzeituntersuchungen zur Biodiversität in Waldbeständen. Allg. Forst- u. Jagdztg. 167 (4): $76-85$.

Kramer, H., 1990: Nutzungsplanung in der Forsteinrichtung. 2. Aufl., Sauerländer, Frankfurt a.M., 157 S.

Kramer, H., und AKÇA, A., 1995: Leitfaden zur Waldmeßlehre. 3. Aufl., J. D. Sauerländer's, Frankfurt a. M., $266 \mathrm{~S}$.

LeSSARD, V., Drummer, T.D., and Reed, D.D., 2002: Precision of Density Estimates from Fixed-Radius Plots Compared to N-Tree Distance Sampling. Forest Science 48 (1): $1-6$.

LewANDOWSKI, A., und GADOW, K. V., 1997: Ein heuristischer Ansatz zur Reproduktion von Waldbeständen. Allg. Forst- u. Jagdztg. 168 (9): $170-174$.

MCPFE, 2000: General Declarations and Resolutions adopted at the Ministerial Conference on the Protection of the Forests in Europe, Strasbourg 1990 - Helsinki 1993 - Lisbon 1998. Liaison Unit Vienna, 88 p.

MCPFE, 2003: Improved Pan-European indicators for sustainable forest management as adopted by the MCPFE Expert Level Meeting 7-8 October 2002, Vienna, Austria. Vienna, Austria, MCPFE Liaison Unit. Available online at: http://www.mcpfe.org/system/files/u1/publications/pdf/improved_indicators.pdf.

MERKER, K., 2006: Bewirtschaftung des Staatswaldes in einer globalen Wirtschaft. Forst u. Holz 61 (7): $250-252$.

Muinonen, E., Matama, M., Hyppänen, H, and Vainikainen, V., 2001: Forest stand charachteristics estimation using a most similar neigbour approach and image spatial structure information. Remote Sensing of nvironment 78: 223 -228.

NAGEL, J., 1988: Volumenfunktionen. Interner Bericht, Nordwestdeutsche Forstliche Versuchsanstalt, Göttingen.

NAGEL, J., 1998: Zur Auswertung und Zuwachsprognose von permanenten Stichprobenerhebungen. Forst u. Holz 53 (7): 197 - 198.

NAGEL, J., 1999: Konzeptionelle Überlegungen zum schrittweisen Aufbau eines waldwachstumskundlichen Simulationssystems für Nordwestdeutschland. Schriften aus der Forstl. Fak. d. Univ. Göttingen und der Nieders. Forstl. Versuchsanst. 128, J. D. Sauerländer's, Frankf. a. M., 122 S.

NAGEL, J., 2000: Prognose des Zuwachses von Fichten/Buchen-Mischbeständen mit dem Programm BWIN. In: Saborowski, J., und Sloboda, B. (Hrsg.): Deutscher Verband Forstlicher Forschungsanstalten, Sektion Forstliche Biometrie und Informatik - 12. Jahrestagung und Internationale Biometrische Gesellschaft Deutsche Region, Arbeitsgruppe Ökologie, Herbstkolloquium, Göttingen, 29. September - 1. Oktober 1999; Die grüne Reihe: 194 - 199.

NAGEL, J., 2004: Nutzungsplanung in Rein- und Mischbeständen. Allg. Forst- u. Jagdztg. 175 (7/8): 150 - 156.

NAGEL, J., 2005: TreeGrOSS - Eine Java basierte Softwarekomponente zur Waldwachstumsmodellierung für Forschung, Lehre und Praxis. In: Wunn, U., und Quedenau, H.D. (Hrsg.): Deutscher Verband Forstlicher Forschungsanstalten Sektion Forstliche Biometrie und Informatik, 15. Tagung Freiburg 9.-10. Oktober 2003, Grüne Reihe: 33 - 37

NAGel, J., und Biging, G.S., 1995: Schätzung der Parameter der Weibullfunktion zur Generierung von Durchmesserverteilungen. Allg. Forst- u. Jagdztg.166 (9/10): 185 - 189.

NAGel, J., and Schmidt, M., 2006: The Silvicultural Decision Support System BWINPro. In: HaSenauer, H. (ed.): Sustainable Forest Management, Growth Models For Europe, Springer, Berlin, Heidelberg: 59 63.

Nicke, A., ERTeld, TH., KÖHLER, B., und TAUTKUS, M., 2005: Inventur in strukturreichen Beständen. AFZ Der Wald 60 (5): $245-247$.

NIEDERSÄCHSISCHE LANDESFORSTEN,1987: Anweisung zur Betriebsregelung (Forsteinrichtung) in den Niedersächsischen Landesforsten. Nieders. Minist. f. Ernähr., Landwirtsch. u. Forsten, 55 S. 
Nieschulze, J., Böckmann, Th., NAgel, J., und SABorowski, J., 2005: Herleitung von einzelbestandesweisen Informationen aus Betriebsinventuren für die Zwecke der Forsteinrichtung. Allg. Forst- u. Jagdztg. 176 (9/10): 169 - 176.

Nieuwenhuis, M., McHugh, F., Malone, L., and Layton, T, 2001: Development and evaluation of pre-harvest inventory and cross-cutting simulation procedures to maximise value recovery. Irish Forestry 56 (1): $12-28$.

Отто, H.-J., 1992: Langfristige ökologische Waldentwicklung: Ökologische Grundlagen des Regierungsprogramms. AFZ 47 (11): $566-568$.

OтTо, H.-J., 1994: Waldökologie. Ulmer, Stuttgart, 391 S.

PiCARD, N., KouYaté, A.M., and Dessard, H., 2005: Tree density estimation using a distance method in Mali Savanna. For. Sci. 51 (1): $7-18$.

Pommerening, A., 1997: Eine Analyse neuer Ansätze zur Bestandesinventur in strukturreichen Wäldern. Göttingen, Univ., Diss., $150 \mathrm{~S}$.

POMMERENING, A., 1998: Fortschreibung von Stichprobendaten mit positionsabhängigen Wuchsmodellen. Vortrag anläßlich der Jahrestagung 1998 der Sektion Ertragskunde des Deutschen Verbandes Forstlicher Forschungsanstalten, Tagungsband: $35-51$.

Pommerening, A., 2006: Evaluating structural indices by reversing forest structural analysis. Forest Ecology and Management 224: 266 - 277.

Pommerening, A., und Gadow, K. V., 2000: Zu den Möglichkeiten und Grenzen der Strukturerfassung mit Waldinventuren. Forst und Holz 55(19): $622-631$.

Pommerening, A., und SCHMIDT, M., 1998: Modifizierung des Stammabstandsverfahrens zur Verbesserung der Stammzahl- und Grundflächenschätzung. Forstarchiv 69: 47 - 53.

Pretzsch, H., 1995: Zum Einfluß des Baumverteilungsmusters auf den Bestandeszuwachs. Allg. Forst- u. Jagdztg. 166: $190-201$.

PreTZSCH, H., 1997: Analysis and modeling of spatial stand structures. Methological considerations based on mixed beech-larch stands in Lower Saxony. Forest Ecology and Management. Vol. 97: 237 - 253.

PretZSCH, H., und DURSKY, J., 2001: Evaluierung von Waldwachstumssimulatoren auf Baum- und Bestandesebene. Allg. Forst- u. Jagdztg. 172 (8/9): $146-150$.

PRETZSCH, H., und KAHN, M., 1996: Wuchsmodelle für die Unterstützung der Wirtschaftsplanung im Forstbetrieb. AFZ - Der Wald 51 (25): 1414 - 1419.

Prodan, M., 1968: Punktstichprobe für die Forsteinrichtung. Der Forst- u. Holzwirt 23: 225 - 226.

Programmleitung holz 21 (Hrsg.), 2003: Erfolgsfaktoren für die Wald- und Holzwirtschaft - Ergebnisse einer richtungweisenden Studie. holz 21, ein Förderprogramm des Bundesamtes für Umwelt BAFU, www.holz21.ch.

Puumalainen, J., 1998: Marktorientierte Vorratschätzung und terrestrische Vorinformationen in kleinräumigen Waldinventuren. Diss., Fak. f. Forstwissensch., Univ. Göttingen, Cuvillier Verlag Göttingen, 176 S.

Puumalainen, J., Gadow, K. v, und Korhonen, K., 1998: Flexible Ergebnisberechnung einer Eingriffsinventur. Forstarchiv 69: $145-150$.

R DeVelopment CoRe TEAm, 2005: R: A language and environment for statistical computing. R Foundation for Statistical Computing, Vienna, Austria. ISBN 3-900051-07-0, Internet: http://www.R-project.org.

RHEINLAND-PFÄLZISCHES ForSTAMT DAUn, 2000: Aufnahme des ausscheidenden Bestandes mit verfahrensbezogener Sorten-, Kosten- und Ergebnisschätzung. AFZ - Der Wald 55 (18-19): 997 - 998.

SABOROWSKI, J. und ŠMELKO, S., 1998: Zur Auswertung von Stichprobeninventuren mit variablen Probeflächengrößen. Allg. Forst- u. Jagdztg. 169 (4): 71 - 75.

SAGL, W., 1995: Forsteinrichtung auf dem Prüfstand. Schriftenr. Inst. forstl. Betriebswirtsch. u. Forstwirtschaftspolitik d. Univ. f. Bodenkultur Wien Nr. 26, 137 S. 
Schmid-HAAS, P., 1969a: Kontroll-Stichproben: Aufnahmeinstruktion. Mitt. der Eidgen. Anstalt. Forstl. Vers. 27.

Schmid-HAAS, P., 1969b: Stichproben am Waldrand. Mitt. der Eidgen. Anstalt. Forstl. Vers. 45: 233 - 303.

SchMID-HAAS, P., 1989: Schweizer Kontrollstichprobenverfahren in der Forsteinrichtung. Schweiz. Z. Forstwes. 140: 43 - 56.

SCHMIDT, M., 2001: Prognosemodelle für ausgewählte Holzqualitätsmerkmale wichtiger Baumarten. Diss., Fak. F. Forstwiss., Univ. Göttingen, $302 \mathrm{~S}$.

SCHMIDT, M., 2004: Simulative Astigkeits- und Qualitätsprognose für Douglasien-Rundholz. Allg. Forst- u. Jagdzeitg. 175: $49-60$.

SCHOBER, R., 1995: Ertragstafeln wichtiger Baumarten. 4. Aufl., Sauerländer, Frankfurt a. M.

SCHÖNSLEBEN, P., 2000: Integrales Logistikmanagement : Planung und Steuerung von umfassenden Geschäftsprozessen. 2., überarb. und erw. Aufl., Springer, Berlin, 778 S.

SCHÖPFER, W., 1998: Schritte zu einem Informationssystem für Holzernte und Holzvermarktung. Forst und Holz 53 (12): $379-387$.

SCHÜTZ, J.-PH., 2002: Die Plenterung und ihre unterschiedlichen Formen - Skript zu Vorlesung Waldbau II und Waldbau IV. Professur Waldbau, ETH Zürich, $126 \mathrm{~S}$.

SMALTSCHINSKI, TH., 1998: Charakterisierung von Baumverteilungen. Forstwiss. Centralbl. 117: 355 - 361.

Sodtke, R., Schmidt, M., Fabrika, M., Nagel, J., Dursky, J., und Pretzsch, H., 2004 : Anwendung und Einsatz von Einzelbaummodellen als Komponenten von entscheidungsunterstützenden Systemen für die strategische Forstbetriebsplanung. Forstarchiv (75) 51-64.

SPEIDEL, G., 1972: Planung im Forstbetrieb. Parey, Hamburg und Berlin, 276 S.

SpellmanN, H., 1995: Vom strukturarmen zum strukturreichen Wald. Forst u. Holz 50 (2): 35 - 44.

Spellmann, H., und AKৎ̧A, A., 1983: Verbesserungsmöglichkeiten in der Forsteinrichtung. AFZ - Der Wald 38 (17): 427 - 430.

Spellmann, H., Nagel, J., und BöCKMAnN, TH., 1999: Summarische Nutzungsplanung auf der Basis von Betriebsinventuren. Allg. Forst- u. Jagdztg.170 (7): $122-128$.

SpORS, H.-J., STOCK, R., und SlOBODA, B., 1992: Räumliche Informationssysteme als Entscheidungsbasis für die forstliche Praxis. Forstarchiv 63: 33 - 39.

STAUPENDAHL, K., 1997: Ein neues Stichprobenverfahren zur Erfassung und Beschreibung von Naturverjüngung. In: Pelz, D.R. (Hrsg.): Deutscher Verband Forstlicher Forschungsanstalten, Sektion Forstliche Biometrie und Informatik - 10. Jahrestagung, Freiburg, 24. - 26. September 1997, IUFRO, Grüne Reihe: $32-49$.

STAUPENDAHL, K., 1999: Modellierung von Durchforstungen in Buchenreinbeständen auf der Basis des Stammzahl-Grundflächen-Verhältnisses. In: Kenk, G. (Hrsg.), 1999: Deutscher Verband Forstlicher Forschungsanstalten, Sektion Ertragskunde - Beiträge zur Jahrestagung in Volpriehausen (Solling), 17.-19.5.1999: 112 - 125 .

Staupendahl, K., 2001: PoiSSim - Ein Programm zur Simulation von Punktstichproben. ARGUS Forstplanung, Göttingen.

Staupendahl, K., und PuUmalainen, J., 1999: Modellierung des Einflusses von Durchforstungen auf die Durchmesserverteilung von gleichaltrigen Fichtenreinbeständen. Centralbl. f. d. ges. Forstw. 116 (4): $249-262$.

STERBA, H., 1990: Validitätsprüfung des Einzelbaumsimulators für Fichten-Kiefern-Mischbestände. Tagungsbericht, Deutscher Verb. Forstl. Forschungsanstalten - Sektion Ertragskunde: 101 - 117.

STOYAN, D., 1995: Stochastic Geometry 4.1 - ein Windows-Programm zur Erzeugung, Darstellung und statistischen Auswertung verschiedener Modelle der ebenen Stochastischen Geometrie.

Stoyan, D., Und Stoyan, H., 1992: Fraktale, Formen, Punktfelder: Methoden der Geometrie-Statistik. Berlin, Akad.-Verl., $394 \mathrm{~S}$. 
STRAUSS, D.J., 1975: A model for clustering. Biometrika 62: 467 - 475.

STÜMER, W., 2004: Kombination von terrestrischen Aufnahmen und Fernerkundungsdaten mit Hilfe der kNNMethode zur Klassifizierung und Kartierung von Wäldern. Diss., Fak. Forst-, Geo- und Hydrowiss., Techn. Univ. Dresden, 153 S.

SzAro, R.C., and Johnston, D.W., 1996: Biodiversity in managed landscapes. Oxford University Press, 778 pp.

Teuffel, K. Frhr. V., und KreBS, M. 1996: Geoinformationssysteme als Instrumente der forstlichen Betriebsführung. AFZ - Der Wald 10: 532 - 542.

Teuffel, K. Frhr. V., und Krebs, M., 1999: Forsteinrichtung im Wandel. AFZ - Der Wald 54: 858 - 865.

TzschupKE, W., 1991: Betriebs- oder bestandesbezogene Waldzustandsinventuren für die Forsteinrichtung. AFZ - Der Wald162 (10): 195 - 200.

Upton, G., and Fingleton, B., 1989: Spatial Data Analysis by Example. Vol. 2: Categorical and Directional Data. John Wiley \& Sons, Chichester, 416 pp.

Upton, G., and Fingleton, B., 1990: Spatial Data Analysis by Example. Vol. 1: Point Pattern and Quantitative Data. $2^{\text {nd }}$ Ed., John Wiley \& Sons, Chichester, 410 pp.

Uusitalo, J., 1995: Pre-harvest measurement of pine stands for sawing production planning. University of Helsinki, Department of Forest Resource Management, Publications 9, 96 p.

UUsitalo, J., and KIVINEN, V.P., 2000: EMO: A pre-harvest measurement tool for predicting forest composition. In: Usenius, A. et al. (Eds.): Proc. of 3rd workshop on measuring of wood properties, grades and qualities in the conversion chains and global wood chain optimisation in Espoo, Finland: 173 - 180.

VACIK, H, and LEXER, M., 2001: Application of a spatial decision support system in managing the protection forests of Vienna for sustained yield of water resources. For. Ecol. Managem. 128: 1 -2, 49 - 55.

VANClAY, J., 1998: Towards more rigorous assessment of biodiversity. In: BACHMANN, P., KöHL, M., and PÄIVINEN, R. (eds.): Assessment of biodiversity for improved forest planning. Kluwer Academic Publishers: $211-232$.

Venables, W.N., and RIPLEY, B.D., 2002: Modern Applied Statistics with S. Fourth Edition. Springer, New York, $495 \mathrm{pp}$.

Verein ForSTLICHER VeRSuChSANSTALTEN, 1902: Beratungen der vom Verein Forstlicher Versuchsanstalten eingesetzten Kommission zur Feststellung des neuen Arbeitsplanes für Durchforstungs- und Lichtungsversuche. Allg. Forst- u. Jagdztg. 78: 180 - 184.

WAlter, F., and CARLSSOn, D., 1998: New system for optimizing transport. SkogForsk News 1: p. 1.

WeIDENBACH, P., und KARIUS, K., 1993: Betriebsinventur auf Stichprobenbasis als Element moderner Forsteinrichtung. AFZ - Der Wald 48 (13): 685 - 688. 



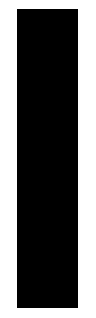





\title{
Eingriffsinventuren und dynamisches Betriebswerk - Instrumente der operativen Planung im Forstbetrieb
}

Thinning event assessment and dynamic management plan tools for operational planning in the forest enterprise

\author{
Kai StaUPENDAHL ${ }^{1}$ und KLAUS V. GADOW ${ }^{2}$
}

Forstarchiv 79 (im Druck)

Schlagwörter: Bestandesinventur, Fortschreibung, Forsteinrichtung, Informationssystem, Entscheidungsunterstützungssystem.

\section{Zusammenfassung}

In der vorliegenden Arbeit wird ein Konzept der permanenten Inventur vorgestellt, das den Bestand als Bezugsebene für Inventur und Planung in den Mittelpunkt stellt, da nur dadurch der Raumbezug erhalten bleibt, der für eine optimierte Planung und Durchführung aller waldbaulichen Maßnahmen als essentiell erachtet wird. Die bestandesindividuelle Anpassung sowohl der Inventurintervalle als auch der Inventurintensität eröffnet dabei in Verbindung mit einer Fortschreibung der Bestandesdaten ein beachtliches Rationalisierungspotential. Der kontinuierlichen Erfassung der Nutzungen (Eingriffsinventur) kommt dabei im Fortschreibungsprozeß eine besondere Bedeutung zu. Zugleich ist sie ein Instrument der betrieblichen Steuerung und dient, wenn sie während oder nach dem Auszeichnen und vor dem Eingriff erfolgt, der Optimierung der Holzernte und einer kundenorientierten Rohholzbereitstellung. Die Verantwortung für die Erfassung der holzvorratsbezogenen Naturaldaten sollte im Sinne einer ausgeprägten Ergebnisverantwortung an die Forstbetriebsleitung delegiert werden. In diesem Sinne ist das Konzept der Eingriffsinventur als Instrument zur Selbstkontrolle zu verstehen, das die periodisch stattfindende Forsteinrichtung weitestgehend von messenden Arbeiten entlastet und ihr damit eine Konzentration auf die strategischen, gesamtbetrieblichen Kontroll- und Planungsarbeiten erlaubt.

Key words: Stand inventory, projection, forest management, information system, decision support system.

\footnotetext{
1 Dipl.-Forstw. Kai Staupendahl (Kontaktadresse), ARGUS Forstplanung, Büsgenweg 5, D-37077 Göttingen, Telefon: +49-(0)551 3934 76, Fax: +49 (0)551 3957 67, E-Mail: staupendahl@argus-forstplanung.de.

2 Prof. Dr. Klaus v. Gadow, Institut für Waldinventur und Waldwachstum, Georg-August-Universität Göttingen, Büsgenweg 5, D-37077 Göttingen, Tel.: +49 (0)551 3934 72, Fax: +49 (0)551 3997 87, E-Mail: kgadow@gwdg.de.
} 


\begin{abstract}
This paper presents the general concept and specific application details of a permanent forest inventory in which the forest stand is the reference unit for resource assessment and planning. The stand-oriented approach provides spatially explicit information which is essential for optimizing silviculture and for timing harvesting operations. It differs in principle from the region-oriented approach which provides information about the forest resource on the landscape or property level. Considerable savings of time and effort may be achieved if the time intervals between successive inventories and the intensity of the assessments are adapted to the stand-specific information needs. Such stand-specific adaptation strategies are presented in this paper. Especially important is the continuous assessment of the harvest, known as "thinning event assessment". A thinning event assessment can greatly increase the effectiveness of harvesting operations and improve the information flow from forest stand to client. The responsibility for the field assessment should be delegated to the local management staff who can appreciate the importance of accurate resource data. In this sense, the concept of a permanent forest inventory can be understood as an effective mechanism for self-control which will considerably reduce the measurement effort normally required in a periodic forest inventory. This will allow planning staff to concentrate on other important tasks, such as monitoring and planning at enterprise level.
\end{abstract}

\title{
1 Problemstellung
}

Grundlage einer auf Nachhaltigkeit bedachten Forstplanung sind verläßliche Informationen über Holzvorräte, Wert und Struktur des bewirtschafteten Waldes. In den bisher vorherrschenden Altersklassenwäldern wurden diese Daten mit Hilfe periodischer Bestandesinventuren ermittelt, wobei der sogenannte Waldbegang mit Ertragstafelschätzung das überwiegend angewandte Verfahren war und teilweise noch ist (KRAMER und AKÇA 1995, S. 163 f.). Die in den letzten Jahren bis Jahrzehnten auf großer Fläche praktizierte Zielstärkennutzung mit verlängerten Verjüngungszeiträumen (z. В. Отто 1992) hat im Zusammenwirken mit veränderten Wachstumsbedingungen und Durchforstungskonzepten dazu geführt, daß das Ertragstafelkonzept auf großer Fläche nicht mehr sinnvoll anwendbar ist. Bedingt durch höhere Artenvielfalt und Ungleichaltrigkeit entstehen in naturnah bewirtschafteten Forstbetrieben immer komplexere Waldstrukturen. Dadurch hat die Betriebsinventur (Kontrollstichprobe) als alternatives Inventurkonzept zunehmend an Bedeutung gewonnen (SCHMID-HAAS 1969a, 1989; WeIDENBACH und KARIUS 1993; AKÇA 1993).

Beispiele für Betriebsinventuren sind die Verfahren der Landesforstbetriebe bzw. -verwaltungen Niedersachsen (BöCKMANN et al. 1998a, 1998b), Bayern (FUCHS und KENNEL 1994) und Baden-Württemberg (HINRICHS et al. 1993; TEUFFEL und KREBS 1999). Sie ermög- 
lichen die Schätzung von metrischen Variablen für sogenannte Befundeinheiten bzw. Straten. Die Vorteile der Betriebsinventuren liegen insbesondere in der Objektivierung der Erfassung des Waldzustandes, einer statistisch abgesicherten Beschreibung der mittel- bis langfristigen Waldentwicklung (bei vorliegenden Wiederholungsinventuren) und relativ flexiblen Auswertungsmöglichkeiten innerhalb der Straten und auf der Ebene des Gesamtbetriebes.

Diesen Vorteilen stehen jedoch einige Nachteile gegenüber (GADOW und SCHMIDT 1998). So lassen sich die Daten aus Betriebsinventuren z. B. kaum für die operative Planung nutzen, was u. a. daran liegt, daß keine bestandesbezogenen Daten erhoben werden, da ein Bestand i.d.R. zu wenig (oder gar keine) Probekreise enthält und somit die in den Probekreisen enthaltenen Informationen nicht ohne weiteres auf die Bestandesebene übertragen werden können. Zur Lösung dieses Problems der Übertragung von punktbezogenen Informationen in die Fläche (Regionalisierung) sind mittlerweile einige Ansätze vorgestellt worden. Sie reichen von der einfachen gutachtlichen Schätzung (z. B. BÖCKMANN et al. 1998b) bis zu Methoden der räumlichen Statistik (vgl. GÜSSEFELDT 1997), wobei dem Einsatz von Luftbildern und Satellitendaten eine besondere Bedeutung zukommt (z. B. MUINONEN et al. 2001; STÜMER 2004; NIESCHULZE et al. 2005). Die Ergebnisse sind allerdings nur teilweise zufriedenstellend und erfordern natürlich zusätzlichen Mitteleinsatz. Inwieweit dabei der Nutzen in einem günstigen Verhältnis zu den Kosten steht, wurde bisher nicht untersucht.

Wenn die Regionalisierung der Probekreisdaten nur unvollständig möglich ist, erhält man also genaue Informationen über die Holzvorräte, aber nur sehr ungenaue über deren Örtlichkeit. Dieser Raumbezug, der für die Optimierung, Planung und Durchführung aller waldbaulichen Maßnahmen entscheidend ist, kann nur über den Bestand als topologisch eindeutig definierte Buchungs- und Kontrolleinheit gewährleistet werden. Seine Bedeutung kommt auch in der Anwendung geographischer Informationssysteme zum Ausdruck, die zunehmend zur Optimierung der Holzernte und Transportprozesse genutzt werden (z. B. GADOW und CHEN 2002; WALTER und CARLSSON 1998).

Da mit der Betriebsinventur eine ausreichend genaue Zustandsbeschreibung auf Bestandesebene nur selten möglich ist, werden natürlich auch die Durchforstungen und Erntemaßnahmen nur für die einzelnen Straten geplant, wobei man sich bei der Festlegung von Eingriffsart, -stärke und -zeitpunkt an einem idealtypischen Durchforstungsschema sowie den durchschnittlichen Verhältnissen orientiert (vgl. SPELLMANN et al. 1999). Während ein solches Verfahren für die Planung der insgesamt anfallenden Nutzungsmassen rationell und kostensparend ist, liefert es für das waldbauliche Vorgehen in einem konkreten Bestand kaum Entscheidungshilfen, obwohl vor dem Hintergrund anspruchsvoller Waldbaukonzepte Unsicherheiten hinsichtlich der ,richtigen“ Pflege- und Nutzungsentscheidungen bestehen. Zur Verbesserung der Entscheidungsgrundlagen ist die Zielvereinbarung anhand von Weiserflächen (z. B. BÖCKMANN et al. 1998b) allein nicht ausreichend, da diese immer nur Bestandes- 
typen repräsentieren, von denen konkrete Einzelbestände in ihrem Aufbau und Zustand mehr oder weniger abweichen.

Durch die Entkoppelung der Bewirtschaftungseinheit Waldbestand von den Planungsprozessen Vorratschätzung, Nutzungsplanung und Fortschreibung nimmt der Wert der bereits zu Anfang der Planungsperiode ungenauen Bestandesdaten mit der Länge des Fortschreibungszeitraumes immer mehr ab, und zwar um so stärker, je öfter durchforstet wird und je stärker die tatsächlichen von den unterstellten Durchforstungen abweichen. Zudem wird die Prognose der weiteren Bestandesentwicklung verfälscht sein, wenn durch die geschätzten Durchforstungen deutlich abweichende Wuchskonstellationen erzeugt werden (Abb. 1; vgl. a. PretzSCH 1995, 1997; PoMmERENING 1998). Neben der abnehmenden Aktualität der Bestandesdaten läßt sich überdies die Zielkonformität der realisierten Eingriffe erst im Nachhinein beurteilen. Für die zeitnahe Kontrolle der Produktion ist die Betriebsinventur daher kaum geeignet.
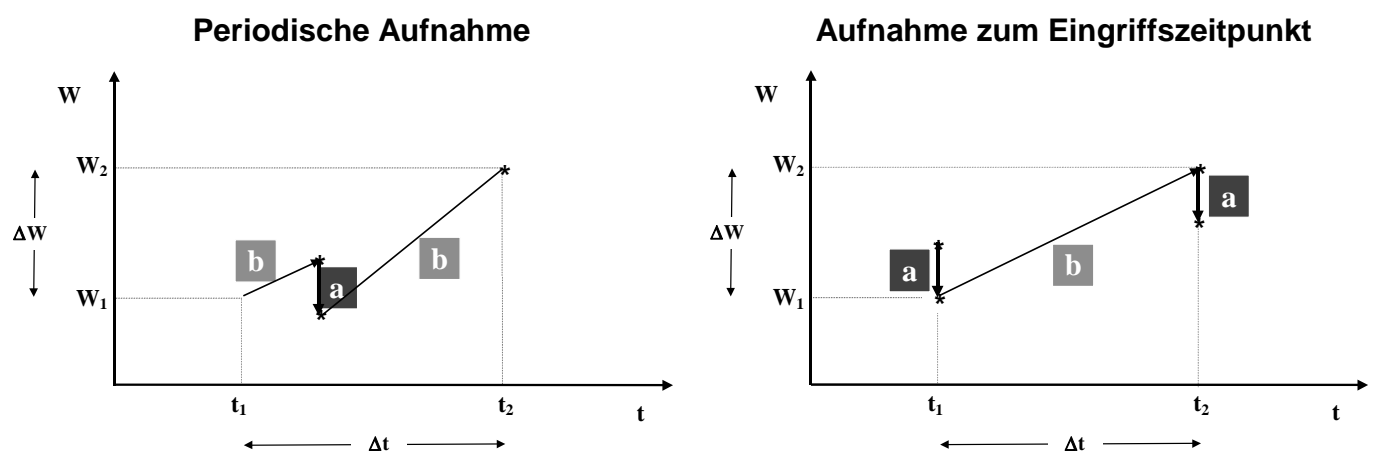

Abb. 1. Zwei aufeinanderfolgende Aufnahmen zur Erfassung der Veränderung der Wachstumsgröße $W$ im Zeitintervall $\Delta t$ (GADOW und SCHMIDT 1998). Links: Bei periodischen Inventuren (Kontrollstichproben) muß die Reaktion auf den Eingriff (a) i.d.R. geschätzt werden. Rechts: Wenn die Inventur im engen zeitlichen Zusammenhang mit dem Eingriff stattfindet, können eingriffsbedingte (a) und zuwachsbedingte (b) Zustandsveränderungen getrennt erfaßt werden.

Two successive inventories for assessing the change of the growth variable $W$ during the time interval $\Delta t$ (GADOW and SCHMIDT 1998). Left: In periodic Inventories, the reaction to the harvest event (a) must be estimated. Right: If the assessment takes places within a short time window around the harvest event, harvest-related (a) and growth-related (b) changes can be identified separately.

Es soll an dieser Stelle auch daran erinnert werden, daß die Kontrollstichprobe primär für die speziellen Verhältnisse der „homogen inhomogenen“ Schweizer Plenterwälder entwickelt wurde, also für Forstbetriebe, die praktisch nur durch einen einzigen Bestand gebildet werden. Hier liefert sie selbstverständlich operationale Informationen und verbessert die Kontrolle der waldbaulichen Maßnahmen. Anders in Betrieben, die sich aus einer Vielzahl von Beständen mit unterschiedlichsten Strukturen zusammensetzen, wie es in Deutschland in der ganz überwiegenden Zahl der Fall ist (GAdow und SchmidT 1998; vgl. a. TzschupKe 1991; SAGL 1995). Daß sich dieser Zustand grundlegend ändern wird, darf bezweifelt werden, da vor dem 
Hintergrund des teilweise drastischen Personalabbaus der letzten 10 Jahre $^{3}$ und der zunehmenden Ausrichtung auch der öffentlichen Forstbetriebe auf ökonomischen Erfolg (HostetTLER 2006) sich in den letzten Jahren wieder Tendenzen zu einem großflächigeren Wirtschaften feststellen lassen (z. B. HOCKENJOS 2006), die der eingangs skizzierten Entwicklung hin zu einer Auflösung der Bestandesgrenzen zuwiderlaufen.

Bestandesbezogene Inventur- und Planungsansätze besitzen den Vorzug, daß sie grundsätzlich die Möglichkeit bieten, die strategische und die operationale Ebene miteinander zu verknüpfen (s. z. B. GADOW und PUUMALAINEN 2000). Als Alternative zu den periodischen Inventurverfahren ist am Institut für Waldinventur und Waldwachstum der Georg-AugustUniversität Göttingen deshalb das Konzept der bestandesbezogenen Eingriffsinventur aufgegriffen und weiterentwickelt worden. Wie der Name bereits sagt, werden solche Inventuren nicht in fest definierten Zeitabständen durchgeführt, sondern immer dann, wenn ein Eingriff bevorsteht (GADOW und STÜBER 1994). Die Aufnahme des Bestandes basiert auf einer temporären, systematischen Stichprobe und erfolgt nach dem Auszeichnen, aber vor der Entnahme der markierten Bäume. Die Vorteile dieses Vorgehens wurden von GADOW und SCHMIDT (1998) ausführlich beschrieben. Vier Punkte sollen hier aber noch einmal besonders hervorgehoben werden 4 :

- Eine Bestandesinventur ermöglicht eine flexible Ausrichtung des Inventuraufwandes am Informationsbedarf und damit eine Optimierung des Kosten-Nutzen-Verhältnisses der Inventuren. So kann durch eine Extensivierung der Datenerhebung in jungen und strukturarmen Beständen die Kostensteigerung durch eine intensivierte Informationsgewinnung in wertvollen Beständen kompensiert werden.

- Durch die gleichzeitige Erfassung des Bestandeszustandes und dessen Veränderung kann die waldbauliche Maßnahme analysiert und gegebenenfalls korrigiert werden (präventive Nachhaltskontrolle).

- Die Zustandsgrößen des verbleibenden Bestandes verändern sich bis zur nächsten Inventur - sofern keine Kalamitäten auftreten - nur durch das natürliche Wachstum und die Mortalität der Bäume, also Prozesse, die inzwischen selbst für ungleichaltrige Mischbestände ziemlich genau vorhergesagt werden können (STERBA 1990; HASENAUER et al. 1995; Nagel 1999, 2000; PretZsch und Kahn 1996; PretZsCh und Dursky 2001; HALLENBARTER et al. 2005).

\footnotetext{
${ }^{3}$ So werden z. B. in den Niedersächsischen Landesforsten im Vergleich zum Stand vor 1997 bis zum Jahr 2008 $40 \%$ der Beamten, Angestellten und Forstwirte eingespart worden sein (MERKER 2006)

4 Eingriffsinventuren werden außerhalb Mitteleuropas mit Erfolg eingesetzt (z. B. KASSIER 1980). Bereits das im Schweizer Kanton Neuchatel verwendete Forsteinrichtungsverfahren der Kontrollmethode weist Elemente einer Eingriffsinventur auf, da neben der periodischen Erfassung des Gesamtbestandes auch der ausscheidende Bestand bei jedem Eingriff mit Hilfe der Stehend-Kluppierung erfaßt wird (GURNAUD 1886).
} 
- In Verbindung mit einer jährlichen, bestandesweisen Aktualisierung der Holzvorratsdaten, basierend auf einer Zuwachsschätzung mit Hilfe von Wuchsmodellen (Fortschreibung), bleibt die Aktualität der Daten über den gesamten Planungszeitraum erhalten.

Aus dem oben gesagten wird deutlich, daß der nach Brusthöhendurchmesserklassen differenzierten Erfassung des ausscheidenden Bestandes eine zentrale Rolle bei der Pflege der Forsteinrichtungsdaten zukommt. Zugleich dient sie der betrieblichen Steuerung und der Optimierung von Holzernte und -absatz (z. B. PuUMALAINEN 1998, S. 3 f.). Mögliche Anwendungsbereiche sind z. B. (vgl. RHEINLAND-PFÄLZISCHES FORSTAMT DAUN 2000):

- Optimierung der Sortenaushaltung und der Arbeitsverfahren

- Optimierung der Zuordnung von Lieferaufträgen zu Hieben und Optimierung der Blockbildung bei der Maßnahmenplanung

- Aufstellung von zuverlässigen Hiebs-, Verkaufs- und Finanzplänen

- Möglichkeit des Stockverkaufs mit differenzierter Preisbildung und Rechnungsausstellung vor Aufarbeitungsbeginn

- Verbesserte Ausschreibung und Vergabe von Lohnunternehmeraufträgen

Die Erfassung des ausscheidenden Bestandes, die Weiterverarbeitung dieser Daten und ihre Integration in den vorhandenen Datenbestand ist damit ein wichtiger Bestandteil der (forstbetrieblichen) Logistik, die nach SCHÖNSLEBEN (2000) die „Organisation, Planung, Realisierung und Steuerung des gesamten Informations- und Materialflusses entlang der ganzen Wertschöpfungskette“ bezeichnet. Innerhalb dieses Systems werden die drei Komponenten physische, administrative und dispositive Logistik unterschieden. Die Inventur der Holznutzungen ist sowohl der administrativen, als auch der dispositiven Logistik zuzuordnen, da sie einerseits dem „Erfassen, Aufbereiten, Verarbeiten, Transportieren und Speichern der für einen geordneten Produktionsablauf erforderlichen Informationen“ dient, zugleich aber auch die Datengrundlage für die „Entscheidungssituationen“ liefert, „die für eine effektive und effiziente Steuerung des Gesamtablaufes benötigt werden“ (SCHÖNSLEBEN 2000).

HeINIMANN (1999) weist darauf hin, daß die Wandlung des Holzmarktes von einem Verkäufer- zu einem Käufermarkt Produktionsprozesse erfordert, welche sich viel stärker als bisher an den Bedürfnissen des Marktes orientieren. Herzstück einer kundenorientierten Rohholzbereitstellung sollte daher die Auftragsliste sein. Zur Optimierung eines solchen Produktionsprozesses sind jedoch genauere Informationen über die Sortenzusammensetzung innerhalb der Bestände erforderlich, da das Kernproblem der dispositiven Logistik darin besteht, die aufzuarbeitende Auftragsliste mit der in den Holzschlägen vorhandenen Rohstoffbasis abzugleichen (BERGMANN 1997). Interessante Ansätze zur Lösung dieses Problems wurden 
z. B. von Uusitalo (1995), Uusitalo und Kivinen (2000) und Nieuwenhuis et al. (2001) vorgestellt. Ziel dieser Arbeit ist es, ein Verfahren der permanenten Inventur zu entwickeln, das in die betrieblichen Abläufe und Informationsflüsse vollständig integriert ist, eine flexible Anpassung an den tatsächlichen Informationsbedarf erlaubt und über die Mehrfachnutzung der erhoben Daten sowohl der Zustandserfassung im Rahmen der Forsteinrichtung, als auch dem forstbetrieblichen Controlling und der Optimierung von Produktion und Absatz dient.

\section{Das Prinzip der Eingriffsinventur}

In dem von GADOW und STÜBER (1994) und GADOW und SCHMIDT (1998) vorgestellten Verfahren ist die gleichzeitige Erfassung des ausscheidenden und verbleibenden Bestandes durch eine Stichprobeninventur nach dem Auszeichnen und vor der Ausführung des Eingriffs vorgesehen. Die Vorteile, die sich aus diesem Vorgehen ergeben, wurden bereits erläutert. Ein besonders wichtiger Aspekt ist in diesem Zusammenhang die Beurteilung der eingriffsbedingten Veränderung von Vorrat, Struktur und Wert. Durch die gleichzeitige Erfassung von verbleibendem und ausscheidendem Bestand verhält sich eine solche Inventur bezüglich der Zustandsveränderung wie eine permanente Stichprobe, deren Vorteil darin besteht, daß sie die Änderungsrate mit einem geringeren Fehler schätzt als temporäre Stichproben (AKÇA 2001, S. 131). Gleichzeitig ergeben sich aber auch nicht unwesentliche Nachteile:

- Da in der Vornutzung nur sehr selten mehr als $15 \%$ des Vorrats entnommen werden, wird der ausscheidende Bestand i.d.R. mit einem nur kleinen Anteil in die Stichprobe eingehen. Dadurch ist der Stichprobenfehler für dieses Kollektiv relativ hoch, was den Nutzen dieser Art der Datenerhebung in Frage stellt.

- Der betriebliche Organisations- und Abstimmungsaufwand ist durch das enge Zeitfenster, innerhalb dessen die Inventur der Bestände erfolgen muß, recht hoch. Unter der Annahme, daß der Umfang der „Just-in-time“-Holzernte in Zukunft weiter zunehmen wird, ist es wahrscheinlich, daß die Durchführung der Inventur in vielen Fällen nicht rechtzeitig möglich sein wird.

Aus diesen Gründen wird das in Abb. 2 illustrierte Vorgehen mit zeitlich getrennter Erfassung von verbleibendem und ausscheidendem Bestand vorgeschlagen. Demnach sollte während des Auszeichnens jeder zu entnehmende Baum auch gekluppt werden. Diese vollständige Erfassung des ausscheidenden Bestandes erfordert nur geringen Mehraufwand, wenn hierfür eine elektronische Kluppe eingesetzt wird, mit der jeder Durchmesser getrennt nach Baumart gespeichert und später über eine geeignete Schnittstelle auf den PC übertragen werden kann. Die baumartenspezifischen Durchmesserverteilungen des ausscheidenden Bestandes werden auf diese Weise - solange keine Meßfehler auftreten - absolut zuverlässig und ohne jeden 
Stichprobenfehler erfaßt. Je nach Informationsbedarf und Genauigkeitsansprüchen kann diese Grunddatenerfassung erweitert werden.

Ein in der Praxis bereits bewährtes Arbeitsverfahren wurde durch das RHEINLANDPfÄLZISCHE FORSTAMT DAUN (2000) vorgestellt. Die bei diesem Verfahren angestrebte genaue Schätzung der anfallenden Sortimente mit Hilfe des Programms Holzernte (SCHÖPFER 1998) macht die zusätzliche Messung von ca. 20 - 40 Baumhöhen zur Erstellung einer bestandesindividuellen Höhenkurve erforderlich. Allerdings sollte geprüft werden, ob die Verwendung einer Einheitshöhenkurve nicht eine ausreichende Genauigkeit liefert. Hierdurch ließe sich die Anzahl der arbeitsintensiven Höhenmessungen deutlich reduzieren, da zur Ermittlung der dann benötigten Bestandesmittelhöhe nur 5-10 Baumhöhen gemessen werden müßten.

Ist die Vollkluppung des ausscheidenden Bestandes (z. B. in sehr jungen und stammzahlreichen Beständen) nicht möglich oder sinnvoll, kann deren Durchmesserverteilung geschätzt werden. Hierfür stehen zwei alternative Methoden zur Verfügung, die allerdings keine vorherige Kontrolle des Eingriffs erlauben: Wurde der Bestand mit dem Harvester aufgearbeitet, kann die Durchmesserverteilung der entnommenen Bäume aus den im Bordcomputer gespeicherten Daten ohne großen Aufwand generiert werden. Bei motormanueller Durchforstung kann die Schätzung mit Hilfe des auf der Weibullfunktion basierenden Ansatzes von Staupendahl (1999) erfolgen (vgl. a. STAUPENDAhl und PuUMALAinen 1999). Als Eingangsgrößen werden hierbei lediglich der kleinste Durchmesser, der Durchmesser des Grundflächenmittelstamms und die Stammzahl des Gesamtbestandes, sowie der Quotient aus den Entnahmequoten der Stammzahl und Grundfläche benötigt, wobei der letztgenannte Parameter die Durchforstungsart beschreibt und somit die Abbildung sehr unterschiedlicher Eingriffe erlaubt.

Falls für den verbleibenden Bestand keine Daten vorliegen oder die vorhandenen Daten veraltet sind (s. a. Abschnitt 3.1), erfolgt nach dem Einschlag dessen Neuinventur. Wenn diese auf einem Stichprobenverfahren basiert, wird im Vergleich mit der Methode von GADOW und STÜBER (1994) für jeden nicht aufgenommenen ausscheidenden Baum ein zusätzlicher Baum des verbleibenden Bestandes in die Stichprobe eingehen, so daß dieser genauer erfaßt wird bzw. die geforderte Genauigkeit mit einem geringeren Inventuraufwand eingehalten werden kann. Dies ist von einiger Bedeutung für die Fortschreibung der Bestandesdaten, da die Qualität der Prognose selbstverständlich nicht nur von der Validität der verwendeten Wuchsmodelle, sondern mindestens ebenso von der Genauigkeit der Zustandserfassung abhängt. Aus Gründen der besseren Begehbarkeit des Bestandes ist die Durchführung der Inventuren vor dem Einschlag natürlich wünschenswert. Allerdings müssen dann die zur Nutzung vorgesehen Bäume bereits markiert sein, damit die alleinige Erfassung des verbleibenden Bestandes möglich ist. Und selbst dann besteht natürlich die Gefahr, daß später nicht genau die markierten Bäume entnommen werden. 


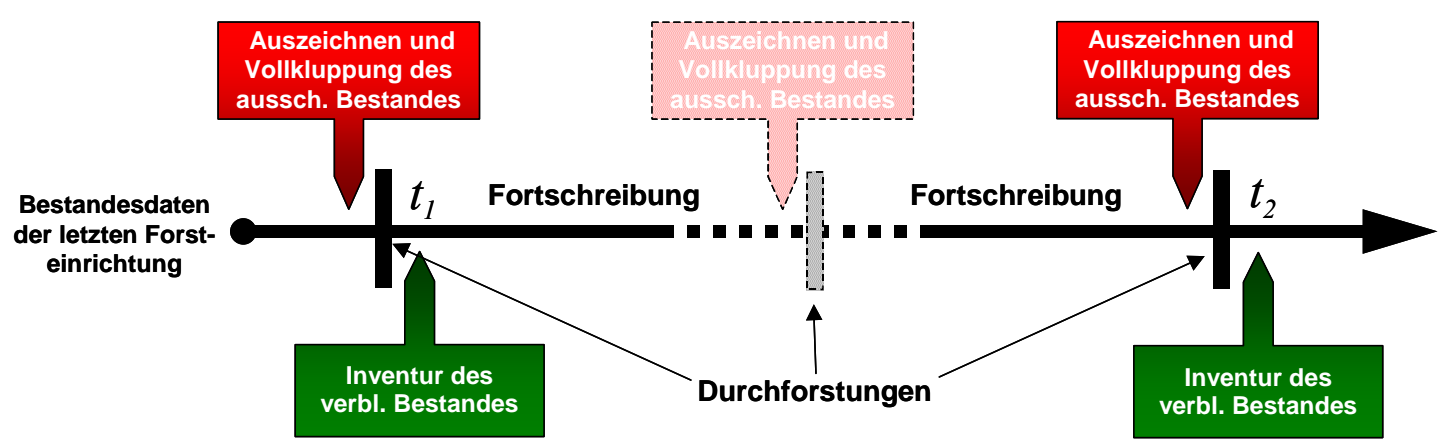

Abb. 2. Ablaufschema der Eingriffsinventuren entlang der Zeitachse. Die vertikalen Balken markieren den Zeitpunkt der Hiebsmaßnahmen. Das Intervall zwischen den Inventuren des verbleibenden Bestandes ist bestandesindividuell und sollte in Abhängigkeit von der Wuchsdynamik und Durchforstungshäufigkeit zwischen 15 und 25 Jahren liegen (s. a. Abschnitt 3.2). Die Fortschreibung zwischen den Inventuren erfolgt mit Hilfe eines Wuchsmodells (vgl. NAGEL 1998).

Succession of possible harvest event assessments along the timeline. The vertical bars show the point in time of a harvest event. The interval between the assessments of the remaining stand should be between 15 and 25 years, depending on the stand condition and the frequency of thinnings (see section 3.2). The development of the growing stock between the assessments is estimated using a growth model (cf. NAGEL 1998).

Die Daten des verbleibenden Bestandes werden nun jedes Jahr bis zur nächsten Inventur mit Hilfe eines Wachstumssimulators fortgeschrieben. Zwischenzeitlich stattfindende Eingriffe werden wie beschrieben durch Vollkluppung während des Auszeichnens (oder alternative Verfahren) erfaßt und in die Datenbank importiert. Die Durchmesserverteilungen des ausscheidenden Bestandes werden nach Darstellung am Bildschirm von den fortgeschriebenen Durchmesserverteilungen subtrahiert und der Datenbestand dadurch aktualisiert. Die hieraus resultierenden Daten werden anschließend weiter fortgeschrieben. Auf diese Weise werden mehrere Zwecke erfüllt:

- Jeder Eingriff kann ziemlich genau analysiert werden. Der auszeichnende Förster erhält dadurch objektive Informationen über die Auswirkungen seines waldbaulichen Vorgehens und kann es (falls der ausscheidende Bestand vor dem Eingriff erfaßt wurde) gegebenenfalls korrigieren.

- Es stehen jederzeit aktuelle Zustandsdaten der Bestände und damit des Forstbetriebes zur Verfügung.

- Die Erfassung des ausscheidenden Bestandes dient nicht nur der Optimierung von Holzernte und Absatz, sondern zugleich der Aktualisierung und Fortschreibung der Bestandesdaten. Umgekehrt können Daten aus der Inventur des verbleibenden Bestandes für die Kalkulation der anfallenden Sortimente genutzt werden: Unter der Vorraussetzung, daß sich das durchschnittliche H/D-Verhältnis der Bäume im verbleibenden und ausscheidenden Bestand nicht wesentlich unterscheidet, könnte z. B. die zusätzliche Messung von Baumhöhen entfallen, da diese über die Höhenkurve des verbleibenden Bestandes geschätzt werden könnten (s. Abschnitt 3.4). 
- Durch die langfristige Aufzeichnung der Bestandesentwicklung entsteht ein wertvoller Datenfundus, der für vielfältige Zwecke (z. B. die lokale Kalibrierung der verwendeten Wuchsmodelle) genutzt werden kann.

Durch diese Mehrfachnutzung der Daten wird die Wirtschaftlichkeit der Inventuren erheblich gesteigert.

\section{Die Inventur des verbleibenden Bestandes}

\subsection{Auswahl des Inventurverfahrens}

Will man das Kosten-Nutzen-Verhältnis der Waldinventur optimieren (Abb. 3), sollte nicht nur das Inventurintervall, sondern auch die Art der Bestandeserfassung an den tatsächlichen Informationsbedarf angepaßt werden. So erscheint z. B. der Aufwand einer Stichprobenerhebung in jungen, gleichaltrigen Reinbeständen - gemessen am Wert der zusätzlich gewonnenen Informationen - nicht gerechtfertigt. Unter betriebswirtschaftlichen Gesichtspunkten steigt der Informationsbedarf normalerweise mit steigendem Stammholzanteil. Neben Beständen, die aus anderen Gründen von besonderer Bedeutung sind (Sonderbiotope, Flächen des Vertragsnaturschutzes, etc.), sollten deshalb je nach Baumart nur Bestände mit einem mittleren Durchmesser von mind. $25-30 \mathrm{~cm}$ mit einer Stichprobeninventur erfaßt werden (s. Abschnitt 3.4).

In allen anderen Fällen ist der Waldbegang mit einer schichten- und baumartenspezifischen Schätzung von Grundfläche pro ha, Mittelhöhe und Mitteldurchmesser, oder sogar nur eine summarische Erfassung und Planung auf der Basis von Bestandestypen ${ }^{5}$ eine angemessene Alternative (vgl. z. B. SPELLMANN und AKÇA 1983). Die auch in diesen Wirtschaftseinheiten interessierende Durchmesserverteilung kann mit der von GAFFREY et al. (1998) vorgestellten Methode, die die Normal- bzw. Weibullverteilung nutzt und deren Parameter anhand von Bestandesmittelwerten vorhersagt, relativ genau geschätzt werden (vgl. a. NAGEL und BIGING 1995).

\footnotetext{
${ }^{5}$ Als Bestandestypen werden hier nach Bestandesstruktur, Baumartenzusammensetzung, Bestandesqualität und natürlicher Altersstufe zusammengefaßte Straten bezeichnet.
} 


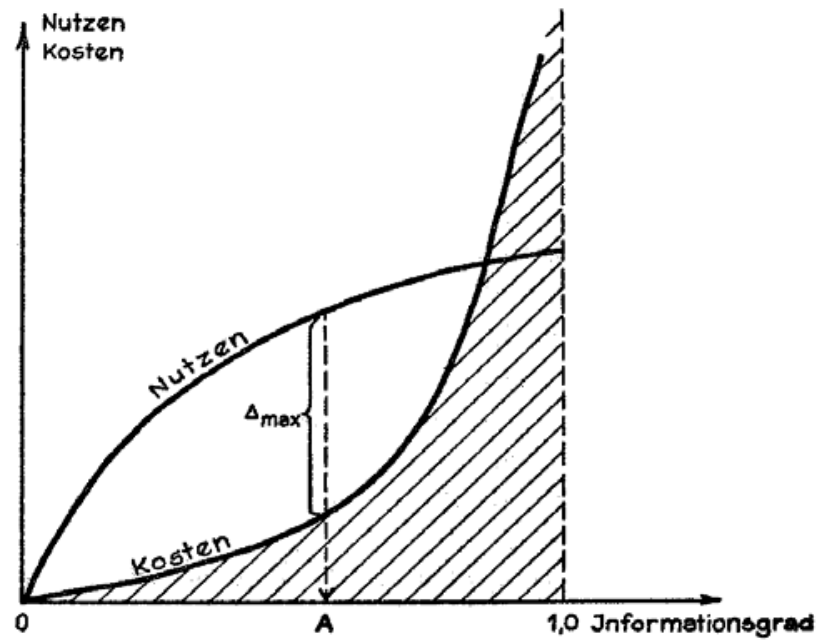

Abb. 3. Bestimmung des optimalen Informationsgrades $(A)$ mit Hilfe der Nutzen- und Kostenfunktion (SPEIDEL 1972, S. 31).

Determining the ideal "information level" $(A)$, based on the benefit-cost relationship (SPEIDEL 1972, p. 31).

Die Entscheidung, welches Verfahren gewählt wird, liegt jedoch vollkommen im Ermessen des Forstbetriebes und sollte sich allein an dessen Zielen sowie am Verhältnis von Kosten und Nutzen der Informationen orientieren. Abb. 3 stellt hierfür ein Kalkül zur Verfügung. Demnach ist derjenige Informationsgrad optimal, der den größten Nutzen(/Ertrags)-Überschuß aus den vorhandenen Informationen über deren Beschaffungskosten gewährleistet. Die besondere Schwierigkeit besteht allerdings darin, den Nutzen zu quantifizieren. Hierfür müßten die Auswirkungen zusätzlicher Informationen auf das Betriebsergebnis abgeschätzt werden, was angesichts der Komplexität der Zusammenhänge und v. a. der Länge der Produktionszeiträume nur sehr ungenau gelingen kann. Zur Klärung dieser Fragen wären daher weitere Untersuchungen dringend erforderlich.

Abb. 4 zeigt einen Vorschlag für einen Kriterienkatalog, der bei der Entscheidung über Zeitpunkt und Art der Inventur als Orientierungshilfe dienen könnte. U. a. sollte berücksichtigt werden, daß eine Stichprobeninventur aufgrund der relativ hohen Kosten i.d.R. nur in Beständen ab einer Größe von 5-10 ha wirtschaftlich vertretbar ist (vgl. NICKE 2005). Erfüllt ein einzelner Bestand diese Voraussetzung nicht, sollte geprüft werden, ob es innerhalb des Forstbetriebes ähnliche strukturierte Bestände gibt, die zusammengefaßt eine ausreichende Größe erreichen. Unabhängig vom gewählten Inventurverfahren gilt jedoch immer das „Eingriffsprinzip“, d. h. das in Abschnitt 2 vorgestellte Verfahren der getrennten (und vollständigen) Erfassung des ausscheidenden Bestandes, die zeitliche Orientierung der Inventur am Eingriff und die Fortschreibung der Bestandesdaten. 


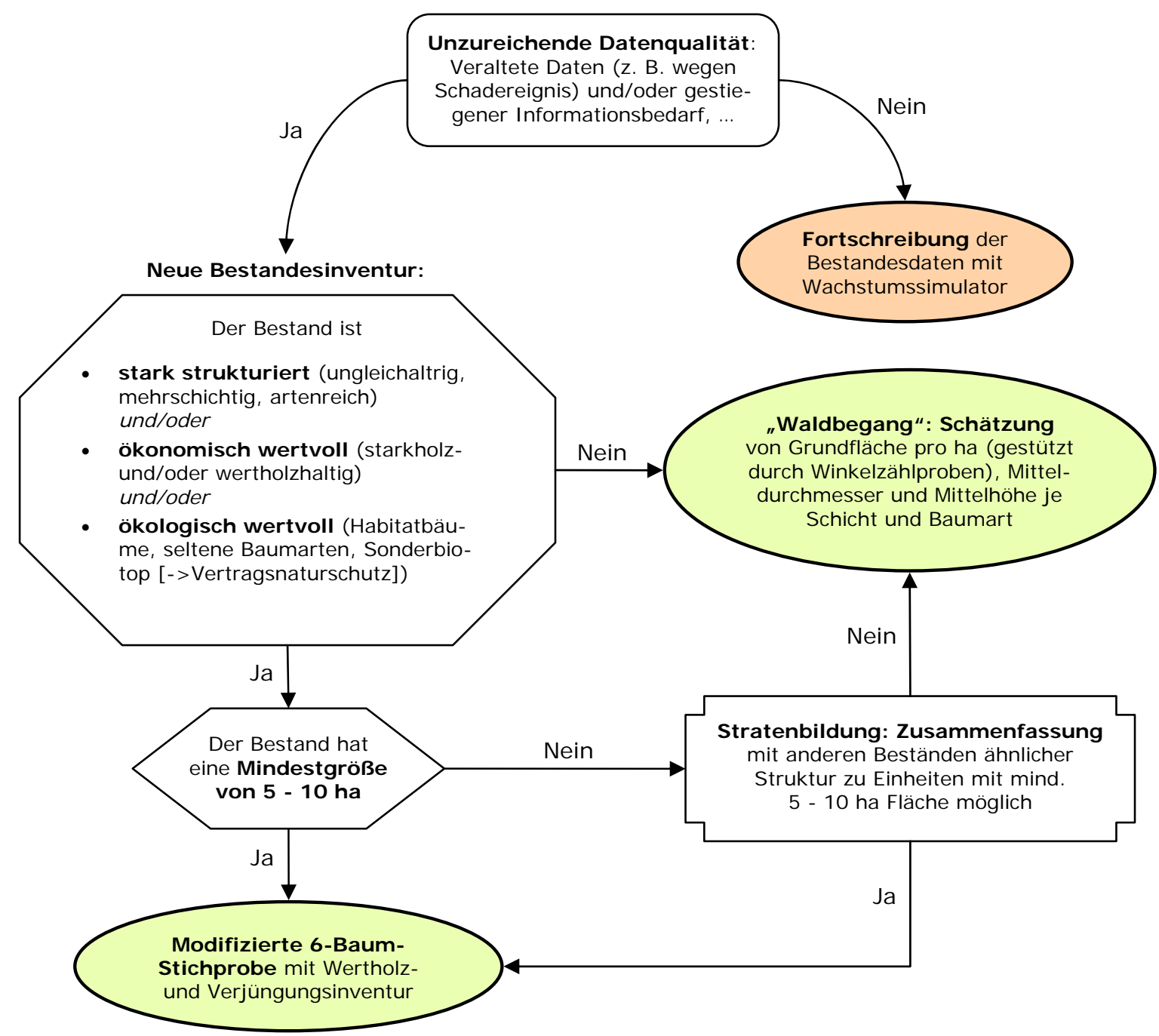

Abb. 4. Entscheidungsbaum als Hilfestellung zur Beantwortung der Frage, ob und wie die Daten eines Bestandes (verbleibender Bestand) neu erhoben werden sollen.

Decision tree for evaluating the need for stand assessment and the method that should be employed.

\subsection{Inventurintervall}

Entsprechend dem in Abb. 2 dargestellten Schema könnte die Inventur des verbleibenden Bestandes bereits nach jedem nächsten Eingriff wiederholt werden. Dies würde jedoch bei den üblichen Durchforstungsintervallen zu nicht vertretbaren Inventurkosten führen. Darüber hinaus sollte bei der Wahl des Inventurintervalls auch darauf geachtet werden, daß die Veränderung der hauptsächlich interessierenden Variablen mit einem angemessenen relativen Fehler geschätzt werden. Als wichtigsten Variablen werden hier die Mittelhöhe, der Mitteldurchmesser und die Bestandesgrundfläche betrachtet. Letztere steht in einem linearen $\mathrm{Zu}$ sammenhang zum Bestandeswert und ist - im Gegensatz zur berechneten Variable Holzvorrat - direkt meßbar. Nach den Regeln der Fehlerfortpflanzung ist bei temporären Stichproben der absolute Fehler der Veränderung eines Merkmals zwischen zwei Inventuren gleich der Quadratwurzel aus der Summe der Varianzen der Zustandsschätzungen (AKÇA 2001, S. 30 f.). 
Eine Folgeinventur sollte also nicht stattfinden, wenn sich die interessierenden Variablen aufgrund geringen Zuwachses und/oder kompensatorisch wirkender Nutzungen kaum verändert haben, da in einem solchen Fall bei vertretbarem Stichprobenumfang die Schätzung der Veränderung im Stichprobenfehler ,untergeht“ und damit vollkommen unklar ist, ob die festgestellte Zustandsänderung der Wirklichkeit entspricht oder durch Zufallseffekte der Stichprobenziehung nur suggeriert wird. Wenn der angestrebte relative Stichprobenfehler (Standardfehler) der Zustandsgrößen aus wirtschaftlichen Gründen auf einen bestimmten Wert festgelegt ist, wird der relative Fehler der Schätzung der Zustandsänderung also erst dann ein vertretbares Maß erreichen, wenn die Änderungsrate ausreichend groß ist. Von Schadereignissen abgesehen wird dies je nach Wuchsdynamik eines Bestandes nach unterschiedlichen Zeiträumen der Fall sein.

Wenn man die wahren Werte der zu schätzenden Variablen als Bezugsgrößen verwendet, lautet die Formel zur Berechnung des relativen Standardfehlers der Zustandsänderung:

$$
\begin{aligned}
& s_{\overline{\Delta X}} \%=\frac{\sqrt{s_{\bar{X}_{1}}{ }^{2}+s_{\bar{X}_{2}}{ }^{2}}}{\bar{X}_{2}-\bar{X}_{1}}=\frac{s_{\bar{X}} \% \cdot \sqrt{\bar{X}_{1}^{2}+\bar{X}_{2}^{2}}}{\bar{X}_{2}-\bar{X}_{1}} \\
& \text { mit } \bar{X}_{1}, \bar{X}_{2}=\text { Schätzwerte der Variablen } X \text { zum Zeitpunkt } 1 \text { (Erstinventur) und } 2 \text { (Folgeinventur) } \\
& s_{\bar{X}_{1}}, s_{\bar{X}_{2}}=\text { absolute Standardfehler der Variablen } X \text { zu den Zeitpunkten } 1 \text { und } 2 \\
& \begin{array}{ll}
s_{\bar{X}} \% & =\text { konstanter relativer Standardfehler der Variablen } X=s_{\bar{X}_{1}} / \bar{X}_{1}=s_{\bar{X}_{2}} / \bar{X}_{2} \\
\overline{\Delta X} & =\bar{X}_{2}-\bar{X}_{1}
\end{array}
\end{aligned}
$$

Aus (1) folgt, daß die nächste Inventur um so später erfolgen sollte, je größer die Werte der Zielvariablen $\left(\bar{X}_{1}^{2}+\bar{X}_{2}^{2}\right)$ und deren relative Standardfehler $\left(s_{\bar{X}} \%\right)$ sind und je weniger sich die Variablenwerte ändern $\left(\bar{X}_{2}-\bar{X}_{1}\right)$. Abb. 5 verdeutlicht diese Zusammenhänge anhand von exemplarischen Daten aus der SCHOBER'schen Ertragstafel für Buche, I. Ertragsklasse, mäßige Durchforstung (SCHOBER 1995). Sie zeigt den mit Formel (1) berechneten Standardfehler der Zuwachsraten der Zielgrößen für Folgeinventuren in unterschiedlichen Zeitabständen zu zwei Erstinventuren im Bestandesalter 40 und 65. Bei der Kalkulation wurde ein Standardfehler der Zustandsschätzung von $10 \%$ unterstellt. Stichprobensimulationen mit dem in Abschnitt 3.4 beschriebenen Verfahren haben ergeben, daß dieser Fehlerwert auch in strukturreichen Beständen mit ca. 20-40 Probekreisen je Bestand eingehalten werden kann (STAUPENDAHL 2008), so daß es sich hierbei um eine realistische Vorgabe handelt.

Wird das Doppelte des Fehlers der Zustandsschätzung, also $20 \%$, als ein tolerabler Fehler der Zuwachsschätzung betrachtet, so wird dieser Wert bei der Grundflächenzuwachsschätzung innerhalb des Produktionszeitraumes weder für die Erstaufnahme im Alter 40 noch für die Aufnahme im Alter 65 jemals erreicht. Bei der Höhenzuwachsschätzung wird er ab einem Inventurintervall von 50 Jahren unterschritten, wenn der Bestand im Alter 40 zum ersten Mal aufgenommen wurde, bei einer Erstinventur im Alter 60 liegen die Fehlerwerte 
ebenfalls immer über $20 \%$. Lediglich bei der Schätzung des mittleren Durchmesserzuwachses nähern sich die Inventurintervalle mit knapp 30 Jahren (Erstinventur im Alter 40) bzw. ca. 55 Jahren (Erstinventur im Alter 65) allmählich dem in der Forsteinrichtung üblichen Inventurabstand von 10 Jahren an. Die Sicherheit, daß der wahre Zuwachs dann tatsächlich um nicht mehr als $20 \%$ vom Schätzwert abweicht, beträgt allerdings nur ca. $68 \%$, da kein erweitertes Vertrauensintervall berechnet wurde.

Diese Intervalle ließen sich drastisch verkürzen, wenn der geforderte Fehler der Zustandsschätzung reduziert würde. Allerdings würde damit der notwendige Stichprobenumfang überproportional ansteigen, was aus wirtschaftlichen Gründen nicht akzeptabel ist. Diese Zusammenhänge, die in ähnlicher Größenordnung auch für die anderen Hauptbaumarten gelten, zeigen, daß bei temporären Stichproben auf Bestandesebene und unter den in Deutschland üblichen Durchforstungsintensitäten und Wuchsbedingungen eine Wiederholungsinventur nach weniger als 20 Jahren eigentlich nutzlos ist. Mit Rücksicht auf die Fortschreibung sollte das Inventurintervall aber auch nicht wesentlich länger sein, da sich die Zuverlässigkeit der Wachstumsprognose mit länger werdendem Fortschreibungszeitraum deutlich verschlechtert.

Zusammenfassend kann also ein Inventurintervall zwischen 15 und 25 Jahren empfohlen werden. Diese langen Inventurintervalle und das damit verbundene Einsparpotential im Bereich der Datenerhebung werden jedoch erst durch die genaue Erfassung der Nutzungen und deren Einarbeitung in die mit Wuchsmodellen fortgeschriebenen Bestandesdaten möglich. Die Lösung von starren Inventurintervallen erlaubt es, den Inventuraufwand an die individuellen Gegebenheiten anzupassen, wodurch überflüssige Inventurarbeiten vermieden werden, die keinen oder nur einen sehr geringen Informationsgewinn bringen. Auf der anderen Seite kann genau dort eine höhere Genauigkeit erzielt werden, wo sie notwendig und erwünscht ist.

Natürlich stellt sich in diesem Zusammenhang auch die Frage nach permanenten Stichproben, deren Zuwachsschätzung bei gleichem Stichprobenumfang i.d.R. erheblich genauer ist, wenngleich die Kosten je Probefläche um einiges höher sind, da jede von ihnen genau eingemessen und dauerhaft markiert werden muß. Diese zusätzlichen Kosten können jedoch durch einen geringeren notwendigen Stichprobenumfang mehr als kompensiert werden. $\mathrm{Ob}$ dies der Fall ist, hängt entscheidend davon ab, wie stark die Probekreiswerte der Erst- und Folgeinventur korreliert sind (vgl. AKÇA 2001, S. 132). Bei Wachstumsgrößen wird diese Korrelation um so deutlicher sein, je kleiner das Inventurintervall ist und je weniger die Bestandesstruktur innerhalb dieses Zeitraumes durch Nutzungen oder Störungen verändert wurde. Aus Kostengründen verbietet sich allerdings ein kurzes Inventurintervall und in intensiv bewirtschafteten Wäldern, also gerade dort, wo Eingriffsinventuren interessant sind, werden die nutzungsbedingten Veränderungen nicht unerheblich sein. Aus diesen Gründen wird im Rahmen von Eingriffsinventuren, von wenigen Ausnahmen abgesehen, vermutlich die temporäre Stichprobe effizienter sein. 


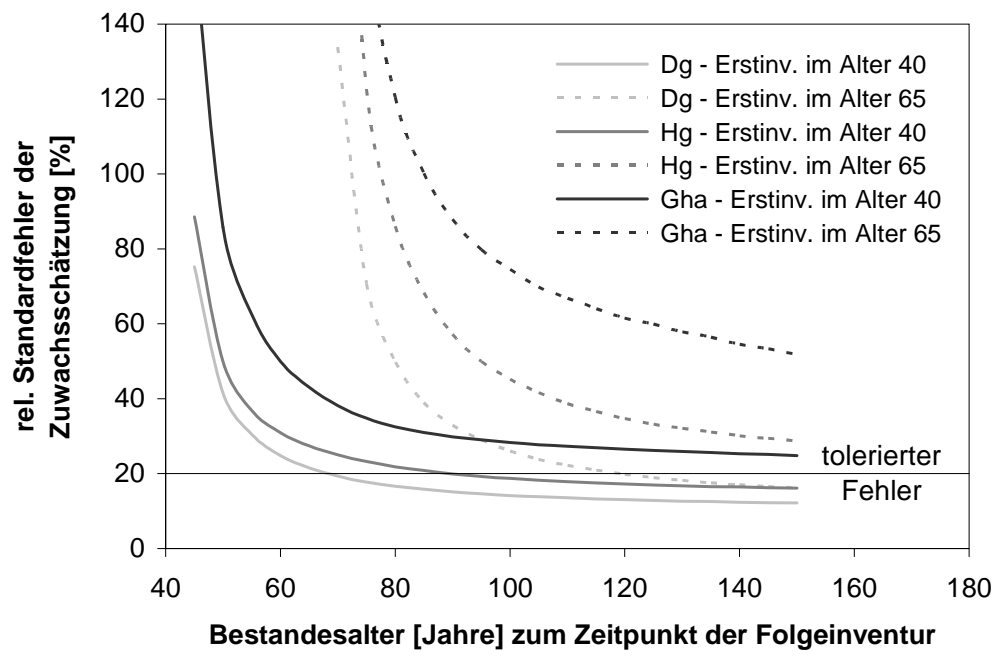

Abb. 5. Theoretischer Standardfehler der Schätzung des Zuwachses von Mitteldurchmesser (Dg), Mittelhöhe $(H g)$ und Grundfläche pro ha $(G h a)$ in Abhängigkeit vom Inventurintervall, dargestellt für zwei Erstinventuren im Bestandesalter 40 und 65, bei einem angenommenen Standardfehler der Zustandsschätzungen von $10 \%$. Die Fehlerberechnung beruht auf den Tafelwerten für den verbleibenden Bestand der SCHOBER'schen Ertragstafel für Buche (I. Ertragsklasse, mäßige Durchforstung).

Theoretical standard error of the increment of the mean diameter $(\mathrm{Dg})$, mean height $(\mathrm{Hg})$ and basal area per hectare $(G h a)$, depending on the interval between successive assessments, shown separately for two initial inventories at the stand ages 40 and 65 years, respectively. It was assumed that the standard error of estimation equals $10 \%$. The calculations are based on the values for the remaining stand using the yield table for beech by SCHOBER (1995; I. yield class, moderate thinning).

\subsection{Inventurvorbereitung}

Praktische Tests ergaben, daß auch bei den hier vorgeschlagenen temporären Stichproben eine Arbeitskarte mit den eingezeichneten Stichprobenpunkten die Orientierung im Gelände erheblich erleichtert und damit den gesamten Ablauf der Außenaufnahmen beschleunigt. Liegen kartographische Daten in digitaler Form vor, so sind diese Unterlagen mit Hilfe der in Abschnitt 4 beschriebenen Software einfach und schnell erstellt. Abb. 6 zeigt die Benutzeroberfläche des entsprechenden Programmoduls nach Generierung der Stichprobenpunkte für die Abt. 12a des Rheinland-Pfälzischen Forstamtes Daun.

Die Verteilung der Stichprobenpunkte auf der Inventurfläche erfolgt bei diesem Verfahren mit Hilfe eines Algorithmus, der ein quadratisches Gitternetz erzeugt. Dieses Gitternetz wird durch drei Parameter definiert, von denen die ersten beiden frei eingegeben werden können: 1.) Die Gitterweite, die bei gegebener Bestandesfläche den Stichprobenumfang bestimmt, 2.) den Ausrichtungswinkel und 3.) den Startpunkt des Gitters, der entweder zufällig erzeugt oder vom Anwender per Mausklick auf der Karte ausgewählt wird. Wurde kein Ausrichtungswinkel angegeben, wird dieser durch einen zweiten Mausklick festgelegt. Die Lage der Stichprobenpunkte wird anschließend angezeigt und kann als Parameterdatensatz gespeichert werden. Durch Abrufen dieser Parameter kann ein einmal gewähltes Gitternetz jederzeit rekonstruiert und z. B. bei späteren Inventuren wiederverwendet werden. Sollte in Einzelfäl- 
len die Durchführung permanenter Stichproben gewünscht sein, ist deren Vorbereitung auf diese Weise sehr erleichtert. Mit Hilfe einer Karte, wie sie in Abb. 6 dargestellt ist, kann sich das Inventurpersonal hervorragend orientieren und die Laufwege optimieren. Neben der Beschleunigung der Inventurarbeiten wird so auch gewährleistet, daß der Bestand tatsächlich repräsentativ erfaßt wird.

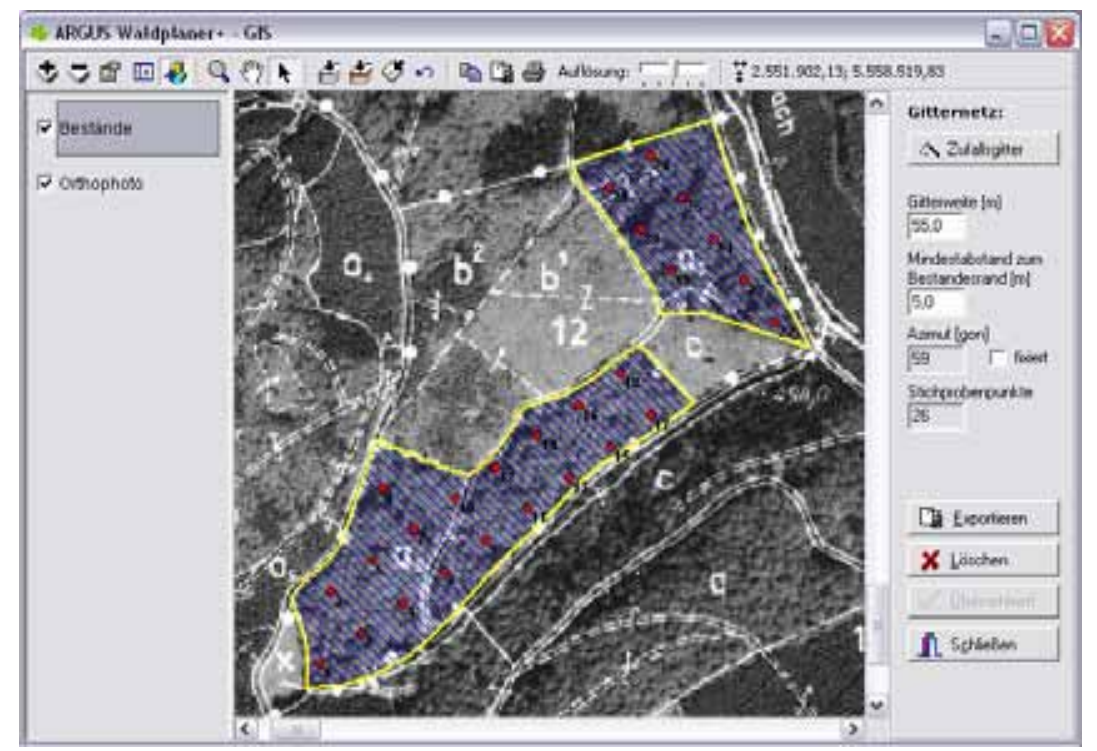

Abb. 6. Verteilung der Stichprobenpunkte mit Hilfe des Programms ARGUS Waldplaner+, dargestellt am Beispiel der Abt. 12a des Forstamtes Daun. Das Gitternetz kann gespeichert und später bei der Vorbereitung weiterer Inventuren wiederverwendet werden. Ebenso können die Koordinaten der Stichprobenpunkte in eine Textdatei exportiert und die Karte in einer Grafikdatei gespeichert oder auf einem Drucker ausgegeben werden.

Allocation of sample points using the software ARGUS Waldplaner+. The example shows the compartment 12a in the forest district Daun. The sample grid can be saved und reused for the preparation of further assessments. The coordinates of the sample points can be exported to a text file and the map can be sent to various other devices.

\subsection{Die modifizierte 6-Baum-Stichprobe}

Das bisher für Eingriffsinventuren favorisierte Stichprobenverfahren basiert auf einer Kombination aus modifiziertem Stammabstandsverfahren und Winkelzählprobe. Hierbei werden u. a. zur Schätzung des Mitteldurchmessers an jedem Stichprobenpunkt der nächstgelegene Baum (Nullbaum) und seine drei nächsten Nachbarn erfaßt, während die Grundfläche pro ha mit einer bestimmten Anzahl Winkelzählproben geschätzt wird (POMMERENING und SCHMIDT 1998). In den meisten der bisher untersuchten Bestände liefert dieses Verfahren gute Schätzungen. Eine genaue Analyse mit Hilfe von Stichprobensimulationen zeigte jedoch, daß immer dann mit einem z. T. erheblichen systematischen Fehler zu rechnen ist, wenn innerhalb eines Bestandes räumlich voneinander getrennte Subpopulationen mit unterschiedlicher Dichte und Durchmesserverteilung auftreten. 
Da jedoch gerade diese Strukturen in den angestrebten ungleichaltrigen, femelartig aufgebauten Mischwäldern zu erwarten sind, wurde als alternatives Verfahren die auf der Messung von Punkt-Baum-Abständen beruhende 6-Baum-Stichprobe (PRODAN 1968) untersucht. Die 6-Baum-Stichprobe ist als einfach anzuwendendes und kostengünstiges Inventurverfahren bekannt, das - im Gegensatz zu Inventuren mit festen bzw. konzentrischen Probekreisen keinen Spezialisten, sondern nur den eingeübten Mitarbeiter der Forsteinrichtung erfordert (HIRNER 1978, S. 2). Insbesondere kann es als Ein-Personen-Verfahren angewandt werden, was die Inventurkosten erheblich reduziert (vgl. NiCKE 2005). Für die Anwendung im Rahmen von Eingriffsinventuren ist es daher besonders geeignet.

Bei der 6-Baum-Stichprobe ist die Stichprobeneinheit definiert durch die vom Stichprobenpunkt $i$ aus gesehen sechs nächsten Bäume, mit $i=1$..n. An jedem der systematisch (AKÇA 2001, S. 41 f.) über die Bestandesfläche verteilten $n$ Stichprobenpunkte werden die Art, der Brusthöhendurchmesser und gegebenenfalls weitere Attribute der Probebäume erhoben. Der Abstand vom Stichprobenpunkt zur Stammachse des sechstnächsten Baumes liefert den Radius $r_{i}$ des ideellen Probekreises, auf dessen Basis die Schätzung der flächenbezogenen Zielvariablen erfolgt. Allerdings ist auch von der 6-Baum-Stichprobe bekannt, daß die Schätzung der Stammzahl und Grundfläche pro ha insbesondere bei nicht-zufälligen Baumverteilungsmustern verzerrte Ergebnisse liefert. JONSSON et al. (1992) haben gezeigt, daß das Vorzeichen des systematischen Fehlers (Bias) bei regelmäßiger Verteilung negativ und bei geklumpter Verteilung positiv ist, und daß der Fehlerbetrag mit zunehmender Abweichung von der $\mathrm{Zu}$ fallsverteilung zunimmt.

Aus diesem Grund wurde mit dem punktbezogenen Winkelmaß $\boldsymbol{W}_{\boldsymbol{P}}$ (STAUPENDAHL und ZUCCHINI 2006) ein auf dem Ansatz von GADOW et al. (1998) beruhender Aggregationsindex entwickelt, der in Kombination mit der 6-Baum-Stichprobe schnell und einfach zu bestimmen ist und eine enge Korrelation zur Richtung und zum Ausmaß der Verzerrung zeigt (STAUPENDAHL 2008). Ein Regressionsmodell, das diese Korrelation quantifiziert, wurde als bestandesindividuell zu bestimmender Korrekturfaktor in den Stammzahlschätzer integriert, wodurch der Bias auf ein tolerables Maß reduziert wird. Vermeidet man durch eine getrennte Erfassung der Bestandesschichten, daß durch die 6-Baumstichprobe Bäume mit sich überlagernden Standflächen erfaßt werden, hält sich auch der Bias des Schätzers für die mittlere Baumgrundfläche und damit für die Grundfläche pro ha (als Produkt aus Stammzahl pro ha und mittlerer Baumgrundfläche) in vertretbaren Grenzen (STAUPENDAHL 2008; vgl. a. HIRNER 1978, S. 77 f.). 
Die sich aus diesem Vorgehen ergebende Stratifizierung nach Bestandesschichten ${ }^{6}$ empfiehlt sich auch aus anderen Gründen: In den häufig anzutreffenden zweischichtigen $\mathrm{Bu}-$ chenbeständen mit stammzahlreichem Unterstand würden z. B. ohne Stratifizierung sehr viele unterständige Buchen erfaßt, während die wertvollen Stämme des Hauptbestandes nur mit geringer Stammzahl in die Stichprobe eingingen. Damit würden der Unterstand zu genau und der Hauptbestand zu ungenau geschätzt. Auf der Ebene von permanenten Probekreisinventuren führten diese Überlegungen zur Einführung konzentrischer Probekreise (vgl. AKÇA 2001 S. 39). Die Konsequenzen für die 6-Baum-Stichprobe sind in Abb. 7 dargestellt. Demnach werden bei der Auswahl der sechs nächsten Bäume im Rahmen der modifizierten 6-BaumStichprobe nur die Bäume des Hauptbestandes berücksichtigt.

An einem Stichprobenpunkt $i$ evtl. vorkommender Überhalt oder Unterstand (Schichten des Nebenbestandes) wird dagegen durch eine okulare Schätzung der Stammzahl $\left(n_{N B, i}\right)$ und des Durchmessers des Grundflächenmittelstamms $\left(d g_{N B, i}\right)$ innerhalb eines festen Radius $\left(r_{N B, i}\right)$ von $6 \mathbf{~ m}$ (im Unterstand) bzw. 15 m (im Überhalt) erfaßt. Dabei wird davon ausgegangen, daß an einem einzelnen Stichprobenpunkt zusätzlich zum Hauptbestand nur eine dieser beiden Schichten vorkommt. In Beständen ohne eindeutig abgrenzbare Schichten mit exponentiell abnehmender Durchmesserverteilung (z. B. in Plenterwäldern) sollte durch Festlegung eines Grenzdurchmessers ein Hauptbestand und Unterstand definiert werden, wobei dieser Durchmesser so zu wählen wäre, daß beide ,Schichten“ möglichst gut getrennt werden, um die Zahl der Bäume zu minimieren, bei denen unklar ist, zu welchem Kollektiv sie gehören.

Ein weiterer wichtiger Aspekt ist die Begrenzung des Probekreisradius, der bei der 6Baum-Stichprobe normalerweise durch den Abstand des sechstnächsten Baumes zum Probekreismittelpunkt definiert ist. HIRNER (1978, S. 70) wies bereits darauf hin, daß in schwach bestockten Bestandespartien und auf Blößen die Durchführung der 6-Baum-Stichprobe sehr erschwert wird, da aufgrund der dort großen Meßdistanzen die Ermittlung des 6. Baumes sehr zeitaufwendig ist, wodurch der Vorteil des Verfahrens, nämlich die einfache und rasche Merkmalserhebung, wieder hinfällig wird.

\footnotetext{
${ }^{6}$ Nach der niedersächsischen Forsteinrichtungsanweisung (NIEDERSÄCHSISCHE LANDESFORSTEN 1987) sind die Bestandesschichten folgendermaßen definiert: Hauptbestand: Bestandesschicht, auf der das wirtschaftliche Hauptgewicht liegt; Überhalt: Reste aus dem Vorbestand über dem Hauptbestand mit einem Bestockungsgrad $\leq 0,3$; Unterstand: Unter dem geschlossenen Hauptbestand wachsender Baum- und Strauchwuchs, [...].
} 


\section{6-Baum-Stichprobe}

- Probekreisradius = Abstand vom Stichprobenpunkt zum Zentrum des sechstnächsten Baumes

- Maximalradius $=\mathbf{1 5} \mathbf{~ m}$ !

- Datenerhebung: BHD, Arten, Klumpung

\section{Okulare Schätzung}

- Probekreisradius: 6 m im Unterstand und $15 \mathrm{~m}$ im Überhalt

- Okulare Schätzung der Stammzahl und des Dg, getrennt nach Arten

\section{Verjüngungsinventur}

- Probekreisradius: $1,78 \mathrm{~m}$

- Auszählen der Pflanzenzahl

- Bestimmen der Baumart, Höhe und Verbißbelastung der erstnächsten Pflanze

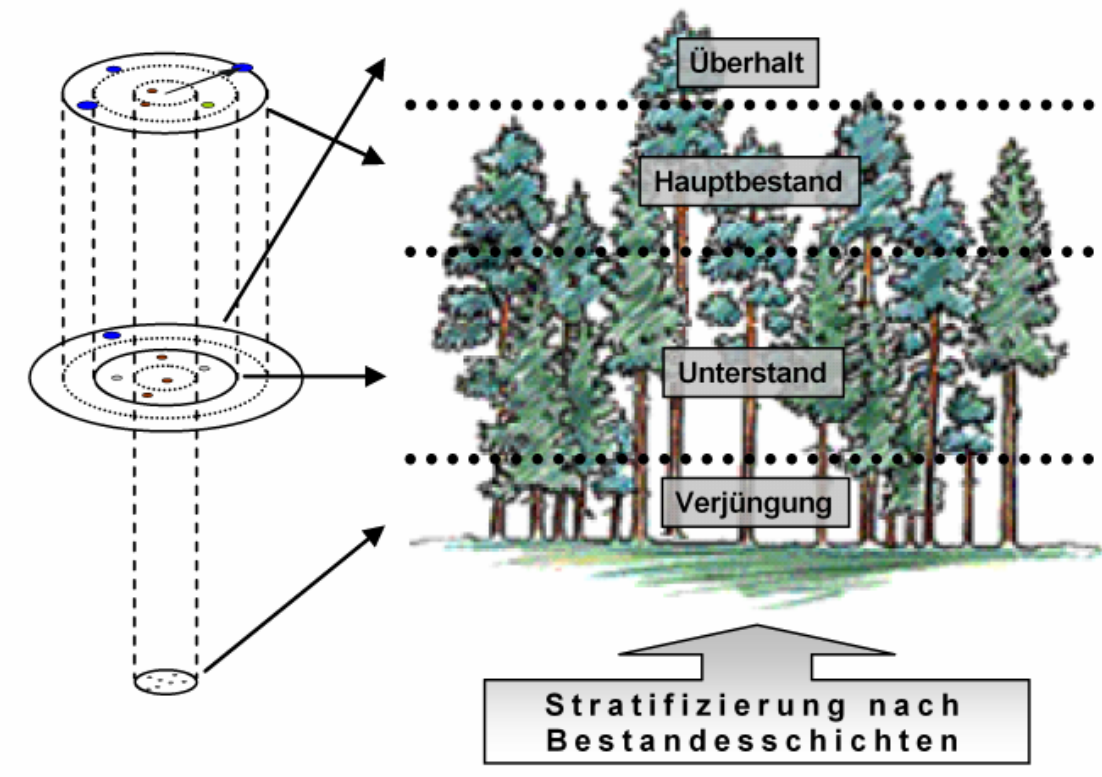

Abb. 7. Schematische Darstellung der Stratifizierung eines Bestandes nach Schichten und der sich daraus ergebenden Inventurarbeiten am Stichprobenpunkt. Um das Verfahren in seiner Vollständigkeit demonstrieren zu können, ist hier ein Fall konstruiert, in dem im Nebenbestand Unterstand und Überhalt gleichzeitig vorkommen. Dies kann für die Praxis ausgeschlossen werden, so daß nie mehr als maximal drei Probekreise aufzunehmen sind (zur Stratifizierung in Plenterbeständen s. Anm. im Text).

Schematic diagram showing the stratification of a forest stand by stand layers and illustration of the field work that has to be accomplished at each sample point. In order to demonstrate the complete method, both the understory and old trees remaining from the former stand are shown together; a situation, which practically never occurs. Thus, at most three sample plots have to be measured (for stratification in plenter stands, see ann. in the text).

Deshalb werden in der modifizierten 6-Baum-Stichprobe nur diejenigen Bäume des Hauptbestandes berücksichtigt, die maximal 15 m vom Stichprobenpunkt entfernt sind. In Bereichen mit geringer Bestandesdichte findet also ein Wechsel von Probekreisen mit variablem Radius und fester Stammzahl $\left(n_{i}=6\right)$ zu Probekreisen mit festem Radius und variabler Stammzahl statt $\left(0 \leq n_{i} \leq 5\right)^{7}$. In beiden Fällen werden die flächenbezogenen Schätzgrößen zunächst für jeden Probekreis auf Hektar-Werte hochgerechnet und anschließend über alle Probekreise arithmetisch gemittelt. Ein systematischer Fehler der Stammzahlschätzung ist nur für die Probekreise mit variablem Radius zu erwarten, so daß das oben beschriebene Korrekturverfahren auch nur auf diesen Teil der Stichprobe angewendet wird.

Wenn $d_{i j}$ die Brusthöhendurchmesser der Bäume des $i$-ten Probekreises der 6-BaumStichprobe bezeichnet, mit $j=1 . . n_{i}$, dann lauten die Schätzer für die Stammzahl und Grundfläche pro ha (STAUPENDAHL 2008):

\footnotetext{
${ }^{7}$ Bei größeren Blößen ist es natürlich sinnvoll, diese vor der Inventur (z. B. Mit Hilfe eines Luftbildes) zu ermitteln und bei der Stichprobennahme unberücksichtigt zu lassen. Dies beschleunigt die Meßarbeiten erheblich und kann den Stichprobenfehler deutlich reduzieren
} 


$$
\begin{aligned}
\hat{N}_{h a}=\frac{10000}{\pi \cdot n} \cdot \sum_{\mathrm{i}=1}^{\mathrm{n}} \frac{n_{i}^{*}}{r_{i}^{2}}+\frac{n_{N B, i}}{r_{N B, i}^{2}} \text { und } \\
\hat{G}_{h a}=\frac{10000}{\pi \cdot n} \cdot \sum_{\mathrm{i}=1}^{\mathrm{n}} \frac{n_{i}^{*}}{r_{i}^{2}} \cdot \bar{g}_{i}+\frac{n_{N B, i}}{r_{N B, i}^{2}} \cdot \bar{g}_{N B, i}, \\
\text { mit } \quad n_{i}^{*}= \begin{cases}n_{i} & \text { falls } n_{i}<6 \\
\frac{0,9246+1,3586 \cdot \hat{W}_{P}^{3,1094}}{n_{i}} & \text { falls } n_{i}=6\end{cases} \\
\bar{g}_{i}=\frac{\pi}{40000 \cdot n_{i}} \cdot \sum_{j=1}^{n_{i}} d_{i j}^{2} \\
\bar{g}_{N B, i}=\frac{\pi}{40000} \cdot d g_{N B, i}^{2}
\end{aligned}
$$

Der durch die okulare Schätzung im Nebenbestand zusätzlich entstehende Fehler, sowohl zufälliger als auch systematischer Art, dürfte nach einiger Übung in vertretbaren Grenzen zu halten sein, zumal die absolute und/oder relative Höhe der Grundfläche (als i.d.R. maßgebliche meßbare Variable einer Bestandesinventur) im Nebenbestand fast immer sehr gering ist ${ }^{8}$. Dafür wird der Hauptbestand, der den Hauptanteil des Bestandesvorrates bildet, um so genauer geschätzt, so daß der Fehler der Vorratsschätzung des Gesamtbestandes je nach Bestandesstruktur z. T. erheblich geringer ist als bei der Variante ohne Stratifizierung (vgl. STAUPENDAHL 2008).

Der Fehler, der durch Probekreise entsteht, die den Bestandesrand schneiden, wird mit Hilfe der Methode von BEERs (1966) korrigiert. Hierbei werden nur diejenigen Probekreise aufgenommen, deren Zentrum innerhalb der Bestandesfläche liegt. Der Radius der RandProbekreise bleibt unverändert, zusätzlich wird jedoch der kürzeste Abstand $a$ des Probekreismittelpunkts zum Bestandesrand gemessen. Über diesen Abstand und den Probekreisradius $r$ läßt sich der Korrekturfaktor $k$ berechnen, mit $0 \leq k \leq 2$ und $k=0$ für $a \geq r$ und $k=2$ für $a=0$. Bei der Auswertung wird den Rand-Probekreisen mit diesem Faktor ein erhöhtes Gewicht zugewiesen, wodurch die geringere Auswahlwahrscheinlichkeit der Randbäume kompensiert wird. Da dieses Verfahren besonders einfach ist, wenig zusätzlichen Meßaufwand erfordert und den systematischen Randfehler fast vollständig eliminiert, wird es auch von SCHMID-HAAS (1969b) empfohlen.

Die Höhenmessung ist die zeitaufwendigste Arbeit bei der Datenerhebung. Um dennoch für jeden Baum das Stamm- bzw. Derbholzvolumen berechnen zu können (NAGEL 1988), werden die Höhen der Einzelbäume getrennt nach Bestandesschichten mit Hilfe einer Ein-

\footnotetext{
${ }^{8}$ Zur Kalibrierung und Kontrolle der Schätzung sollten allerdings in regelmäßigen Abständen einige Bäume gekluppt und Grenzstämme durch Abstandsmessung kontrolliert werden.
} 
heitshöhenkurve geschätzt (KRAMER und AKÇA 1995, S. 138). Die als Eingangsgröße hierfür benötigte Mittelhöhe sollte für jede Hauptbaumart des Hauptbestandes über ca. 5 Höhenmessungen an Bäumen mit einem BHD im Bereich des Mitteldurchmessers (des Durchmessers des Grundflächenmittelstamms) ermittelt werden. Für selten vorkommende Arten des Hauptbestandes sowie für die Baumarten des Nebenbestandes sollte eine gutachtliche Schätzung der Mittelhöhe ausreichend sein.

Desweiteren sollte eine Bestandesinventur auch den Verjüngungszustand erfassen, zumal ein großer Anteil der Arbeitszeit allein auf das Aufsuchen der Stichprobenpunkte entfällt und zuverlässige Informationen über die Verjüngung in anspruchsvollen Waldbaukonzepten von besonderer Bedeutung sind. Es erscheint also sinnvoll, an jedem Stichprobenpunkt möglichst vielfältige Informationen zu erheben. Da das Arbeitsvolumen aber in einem vertretbaren Rahmen bleiben muß, wird für die Erfassung der Verjüngungssituation das Verfahren nach STAUPENDAHL (1997) vorgeschlagen, das besonders einfach und schnell anzuwenden ist. Tab. 1 zeigt eine Auflistung aller an einem Stichprobenpunkt zu erhebenden Variablen.

Tab. 1. Übersicht über die am Stichprobenpunkt - getrennt nach Bestandesschichten - zu erfassenden Variablen. Höhe und Kronenansatz werden natürlich nicht an jedem Probebaum gemessen (s. Text). Auch der Abstand vom Bestandesrand muß nur dann gemessen werden, wenn der Probekreis die Bestandesgrenze schneidet, und das Winkelmaß ist nur dann zu ermitteln, wenn tatsächlich sechs Bäume im Probekreis der 6-Baum-Stichprobe liegen. Optional können bei den Probebäumen weitere Merkmale (z. B. die Güteklasse des Erdstammstückes, etc.) erfaßt werden.

Summary of the variables which have to be collected at each sample point, listed separately for each stand layer. Needless to say, the tree height and height to crown base are not measured at each sample tree (see text). Even the distance to the stand edge must only be measured if the sample plot is extending beyond the stand boundary. The Winkelmass must be assessed only if the six-tree-sample actually includes six trees. Optional variables of the sample trees are the quality class of the lower stem section, for example.

\begin{tabular}{cccc}
\hline & Probekreis & $\begin{array}{c}\text { Variablen je... } \\
\text { Probebaum }\end{array}$ & Baumart \\
\hline $\begin{array}{c}\text { Hauptbestand } \\
\text { (6-Baum-Stichprobe) }\end{array}$ & $\begin{array}{c}\text { Radius, Winkelmaß, } \\
\text { Abstand vom } \\
\text { Bestandesrand }\end{array}$ & $\begin{array}{c}\text { BHD, Höhe, } \\
\text { Kronenansatz, ... }\end{array}$ & - \\
$\begin{array}{c}\text { Überhalt / Unterstand } \\
\text { (okulare Schätzung) }\end{array}$ & $\begin{array}{c}\text { Abstand vom } \\
\text { Bestandesrand }\end{array}$ & - & $\begin{array}{c}\text { Mitteldurchmesser (Dg), } \\
\text { Mittelhöhe (Hg), } \\
\text { Nachwuchs }\end{array}$ \\
$\begin{array}{c}\text { Pflammzahl } \\
\text { (Verjüngungsinventur) }\end{array}$ & $\begin{array}{c}\text { Pflanzenhöhe, } \\
\text { Verbißklasse }\end{array}$ & - & \\
\hline
\end{tabular}

Die Verwendung eines mobilen Datenerfassungsgerätes (MDE) hat sich bei der Datenerfassung als sehr hilfreich erwiesen. Ist eine geeignete Software installiert, wird der Arbeitsablauf merklich beschleunigt, vor allem dann, wenn die Inventur von nur einer Person durchgeführt wird. Das Tragen eines sperrigen Schreibbrettes erübrigt sich und bei schlechtem Wetter gibt es keine Probleme mit aufgeweichten Formularen und versagenden Schreibgeräten. Da die Daten über eine Schnittstelle auf den PC übertragen werden können, entfällt deren manuelle 
Eingabe, wodurch gleichzeitig Zahlendreher und andere Tippfehler ausgeschaltet werden. Werden im Datenerfassungsprogramm Plausibilitätsroutinen integriert, können zudem Fehler bei der Datenerfassung frühzeitig erkannt und bereits während der Inventurarbeiten im Wald korrigiert werden.

\section{Software zur Verarbeitung und Darstellung der Inventurdaten}

Ein forstliches Informationssystem und dynamisches Betriebswerk erlaubt im Gegensatz zu einem traditionellen Forsteinrichtungswerk, flexibel auf unterschiedliche Informationsbedürfnisse des Forstbetriebes zu reagieren. FÄHSER (1983) weist auf die Notwendigkeit einer dynamischen Informationsbereitstellung hin, wenn eine qualitativ hochwertige Forsteinrichtung bzw. Informationserfassung auch zu einer Leistungssteigerung im Betriebsablauf führen soll (vgl. a. BITTER 1990; KÄTSCH 1998). Bereits bei der Aufbereitung der erhobenen Daten lassen sich Effizienz und Marktorientierung der Inventuren deutlich steigern, wenn ein flexibles und leistungsfähiges Berechnungssystem verwendet wird (PUUMALAINEN et al. 1998). Parallel zur Konzeption eines schlüssigen Inventurdesigns sollte daher der Entwicklung eines anwenderfreundlichen und leistungsfähigen Datenbank- und Auswertungsprogramms besondere Aufmerksamkeit geschenkt werden. Abb. 8 illustriert den grundsätzlichen Aufbau der Forstplanungssoftware ARGUS Waldplaner+, die in der Programmiersprache Object Pascal (Entwicklungsumgebung Borland Delphi) entwickelt wurde und auf die besonderen Anforderungen von Eingriffsinventuren abgestimmt ist.

Zentrales Element dieses Programms ist ein Datensteuerungs- und Auswertungsmodul, das den Datenimport regelt und benutzerdefinierte Abfragen an die Datenbank weiterleitet und auswertet. Mit Hilfe der von NAgel (2005) in der Programmiersprache Java entwikkelten und in das Programm integrierten Softwarekomponente TreeGrOSS (Tree Growth Open Source Software) ${ }^{9}$ werden die Datenergänzung (Generierung von Einzelbaumdaten aus schichten- und artspezifischen Mittelwerten), die Z-Baum-basierte und zielstärkenorientierte Nutzungsschätzung (NAGEL 2004) und die Fortschreibung der Bestandesdaten gesteuert. Die Routinen zur Aktualisierung der Bestandesdaten nach Durchforstungen und Nutzungen werden ebenfalls von diesem Modul aufgerufen. Die Forsteinrichtungsdaten sind in den Tabellen einer Microsoft Access Datenbank gespeichert, auf die das Datensteuerungsmodul über die Datenbankschnittstelle DirectAccess ${ }^{10}$ zugreift.

\footnotetext{
9 http://treegross.sourceforge.net, 27. Juli 2007.

10 http://www.opus.ch/OpenSource/DirectAccess/Default.htm, 27. Juli 2007.
} 


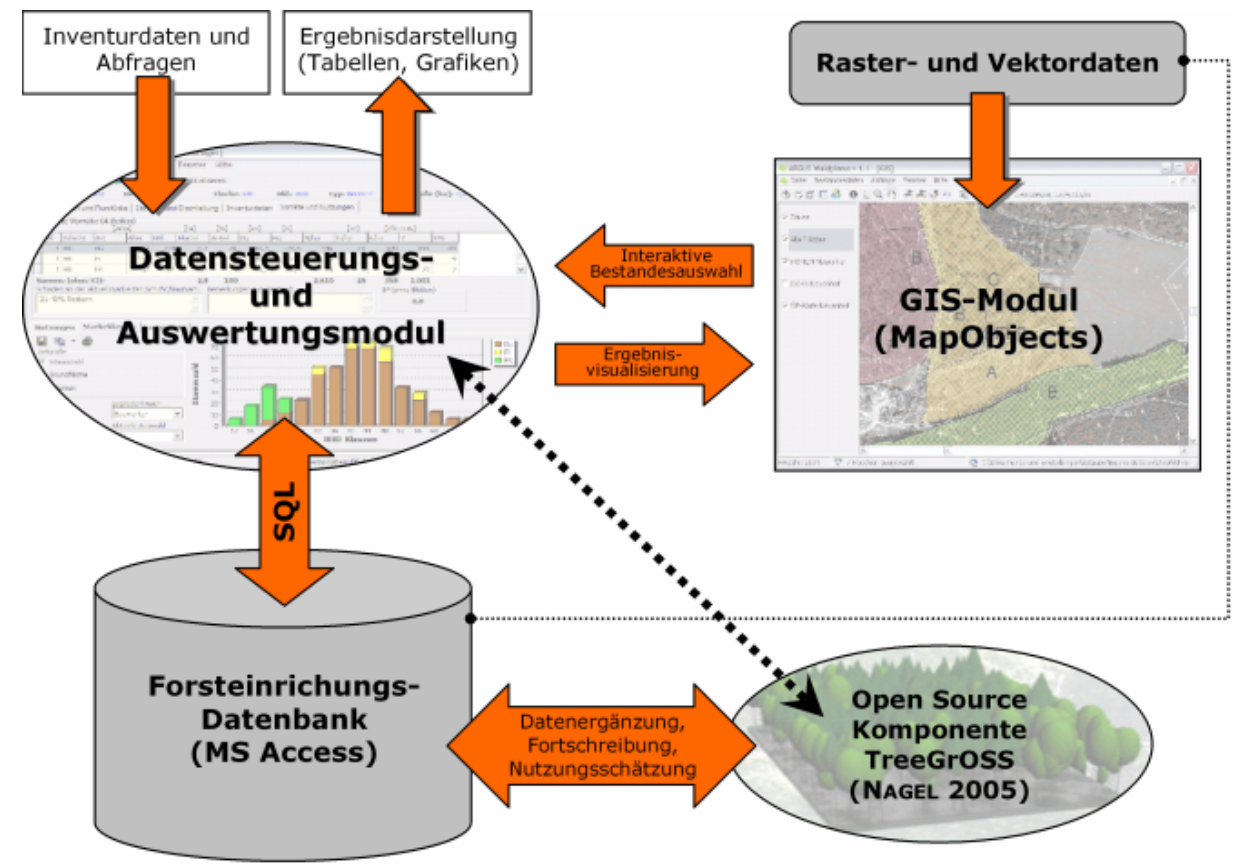

Abb. 8. Struktur des Programms ARGUS Waldplaner+ zur Verarbeitung und Präsentation von Daten aus Eingriffsinventuren. Zentrales Element ist das Programmodul zur Datensteuerung und -auswertung, das durch ein Modul zur Darstellung raumbezogener Daten ergänzt wird. Vektordaten, die die Bestandesgeometrien beschreiben, sind über einen eindeutigen Schlüssel mit den Forsteinrichtungsdaten verknüpft, die wiederum in einer Access Datenbank gespeichert sind. In das Programm ist die Softwarekomponente TreeGrOSS (NAGEL 2005) integriert, mit der die aktuellen Datenergänzungsroutinen und Wuchsmodelle der Nordwestdeutschen Forstlichen Versuchsanstalt zur Verfügung gestellt werden.

General structure of the software ARGUS Waldplaner+, which is used to process and present the data from harvest event assessments. The Kernel of the program is a module for data processing and analysis, which is complemented by a module for the presentation of GIS data. Vector data, which represents the stand geometries are linked to the forest management data by a unique key. The forest management data are stored in an Access database. The software component TreeGrOSS (NAGEL 2005) is used to amend missing data and implement the growth models of the Forest Research Station of Northwestern Germany.

Daten aus diesen Tabellen können mit einem speziellen Abfrage-Dialog selektiert werden, der neben der visuellen und komfortablen Erzeugung komplexer SQL-Abfragen auch deren Speicherung und Wiederverwendung, sowie den Export der selektierten Datensätze für die Verwendung in externen Programmen erlaubt. Die Inventurdaten werden entweder über die serielle oder USB-Schnittstelle direkt aus dem Speicher des MDE-Gerätes oder - wenn die Dateneingabe manuell erfolgte - aus einer ASCII-Datei eingelesen. Da die Auflösung der (gemessenen oder geschätzten) Daten bis zur Einzelbaumebene reicht, sind entsprechend differenzierte Abfragen möglich. In Verbindung mit den von SCHMIDT (2001) entwickelten Modellgleichungen, die eine kombinierte Stärke- und Güteklassensortierung auf der Ebene des Einzelbaumes erlauben, ist es z. B. möglich, abzuschätzen, wo und in und welchen Mengen ein bestimmtes Sortiment innerhalb des Forstbetriebes zu erwarten ist (vgl. a. SCHMIDT 2004). Entsprechende Kundenanfragen können auf diese Weise schnell beantwortet werden.

Ein zweites Programmodul erlaubt die kartographische Darstellung der interessierenden Bestände und die Visualisierung der Datenbankabfragen. Vor dem Hintergrund des räumlichen Bezugs der meisten Planungsprozesse und betrieblichen Maßnahmen ist der Nutzen 
Geographischer Informationssysteme (GIS) in der Forstwirtschaft unbestritten (z. B. BITTER 1990; SPORS et al. 1992; TeUfFEL und KREBS 1996). Aufgrund des räumlichen Charakters von Beständen und ihrer Bedeutung als Planungs-, Kontroll- und Buchungseinheit drängt sich die Verwendung eines GIS - natürlich besonders bei einem bestandesbezogenen Planungsansatz - geradezu auf. Das in Waldplaner+ integrierte GIS-Modul wurde mit Hilfe der MapObjects-Komponenten der Firma ESRI programmiert und ermöglicht den Zugriff auf die gängigsten Vektor- und Rasterdatenformate. Durch die Integration dieser GIS-Funktionalität ist für den Anwender die zusätzliche Installation einer eigenen GIS-Software nicht erforderlich. Zudem kann das System flexibel an individuelle Anforderungen angepaßt werden.

Das GIS-Modul dient als „Daten-Viewer“ v. a. der interaktiven und raumbezogenen Selektion von Beständen und Bestandesklassen und der Visualisierung von Abfragen und Auswertungen. Die erzeugten Kartenansichten können ausgedruckt oder in eine Grafikdatei gespeichert und in anderen Programmen weiterverwendet werden. Ein Beispiel wäre die Erzeugung einer Karte mit allen Beständen, deren Bestockungsgrad einen bestimmten kritischen Wert überschritten hat, um einen Überblick über die Anzahl, Gesamtfläche und Verteilung der besonders pflegebedürftigen Waldteile zu bekommen. Auf der Grundlage dieser Funktionalität kann der Waldplaner+ zu einem Werkzeug zur Unterstützung von Entscheidungsprozessen (Decision Support System) ausgebaut werden (vgl. KÄTSCH 1998; VACIK und LEXER 2001; SODTKE et al. 2005; NAGEL und SCHMIDT 2006). Gleichzeitig steht durch die Kompatibilität der verwendeten Datenformate die gesamte Funktionalität von Microsoft Access und der GIS-Programme ARC/INFO und ArcView zur Verfügung, so daß die Daten auf vielfältigste Art und Weise bearbeitet und analysiert werden können.

\section{Résumé}

Das hier vorgestellte Inventurkonzept und seine softwareseitige Umsetzung zielen darauf ab, dem Forstbetrieb möglichst exakt die Daten zur Verfügung zu stellen, die die operative Planung und Entscheidungsfindung bestmöglich unterstützen, um auf diese Weise zur Optimierung des Betriebsergebnisses beizutragen ${ }^{11}$. Da bei einer stratenorientierten Planung Informationen verlorengehen, die bezüglich dieser Entscheidungsprozesse für essentiell gehalten werden, wird im Gegensatz zum Konzept der Betriebsinventur am Bestand als primäre Bezugsebene für Inventur und Planung festgehalten ${ }^{12}$. Zur Reduktion der dadurch entstehenden Ko-

\footnotetext{
11 „Die operative Planung [...] dient auch zur laufenden Ausschöpfung und Anpassung der vorhandenen Leistungspotenziale. Dabei handelt es sich beispielsweise um spezielle Anpassungen des Produktionsprogramms mit dem Ziel, eine möglichst wirtschaftliche Nutzung der Ressourcen zu erreichen.“

(http://de.wikipedia.org/wiki/Operative Planung, 26. Juli 2007)

12 Auch in Betrieben, die mit der Kontrollstichprobe inventarisiert werden, werden in aller Regel (allein schon aus der Notwendigkeit heraus, Betriebskarten anzufertigen) Bestände ausgeschieden, deren Vorräte und Nutzungen allerdings entweder mittels der Regionalisierung (s. Abschnitt 1) oder vom Forsteinrichter mit Hilfe der Vorinformationen aus der Betriebsinventur geschätzt werden (z. B. BöCKMANN et al. 1998b). Wie genau diese (s. auch nächste Seite)
} 
sten beinhaltet das vorgestellte Konzept eine Reihe von Maßnahmen. Hierzu zählen insbesondere

- die Anpassung der Aufnahmeintensität (des Inventurverfahrens) an den tatsächlichen Informationsbedarf und an die vorhandenen Bestandesstrukturen,

- die Vergrößerung der durchschnittlichen Bestandesgröße auf mindestens 5-10 ha,

- die Fortschreibung des Datenbestandes auf der Basis von Wuchsmodellen und der erfaßten Nutzungsdaten und

- die dadurch ermöglichte Verlängerung des Inventurintervalls und Reduzierung der Kosten der mittelfristigen Betriebsplanung.

Zudem wird durch die Aktualität und den Ortsbezug der Daten deren Nutzwert deutlich erhöht. Dies betrifft insbesondere die Planung des Holzeinschlages und die Vermarktung des Rohholzes, die durch die Erfassung des ausscheidenden Bestandes erheblich effizienter und damit auch profitabler gestaltet werden können (z. B. NIEUWENHUIS et al. 2001). Da diese Datenerhebung nur einen überschaubaren Aufwand erfordert und gleichzeitig die Häufigkeit der Inventur des verbleibenden Bestandes reduziert, stellt sie eine besonders effiziente und damit unverzichtbare Komponente des Gesamtsystems dar.

Um den Nutzen aus diesem Inventursystem weiter zu steigern, sollten die Bestandesdaten in jedem Fall auch in Form eines geographischen Informationssystems (GIS) bereitgestellt werden. Eine weitere Effizienzsteigerung ist zu erwarten, wenn die Nutzung von Vorinformationen (z. B. aus großräumigen Waldinventuren) in das Berechnungssystem integriert wird (PuUmalainen 1998, S. 10 ff.). Das hier vorgestellte Datenmodell bildet aufgrund des bestandesbezogenen Ansatzes außerdem eine ideale Basis für eine auf der Mehrpfadtheorie beruhende Optimierung der gesamtbetrieblichen Planung (z. B. GADOW 2005, 2006).

Aufgrund der variierenden, vom Informationsbedürfnis der jeweils entscheidenden Personen abhängigen Inventurintervalle und -intensitäten liegt es nahe, die Verantwortung für die Erfassung der Bestandesdaten an die Forstbetriebsleitung zu delegieren. BULLINGER (2000) weist darauf hin, daß die Gesellschaft zur Zeit eine Umbruchphase der gesamten Arbeitswelt erlebt und diese Entwicklung besondere Anforderungen an die Arbeitsorganisation der Unternehmen stellt (vgl. a. KLOTZ 2000). Innovative Unternehmen werden sich demnach in Zukunft nicht nur durch innovative Produkte, sonders ebenso durch innovative Organisationsstrukturen auszeichnen. Als wesentliche Merkmale einer innovativen Organisation nennt er flache Hierarchien, dezentrale Entscheidungsstrukturen und eine ausgeprägte Ergebnisverantwortung der einzelnen Mitarbeiter und Arbeitsgruppen.

Schätzungen z. B. im Vergleich zu den Ergebnissen des konventionellen Waldbegangs sind und wie hoch dementsprechend ihr Nutzwert ist, ist jedoch unklar. 
Wenn also der einzelne Forstbetrieb die Verantwortung für das Ergebnis seines Wirtschaftens tragen soll, sollte er auch die Instrumente und Mittel in die Hand bekommen, um diesen Erfolg steuern und messen zu können. Bezogen auf das Produktionsmittel und Warenlager „Wald“ bedeutet dies, daß dessen Zustand und Veränderung von denjenigen gemessen werden sollte, die auf diese Größen durch ihr Wirtschaften und ihre Entscheidungen direkten Einfluß nehmen. In diesem Sinne ist das Konzept der Eingriffsinventur als Instrument zur Selbstkontrolle zu verstehen, das insofern eine Alternative zur nachträglichen, externen Datenerfassung und Kontrolle durch die klassische Forsteinrichtung darstellt. Die Entscheidung, ob die Inventuren durch eigenes Personal oder durch forstliche Dienstleister durchzuführen sind, sollte ebenfalls dem einzelnen Forstbetrieb überlassen werden. Auch hier könnten mit der Einrichtung von evtl. auch forstbetriebsübergreifenden Cost-Centern neue Wege der Arbeitsorganisation beschritten werden (vgl. BULLINGER 2000).

Erste Erfahrungen mit dem hier vorgestellten Konzept in einem rund 1.800 ha großen Privatforstbetrieb zeigen, daß insbesondere die Erfassung der Nutzungen und deren „Verbuchung“ im dynamischen Betriebswerk sorgfältig in die Arbeitsabläufe integriert werden müssen, wenn sie die vorhandenen Arbeitskapazitäten nicht über Gebühr beanspruchen sollen. Dies erfordert auch eine entsprechende Schulung und Einweisung der Betriebsleitung und der mit dem Holzeinschlag beauftragten Mitarbeiter oder Unternehmer. So muß z. B. die korrekte Zuordnung der entnommenen Stämme zum Bestand gewährleistet sein, falls die Daten des ausscheidenden Bestandes durch den Harvester erfaßt werden. Hierbei ist die mit dem Waldplaner+ erstellte Arbeitskarte ein wichtiges Hilfsmittel, mittelfristig sollte jedoch der Einsatz von GIS- und GPS-gestützten Lösungen geprüft werden. In jedem Fall werden zusätzliche Aufgaben nur dann zuverlässig erfüllt werden, wenn ihr (Mehr-)Nutzen erkannt wurde. Dieser muß also auch kommuniziert werden, wodurch deutlich wird, das innovative Organisationsstrukturen entsprechend hohe Anforderungen an Betriebsleitung, Mitarbeiter und Dienstleister stellen.

Die kritische Würdigung der Betriebsergebnisse und die mittelfristige Planung im Rahmen einer 10-jährigen Forsteinrichtung sollte weiterhin wesentlicher Bestandteil der gesamtbetrieblichen Planung und Kontrolle bleiben. Da durch die eingriffsbezogene Datenerfassung und Fortschreibung die aktuellen Zustandsdaten jedes Bestandes jederzeit verfügbar sind, kann hierbei auf messende Arbeiten vollständig verzichtet werden. Dies bedeutet eine erhebliche Einsparung von Mitteln, die freilich mindestens zum Teil den Forstbetrieben für die laufende Aktualisierung der Bestandesdaten zur Verfügung gestellt werden müßten. Darüberhinaus entstehen durch diese Entlastung von Inventuraufgaben Freiräume für eine Fokussierung der Forsteinrichtung auf die strategische Planung, wie sie u. a. von HANEWINKEL (2001) und BACHMANN et al. (2002) gefordert wird (vgl. a. BACHMANN 2005). Die Einbeziehung bestandesbezogener Informationen in den Prozeß der Gesamtplanung stellt hierzu keinen Widerspruch dar (vgl. GADOW 2005). Vielmehr erleichtert die Berücksichtigung der Ge- 
gebenheiten und Potentiale der zu bewirtschaftenden Einheiten - eben der Bestände - den Entwurf eines gesamtbetrieblichen Optimums, das operational ist und somit ein erreichbares Ziel darstellt. Die Methodik und technischen Möglichkeiten zur Umsetzung dieses Ansatzes stehen zur Verfügung.

\section{Danksagung}

Die Autoren danken der Deutschen Forschungsgemeinschaft, die diese Arbeit finanziert hat (Förderprojekt Ga 473/11-2).

\section{Literatur}

AKÇA, A., 2001: Waldinventur. Cuvillier, Göttingen, 193 S.

AKÇA, A., 1993: Zur Methodik und Bedeutung der kontinuierlichen Forstinventuren. Allg. Forst- Jagdztg.164 (11): 193 - 198 .

BachmanN, P., 2005: Forstliche Planung - heute und morgen. Schweiz. Z. Forstwes. 156 (5): 137 - 141.

Bachmann, P., Ammann, S., Kunz, B., Rupf, A., 2002: Moderne forstbetriebliche Planung. Schweiz. Z. Forstwes. 153 (5): 184 - 189.

BEERS, T.W., 1966: The direct correction of boundery-line slopover in horizontal point sampling. Purdue Univ., Agri. Exp. Sta., Lafayette, Ind. Res. Progr. Rep. 224, 8 pp.

BergmanN, A., 1997: Kundenorientierte Rohholzbereitstellung bei vollmechanisierter Holzernte - Ein System für die optimale Einteilung von Sägeabschnitten mit Bordcomputern auf Vollerntern. Diss. Forstl. Fak. Univ. Göttingen, $249 \mathrm{~S}$.

BITTER, A.W., 1990: EDV-gestützte Unternehmensführung im Forstbetrieb mit Hilfe eines flächenbezogenen Betriebsinformationssystems. Diss. Forstl. Fak., Univ. Göttingen.

Böckmann, Th., Saborowski, J., Dahm, S. Nagel, J., und Spellmann, H., 1998a: Die Weiterentwicklung der Betriebsinventur in Niedersachsen. Forst u. Holz 53 (8): 219 - 226.

BöcKmann, Th., Spellmann, H. und HÜSIng, F., 1998b: Neukonzeption und Weiterentwicklung der Forsteinrichtung in den Niedersächsischen Landesforsten. Forst u. Holz 53 (10): 298 - 302.

Bullinger, H.J., 2000: Arbeitsorganisation im Umbruch - Chancen für Mitarbeiter und Betriebe. Forst u. Holz 55: $534-540$.

FÄHSER, L., 1983: Dynamisierung der Forsteinrichtung. AFZ - Der Wald 38 (17): 430 - 432.

Fuchs, A., und KenNEL, E., 1994: Erste Wiederholungsaufnahme einer permanenten Betriebsinventur im bayerischen Staatswald. Forstw. Cbl. 113: 224 - 235.

GADOw, K. v., 2005: Das Mehrpfad-Prinzip der Forsteinrichtung. AFZ - Der Wald 60 (5): 469 - 471.

GADOW, K. v., 2006: Forsteinrichtung - Adaptive Steuerung und Mehrpfadprinzip. Universitätsverlag Göttingen, $163 \mathrm{~S}$.

GADOw, K. V., und CHEN, B.W., 2002: Timber harvest planning with spatial objectives, using the method of simulated annealing. Forstw. Cbl. 121: $25-34$.

Gadow, K. V., Hui, G.Y., und AlBert, M., 1998: Das Winkelmaß - ein Strukturparameter zur Beschreibung der Individualverteilung in Waldbeständen. Centralbl. f. d. ges. Forstw. 115: 1 - 10. 
Gadow, K. v., und PuUmalainen, J., 2000: Scenario planning for sustainable forest management. In: GadOw, K. v., PukKala, T., and Tomé, M., 2000: Sustainable Forest Management. Kluwer Series Managing Forest Ecosystems, Vol. 1: 319 - 356.

Gadow, K. V., und SchmidT, M., 1998: Periodische Inventuren und Eingriffsinventuren. Forst u. Holz 49 (5):129 - 131.

Gadow, K. V., und StüBer, V., 1994: Die Inventuren der Forsteinrichtung. Forst u. Holz 49 (5): 129 - 131.

GAfFrey, D., SABorowski, J., und Spelsberg, G., 1998: Baumartenspezifische Funktionen zur Schätzung von Bestandesdurchmesserverteilungen. . Allg. Forst- u. Jagdztg. 169 (5): 81 - 86.

GÜSSEFELDT, J., 1997: Grundsätzliche Überlegungen zu Regionalisierungsmodellen. Geograph. Zeitschr. 85: 1 19.

Gurnaud, A., 1886: La méthode du contrôle. P. Jacquin, Besancon, 124 S.

Hallenbarter, D., Hasenauer, H., und ZingG, A., 2005: Validierung des Waldwachstumsmodells MOSES für Schweizer Wälder. Schweiz. Z. Forstwes. $156(5)$ : 149 - 156.

HANEWINKEL, M., 2001: Neuausrichtung der Forsteinrichtung als strategisches Managementinstrument. Allg. Forst- u. Jagdztg. 172 (11): 202 - 211.

Hasenauer, H., Moser, M., und EcKMüllner, O., 1995: Ein Programm zur Modellierung von Wachstumsreaktionen. AFZ - Der Wald 50 (4): 216 - 218.

Heinimann, H. R., 1999: Logistik der Holzproduktion - Stand und Entwicklungsperspektiven. Forstwiss. Centralbl. $118(1): 24-38$.

Hinrichs, A., Oesten, G., und Steinbrenner, M., 1993: Die Betriebsinventur der Landesforstverwaltung Baden-Württemberg. Arbeitspapier 14-93. Institut für Forsteinrichtung und Forstliche Betriebswirtschaft, Albert-Ludwigs-Univ. Freiburg, $74 \mathrm{~S}$.

HIRNER, V., 1978: Theoretische Überlegungen zur Sechs-Baum-Stichprobe und deren praktische Anwendungsmöglichkeiten. Univ. Freiburg (Breisgau), Forstwiss. Fak., Diss., 114 S.

HockENJOS, W., 2006: Naturschutzstandards durch Zertifizierungssysteme - Zurück zum Holzacker? AFZ - Der Wald 61 (3): 146 - 151.

Hostettler, M., 2006: Bewirtschaftung des Staatswaldes in einer globalen Wirtschaft. Forst u. Holz 61 (7): $270-273$.

JONSSON, B., HOLM, S., and KALLUR, H., 1992: A forest inventory method based on density-adapted circular plot size. Scand. J. For. Res. 7: $405-421$.

KÄTsCH, C., 1998: Konzeption für ein forstliches Produktionsplanungs- und Steuerungssystem unter besonderer Berücksichtigung der Forsteinrichtung. Schriften aus der Forstl. Fak. der Univ. Göttingen und der Nieders. Forstl. Versuchsanstalt 126. Sauerländer's, Frankfurt a. Main, 211 S.

KASSIER, H., 1980: An integrated system for forest management and silvicultural planing and control. South African Forest Journal 114: 1 - 6.

KLotZ, U., 2000: Neue Unternehmensmodelle führen zu einer anderen Definition von Arbeit. Neue Ökonomie, Teil 3. Frankf. Allg. Zeitung 124, S. 33.

KrAmER, H., und AKÇA, A., 1995: Leitfaden zur Waldmeßlehre. 3. Aufl., J. D. Sauerländer's, Frankfurt a. M., $266 \mathrm{~S}$.

MERKER, K., 2006: Bewirtschaftung des Staatswaldes in einer globalen Wirtschaft. Forst u. Holz 61 (7): $250-252$.

Muinonen, E., Matama, M., Hyppänen, H, and Vainikainen, V., 2001: Forest stand charachteristics estimation using a most similar neigbour approach and image spatial structure information. Remote Sensing of nvironment 78: $223-228$.

NAGEL, J., 1988: Volumenfunktionen. Interner Bericht, Nordwestdeutsche Forstliche Versuchsanstalt, Göttingen. 
NAGEL, J., 1998: Zur Auswertung und Zuwachsprognose von permanenten Stichprobenerhebungen. Forst u. Holz 53 (7): 197 - 198.

NAGEL, J., 1999: Konzeptionelle Überlegungen zum schrittweisen Aufbau eines waldwachstums-kundlichen Simulationssystems für Nordwestdeutschland. Schriften aus der Forstl. Fak. d. Univ. Göttingen und der Nieders. Forstl. Versuchsanst. 128, J. D. Sauerländer’s, Frankf. a. M., 122 S.

NAGEL, J., 2000: Prognose des Zuwachses von Fichten/Buchen-Mischbeständen mit dem Programm BWIN. In: SaborowsKi, J., und SlobodA, B. (Hrsg.): Deutscher Verband Forstlicher Forschungsanstalten, Sektion Forstliche Biometrie und Informatik - 12. Jahrestagung und Internationale Biometrische Gesellschaft Deutsche Region, Arbeitsgruppe Ökologie, Herbstkolloquium, Göttingen, 29. September - 1. Oktober 1999; Die grüne Reihe: 194 - 199.

NAGEL, J., 2004: Nutzungsplanung in Rein- und Mischbeständen. Allg. Forst- u. Jagdztg. 175 (7/8): 150 - 156.

NAGEL, J., 2005: TreeGrOSS - Eine Java basierte Softwarekomponente zur Waldwachstumsmodellierung für Forschung, Lehre und Praxis. In: WunN, U., und QuedenAU, H.D. (Hrsg.): Deutscher Verband Forstlicher Forschungsanstalten Sektion Forstliche Biometrie und Informatik, 15. Tagung Freiburg 9.-10. Oktober 2003, Grüne Reihe: $33-37$

NAGEL, J., und Biging, G.S., 1995: Schätzung der Parameter der Weibullfunktion zur Generierung von Durchmesserverteilungen. Allg. Forst- u. Jagdzeitg.166 (9/10): 185 - 189.

NAGEl, J., and SchMidT, M., 2006: The Silvicultural Decision Support System BWINPro. In: HASENAUER, H. (Ed.): Sustainable Forest Management, Growth Models For Europe, Springer, Berlin, Heidelberg: $59-63$.

Nicke, A., ERTEld, Th., KÖHLeR, B., und TAutKus, M., 2005: Inventur in strukturreichen Beständen. AFZ Der Wald 60 (5): $245-247$.

NIEDERSÄCHSISCHE LANDESFORSTEN, 1987: Anweisung zur Betriebsregelung (Forsteinrichtung) in den Niedersächsischen Landesforsten. Nieders. Minist. f. Ernähr., Landwirtsch. u. Forsten, 55 S.

Nieschulze, J., BöcKmanN, Th., NAGel, J., und SABOROwski, J., 2005: Herleitung von einzelbestandesweisen Informationen aus Betriebsinventuren für die Zwecke der Forsteinrichtung. Allg. Forst- u. Jagdztg. 176 (9/10): $169-176$.

Nieuwenhuis, M., McHugh, F., Malone, L., and Layton, T., 2001: Development and evaluation of preharvest inventory and cross-cutting simulation procedures to maximise value recovery. Irish Forestry 56 (1): $12-28$.

Отто, H.-J., 1992: Langfristige ökologische Waldentwicklung: Ökologische Grundlagen des Regierungsprogramms. AFZ 47 (11): 566 - 568.

POMMERENING, A., 1998: Fortschreibung von Stichprobendaten mit positionsabhängigen Wuchsmodellen. Vortrag anläßlich der Jahrestagung 1998 der Sektion Ertragskunde des Deutschen Verbandes Forstlicher Forschungsanstalten, Tagungsband: $35-51$.

Pommerening, A., und SchmidT, M., 1998: Modifizierung des Stammabstandsverfahrens zur Verbesserung der Stammzahl- und Grundflächenschätzung. Forstarchiv 69: 47 - 53.

Pretzsch, H., 1995: Zum Einfluß des Baumverteilungsmusters auf den Bestandeszuwachs. Allg. Forst- u. Jagdztg. 166: $190-201$.

PRETzSCH, H., 1997: Analysis and modelling of spatial stand structures. Methodological considerations based on mixed beech-larch stands in Lower Saxony. Forest Ecology and Management Vol. 97: 237 - 253.

Pretzsch, H., und KAHN, M., 1996: Wuchsmodelle für die Unterstützung der Wirtschaftsplanung im Forstbetrieb. AFZ - Der Wald 51 (25): 1414 - 1419.

PretzSCH, H., und DURSKY, J., 2001: Evaluierung von Waldwachstumssimulatoren auf Baum- und Bestandesebene. Allg. Forst- u. Jagdztg. 172 (8/9): $146-150$.

ProdAn, M., 1968: Punktstichprobe für die Forsteinrichtung. Der Forst- u. Holzwirt 23: 225 - 226.

PuUmalainen, J., 1998: Marktorientierte Vorratschätzung und terrestrische Vorinformationen in kleinräumigen Waldinventuren. Diss., Fak. f. Forstwissensch., Univ. Göttingen, Cuvillier Verlag Göttingen, 176 S. 
PuUmalainen, J., Gadow, K. v, und KoRHONEn, K., 1998: Flexible Ergebnisberechnung einer Eingriffsinventur. Forstarchiv 69: $145-150$.

RHEINLAND-PfälZISCHES Forstamt DAUN, 2000: Aufnahme des ausscheidenden Bestandes mit verfahrensbezogener Sorten-, Kosten- und Ergebnisschätzung. AFZ - Der Wald 55 (18-19): 997 - 998.

SAGL, W., 1995: Forsteinrichtung auf dem Prüfstand. Schriftenr. Inst. forstl. Betriebswirtsch. u. Forstwirtschaftspolitik d. Univ. f. Bodenkultur Wien Nr. 26, 137 S.

SCHMID-HAAS, P., 1969a: Kontroll-Stichproben: Aufnahmeinstruktion. Mitt. der Eidgen. Anstalt. Forstl. Vers. 27.

Schmid-HAAS, P., 1969b: Stichproben am Waldrand. Mitt. der Eidgen. Anstalt. Forstl. Vers. 45: 233 - 303.

SchMID-HAAS, P., 1989: Schweizer Kontrollstichprobenverfahren in der Forsteinrichtung. Schweiz. Z. Forstwes. 140: 43 - 56.

SCHMIDT, M., 2001: Prognosemodelle für ausgewählte Holzqualitätsmerkmale wichtiger Baumarten. Diss., Fak. F. Forstwiss., Univ. Göttingen, $302 \mathrm{~S}$.

SCHMIDT, M., 2004: Simulative Astigkeits- und Qualitätsprognose für Douglasien-Rundholz. Allg. Forst- u. Jagdztg. 175: $49-60$.

SCHOBER, R., 1995: Ertragstafeln wichtiger Baumarten. 4. Aufl., Sauerländer, Frankfurt a. M.

SCHÖNSLEBEN, P., 2000: Integrales Logistikmanagement : Planung und Steuerung von umfassenden Geschäftsprozessen. 2., überarb. und erw. Aufl., Springer, Berlin, 778 S.

SCHÖPFER, W., 1998: Schritte zu einem Informationssystem für Holzernte und Holzvermarktung. Forst und Holz 53 (12): $379-387$.

Sodtke, R., Schmidt, M., Fabrika, M., Nagel, J., Dursky, J., und Pretzsch, H., 2004 : Anwendung und Einsatz von Einzelbaummodellen als Komponenten von entscheidungsunterstützenden Systemen für die strategische Forstbetriebsplanung. Forstarchiv (75) 51-64.

SPEIDEL, G., 1972: Planung im Forstbetrieb. Parey, Hamburg und Berlin, 276 S.

SPELlmANn, H., und AKৎA, A., 1983: Verbesserungsmöglichkeiten in der Forsteinrichtung. AFZ - Der Wald 38 (17): 427 - 430.

Spellmann, H., Nagel, J., und BöcKmann, TH., 1999: Summarische Nutzungsplanung auf der Basis von Betriebsinventuren. Allg. Forst- u. Jagdztg.170 (7): $122-128$.

SpoRs, H.-J., Stock, R., und Sloboda, B., 1992: Räumliche Informationssysteme als Entscheidungsbasis für die forstliche Praxis. Forstarchiv 63: 33 - 39.

STAUPENDAHL, K., 1997: Ein neues Stichprobenverfahren zur Erfassung und Beschreibung von Naturverjüngung. In: Pelz, D.R. (Hrsg.): Deutscher Verband Forstlicher Forschungsanstalten, Sektion Forstliche Biometrie und Informatik - 10. Jahrestagung, Freiburg, 24. - 26. September 1997, IUFRO, Grüne Reihe: $32-49$.

StAUPENDAHL, K., 1999: Modelling thinnings based on the ratio of relative removal rates. In: PUKKaLA, T., and EERIKÄINEN, K. (eds.): Growth and yield modelling of tree plantations in South and East Africa - Proceedings of the meeting in Mombasa, Kenya, 12.-15. Oct. 1999. The Univ. of Joensuu, Faculty of Forestry Res. Notes 97: 183 - 194.

Staupendahl, K., 2008: Die modifizierte 6-Baum-Stichprobe - Ein geeignetes Verfahren zur Erfassung von Waldbeständen. Allg. Forst- u. Jagdztg. 179 (im Druck).

Staupendahl, K., und PuUmalainen, J., 1999: Modellierung des Einflusses von Durchforstungen auf die Durchmesserverteilung von gleichaltrigen Fichtenreinbeständen. Centralbl. f. d. ges. Forstw. 116 (4): $249-262$.

STAUPENDAHL, K., and ZUCCHINI, W., 2006: Estimating the spatial distribution in forest stands by counting small angles between nearest neighbours. Allg. Forst- u. Jagdztg. 177 (8/9): 160 - 168.

STERBA, H., 1990: Validitätsprüfung des Einzelbaumsimulators für Fichten-Kiefern-Mischbestände. Tagungsbericht, Deutscher Verb. Forstl. Forschungsanstalten - Sektion Ertragskunde: 101 - 117. 
STÜMER, W., 2004: Kombination von terrestrischen Aufnahmen und Fernerkundungsdaten mit Hilfe der kNNMethode zur Klassifizierung und Kartierung von Wäldern. Diss., Fak. Forst-, Geo- und Hydrowiss., Techn. Univ. Dresden, 153 S.

TeUfFel, K. Frhr. V., und KREBS, M. 1996: Geoinformationssysteme als Instrumente der forstlichen Betriebsführung. AFZ - Der Wald 10: 532 - 542.

Teuffel, K. FrHR. V., und KreBS, M. 1999:Forsteinrichtung im Wandel. AFZ - Der Wald 54: 858 - 865.

TzSChUPKE, W., 1991: Betriebs- oder bestandesbezogene Waldzustandsinventuren für die Forsteinrichtung. AFZ - Der Wald162 (10): 195 - 200.

UUSITALO, J., 1995: Pre-harvest measurement of pine stands for sawing production planning. University of Helsinki, Department of Forest Resource Management, Publications 9, 96 p.

UUSitAlo, J., and KivineN, V.P., 2000: EMO: A pre-harvest measurement tool for predicting forest composition. In: UsEnIUS, A. et al. (eds.): Proc. of 3rd workshop on measuring of wood properties, grades and qualities in the conversion chains and global wood chain optimisation in Espoo, Finland: 173 - 180.

VACIK, H, and LEXER, M., 2001: Application of a spatial decision support system in managing the protection forests of Vienna for sustained yield of water resources. For. Ecol. Managem. 128: 1 -2, 49 - 55.

WALTER, F., and CARLSSON, D., 1998: New system for optimizing transport. SkogForsk News 1: p. 1.

WEIDENBACH, P., und KARIUS, K., 1993: Betriebsinventur auf Stichprobenbasis als Element moderner Forsteinrichtung. AFZ - Der Wald 48 (13): 685 - 688. 



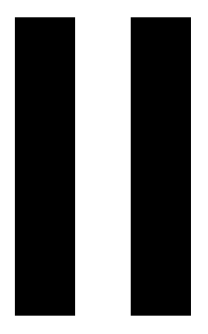





\title{
Die modifizierte 6-Baum-Stichprobe - ein geeignetes Verfahren zur Erfassung von Waldbeständen
}

\author{
The modified six-tree-sample - a suitable method for forest stand assessment
}

\author{
KAI STAUPENDAHL
}

Allgemeine Forst- und Jagdzeitung 179 (im Druck)

Schlagwörter: Abstandsverfahren; 6-Baum-Stichprobe; Bias; räumliche Verteilung; Bestandesinventur.

\section{Zusammenfassung}

Die von Prodan vorgeschlagene 6-Baum-Stichprobe gilt als einfach und schnell anzuwendendes Verfahren der Bestandesinventur. Ein Problem war bisher allerdings der nicht kalkulierbare, methodisch bedingte Bias bei der Schätzung der Stammzahl und Grundfläche pro Hektar in Beständen mit nicht-zufälligem Verteilungsmuster. Der hier vorgestellte Ansatz verwendet zur Korrektur des Stammzahlbias einen variablen Faktor, dessen bestandesindividueller Wert durch einen einfach zu erhebenden Aggregationsindex bestimmt wird. Zur Verminderung des Bias der Grundflächenschätzung und zur weiteren Verbesserung der Praktikabilität des Verfahrens werden zudem aufnahmetechnische Modifikationen vorgeschlagen. Die Validierung mit Hilfe von Stichprobensimulationen zeigt, daß die vorgeschlagenen Änderungen die Effizienz der 6-Baum-Stichprobe deutlich erhöhen und deren Anwendbarkeit in Beständen beliebiger Struktur ermöglichen. Zur weiteren Absicherung dieser Ergebnisse wären Zeitstudien und Simulationen zur Auswirkung von systematischen Fehlern, die aufgrund von Modifikationen bei der Erfassung des Nebenbestandes auftreten könnten, wünschenswert.

Key words: Distance methods; six-tree-sample; bias; spatial patterns; stand inventory.

\begin{abstract}
The "six-tree-sample" method, proposed by Prodan for stand assessment, is cost-effective and easy to apply in the field. If the spatial tree distribution is not random, however the estimation of the stem number and basal area per hectare is biased. The approach presented here corrects the bias of the stem number using a variable factor, whose stand individual value depends on an aggregation index, which can be determined with marginal additional effort. In order to reduce the bias of the basal area and to further improve the practicability of the method, modifications of the data collection technique are proposed. The validation by means of sampling
\end{abstract}


simulations shows, that the proposed changes both increase the efficiency of the six-treesample significantly and allow its application in stands with any spatial pattern. Time studies and further simulation studies would be eligible, in order to analyse systematic errors, which could occur due to the modified data collection technique for the assessment of the understory.

\section{$1 \quad$ Einleitung}

Seit KÖNIG (1835, S. 388) den Abstand zwischen Bäumen zur Schätzung der Stammzahl pro Hektar nutzte, sind die sogenannten Abstandsverfahren Gegenstand zahlreicher Untersuchungen gwesen (vgl. LOETSCH et al. 1973, S. 369 ff.; PICARD et al. 2005; KLEINN und VILČKO 2006a, 2006b). Bei dieser Gruppe von Inventurmethoden werden entweder die Abstände von einem Stichprobenbaum zu seinen $k$ nächsten Nachbarn oder von einem Stichprobenpunkt zu den $k$ nächsten Bäumen gemessen. Dieses Auswahlschema führt zu einer konstanten Probebaumanzahl je Stichprobenpunkt mit je nach lokaler Bestandesdichte (Stammzahl je Flächeneinheit) variierender Probeflächengröße. Für konventionelle Probekreise gilt das Gegenteil, dort ist die Probefläche konstant und die Anzahl der erfaßten Bäume variiert.

Der Vorteil der Abstandsverfahren ist ihre Einfachheit in der Anwendung und der Effekt, daß sich der Arbeitsaufwand je Stichprobenpunkt bei steigender (lokaler) Bestandesdichte nicht erhöht, sondern aufgrund der geringeren (ideellen) Probefläche und der damit verbundenen kürzeren Laufwege eher verringert (vgl. PRODAN 1968; Ko et al. 1969; Lessard et al. 1994; SHEIL et al. 2003). KLEINN und VILČKO (2006b) weisen zudem daraufhin, daß aufgrund dieses Effektes die Arbeitszeit je Stichprobenpunkt wesentlich weniger variiert als bei der Aufnahme von Probekreisen mit festem Radius und dadurch die Planung und Kostenkalkulation einer Inventur erleichtert wird.

Allerdings stellte sich schon früh heraus, daß bei allen Abstandsverfahren die Genauigkeit der Stammzahl- und Grundflächenschätzung vom räumlichen Verteilungsmuster der Bäume abhängt und für jeden der zahlreich entwickelten Schätzer realistische Baumverteilungen vorstellbar sind, bei denen ein mehr oder weniger deutlicher systematischer Fehler (Bias) auftritt (LOETSCH et al. 1973, S. 369). Dies gilt vor allem bei der Messung von BaumBaum-Abständen, da der zugehörige Stammzahlschätzer in Beständen mit geklumpter Baumverteilung kaum auf Bestandeslücken reagiert (STOFFELS 1955) ${ }^{1}$. Aber auch für die Verfahren, die - wie die hier untersuchte - auf der Messung von Punkt-Baum-Abständen beruhen, konnten bis jetzt keine Schätzer gefunden werden, die für alle Typen räumlicher Vertei-

\footnotetext{
${ }^{1}$ Da bei dieser Methode der vom Stichprobenpunkt aus gesehen nächste Baum als Bezugsbaum für die Abstandsmessungen genutzt wird, findet in Bestandeslücken immer eine Verlagerung der Abstandsmessungen an den Rand des nächsten Baumklumpens statt, wodurch der mittlere Baumabstand systematisch unterschätzt wird.
} 
lungsmuster unverzerrte Ergebnisse liefern (vgl. COX 1971, S. 42; PAYANDEH und EK 1986; PICARD et al. 2005).

Diese Arbeit beschäftigt sich mit der Optimierung der von PRODAN (1968) vorgestellten 6-Baum-Stichprobe (SEBAST), bei der die ideelle Probekreisfläche durch den Abstand vom Stichprobenpunkt zur Stammachse des sechstnächsten Baumes bestimmt wird. Dabei geht es insbesondere um die Quantifizierung des Zusammenhangs zwischen der Art der räumlichen Verteilung und dem Bias der Stammzahlschätzung mit Hilfe eines Aggregationsindexes, basierend auf einem Vorschlag von PAYANDEH und EK (1986). Auf der Grundlage des daraus abgeleiteten Modells ist eine weitgehende Eliminierung des Bias möglich. Desweiteren werden aufnahmetechnische Modifikationen vorgeschlagen, mit denen sowohl die Anwendung erleichtert als auch die statistische Effizienz erhöht wird.

\section{Stichprobeneffizienz}

Wenn man die Wahl zwischen verschiedenen Inventurmethoden hat, wird man - bei rationaler Vorgehensweise - diejenige auswählen, die unter den zu erwartenden Umständen die effizienteste ist. Dabei beinhaltet der Begriff „Effizienz“ nach STAGE und RENNIE (1994) zwei Komponenten, nämlich die statistische Effizienz und die Einfachheit der Anwendung.

Die statistische Effizienz kann mit Hilfe des Konzeptes der Einschlußfläche analysiert werden $^{2}$, das hier am Beispiel einer Stichprobe mit Probekreisen konstanter Größe erläutert werden soll: Um in einem solchen Fall festzustellen, ob an einem Stichprobenpunkt ein bestimmter Baum erfaßt wird oder nicht, wird normalerweise der Abstand vom Probekreismittelpunkt zur Stammachse des Baumes gemessen und der Baum wird gezählt, wenn dieser Abstand kleiner ist als der Probekreisradius. Umgekehrt kann man aber auch vom Baum in Richtung Stichprobenpunkt messen. Dann wird gedanklich ein Kreis mit dem Radius des Probekreises um den Baum gelegt und der Baum wird gemessen, wenn der Kreis den Stichprobenpunkt einschließt. Folglich ist diejenige Fläche, in die ein Stichprobenpunkt fallen muß, damit ein bestimmter Baum erfaßt wird (in diesem Fall also die konstante Probekreisfläche), die Einschlußfläche dieses Baumes. Die Wahrscheinlichkeit, daß dieser Baum von der Stichprobe erfaßt wird, entspricht dann dem Quotient aus Einschlußfläche und Gesamtfläche des Bestandes. Da die Einschlußfläche in diesem Fall konstant ist, hätten hier alle Bäume die gleiche Auswahlwahrscheinlichkeit.

\footnotetext{
${ }^{2}$ GROSENBAUGH (1955) beschrieb diesen von HUSCH et al. (1993) später auch „tree-centred concept“ genannten Ansatz als erster und bezeichnete die Einschlußfläche als „K-circles“; in der späteren Literatur synonym verwendete Begriffe sind „area of influence“ (STAGE und RENNIE 1994) und „inclusion zone“ (KLEINN und VILČKO 2006b).
} 
Anders bei der Winkelzählprobe nach BITTERLICH (1948), bei der die Einschlußfläche einem Kreis entspricht, dessen Größe positiv proportional zur Baumgrundfläche ist. Für den Fall der $k$-Baum-Stichprobe (als verallgemeinerte Form der 6-Baum-Stichprobe) haben KLEINN und VILČKO (2006b) gezeigt, daß die Einschlußfläche durch das k-order Voronoi Polygon (OKABE et al. 1999) gebildet wird, dessen Form und Größe allein durch die relative räumliche Anordnung der Nachbarbäume bestimmt wird (wobei die Anzahl der Nachbarn, die einen Einfluß ausüben, um einiges größer sein kann als $k$ ). Stark vereinfachend läßt sich sagen: Je höher die lokale Stammzahl je Flächeneinheit, desto kleiner die Einschlußfläche und damit die Auswahlwahrscheinlichkeit und umgekehrt (Abb. 1, vgl. a. HIRNER 1978, S. 4 f.). Im Gegensatz zur Winkelzählprobe ist die Größe der Einschlußfläche bei der 6-BaumStichprobe also mit keinem Baumattribut korreliert, zumindest nicht direkt.

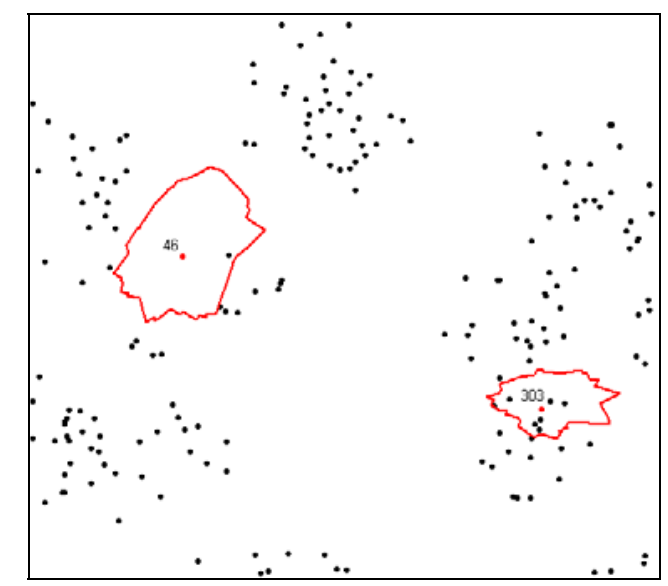

Abb. 1. Die Einschlußfläche zweier Bäume bei Anwendung der 6-Baum-Stichprobe innerhalb eines Bestandes mit geklumpter räumlicher Verteilung. Sie wird durch alle (Stichproben-)Punkte gebildet, für die der betreffende Baum der erst-, zweit-, ..., sechstnächste Baum ist. Demnach hat Baum 46 eine rund 2,5 mal höhere Auswahlwahrscheinlichkeit als Baum 303. Die Grafik wurde mit Hilfe des von VILČKO (2006) entwickelten Programms InventoryMap erstellt.

The inclusion zone of two trees within a stand with a clumped spatial pattern, when the six-tree-sample is applied. These zones are defined by all (sample) points, for which the relevant tree is the first, second, ..., sixth nearest one. According to this scheme, tree no. 46 has a 2.5 times greater probability of being selected in a sample than tree no. 303. The graph was generated using the software InventoryMap, developed by VILCKO (2006).

Basierend auf diesem Konzept kann die Effizienz einer Stichprobeninventur durch eine dem Inventurzweck angepaßte Definition der Einschlußfläche optimiert werden. STAGE und RENNIE (1994) führen hierzu aus, daß die statistische Effizienz vom kombinierten Effekt zweier Variationsquellen abhängt. Eine davon ist die Baum-zu-Baum-Varianz des Verhältnisses des interessierenden Baumattributes zur Einschlußfläche. So wird z. B. die Stammzahl pro ha am effizientesten mit Probekreisen konstanter Größe geschätzt, da das Verhältnis von Häufigkeit (1 für einen einzelnen Baum) und Einschlußfläche (konstant in diesem Fall) für alle Bäume gleich und somit dessen Varianz gleich 0 ist. Die Einschlußfläche und damit 
die Auswahlwahrscheinlichkeit eines bestimmten Baumtyps ist bei diesem Design ausschließlich abhängig von und proportional zu seiner Häufigkeit (PPF-Stichprobe ${ }^{3}$ ). Die Winkelzählprobe hingegen liefert ein konstantes Verhältnis von Baumgrundfläche und Einschlußfläche, hier ist die Auswahlwahrscheinlichkeit also proportional zu einem Größenmerkmal (PPSStichprobe ${ }^{4}$ ). Dementsprechend hat sich die Winkelzählprobe als das effektivste Inventurverfahren zur Schätzung der Grundfläche pro ha erwiesen (z. B. LOETSCH et al. 1973, S. 364).

Bei der 6-Baum-Stichprobe ist die Einschlußfläche weder zur Häufigkeit, noch zur Baumgrundfläche proportional. Da sie allein durch die räumliche Anordnung und somit durch die Abstände zu und zwischen den Nachbarbäumen bestimmt wird, steht sie lediglich in einem mehr oder weniger straffen Zusammenhang zur lokalen Stammzahl pro ha und damit zur lokalen mittleren Standfläche. Da in ungestörten, einschichtigen Beständen Standfläche und Baumgrundfläche positiv korreliert sein können (vgl. HIRNER, 1978, S. 25 ff.; UTSCHIG, 2002) und die Größe der Einschlußfläche positiv korreliert ist mit der lokalen mittleren Standfläche, werden unter solchen Verhältnissen Bäume mit größeren Grundflächen im Durchschnitt auch größere Einschlußflächen haben, so daß die Baum-zu-Baum-Varianz des Verhältnisses von Baumgrundfläche und Einschlußfläche geringer sein wird als bei der Anwendung von Probekreisen mit festem Radius. In diesen (wohl nicht allzu häufigen) Fällen ist also in Bezug auf die Schätzung der Grundfläche pro ha - bei gleicher durchschnittlicher Größe der Stichprobeneinheiten ${ }^{5}$ - eine höhere statistische Effizienz der 6-Baum-Stichprobe zu erwarten.

Bei nicht oder nur schwach vorhandener Korrelation von Baumgrundfläche und Einschlußfläche hingegen wird die statistische Effizienz der 6-Baum-Stichprobe im Vergleich zur Stichprobe mit festen Probekreisen umso geringer sein, je stärker geklumpt die Baumverteilung ist. Denn dann steigt die Varianz der Einschlußfläche (die bei Probekreisen konstanter Größe gleich 0 ist) und damit auch die Varianz des Verhältnisses von Baumgrundfläche und Einschlußfläche.

Hinsichtlich der Schätzung der Stammzahl pro ha gilt letzteres sinngemäß; hier ist allein das Verteilungsmuster und damit die Varianz der Einschlußfläche ausschlaggebend, da der Zähler des betrachteten Verhältnisses, hier also die Häufigkeit eines (einzelnen) Baumes, immer 1 ist. Die Effizienz der Schätzung mit der 6-Baum-Stichprobe wird sich also umso mehr der Effizienz fester Probekreise annähern, je regelmäßiger die Bäume verteilt sind. Im Vergleich mit der Winkelzählprobe ist ebenfalls allein entscheidend, welches Verfahren eine höhere Varianz der Einschlußflächen erzeugt. Da die Einschlußfläche bei der Winkelzählprobe durch die Baumgrundfläche und bei der 6-Baum-Stichprobe, wie gesagt, durch das räumliche Verteilungsmuster bestimmt wird, wird letztere - in Relation zur Winkelzählprobe - umso

\footnotetext{
${ }^{3}$ PPF: Probability proportional to frequency

4 PPS: Probability proportional to size

${ }^{5}$ D. h. bei einem Radius der festen Probekreise, der so gewählt wurde, daß im Durchschnitt ebenfalls 6 Bäume je Stichprobenpunkt erfaßt werden. Diese Annahme gilt bei allen hier angestellten Vergleichen.
} 
effizienter sein, je regelmäßiger die räumliche Verteilung und/oder je stärker die Spreitung der Baumdurchmesser ist. Diese theoretischen Überlegungen, die übrigens den bei bestimmten Verteilungsmustern zu erwartenden Bias bewußt unberücksichtigt lassen, werden durch die Ergebnisse einer von KLEINN und VILČKO (2006a) durchgeführten Studie, in der u. a. die Schätzgüte der genannten Verfahren in Bezug auf die Grundfläche und Stammzahl pro ha untersucht wird, weitgehend bestätigt.

Der zweite Faktor, der die statistische Effizienz beeinflußt, ist nach STAGE und RENNIE (1994) die Punkt-zu-Punkt-Varianz der Summe der oben genannten Verhältnis-Werte, die wiederum von der durchschnittlichen Größe der Einschlußflächen abhängt, im Fall der $k$ Baum-Stichprobe also vom gewählten $k$. Die Anzahl Bäume, die pro Stichprobenpunkt erfaßt werden, ist allerdings auch eng verknüpft mit der Einfachheit der Anwendung. Zwar sinkt der Variationskoeffizient bei gegebenem Stichprobenumfang mit steigender Größe der Einschlußfläche (d. h. mit steigender Anzahl der je Stichprobenpunkt erfaßten Bäume), gleichzeitig aber wächst die Zahl der Kontrollmessungen, die notwendig sind, um den $k$-ten Baum korrekt zu bestimmen, überproportional. Dies gilt insbesondere für locker bestockte oder lückige Bestände und verhindert die einfache und schnelle Anwendung der $k$-Baum-Stichprobe, den wesentlichen Vorteil des Verfahrens, welcher die im Vergleich mit anderen Inventurmethoden geringere statistische Effizienz ausgleichen kann (vgl. Ko et al. 1969; LESSARD et al. 1994). SCHÖPFER (1969a, b) untersuchte den Effekt unterschiedlicher $k$ auf den Stichprobenfehler und stellte fest, daß vor dem genannten Hintergrund die hyperbolische Abnahme des Fehlers nicht rechtfertigt, mehr als 6 Bäume je Stichprobenpunkt aufzunehmen; eine Aussage, die durch die Ergebnisse der Studie von KLEINN und VILČKO (2006a) ebenfalls bestätigt wird.

Die hier angestellten Überlegungen bilden - abgesehen von der grundsätzlichen Bedeutung für die Einordnung des Verfahrens - die Grundlage für deren später vorzustellende aufnahmetechnische Optimierung, die vor allem die Einfachheit und Schnelligkeit bei der Anwendung erhöhen und damit den, wie gezeigt wurde, entscheidenden Vorteil des Verfahrens weiter ausbauen soll.

\section{Schätzung der Stammzahl pro Hektar}

Ausgangspunkt der Überlegungen zur Korrektur des Bias der Stammzahlschätzung ist die Tatsache, daß für Bestände, deren räumliches Verteilungsmuster einem homogenen PoissonProzeß (RIPLEY 1981, S. 13 f.) entspricht, d. h. deren Bäume vollständig zufällig auf der Fläche verteilt sind (Poisson-Wald), ein auch theoretisch unverzerrter Schätzer existiert (s. Abschnitt 3.1). JONSSON et al. (1992) konnten zeigen, daß bei Anwendung dieses Schätzers eine Klumpung der räumlichen Baumverteilung einen positiven Bias verursacht, während ein negativer Bias in Populationen mit mehr oder weniger regelmäßigem Verteilungsmuster auftritt. Dabei sinkt der Betrag des Bias mit zunehmender Größe der Stichprobeneinheit, d. h. je mehr 
Bäume pro Stichprobenpunkt erfaßt werden, desto geringer ist die Verzerrung (SLOBODA 1976, S. 34) ${ }^{6}$. Diese Sensitivität gegenüber der Art der räumlichen Verteilung (vgl. z. B. CoX 1971; SMALTSCHINSKI 1981) kann geradezu als ein Charakteristikum der Abstandsverfahren bezeichnet werden, weswegen diese in Untersuchungen zu ökologischen Fragestellungen oft verwendet wurden (Diggle et al. 1976; PIELOU 1977; BYTH und RIPLEY 1980). PAYANDEH und EK (1986) schlugen daher vor, einen Index zur Beschreibung des Baumverteilungsmusters in den Stammzahlschätzer zu integrieren.

\subsection{Der Schätzer im Fall der Zufallsverteilung}

Bei der 6-Baum-Stichprobe ist die Stichprobeneinheit definiert durch die vom Stichprobenpunkt $i$ aus gesehen sechs nächsten Bäume, mit $i=1 . . n$. An jedem der systematisch (AKÇA 2001, S. 41 f.) über die Bestandesfläche verteilten $n$ Stichprobenpunkte werden die Art, der Stammdurchmesser in 1,3 m Höhe (BHD) und gegebenenfalls weitere Attribute der Probebäume erhoben. Der Abstand vom Stichprobenpunkt zur Stammachse des sechstnächsten Baumes liefert den Radius $r_{i}$ des ideellen Probekreises (s. Abb. 2).

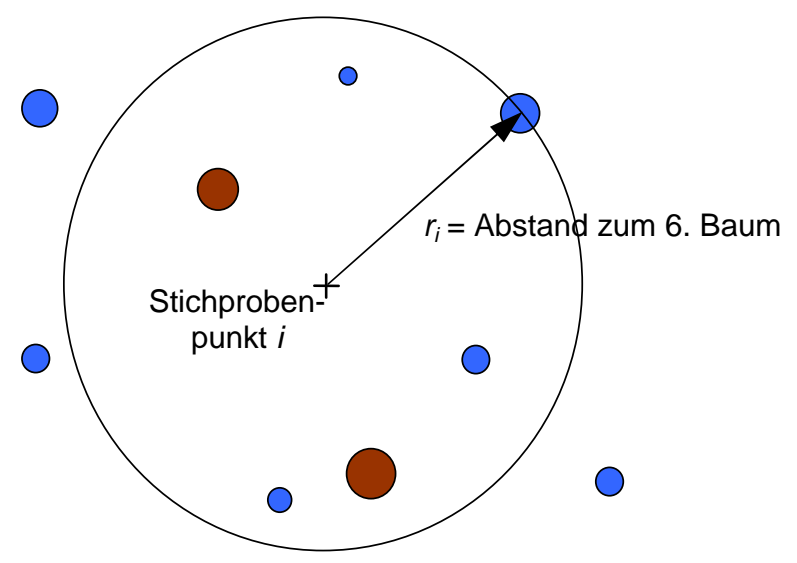

Abb. 2. Schema eines Probekreises der 6-Baum-Stichprobe. Der Probekreisradius entspricht dem Abstand vom Stichprobenpunkt zur Stammachse des sechstnächsten Baumes, die Probekreisfläche ist folglich $a_{i}=\pi \cdot r_{i}^{2}$.

Schematic diagram of a six-tree-sample plot. The radius of the sample plot is determined by the distance from the plot centre to the stem axis of the sixth nearest tree; hence, the plot size equals $a_{i}=\pi \cdot r_{i}^{2}$.

\footnotetext{
${ }^{6}$ Da bei der 6-Baum-Stichprobe die Größe der Stichprobeneinheit fixiert ist, wurde in dieser Arbeit nur der Einfluß des Verteilungsmusters untersucht.
} 
Nach JONSSON et al. (1992) ist dann im Fall eines Poisson-Waldes

$$
\hat{N}_{h a}=\frac{10000}{\pi \cdot n} \cdot \sum_{i=1}^{n} \frac{5}{r_{i}^{2}}
$$

ein erwartungstreuer Schätzer für die Stammzahl pro ha7.

\subsection{Das punktbezogene Winkelmaß $W_{P}$}

Da Schätzer (1) in Beständen mit einem von der Zufallsverteilung abweichenden Verteilungsmuster nicht erwartungstreu ist, wird ein numerischer Index benötigt, mit dem sich die räumliche Verteilung der Einzelbäume eines Bestandes quantifizieren läßt ${ }^{8}$. Für den hier verfolgten Zweck muß ein solcher Aggregationsindex einerseits möglichst sensitiv sein, sich andererseits aber auch schnell und einfach erheben lassen. Das punktbezogene Winkelmaß $W_{P}$ (STAUPENDAHL und ZUCCHINI 2006), das auf dem von GADOW et al. (1998) vorgestellten Ansatz beruht, Winkel zwischen benachbarten Bäumen nach ihrer Größe zu klassifizieren, erfüllt diese Anforderungen in besonderem Maße. Ursprünglich basiert es auf den vom Stichprobenpunkt aus gesehen drei nächsten Bäumen, wurde hier aber auf die ja bereits im Zuge der 6Baum-Stichprobe zu ermittelnden sechs nächsten Bäume erweitert. Dadurch entfällt die Notwendigkeit, innerhalb der primären Stichprobeneinheit eine Untereinheit zu identifizieren, was in Zweifelsfällen zusätzliche Abstandsmessungen und damit einen höheren Arbeitsaufwand erfordern würde. Das grundsätzliche Vorgehen aber bleibt dasselbe:

Für jeden Baum $j$ der Stichprobeneinheit $i$ wird der vom Stichprobenpunkt ausgehende Winkel $\alpha_{i j}$ zum jeweiligen Nachbarbaum bestimmt (mit $j=1 . .6$ ), wobei als Nachbar der in einer vorher festgelegten Drehrichtung als nächstes folgende Baum betrachtet wird. Einen Sonderfall stellt die Situation dar, in der der Winkel zwischen zwei benachbarten Bäumen mehr als $180^{\circ}$ beträgt. Zwischen diesen Bäumen wird dann in umgekehrter Richtung gemessen, $\alpha_{i j}$ ist also immer $\leq 180^{\circ}$ (vgl. Abb. 3). Jeder dieser Winkel wird mit dem sogenannten kritischen Winkel $\alpha_{c}=35^{\circ}$ verglichen, über den eine Klassifizierung in kleine (Indikator für Klumpung) und große Winkel (Indikator für Regelmäßigkeit) erfolgt ${ }^{9}$. Das punktbezogene

\footnotetext{
${ }^{7}$ Die allgemeine Form dieses Schätzers für jede beliebige Anzahl Bäume je Stichprobeneinheit, bezeichnet mit $k$, lautet: $\hat{N}_{h a}=\frac{10000}{\pi \cdot n} \cdot \frac{k-1}{k} \cdot \sum_{i=1}^{n} \frac{k}{r_{i}^{2}}$ (vgl. a. LESSARD et al. 2002).

8 Einen guten Überblick über die existierenden Aggregationsindizes vermitteln die Arbeiten von GLEICHMAR und Gerold (1998), UptON und Fingleton $(1985,1989)$ und SMALTSCHINSKI $(1998)$.

${ }^{9}$ STAUPENDAHL und ZUCCHINI (2006) entwickelten einen auf dem Winkelmaß beruhenden Test auf vollständig zufällige Verteilung, dessen Stärke - wie gezeigt werden konnte - vom kritischen Winkel $\alpha_{c}$ abhängt. Demzufolge sollte der kritische Winkel so gewählt werden, daß er die Teststärke für eine gegebene Alternativhypothese maximiert. Bei Berücksichtigung von sechs Bäumen je Stichprobenpunkt und für die in der Inventurpraxis rele(s. auch nächste Seite)
} 
Winkelmaß ist demnach definiert als der Anteil der Winkel $\alpha_{i j}$, die kleiner sind als der kritische Winkel $\alpha_{c}$ :

$$
W_{P, i}=\frac{1}{6} \cdot \sum_{j=1}^{6} z_{i j}, \text { mit } z_{i j}=\left\{\begin{array}{ll}
1 & \text { falls } \alpha_{i j}<\alpha_{c} \\
\odot & \text { sonst }
\end{array}, \alpha_{c}=35^{\circ} \text { und } 0 \leq W_{P, i} \leq 1\right.
$$

Aus (2) folgt, daß $W_{P, i}$ nur 7 verschiedene Werte annehmen kann, nämlich 0, 0,17, 0,33, 0,5, $0,67,0,83$ und $1 ; W_{P, i}=0$ deutet auf eine stark ausgeprägte Regelmäßigkeit hin, während bei $W_{P, i}=1$ eine deutliche Klumpung zu erwarten ist (Abb. 3 zeigt zwei Beispiele).
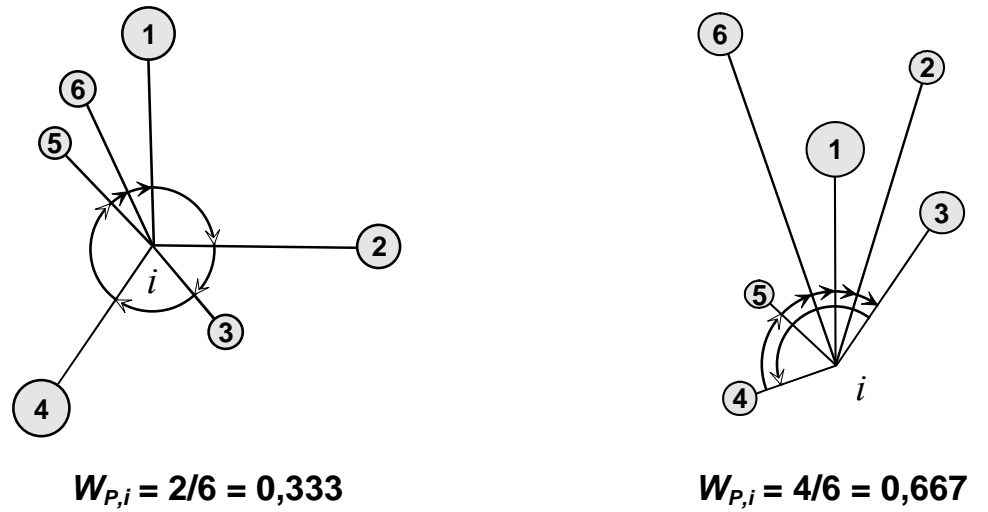

Abb. 3. Zwei mögliche Werte des punktbezogenen Winkelmaßes $W_{P}$ an einem Punkt der 6-Baum-Stichprobe bei einem kritischen Winkel $\alpha_{c}=35^{\circ}$. Die Bäume sind hier nach ihrem Azimut (nicht nach ihrem Abstand zum Probepunkt!) aufsteigend numeriert. Der Nachbar eines Baumes ist also durch die nächst größere Baumnummer gekennzeichnet. Ist die Pfeilspitze des zugehörigen Winkelbogens schwarz, ist $\alpha_{i j}$ kleiner als $\alpha_{c}$, andernfalls größer. Die Abbildung rechts macht deutlich, daß zwischen zwei benachbarten Bäumen immer der kleinere der beiden möglichen Winkel gemessen wird!

Two possible values of the point-based Winkelmass $W_{P}$ at a six-tree-sample point, with critical angle $\alpha_{c}=35^{\circ}$. The trees are numbered in ascending order with reference to their azimuth (not to their distance to the sample point!). Consequently, the neighbour of a tree is denoted by the next higher tree number. If the arrowhead of the associated angle arc is black, $\alpha_{i j}$ is smaller then $\alpha_{c}$, and greater otherwise. The right figure illustrates that between two neighbouring trees it is always the smaller one of the two possible angles that is measured.

Ausgehend von einer systematischen Stichprobe des Umfangs $n$ (wie oben beschrieben), ist

$$
\hat{W}_{P}=\frac{1}{n} \cdot \sum_{i=1}^{n} W_{P, i}
$$

ein erwartungstreuer Schätzer für den $W_{P}$-Wert des Bestandes. Wie bei allen Aggregationsindizes besteht auch beim Winkelmaß am Bestandesrand generell eine höhere Tendenz zur Klumpung, da jenseits dieser Grenze keine Bäume ausgewählt werden sollen oder können

vante, unspezifische Alternative „Die Verteilung ist entweder regelmäßiger oder geklumpter als bei Zufallsverteilung" ist dies bei $\alpha_{c}=35^{\circ}$ der Fall. 
(Randeffekt). Liegt der Stichprobenpunkt z. B. direkt neben einer gerade verlaufenden Bestandesgrenze, liegt das theoretische Minimum des $W_{P, i}$-Wertes selbst bei absolut regelmäßiger Verteilung der Randbäume bei 0,17, da der Suchwinkel für die sechs nächsten Bäume auf $180^{\circ}$ beschränkt ist. Deshalb sollten bei der Berechnung des Winkelmaßes gemäß Schätzer (3) nur diejenigen Probekreise berücksichtigt werden, die den Bestandesrand nicht schneiden. Dadurch ist jegliche Beeinflussung des $W_{P}$-Wertes durch Randeffekte ausgeschlossen.

Da für die Bestimmung des Winkelmaßes kein exakter Winkel benötigt wird, sondern nur geprüft werden muß, ob ein Winkel kleiner oder größer als ein bestimmter Grenzwert ist, läßt sich die Bestimmung des $W_{P, i}$-Wertes deutlich beschleunigen, wenn man das am Institut für Waldinventur und Waldwachstum der Universität Göttingen entwickelte „Winkelvisier“ verwendet: Dieses einfache Meßinstrument besteht aus zwei auf einem Stativ strahlenförmig befestigten, schmalen Schienen, auf denen eine Visierlinie angebracht ist und deren Winkel zueinander auf den kritischen Winkel eingestellt wird. Das Stativ wird am Stichprobenpunkt positioniert und mit einer der beiden Schienen wird die Stammachse des $j$-ten Baums anvisiert. Blickt man nun über die andere Schiene, läßt sich schnell und zuverlässig bestimmen, ob der Nachbarbaum inner- oder außerhalb des kritischen Winkels liegt. Mit Hilfe dieses Instruments läßt sich das Winkelmaß i.d.R. in weniger als 1 min. bestimmen.

\subsection{Stichprobensimulation}

Zur Bestimmung der Größenordnungen von Stichprobenfehler und Bias eines Inventurverfahrens ist die Stichprobensimulation auf der Basis von vollaufgenommenen Beständen ein probates Mittel, das heute dank leistungsfähiger Computer umfangreiche Tests erlaubt. Hierbei können bei gegebenem Stichprobenumfang durch wiederholte Probennahme mit jeweils unterschiedlicher Anordnung der Stichprobenpunkte alle möglichen Konstellationen berücksichtigt werden, was in der praktischen Durchführung einen enormen Arbeitsaufwand und damit sehr hohe Kosten verursachen würde.

Da für die hier interessierende Fragestellung Baumverteilungsmuster von extremer Regelmäßigkeit bis zu extremer Klumpung untersucht werden sollten, war es naheliegend, Bestände mit Hilfe von Punktprozessen (STOYAn und StOyAn 1992) künstlich zu erzeugen. Dabei war die Anwendung von nicht markierten Punktprozessen ausreichend, da hier allein die Verteilung der Baumpositionen relevant war, so daß die anderen Baumparameter (Baumart und BHD) konstant gehalten werden konnten.

Alle generierten Bestände haben eine Fläche $A=100 \mathrm{~m}$ x $100 \mathrm{~m}=1$ ha. Die Vorgabe bei der Generierung war die Erzeugung von 1000 Bäumen, die aber in Abhängigkeit von Zufallseffekten und vom gewählten Punktprozeß i.d.R. nicht exakt realisiert wurde. Bei allen Punktprozessen wurden die zugehörigen Parameter (in der folgenden Auflistung fett-kursiv gedruckt) so variiert, daß das gesamte Spektrum von regelmäßigen bzw. geklumpten Vertei- 
lungsmustern möglichst gleichmäßig abgedeckt wurde. Bei der Generierung der Bestände mit regelmäßiger Baumverteilung kamen vier verschiedene Punktprozesse zur Anwendung, wobei jeweils 10 Bestände erzeugt wurden:

- Randomly Displaced Lattice (RDL): Bei diesem Punktprozeß werden die Bäume in einem Rechteckgitter mit der Gitterzellenfläche $A_{g}=\boldsymbol{a} \times b\left(\right.$ hier $10 \mathrm{~m}^{2}$ ) und zufälligem Ausrichtungswinkel $\gamma$ über die Fläche verteilt, wobei die Gitternetzpunkte um einen zufälligen Vektor verschoben werden. Dieser Vektor folgt einer bivariaten Normalverteilung mit der Korrelation $\rho=0$, den Mittelwerten $\mu_{x}=\mu_{y}=0$ und den Standardabweichungen $\sigma_{x}=\sigma_{y}=\boldsymbol{\sigma} . \boldsymbol{a}$ wurde über eine gleichverteilte Zufallszahl bestimmt, $b$ ergab sich dann aus der Division von $A_{g}$ durch $\boldsymbol{a}$ (vgl. StOyAn und StOyan 1992, S. 307 ff.).

- Simple Sequential Inhibition (SSI): Der SSI-Prozeß erzeugt die Baumpositionen nacheinander, und zwar mit gleichverteilten und von bereits bestehenden Bäumen unabhängigen Koordinaten. Sollte ein neuer Baum jedoch weniger als $\boldsymbol{r}_{\boldsymbol{i}}$ Meter von einem schon existierenden entfernt sein, wird er verworfen und es wird solange ein weiterer Baum erzeugt, bis die vorgegebene Stammzahl erreicht ist. Um Endlosschleifen auszuschließen, wurde der Prozeß allerdings abgebrochen, wenn nach 10.000 Versuchen kein weiterer „freier Platz" gefunden werden konnte.

- Strauss Process (STR): Der Strauss-Prozeß verwendet wie der SSI-Prozeß einen „radius of inhibition" $r_{i}$ und den Parameter $\boldsymbol{c}$, mit $0 \leq \boldsymbol{c} \leq 1$. Ist $\boldsymbol{c}=1$, entspricht das Ergebnis einem homogenen Poisson-Prozeß, während $\boldsymbol{c}=0$ ein Verteilungsmuster erzeugt, in dem jeder Baum mindestens $r_{i}$ Längeneinheiten (hier 2,5 m) von seinem nächsten Nachbarn entfernt ist. Mit $c$ wird also der Grad der durch $r_{i}$ definierten, maximal möglichen „Abstoßung“ gesteuert.

- Matérn Hard Core, Typ II (MHC): Der Algorithmus des MHC-Prozesses erzeugt zunächst ein Muster von „Punktvorschlägen“, das einem gleichverteilten Poisson-Prozeß mit der Intensität $\lambda$ (hier 0.1) entspricht. Dabei erhält jeder Punkt eine „Ankunftszeit“, d. h. eine unabhängige, in $[0,1]$ gleichverteilte Zufallszahl. Anschließend werden alle „Punktvorschläge" gelöscht, die sich innerhalb einer Distanz von $\boldsymbol{r}_{\boldsymbol{i}}$ Metern zu einem anderen „Punktvorschlag“ mit einer früheren „Ankunftszeit“ befinden. Die übrigen „Punktvorschläge“" werden beibehalten. Daraus ergibt sich, daß die Anzahl der erzeugten Bäume bei gleicher Intensität $\lambda$ mit größer werdendem $\boldsymbol{r}_{\boldsymbol{i}}$ immer kleiner wird.

Für die Erzeugung der geklumpten Verteilungsmuster wurden drei verschiedene Punktprozesse genutzt, die alle Spezialfälle des Neyman-Scott-Prozesses darstellen. Hierbei werden in einem ersten Schritt „Elternpunkte“ erzeugt, deren Verteilung einem homogenen PoissonProzeß mit der Intensität $\lambda$ folgt. Jeder „Elternpunkt“ wird dann durch einen Klumpen mit $n_{c}$ Bäumen ersetzt, wobei $n_{c}$ eine Poisson $(\mu)$-verteilte Zufallszahl ist. Damit bei variierender 
Klumpengröße im Gesamtbestand konstant 1000 Bäume erzeugt wurden, wurde die Intensität des Poisson-Prozesses in Abhängigkeit von $\boldsymbol{\mu}$ gewählt, mit $\lambda=(1000 / \boldsymbol{\mu}) / A$. Die Positionierung der Bäume innerhalb der Klumpen ist abhängig vom gewählten Punktprozeß:

- Modified Thomas (MTH $\rightarrow 20$ Bestände): Hier werden die Baumpositionen dadurch erzeugt, daß zur Position des „Elternpunktes“ ein Vektor addiert wird, dessen zufällige Werte einer bivariaten Normalverteilung mit der Korrelation $\rho=0$, den Mittelwerten $\mu_{x}=\mu_{y}=0$ und den Standardabweichungen $\sigma_{x}=\sigma_{y}=\sigma$ folgen.

- Matérn Cluster (MCL $\rightarrow 20$ Bestände): Der Matérn Cluster-Prozeß platziert die Bäume gleichverteilt und unabhängig innerhalb einer um den Elternpunkt gedachten Kreisfläche mit dem Radius $r_{c}$.

- SSI Cluster (MIX $\rightarrow 27$ Bestände): Dieser Prozeß wurde durch den Autor selbst konstruiert und erzeugt die Baumpositionen ebenfalls innerhalb des Radius $\boldsymbol{r}_{\boldsymbol{c}}$ um den Elternpunkt, folgt dabei aber einem SSI-Prozeß (s. o.). Dessen ,,radius of inhibition“ $\boldsymbol{r}_{\boldsymbol{i}}$ wird hier in Abhängigkeit von dem Parameter $d$ und dem mittleren Baumabstand innerhalb eines Klumpens berechnet, mit $r_{i}=d \cdot \sqrt{\left(\pi \cdot r_{c}^{2}\right) / n_{c}}$ und $0 \leq d \leq 1$. Somit steuert $d$ den Grad der in einem Klumpen maximal möglichen „Abstoßung“.

Bis auf die RDL-Punktmuster, die mit Hilfe des Programms STG von STOYAN (1995) generiert wurden, wurden alle anderen mit den entsprechenden Funktionen der Pakete Spatial (VENABLES und RiPley 2002) und Spatstat (BADDELEY und TURNER 2006) des Statistikprogramms $R$ (R DEVELOPMENT CORE TEAM 2005) erzeugt.

In jedem dieser 107 Bestände wurde eine 6-Baum-Stichprobe mit 50 Stichprobenpunkten 1000 mal wiederholt. Die Probepunkte wurden mit Hilfe eines quadratischen Gitternetzes über die Bestandesfläche verteilt, wobei Startpunkt und Ausrichtungswinkel bei jeder Wiederholung zufällig gewählt wurden (systematische Stichprobe mit Zufallsstart). Eine systematische Unterschätzung der Stammzahl wird durch die Probekreise verursacht, die den Bestandesrand schneiden, da außerhalb der Bestandesfläche keine Bäume vorhanden sind bzw. erfaßt werden. Ein anerkanntes Verfahren zur Korrektur dieses Randfehlers ist die Spiegelungsmethode nach SCHMID-HAAS (1969b), die jedoch an Bestandesecken, wo der Probekreis von mehr als einer Bestandesgrenze geschnitten wird, nur unvollständig möglich ist (Pommerening 1997, S. 41). Daher wurde stattdessen für jeden am Rand liegenden Probekreis der Anteil der ideellen Probekreisfläche ermittelt, der innerhalb der Bestandesfläche liegt (vgl. BEERS 1966). Zur Hochrechnung der Stammzahl auf ha-Werte wurde dann nur diese Teilfläche $a_{i}^{i n}$ verwendet (s. Formel (1), in diesen Fällen ist also $r_{i}=\sqrt{a_{i}^{\text {in }} / \pi}$ ).

Für die praktische Durchführung der Simulation wurde unter Borland Delphi (Programmiersprache Pascal) der Stichprobensimulator PoiSSim entwickelt (STAUPENDAHL 2001). Dieses Programm simuliert die wiederholte Stichprobenziehung in einem Bestand und 
speichert nach jedem Simulationsdurchgang die geschätzte Stammzahl pro ha, das Winkelmaß und weitere Bestandes- und Stichprobenparameter, so daß am Ende der Simulation die Erwartungs- und Biaswerte berechnet werden können. Der Erwartungswert eines Parameterschätzers $\hat{\theta}$ ist der Mittelwert der Schätzergebnisse für alle möglichen Stichproben $r$, mit $r=$ $1 . . m$ und $m \rightarrow \infty$. Mit der hier gewählten Anzahl an Stichprobenwiederholungen $(m=1000)$ wird eine ausreichend genaue Schätzung erreicht. Der Bias ist dann die Differenz aus Erwartungswert und wahrem Wert: $\operatorname{Bias}(\hat{\theta})=E(\hat{\theta})-\theta$. Zur Analyse der resultierenden Daten wurde das Statistik-Programm $R$ (R DeVELOPMENT CoRE TEAm 2005) verwendet.

\subsection{Der Bias der Stammzahlschätzung}

Die Simulationsdaten zeigten, daß das punktbezogene Winkelmaß $W_{P}$ in einem sehr straffen, nichtlinearen Zusammenhang zum relativen Bias der Stammzahlschätzung steht (Abb. 4). Als gut angepaßtes, aber dennoch einfaches Modell zur Beschreibung dieses Zusammenhangs erwies sich die Gleichung

$$
\begin{aligned}
& \operatorname{rBias}\left(\hat{N}_{h a}\right)=c_{1}+c_{2} \cdot E\left(\hat{W}_{P}\right)^{c_{3}}, \\
& \operatorname{mit} \operatorname{rBias}\left(\hat{N}_{h a}\right)=\frac{E\left(\hat{N}_{h a}\right)-N_{h a}}{N_{h a}}=\frac{E\left(\hat{N}_{h a}\right)}{N_{h a}}-1 .
\end{aligned}
$$

Die mit Hilfe der nichtlinearen Regressionsrechnung ermittelten Koeffizienten sind in Tab. 1 wiedergegeben.

Tab. 1. Koeffizienten der Regressionsgleichung zur Schätzung des bei der 6-Baum-Stichprobe zu erwartenden relativen Bias der Stammzahl pro ha.

Coefficients of the regression equation, which is used to estimate the relative bias of the stem number per ha produced by the six-tree-sample.

\begin{tabular}{cccc}
\hline Koeffizient & Wert & Standardfehler & Signifikanzniveau \\
\hline$c_{1}$ & $-0,075402$ & 0,005827 & 0,001 \\
$c_{2}$ & 1,358629 & 0,035418 & 0,001 \\
$c_{3}$ & 3,109408 & 0,092690 & 0,001 \\
\hline
\end{tabular}

Jeder der Koeffizienten des Modells ist hochsignifikant und mit 97,7 \% wird ein hoher Anteil der Streuung der Biaswerte erklärt. Wichtig ist vor allem der geringe Standardfehler der Residuen: $95 \%$ liegen innerhalb eines Intervalls von $\pm 5,2 \%$ und damit in einer Größenordnung, die für praktische Zwecke kaum von Bedeutung ist. Dabei gilt es zu bedenken, daß die Residuenstreuung in den stark geklumpten Beständen am stärksten ist (s. Abb. 4) und hier Bestän- 
de mit einem Klumpungsgrad erzeugt wurden, der in der Natur nur in den seltensten Fällen zu erwarten ist.

$$
\text { Gemäß Gleichung (4) gilt }
$$

$$
\begin{aligned}
\frac{E\left(\hat{N}_{h a}\right)}{N_{h a}}-1 & =c_{1}+c_{2} \cdot E\left(\hat{W}_{P}\right)^{c_{3}} \\
\Leftrightarrow \quad N_{h a} & =\frac{E\left(\hat{N}_{h a}\right)}{1+c_{1}+c_{2} \cdot E\left(\hat{W}_{P}\right)^{c_{3}}},
\end{aligned}
$$

so daß der in Nicht-Poisson-Wäldern zu erwartende Bias des Schätzers (1) demnach auf ein vertretbares Maß reduziert werden kann, wenn die Schätzung aus (1) durch $1+\operatorname{rBias}\left(\hat{N}_{h a}\right)$ dividiert wird.

Der neue Schätzer der 6-Baum-Stichprobe für die Stammzahl pro ha lautet dann

$$
\hat{N}_{h a}=k f \cdot \frac{10000}{\pi \cdot n} \cdot \sum_{i=1}^{n} \frac{5}{r_{i}^{2}}, \text { mit } k f=\frac{1}{0,9246+1,3586 \cdot \hat{W}_{P}^{3,109}} .
$$

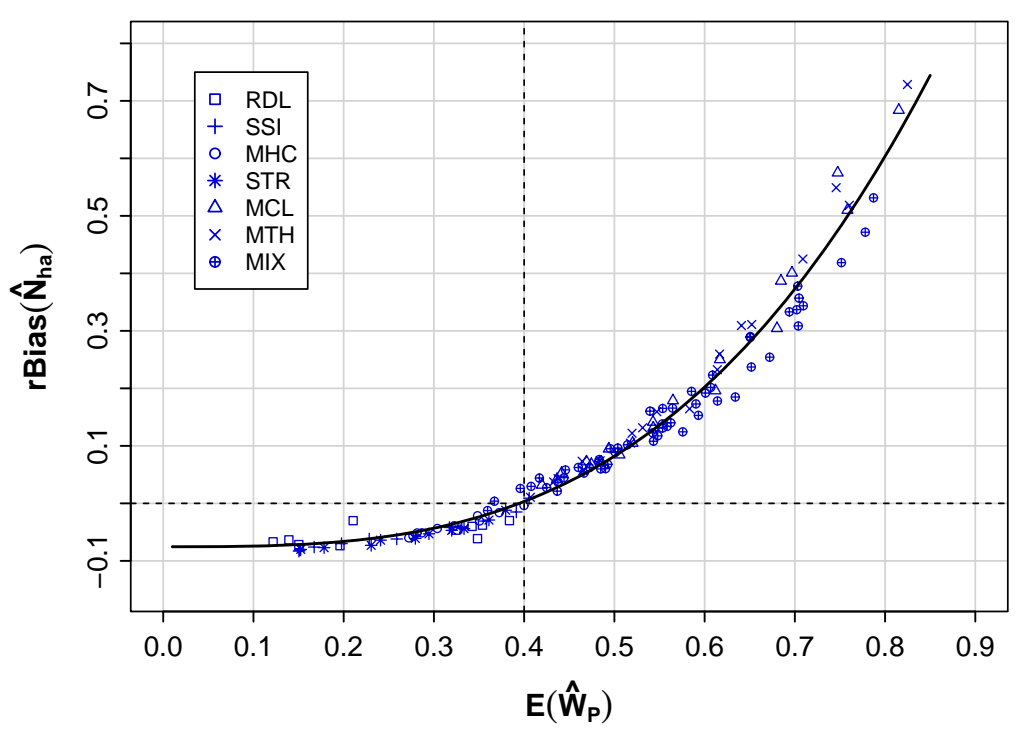

Abb. 4. Bias der Stammzahl pro ha bei der 6-Baum-Stichprobe (relativ zur wahren Stammzahl) in Abhängigkeit vom punktbezogenen Winkelmaß $W_{P}$. Die verschiedenen Punktsymbole bezeichnen die Punktprozesse, mit denen die zugehörigen Simulationsbestände erzeugt wurden, die Kurve beschreibt Regressionsgleichung (4), mit der die Punktewolke rechnerisch ausgeglichen wurde. Die vertikale punktierte Linie markiert den $W_{P}$-Wert eines „Poisson-Waldes“, bei dem im Mittel kein Bias auftritt.

The bias of the stem number per ha produced by the six-tree-sample (relative to the true stem number) against the point-based Winkelmass $W_{P}$. The different point symbols denote the point processes, which were used to generate the associated simulation stands, the curve represents regression equation (4), which was used to smooth the scatter plot. The vertical dotted line indicates the $W_{P}$-value of a ,poisson forest“, where on average no bias will occur.

Aus Formel (5) geht hervor, daß die Präzision der Schätzung des $W_{P}$-Wertes bei der Biaskorrektur eine wichtige Rolle spielt. Denn wenn $\hat{W}_{P}$ stark streut, wird der Korrekturfaktor im 
Mittel zwar korrekt sein, im Einzelfall den Bias aber über- oder unterschätzen, was je nach Vorzeichen des tatsächlichen Bias sogar zu einer Vergrößerung des Schätzfehlers führen kann (s. Abschnitt 6). Daneben fällt in Abb. 4 auf, daß der Zusammenhang zwischen Winkelmaß und Bias in den Beständen mit RDL-Punktmustern (gitternetzartiger Anordnung der Bäume) deutlich weniger straff ist. Bereits JONSSON et al. (1992) hatten beobachtet, daß der Stammzahlbias mit der Kantenlänge von gitternetzartigen Verteilungsmustern variiert und offensichtlich kann diese Varianz mit dem Winkelmaß nur ungenügend erklärt werden. Somit wäre die Biasschätzung in Beständen, die künstlich durch Reihenpflanzung begründet wurden und deren Reihenstruktur noch deutlich erkennbar ist, mit Unsicherheiten behaftet. Allerdings ist diese Einschränkung nicht sehr schwerwiegend, da der in solchen Beständen zu erwartende Bias nicht sehr hoch zu sein scheint.

Desweiteren wird aus der Streuung der Wertepaare im Bereich geklumpter Verteilungsmuster ersichtlich, daß bei gleichem $W_{P}$-Wert doch deutlich unterschiedliche Verteilungsmuster vorliegen können (s. Abb. 5), die dann auch unterschiedliche systematische Fehler erzeugen. Dies bedingt, daß mit dem hier entwickelten Modell z. B. für Bestände mit Verteilungsmustern, die dem „MIX“-Punktprozeß entsprechen, bei $W_{P}$-Werten $<0,5$ mit einer systematischen Unterschätzung, bei $W_{P}$-Werten darüber mit einer Überschätzung des Bias zu rechnen ist. $\mathrm{Ob}$ diese systematischen Fehler allerdings von praktischer Bedeutung sind, wird im Rahmen der späteren Validierung des Modells (Abschnitt 6) erläutert.

(a)

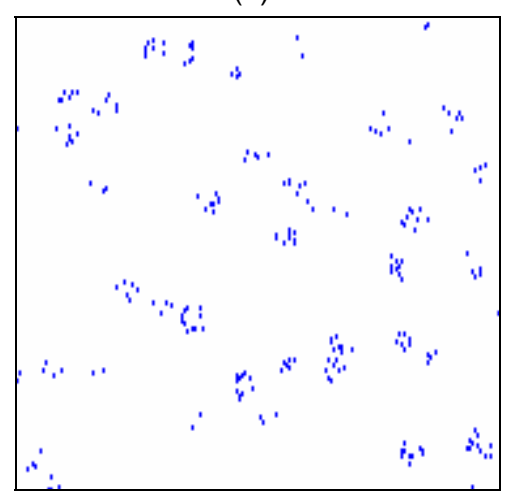

(b)

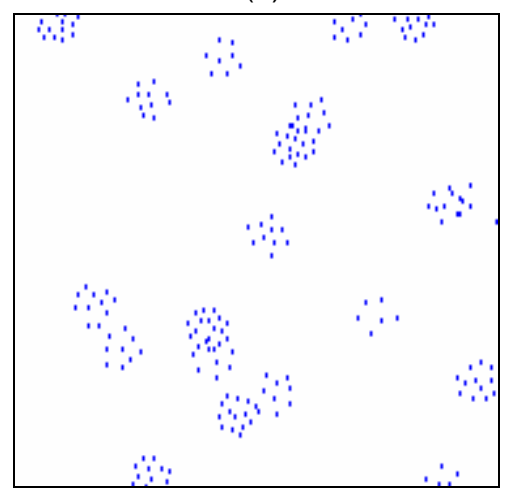

Abb. 5. Ausschnitte von zwei künstlich erzeugten, in der Stichprobensimulation verwendeten Beständen mit extrem geklumptem Verteilungsmuster. Bestand (a) ist eine Realisierung des Matérn Cluster Prozesses (MCL), während Bestand (b) mit Hilfe des SSI Cluster Prozesses (MIX) erzeugt wurde. Für beide Bestände beträgt der $W_{P}$-Wert ungefähr 0,75 .

Clippings from two artificially generated stands, used in the sample simulation, with an extreme clumped spatial pattern. Stand (a) is the outcome of a Matérn Cluster Process (MCL), whereas stand (b) was generated using the SSI Cluster Process (MIX). The $W_{P}$-value of both stands amounts to approximately 0.75 . 


\section{Schätzung der Grundfläche pro Hektar}

Die Schätzung der Grundfläche pro ha kann in die Komponenten Stammzahl pro ha und mittlere Baumgrundfläche zerlegt werden (vgl. a. JONSSON et al. 1992). Der Schätzer für die Grundfläche pro ha lautet dann

$$
\hat{G}_{h a}=k f \cdot \frac{10000}{\pi \cdot n} \cdot \sum_{i=1}^{n} \frac{5}{r_{i}^{2}} \cdot \bar{g}_{i}, \text { mit } \bar{g}_{i}=\frac{\pi}{40000 \cdot 6} \cdot \sum_{j=1}^{6} d_{i j}^{2}
$$

wobei $d_{i j}$ die Brusthöhendurchmesser der Bäume des $i$-ten Probekreises bezeichnet. SABOROWSKI und ŠMELKO (1998) konnten anhand einer stichprobentheoretischen Analyse und deren experimenteller Überprüfung zeigen, daß das arithmetische Mittel aus den auf Hektar hochgerechneten Probekreis-Grundflächen bei systematischen Stichproben mit variablen Probeflächengrößen ein erwartungstreuer Schätzer ist. Die dort in der theoretischen Beweisführung unterstellte vollständige Aufteilung des Inventurgebiets in sich nicht überlappende, quadratische Probeflächen stellt allerdings nur eine Approximation an den tatsächlichen Stichprobenraum der 6-Baum-Stichprobe dar, da es -wie die Autoren selber anführen - unendlich viele mögliche Stichprobeneinheiten gibt (vgl. a. KLEINN und VILČKO 2005). Weitere Probleme, die im Zusammenhang mit Schätzer (6) auftreten können, und Ansätze zu deren Lösung werden im folgenden Abschnitt behandelt.

\section{Aufnahmetechnische Modifikationen}

Da die Korrektur der verzerrten Stammzahlschätzung nur gesamthaft für den Mittelwert, nicht aber „probekreis-individuell“ erfolgt, kann bei der Schätzung der Grundfläche pro ha gemäß Gleichung (6) ein Bias dadurch entstehen, daß einzelne Probekreise ein zu hohes oder zu niedriges Gewicht erhalten. HIRNER (1978, S. 56 f.) beschrieb die Überlagerung der Standflächen mehrerer Einzelbäume in mehrschichtigen Beständen, wie sie z. B. in zweischichtigen Buchenbeständen mit stammzahlreichem Unterstand auftreten, als eine mögliche Ursache für solche Effekte. Wollte man einen solchen Bestand in Gänze mit der 6-BaumStichprobe erfassen, würden sehr viele unterständige Buchen aufgenommen, während die wertvollen Stämme des Hauptbestandes nur mit geringer Stammzahl in die Stichprobe eingingen. Neben dem dadurch evtl. erzeugten systematischen Fehler wären außerdem die Schätzungen für den Unterstand unnötig präzise und für den Hauptbestand zu unpräzise.

Auf der Ebene von permanenten Probekreisinventuren führten diese Überlegungen zur Einführung konzentrischer Probekreise (z. B. STARKE 1989). Als Konsequenz für die 6Baum-Stichprobe und nach dem Vorbild der im Baden-Württembergischen Betriebsinventurverfahren vorgesehenen, sogenannten doppelten 6-Baum-Stichprobe (BLUST et al. 2006) wird hier eine Stratifizierung nach Bestandesschichten vorgeschlagen, wobei nach Hauptbe- 
stand (HB) und Nebenbestand (NB) unterschieden wird, letzterer untergliedert in die Schichten Überhalt und Unterstand ${ }^{10}$. Die Auswahl der Probebäume der eigentlichen 6-BaumStichprobe erfolgt dann nur im Hauptbestand! An einem Stichprobenpunkt $i$ evtl. vorkommender Überhalt oder Unterstand wird dagegen durch eine okulare Schätzung der Stammzahl $n_{N B, i}$ und des Durchmessers des Grundflächenmittelstamms $d g_{N B, i}$ innerhalb eines festen Radius $r_{N B, i}$ (6 $\mathbf{~ m}$ im Unterstand und $\mathbf{1 5} \mathbf{~ m}$ im Überhalt) erfaßt. Dabei wird davon ausgegangen, daß an einem einzelnen Stichprobenpunkt zusätzlich zum Hauptbestand nur eine dieser beiden Schichten vorkommt. In Beständen ohne eindeutig abgrenzbare Schichten mit exponentiell abnehmender Durchmesserverteilung (z. B. in Plenterwäldern) sollte durch Festlegung eines Grenzdurchmessers ein Hauptbestand und Unterstand definiert werden, wobei dieser Grenzdurchmesser so zu wählen wäre, daß beide „Schichten“ möglichst gut getrennt werden, um die Zahl der Bäume zu minimieren, bei denen unklar ist, zu welchem Kollektiv sie gehören.

Der durch die okulare Schätzung zusätzlich entstehende Fehler, sowohl zufälliger als auch systematischer Art, dürfte nach einiger Übung in vertretbaren Grenzen zu halten sein, zumal die absolute und/oder relative Höhe der Grundfläche (als i.d.R. maßgebliche meßbare Variable einer Bestandesinventur) im Nebenbestand fast immer sehr gering ist ${ }^{11}$. Unter dem Aspekt höchstmöglicher Wirtschaftlichkeit ist das Ziel der Inventur hier vor allem die Erfassung des „Vorhandenseins“. Der Hauptbestand wird dagegen um so genauer geschätzt, so daß der Fehler der Stammzahl- und Grundflächenschätzung des Gesamtbestandes je nach Bestandesstruktur z. T. erheblich geringer sein dürfte als bei der 6-Baum-Stichprobe ohne Stratifizierung. Dieses Vorgehen entspricht auch den Überlegungen zur Stichprobeneffizienz in Abschnitt 2, da 1.) durch die Beschränkung der 6-Baum-Stichprobe auf den Hauptbestand die dort evtl. vorhandene Korrelation von Baumgrundfläche und Standfläche die statistische Effizienz steigern kann und 2.) die Stammzahl pro ha, als in den grundflächenarmen Bestandesschichten Überhalt (geringe Stammzahl) und Unterstand (geringe Stammdurchmesser) relevante Zielgröße, am effektivsten mit Probekreisen konstanter Größe geschätzt werden kann.

Ein weiterer wichtiger Aspekt ist die Begrenzung des Probekreisradius, der bei der 6Baum-Stichprobe normalerweise durch den Abstand des sechstnächsten Baumes zum Probekreismittelpunkt definiert ist. Durch diese Definition der Stichprobeneinheit ist die Durchführung der konventionellen 6-Baum-Stichprobe in schwach bestockten Bestandespartien und auf Blößen sehr erschwert. Die großen Meßdistanzen und die dadurch bedingt zeitaufwendige Ermittlung des 6. Baumes lassen in diesen Fällen den entscheidenden Vorteil des Verfahrens,

\footnotetext{
${ }^{10}$ Nach der niedersächsischen Forsteinrichtungsanweisung (NIEDERSÄCHSISCHE LANDESFORSTEN 1987) sind die Bestandesschichten folgendermaßen definiert: Hauptbestand: Bestandesschicht, auf der das wirtschaftliche Hauptgewicht liegt; Überhalt: Reste aus dem Vorbestand über dem Hauptbestand mit einem Bestockungsgrad $\leq 0,3$; Unterstand: Unter dem geschlossenen Hauptbestand wachsender Baum- und Strauchwuchs, [...].
} 
nämlich die einfache und rasche Merkmalserhebung, wieder hinfällig werden (HIRNER 1978, S. 70 f.). Deshalb werden in der modifizierten 6-Baum-Stichprobe nur diejenigen Bäume des Hauptbestandes berücksichtigt, die maximal 15 m vom Stichprobenpunkt entfernt sind (vgl. SHEIL et al. 2003) ${ }^{12}$. Dort, wo sich innerhalb eines Radius von $15 \mathrm{~m}$ weniger als 6 Bäume befinden, findet also ein Wechsel von variablen zu festen Probekreisen statt, $r_{i}$ ist somit immer $\leq 15 \mathrm{~m}$. Die Stammzahl pro Probekreis wird damit zur Variablen, bezeichnet mit $n_{i}$ $\left(0 \leq n_{i} \leq 6\right)$. Bei größeren Blößen ist es natürlich sinnvoll, diese vor der Inventur (z. B. mit Hilfe eines Luftbildes) zu ermitteln und bei der Stichprobennahme unberücksichtigt zu lassen. Dies beschleunigt die Meßarbeiten erheblich und kann den Stichprobenfehler deutlich reduzieren.

Nach diesen Änderungen an der Definition der Stichprobeneinheit lauten die Schätzer für die Stammzahl und Grundfläche pro ha nun

$$
\begin{aligned}
& \hat{N}_{h a}=\frac{10000}{\pi \cdot n} \cdot \sum_{\mathrm{i}=1}^{\mathrm{n}} \frac{n_{i}^{*}}{r_{i}^{2}}+\frac{n_{N B, i}}{r_{N B, i}^{2}} \text { und } \\
& \hat{G}_{h a}=\frac{10000}{\pi \cdot n} \cdot \sum_{\mathrm{i}=1}^{\mathrm{n}} \frac{n_{i}^{*}}{r_{i}^{2}} \cdot \bar{g}_{i}+\frac{n_{N B, i}}{r_{N B, i}^{2}} \cdot \bar{g}_{N B, i}, \\
& \text { mit } n_{i}^{*}= \begin{cases}n_{i} & \text { falls } n_{i}<6 \\
\frac{5}{0,9246+1,3586 \cdot \hat{W}_{P}^{3,1094}} & \text { falls } n_{i}=6\end{cases} \\
& \bar{g}_{i}=\frac{\pi}{40000 \cdot n_{i}} \cdot \sum_{j=1}^{n_{i}} d_{i j}^{2} \\
& \bar{g}_{N B, i}=\frac{\pi}{40000} \cdot d g_{N B, i}^{2}
\end{aligned}
$$

Sie erlauben in stammzahlarmen Beständen einen fließenden Übergang von der reinen 6Baum-Stichprobe zu einer konventionellen Probekreis-Inventur. Bei der Schätzung des Winkelmaßes $W_{P}$ gemäß Formel (3) bleiben neben den Probekreisen, die den Bestandesrand schneiden, nun natürlich auch die Probekreise mit weniger als 6 Bäumen unberücksichtigt.

\footnotetext{
11 Zur Kalibrierung und Kontrolle der Schätzung sollten allerdings in regelmäßigen Abständen einige Bäume gekluppt und Grenzstämme durch Abstandsmessung kontrolliert werden.

12 In der Baden-Württembergischen temporären Betriebsinventur ist der Radius der 6-Baum-Stichprobe auf 12 $\mathrm{m}$ begrenzt.
} 


\section{Validierung}

Im folgenden soll die Güte der entwickelten Schätzer für die Stammzahl und Grundfläche pro ha überprüft werden. Hierfür kam wieder die Stichprobensimulation zum Einsatz, wobei als Datenbasis 27 vollaufgenommene Bestände zur Verfügung standen, die von Plantagen mit sehr regelmäßiger Baumverteilung bis zu Plenterwäldern ein weites Spektrum von Bestandesstrukturen abdecken. Um die Spannweite der Baumverteilungsmuster weiter zu erhöhen und damit allgemeingültigere Interpretationen zu ermöglichen, wurde zusätzlich jeder Bestand dupliziert und anschließend virtuell „durchforstet“, indem alle Bäume entfernt wurden, die sich innerhalb von zufällig im Bestand verteilten Kreisflächen mit einem Radius von $10 \mathrm{~m}$ befanden (s. Abb. 6). Die Anzahl der als Femel- oder Störungsflächen interpretierbaren Entnahmeplots je Bestand wurde dabei durch eine zwischen 5 und 20 gleichverteilte Zufallszahl bestimmt.
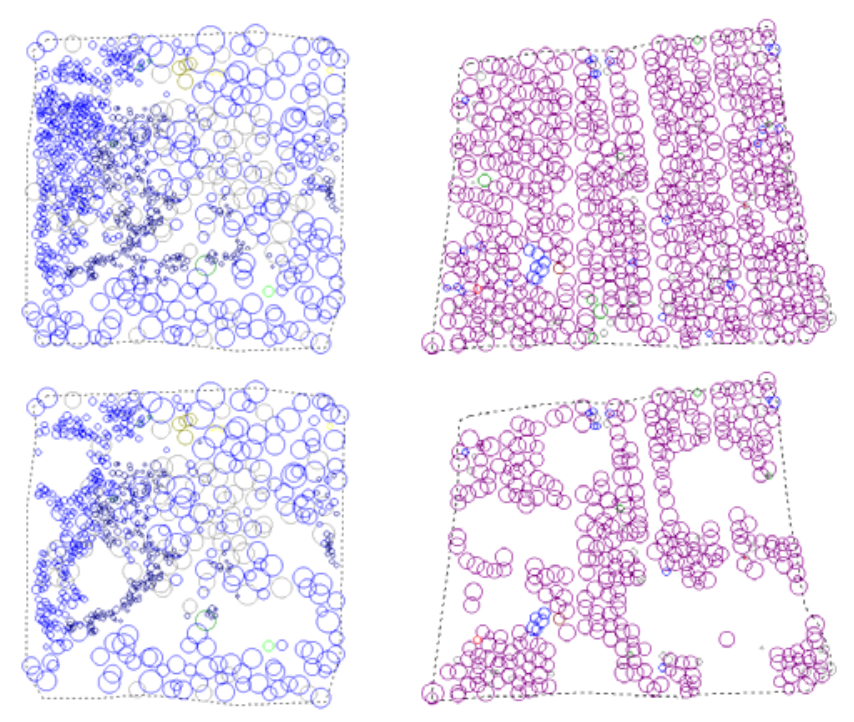

Abb. 6. Vier Beispiele der insgesamt 54 zur Validierung verwendeten Bestände mit schematischer Darstellung der Kronenschirmflächen. Links ein Plenterbestand mit großer Dimensions- und Strukturvielfalt, rechts ein fast reiner, gleichaltriger Douglasienbestand mit noch erkennbaren Pflanzreihen. Die obere Zeile zeigt den Originaldatensatz, die untere die Duplikate nach der virtuellen Durchforstung.

Four examples of the total of 54 stands, which were used for validation purposes, with schematic representation of the projected crown areas. On the left a Plenter-stand with a great variety in tree dimensions and spatial structures, on the right an almost pure, even-aged Douglas fir stand with still identifiable plant rows. The upper row shows the original data sets, the lower one the duplicated ones after the artificial thinning.

Für die Validierung standen somit insgesamt 54 Bestandesdatensätze zur Verfügung. Den dadurch abgedeckten Wertebereich der zu schätzenden Zielgrößen und des Winkelmaßes als Indikator für das räumliche Verteilungsmuster zeigt Abb. 7. Die Häufigkeitsverteilung der $W_{P}$-Werte zeigt, daß trotz der Erzeugung von Bestandeslücken und damit von zusätzlicher Klumpung kaum Werte über 0,5 erreicht werden, ein im Vergleich mit der (künstlich generierten) Datenbasis des Biasmodells sehr geringer Klumpungswert (vgl. Abb. 4). Dies deutet 
darauf hin, daß in älteren, bewirtschafteten Beständen extreme Klumpung ein äußerst seltenes Phänomen darstellen dürfte.
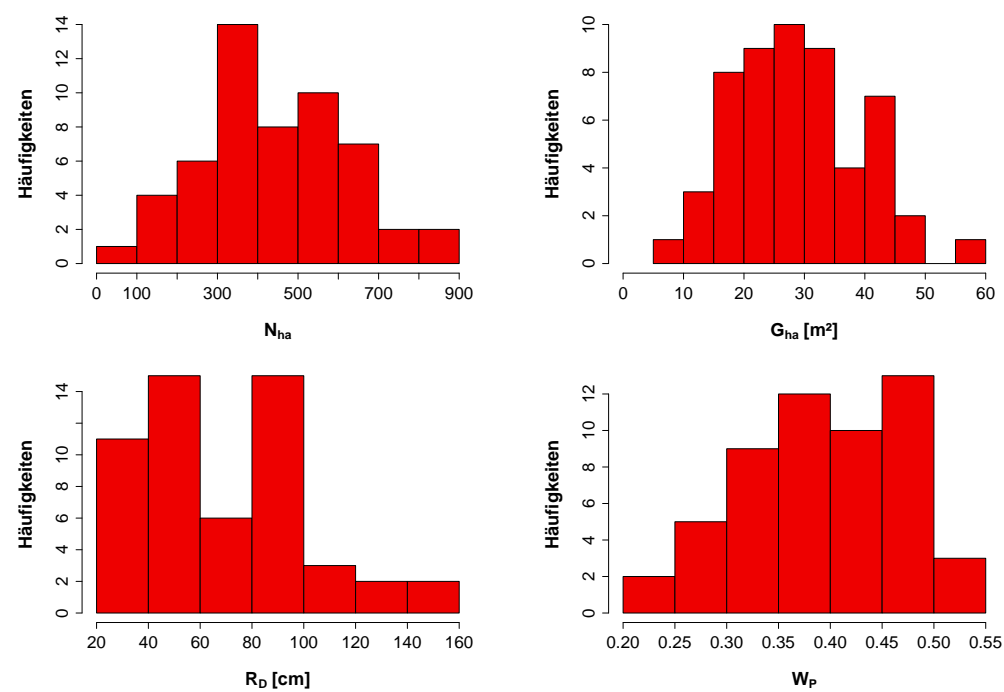

Abb. 7. Häufigkeitsverteilung der Stammzahl und Grundfläche pro ha $\left(N_{h a}\right.$ bzw. $\left.G_{h a}\right)$, der DurchmesserSpannweite $\left(R_{D}\right)$ und des punktbezogenen Winkelmaßes $\left(W_{P}\right)$ in den 54 Validierungsdatensätzen.

Frequency distribution of the stem numbers and basal areas per ha ( $N_{h a}$ and $G_{h a}$, resp.), the diameter ranges $\left(R_{D}\right)$ und the point-based Winkelmaß values $\left(W_{P}\right)$ within the 54 data sets used in the validation.

Um die durch die stufenweisen Modifikationen des Verfahrens vermutete Verbesserung der Schätzgenauigkeit überprüfen zu können, wurden alle drei hier behandelten Varianten der 6Baum-Stichprobe untersucht:

Variante I: Konventionelle 6-Baum-Stichprobe ohne Biaskorrektur (Schätzer (5) und (6) mit $k f=1$ )

Variante II: Konventionelle 6-Baum-Stichprobe mit Biaskorrektur (Schätzer (5) und (6))

Variante III: Modifizierte 6-Baum-Stichprobe mit Biaskorrektur, begrenztem Probekreisradius und nach Bestandesschichten differenzierter Datenerhebung (Schätzer (7) und (8))

Für jede dieser Varianten wurden in jedem der Validierungsbestände mit Hilfe des Stichprobensimulators PoiSSim Stichproben mit 10, 20, 30, 40 und 50 systematisch verteilten Probepunkten simuliert, mit jeweils 1000 Wiederholungen (zur Korrektur der Randfehler s. Abschnitte 3.2 u. 3.3). Da die Validierungsdaten keine Informationen über die Zugehörigkeit der Einzelbäume zu Bestandesschichten enthielten, wurde in allen Beständen mit bimodaler oder exponentieller Durchmesserverteilung ( $85 \%$ aller Validierungsdatensätze) die in Variante III vorgesehene schichtenabhängige Stratifizierung durch einen bestandesindividuellen Grenzdurchmesser vorgenommen, oberhalb dessen ein Baum zum Hauptbestand und unterhalb dessen er zum Nebenbestand (Unterstand) zählt. Die Bestimmung dieses Durchmessers erfolgte gutachtlich anhand der Durchmesserverteilung; bei bimodalen Verteilungen entsprach er dem Minimum zwischen den beiden Häufigkeitsmaxima (vgl. a. HESSENMÖLLER und GADOW 
2001), bei exponentiellen Verteilungen dem Durchmesser, bei dem die Abnahme der KlassenHäufigkeit deutlich abflacht, also beim Übergang vom Schwach- zum Mittelholz (vgl. SCHÜTZ 2002). Über alle stratifizierten Bestände beträgt er zwischen $14 \mathrm{~cm}$ und $32 \mathrm{~cm}$, im Mittel $19 \mathrm{~cm}$. Um den bei der okularen Schätzung des Mitteldurchmessers und der Stammzahl des Unterstandes zu erwartenden Fehler zu simulieren, wurde den entsprechenden Werten bei jeder Stichprobenziehung ein normalverteilter Streuungsbetrag hinzugefügt, so daß sich ein mittlerer zufälliger Fehler von $\pm 20 \%$ ergab.

Zusätzlich zum Bias wurden für jede Zielgröße auch die Standardabweichung der Stichprobenmittelwerte (= Standardfehler) und die Wurzel aus der mittleren quadratischen Abweichung der Stichprobenmittelwerte vom wahren Populationswert (Root Mean Square Error = $R M S E$ ) berechnet. Die Standardabweichung eines Parameterschätzers $\hat{\theta}$ als Maß für seine Präzision läßt sich aus $m$ Simulationsläufen schätzen über $S D(\hat{\theta})=\sqrt{E\left(\hat{\theta}_{r}-E\left(\hat{\theta}_{r}\right)\right)^{2}}$, mit $r=1 . . m$, wobei mit $m=1000$ eine ausreichend genaue Schätzung erreicht wird. Gleiches gilt für den RMSE, der auch den systematischen Fehler berücksichtigt und damit die Genauigkeit des Schätzers angibt: $\operatorname{RMSE}(\hat{\theta})=\sqrt{E\left(\hat{\theta}_{r}-E\left(\hat{\theta}_{r}\right)\right)^{2}+\operatorname{Bias}(\hat{\theta})^{2}}$. Beide Statistiken wurden durch Division durch den wahren Populationswert $\theta$ standardisiert und werden im folgenden mit $r S D$ bzw. $r R M S E$ bezeichnet.

Abb. 8 skizziert mit Box-Whisker-Plots die Verteilung der Biaswerte der Stammzahlund Grundflächenschätzung innerhalb der drei SEBAST-Varianten. Deutlich erkennbar ist die von Variante I zu Variante III abnehmende Fehlerstreuung, die im Fall der Grundflächenschätzung aber nicht nur positiv zu bewerten ist, da es dort im Mittel bei allen drei Varianten zu einer Unterschätzung kommt. Diese unterschätzende Tendenz kann erst in Variante III durch die nach Schichten getrennte Datenerhebung reduziert werden. In Variante I gibt es offensichtlich Fälle, in denen eine Unterschätzung der mittleren Baumgrundfläche durch eine Überschätzung der Stammzahl kompensiert, z. T. auch überkompensiert wird. In Variante II, in der prinzipiell die gleichen Bäume erfaßt werden wie in Variante I, führt die Korrektur des Stammzahlbias dazu, daß diese Kompensation gedämpft wird. Der Betrag des arithmetischen Mittels des Grundflächenbias ist daher in Variante II sogar größer als in Variante I, die dort auftretenden hohen positiven Biaswerte werden allerdings zuverlässig vermieden. Wie zu erwarten war, hat der Stichprobenumfang auf diese Zusammenhänge praktisch keinen Einfluß.

Auffällig ist, daß der Bias der Stammzahlschätzung in Variante III gegenüber dem in Variante II noch einmal reduziert wird. Dies ist auch plausibel, da dort in den geringen Durchmesserklassen (mit in den Plenterbeständen hohen Stammzahlen) ein erwartungstreuer Schätzer zur Anwendung kommt. Es kann jedoch festgehalten werden, daß der Bias der Stammzahlschätzung bereits allein durch den winkelmaßbasierten Korrekturfaktor in fast allen Fällen auf ein vertretbares Maß reduziert werden konnte, während für eine ausreichend 
unverzerrte Schätzung der Grundfläche zusätzlich die nach Schichten getrennt Erfassung des Bestandes notwendig zu sein scheint.
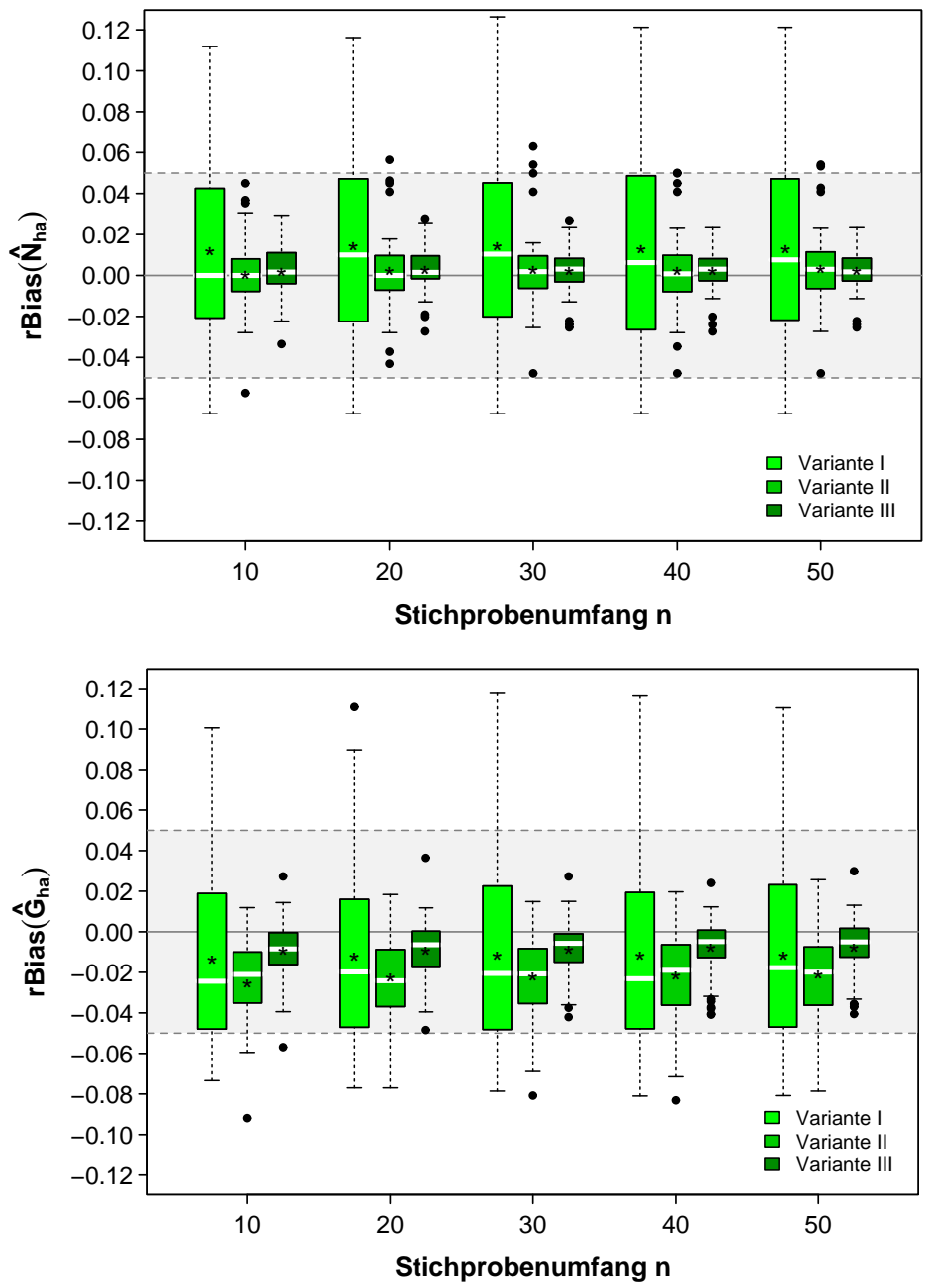

Abb. 8. Box-Whisker-Plots des relativen Bias der Stammzahl- (obere Grafik) und Grundflächenschätzung (untere Grafik) bei Anwendung der 6-Baum-Stichprobe in den drei beschriebenen Varianten mit 10 - 50 Probepunkten. Randwerte, die weiter als das 1,5-fache des Interquartilabstands vom 1. bzw. 3 Quartil entfernt sind, sind als Punkte und damit als Ausreißer dargestellt. Der Median ist durch den weißen Querstrich, das arithmetische Mittel durch einen sternförmigen Punkt angegeben.

Box-Whisker-Plots of the relative bias of the number of stems (upper panel) and basal area (lower panel), estimated with the six-tree-sample in the three described variants, with 10 - 50 sample plots. Marginal values, which are more then 1.5 times the interquartile range from the box, are shown as points and thus as outliers. The median is indicated by a white line in the middle of the box, whereas the arithmetic mean is represented by an asterix.

Abb. 9 ermöglicht eine detailliertere Betrachtung der Biaskorrektur. Sie zeigt die Werte, um die der ursprüngliche Bias der Variante I (aufgetragen auf der X-Achse) in den Varianten II und III korrigiert wurde. Wäre die Korrektur perfekt, entspräche sie exakt dem Negativwert des Bias der Variante I. Punkte ober- und unterhalb der sich daraus ergebenden Geraden indizieren eine zu starke bzw. zu schwache Korrektur. Diese Abweichungen entsprechen dem Bias, der in den Varianten II und III immer noch vorhanden oder zusätzlich entstanden ist (wenn der Abweichungsbetrag größer ist als der Betrag des Bias der Variante I). 

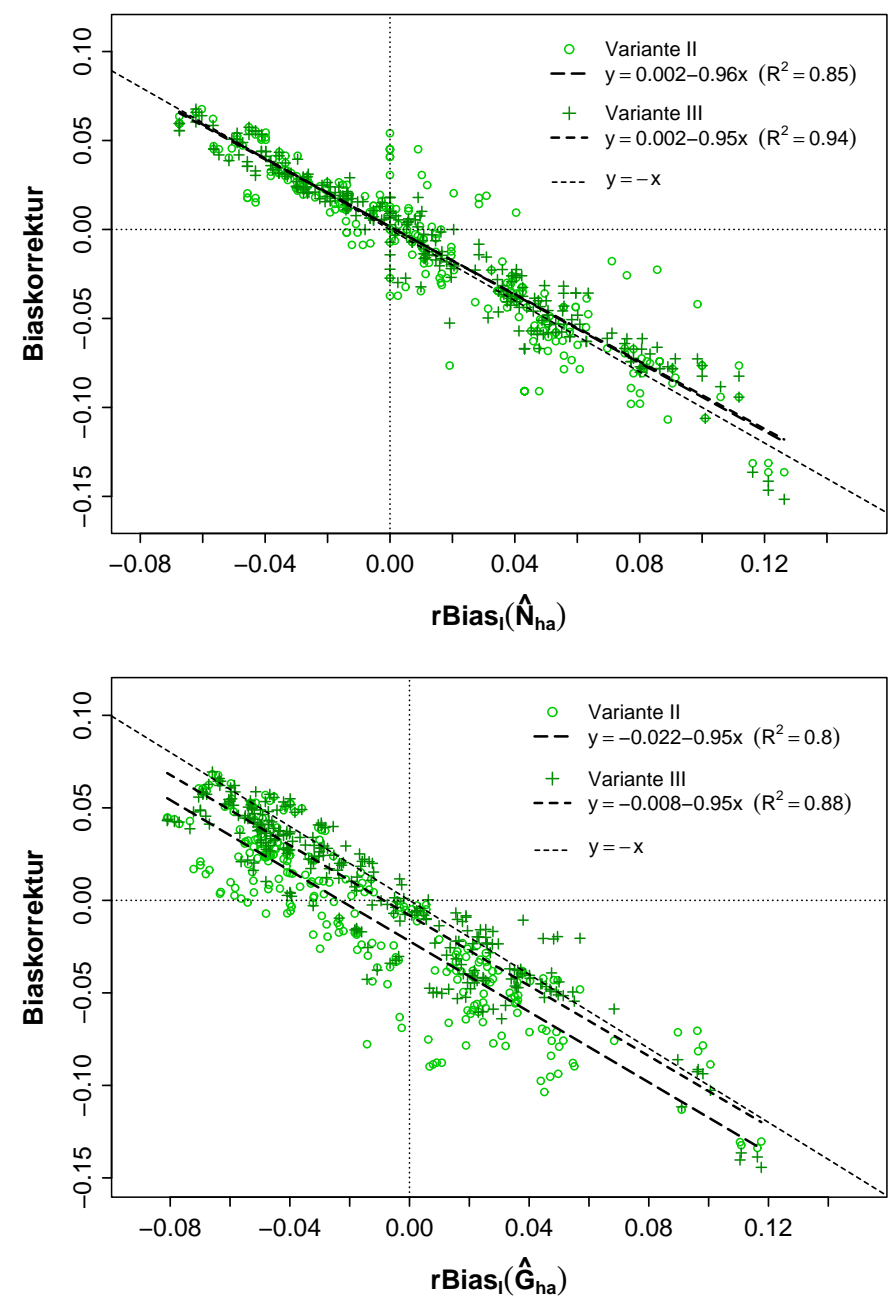

Abb. 9. Korrektur des Bias der Stammzahl- (obere Grafik) und Grundflächenschätzung (untere Grafik) in den Varianten II und III, aufgetragen über den zugehörigen Biaswerten der konventionellen SEBAST (Variante I). Die zu jeder Punktewolke gehörenden Regressionsgeraden veranschaulichen die mittleren Korrekturwerte. Idealerweise entspräche der Korrekturwert dem Negativwert des ursprünglichen Bias ( $\mathrm{y}=-\mathrm{x}$, siehe Anm. im Text).

Correction of the bias of the estimated stem number (upper panel) and basal area (lower panel) in the variants II and III against the associated bias values of the conventional six-tree-sample (variant I). The regression lines pertaining to each scatter plot illustrate the average correction values. Ideally each correction value would equal to the negative value of the original bias $(y=-x, s$. Ann. in the text).

Die Streuung der Korrekturwerte um die Idealgerade ist vor allem in Variante II, sowohl bei der Stammzahl-, als auch bei der Grundflächenschätzung, teilweise nicht unerheblich, so daß insbesondere dort, wo die konventionelle 6-Baum-Stichprobe keinen Bias aufweist (in Beständen mit Poisson-Struktur), sogar eine Verschlechterung der Erwartungstreue der Schätzungen eintreten kann. Im Fall der Stammzahlschätzung liegen die daraus resultierenden systematischen Fehler jedoch fast alle unter $5 \%$. Anders bei der Grundflächenschätzung, wo in Variante II in Beständen mit ursprünglich unverzerrter Schätzung Biaswerte von bis zu 9\% auftreten. Die fast parallel zur „Idealgeraden“ verlaufenden Regressionsgeraden, die die mittleren Korrekturwerte repräsentieren, zeigen allerdings, daß der Bias für beide Zielgrößen in beiden modifizierten Varianten im Mittel reduziert wird, andernfalls wären ihre Steigungen 
gleich 0. Die Y-Achsenabschnitte der Korrekturgeraden der Grundflächenschätzung bestätigen zudem die Erkenntnis aus Abb. 8, daß die systematische Unterschätzung der Grundfläche in Variante III gegenüber Variante II deutlich verringert wird, und zwar im Mittel um 1,4 \%.

In Abb. 10 ist der Standardfehler der Stammzahl- und Grundflächenschätzung der drei Varianten in Abhängigkeit vom Stichprobenumfang dargestellt.
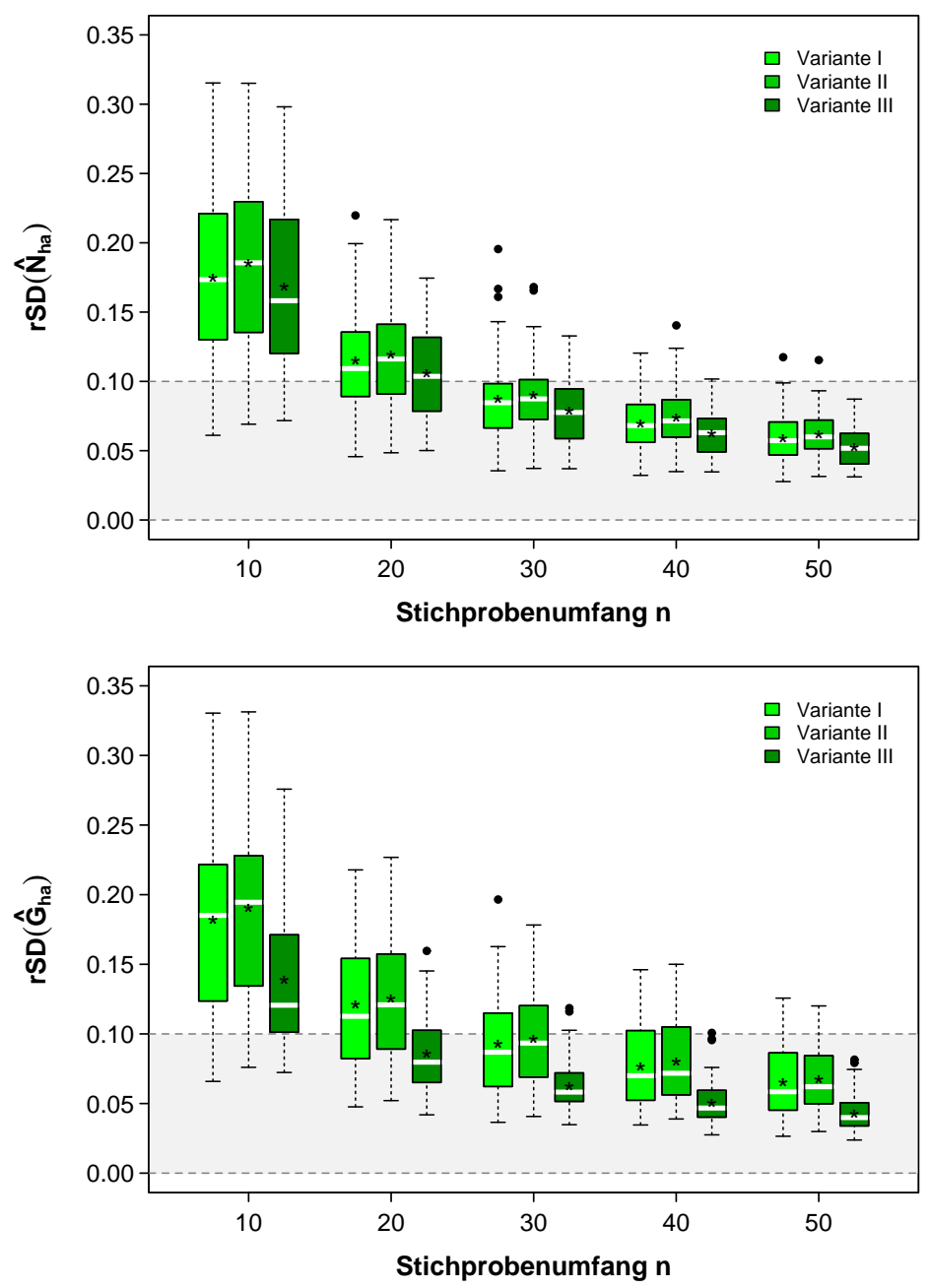

Abb. 10. Box-Whisker-Plots des relativen Standardfehlers der Stammzahl- (obere Grafik) und Grundflächenschätzung (untere Grafik) mit der 6-Baum-Stichprobe in den drei beschriebenen Varianten bei 10 bis 50 Stichprobepunkten (zur weiteren Erläuterung der Darstellung s. Abb. 8).

Box-Whisker-Plots of the relative standard errors of the stem number (upper panel) and basal area (lower panel), estimated with the six-tree-sample in the three described variants, with $10-50$ sample plots (for further explanations see fig. 8).

Erwartungsgemäß nimmt er mit zunehmendem Stichprobenumfang bei allen Varianten überproportional ab. Im Vergleich zwischen den Varianten hebt sich wiederum Variante III mit deutlich geringeren Fehlerwerten $a b$. Wie primär beabsichtigt, führt die Stratifizierung vor allem bei der Grundflächenschätzung zu einer deutlich verbesserten Präzision. Dadurch kann bereits ab 30 Probepunkten in fast allen Beständen, also selbst in den extrem heterogenen, „durchforsteten“ Plenterbeständen, ein mittlerer Stichprobenfehler von $10 \%$ eingehalten und 
unterschritten werden. Interessant ist, daß das Fehlerniveau in Variante II etwas höher liegt als in Variante I, obwohl in beiden Varianten prinzipiell die gleichen Bäume erfaßt werden. Dies läßt sich dadurch erklären, daß die Stammzahlschätzung in Variante II durch einen Korrekturfaktor modifiziert wird, dessen Wert auf der Schätzung des Winkelmaßes beruht. Die Streuung der Winkelmaßwerte zwischen den Stichproben bewirkt offensichtlich eine zusätzliche Varianz der Stammzahlschätzungen und damit einen gegenüber Variante I leicht erhöhten Standardfehler, der sich auch auf die Schätzung der Grundfläche auswirkt.

Eine genauere Quantifizierung dieser Zusammenhänge erlaubt Abb. 11, in der die Standardfehler der Varianten II und III über den Standardfehlern der Variante I aufgetragen sind.
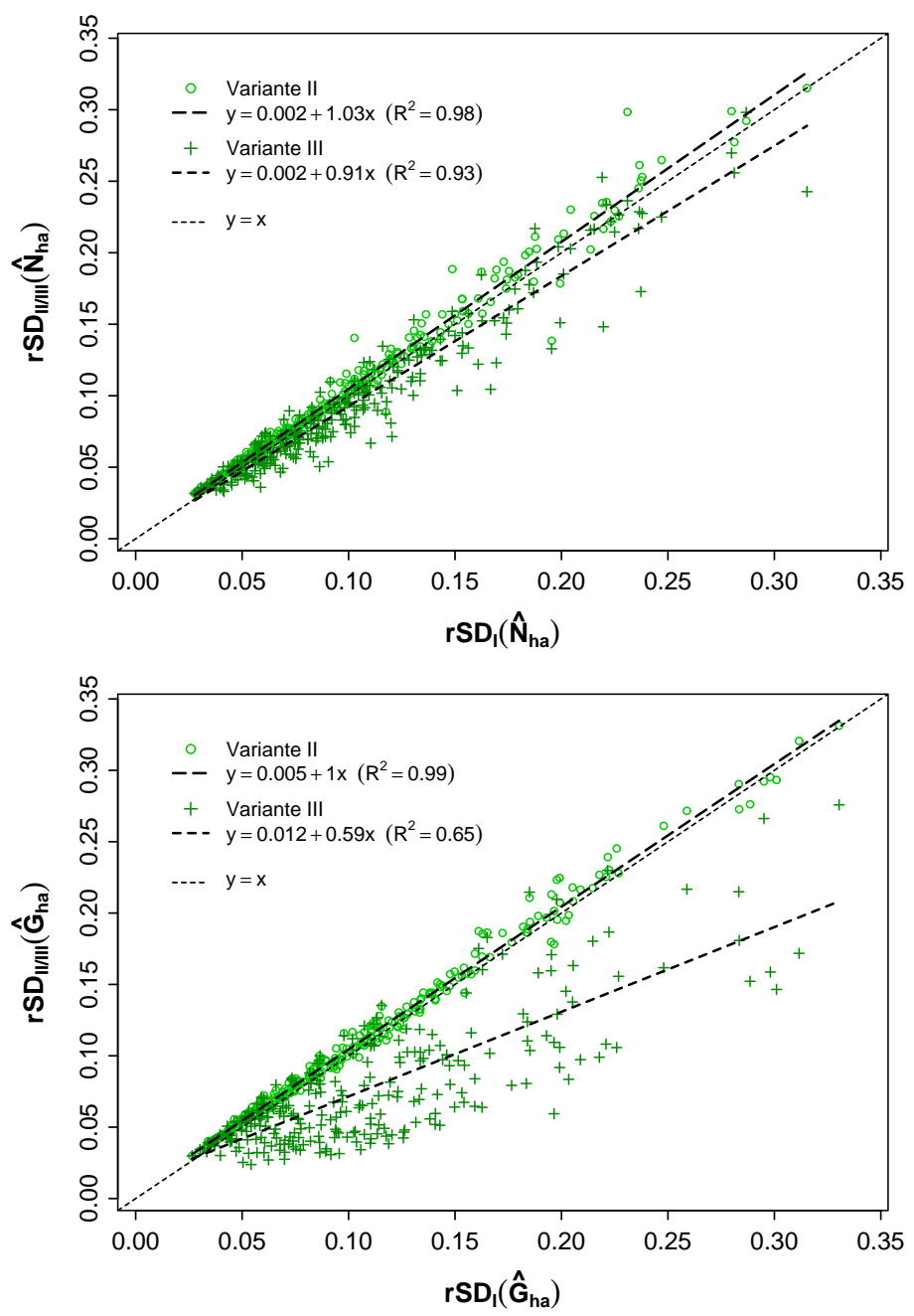

Abb. 11. Veränderung des Standardfehlers der Stammzahl- (obere Grafik) und Grundflächenschätzung (untere Grafik) bei Anwendung der modifizierten SEBAST (Varianten II und III) im Vergleich zur konventionellen SEBAST (Variante I). Um das mittlere Fehlerverhältnis zu verdeutlichen, wurde jede Punktewolke mit einer Regressionsgerade ausgeglichen.

Change of the standard error of the stem number (upper panel) and basal area (upper panel) estimation, when the modified six-tree-sample (variants II and III) is applied, against the conventional six-treesample (variant I). In order to clarify the mean error ratio, each scatter plot was smoothed with a regression line. 
Punkte oberhalb der Ursprungsgeraden zeigen eine Verschlechterung, Punkte unterhalb eine Verbesserung der Präzision der Schätzungen an. Die Koeffizienten der Regressionsgeraden durch die Punktewolken zeigen, daß der Standardfehler der Stammzahlschätzung in Variante II im Mittel mindestens 3 \% höher, in Variante III maximal $9 \%$ niedriger ist als in Variante I. Auch die Grundfläche wird in Variante II im Mittel etwas schlechter geschätzt als in Variante I (konst. $+0,5 \%$ ), in Variante III kommt es hingegen zu einer drastischen Fehlerabsenkung um bis zu $37 \%$.

Diese Fehlerreduktion ist umso beachtlicher, als in der Stichprobenziehung ein mittlerer zufälliger Fehler der okularen Schätzung des Mitteldurchmessers und der Stammzahl des Unterstandes von $20 \%$ simuliert wurde. Dieser Fehler wird aber offenbar durch die in Variante III vergrößerte Stichprobeneinheit mehr als ausgeglichen. Dies betrifft v. a. die Grundflächenschätzung, was auch zu erwarten war, da die Bäume des Hauptbestandes, die einen entsprechend hohen Anteil an der Bestandesgrundfläche einnehmen, in Variante III eine deutlich erhöhte Auswahlwahrscheinlichkeit haben und damit die Stichproben-Effizienz hinsichtlich dieses Merkmals erhöht wird.

\section{Schlussfolgerungen}

Die Ergebnisse der Validierung lassen sich wie folgt zusammenfassen:

1.) Durch die Verwendung des winkelmaßbasierten Korrekturfaktors läßt sich der Bias der Stammzahlschätzung in einem weiten Spektrum von Bestandestypen mit unterschiedlichsten räumlichen Strukturen in einem vertretbaren Rahmen von $\pm 5 \%$ halten, was zur Folge hat, daß der $r R M S E$ als Maß für den Gesamtfehler der Schätzung in $96 \%$ (Variante II) bzw. 99,8 \% (Variante III) der Fälle unterhalb des 1,1-fachen Standardfehlers bleibt. Dies ermöglicht eine universelle Anwendbarkeit der 6-Baum-Stichprobe, die nicht wie bisher (vgl. z. B. PICARD et al. 2005) durch Annahmen zum Baumverteilungsmuster eingeschränkt ist.

Eine statistisch saubere und elegantere Lösung stellt freilich der Ansatz von KLEINN und VILČKO (2006b) dar, bei dem die Einschlußflächen mit Hilfe der $k$-order Voronoi Polygone direkt berechnet werden. Auf der Basis der dann bekannten Auswahlwahrscheinlichkeiten der Stichprobenbäume läßt sich mit dem Horwitz-Thompson-Schätzer (HoRwITZ und THOMPSON 1952) jede beliebige Bestandesvariable erwartungstreu schätzen. Allerdings müssen für die Berechnung der Einschlußflächen die Positionen der Stichprobenbäume und zusätzlich - je nach Raumstruktur - evtl. zahlreicher weiterer benachbarter Bäume eingemessen werden. Dies kann, v. a. in dichten Beständen mit Unterstand, selbst mit Laserentfernungsmessern eine aufwendige Prozedur sein, die der Schnelligkeit des Verfahrens zuwiderläuft. Solange diese inventurtechnischen Fragen nicht geklärt sind, könnte also der hier beschriebene Ansatz eine wertvolle Alternative sein. 
2.) Durch die Beschränkung der eigentlichen (bias-korrigierten) 6-Baum-Stichprobe auf den Hauptbestand und die Erfassung des Nebenbestandes mittels okularer Schätzung von Mitteldurchmesser und Stammzahl innerhalb fester Probekreise kann auch der Betrag des Bias der Grundflächenschätzung selbst in extrem strukturreichen Beständen auf ein vertretbares $\mathrm{Maß}$ von unter $5 \%$ gesenkt werden (der $r R M S E$ beträgt dadurch immerhin noch in $90 \%$ der Fälle weniger als das 1,1-fache des Standardfehlers). Während der Stichprobenfehler in Variante II im Mittel sogar etwas höher war als in der ursprünglichen 6-Baum-Stichprobe, was durch den Einfluß der Streuung der Winkelmaßes erklärt werden kann, führt die Vergrößerung der bei der konventionellen 6-Baum-Stichprobe grenzwertig kleinen Stichprobeneinheit ${ }^{13}$ in Variante III zu einer deutlichen Verringerung des Stichprobenfehlers, vor allem bei der Schätzung der Grundfläche pro ha, der in den meisten Fällen wohl wichtigsten meßbaren Variable bei Bestandesinventuren. Auch der Bias der Stammzahlschätzung wird weiter reduziert. Dem gegenüber ist der Mehraufwand im Vergleich zur konventionellen 6-BaumStichprobe, bedingt durch die stratenspezifische Genauigkeit der Datenerhebung, sehr gering.

3.) Durch die Begrenzung des Radius der SEBAST-Probekreise auf maximal $15 \mathrm{~m}$ wird die Einfachheit der Datenerfassung im Gelände weiter erhöht. Eine dadurch bedingte Verschlechterung der Schätzgenauigkeit, im Sinne eines systematischen Fehlers, konnte in dieser Untersuchung nicht nachgewiesen werden.

Um die tatsächliche Effizienz des hier vorgestellten Verfahrens im Vergleich zu konkurrierenden Inventurmethoden besser beurteilen zu können, wären Zeitstudien natürlich sehr wünschenswert gewesen. Ebenso wäre es sinnvoll, zu untersuchen, welche Auswirkungen ein systematischer Fehler bei der okularen Schätzung des Unterstandes auf die Schätzung der Stammzahl und Grundfläche des Gesamtbestandes hat. Die Bearbeitung dieser Fragen hätte jedoch den Rahmen dieser Arbeit gesprengt und bleibt somit zukünftigen Untersuchungen vorbehalten.

\section{Danksagung}

Der Autor sagt herzlichen Dank an Prof. Dr. Klaus v. Gadow, Dr. Matthias Albert, Institut für Waldinventur und Waldwachstum, Universität Göttingen, und Dr. Matthias Schmidt, Nordwestdeutsche Forstliche Versuchsanstalt, Göttingen, für zahlreiche, wertvolle Diskussionen und Verbesserungsvorschläge. Besonders danken möchte ich auch Prof. Dr. Walter Zucchini, Institut für Statistik und Ökonometrie, Universität Göttingen, und Prof. Dr. Joachim Saborowski, Institut für Forstliche Biometrie und Informatik, Universität Göttingen, für ihre wertvolle Hilfe in statistischen Fragen. Ebenso danke ich zwei anonymen Gutachtern für ihre

13 Die optimale Größe der Stichprobeneinheit wird von AKÇA (2001, S. 38) für deutsche Verhältnisse mit 15 - 20 Bäumen angegeben. 
gründliche Durchsicht des Manuskripts und substantielle Hinweise, Korrekturen und Verbesserungsvorschläge. Andreas Zingg, Eidg. Forschungsanstalt für Wald, Schnee und Landschaft (WSL), Birmensdorf, sei vielmals gedankt für die Erlaubnis, Daten von schweizerischen Plenterwald-Versuchsflächen zu nutzen. Diese Arbeit wurde finanziert durch die Deutsche Forschungsgemeinschaft (Förderprojekt Ga 473/11-2), wofür ich ebenfalls danken möchte.

\section{Literatur}

AKÇA, A., 2001: Waldinventur. J. D. Sauerländer's, Frankfurt a. M., 193 S.

BAdDeley, A., TURner, R., with contributions by Lieshout, M.-C. V., and 17 others, 2006: Spatstat: Spatial point pattern analysis, model-fitting and simulation. $\mathrm{R}$ package version 1.8-5, Internet: http://www.spatstat.org.

BEERS, T.W., 1966: The direct correction of boundery-line slopover in horizontal point sampling. Purdue Univ., Agri. Exp. Sta., Lafayette, Ind. Res. Progr. Rep. 224, 8 pp.

BitTERLiCH, W., 1948: Die Winkelzählprobe. Allg. Forst- u. Holzwirtsch. Ztg. 59 (1/2): 4 - 5.

Blust, M., Korn-Allan, E., und Von Der Goltz, H., 2006: Nachhaltigkeitsmonitoring mittels Betriebsinventur. AFZ/Der Wald 61 (9): 469 - 470.

BYTH, K., and RIPLEY, B.D., 1980: On sampling spatial patterns by distance methods. Biometrics 36: 279 - 284.

Cox, F., 1971: Dichtebestimmung und Strukturanalyse von Pflanzenpopulationen mit Hilfe von Abstandsmessungen. Mitt. d. Bundesforsch.anst. f. Forst- und Holzwirtsch. Reinbek Nr. 67, $161 \mathrm{~S}$.

Diggle, P.J., BeSAG, J.E., and GlaVES, J.T., 1976: Statistical analysis of spatial point patterns by means of distance methods. Biometrics 32: 659 - 667.

GAdow, K. V., Hui, G. Y., und AlBert, M., 1998: Das Winkelmaß - ein Strukturparameter zur Beschreibung der Individualverteilung in Waldbeständen. Centralbl. f. d. ges. Forstw. 115: 1 - 10.

Gleichmar, W., und Gerold, D., 1998: Indizes zur Charakterisierung der horizontalen Baumverteilung. Forstw. Cbl. 117: 69 - 80.

HessenMÖLleR, D., und GADOw, K. v., 2001: Beschreibung der Durchmesserverteilung von Buchenbeständen mit Hilfe der bimodalen Weibullfunktion. Allg. Forst- u. Jagdzeitg. 172 (3): 46 - 50.

HIRNER, V., 1978: Theoretische Überlegungen zur Sechs-Baum-Stichprobe und deren praktische Anwendungsmöglichkeiten. Diss., Forstwiss. Fak., Univ. Freiburg (Breisgau), 114 S.

Horwitz, D., and Thompson, D., 1952: A Generalisation of Sampling without Replacement from a finite Universe“, J. Amer. Stat. Ass. 47: 663 - 685.

Husch, B., Miller, C.I, and BeERS, T.W., 1993: Forest Mensuration. Krieger, 402 pp.

Jonsson, B., Holm, S., and KalluR, H., 1992: A forest inventory method based on density-adapted circular plot size. Scand. J. For. Res. 7: 405 - 421.

KLEINN, CH., und VILČKO, F., 2005: Ein Vergleich von zwei methodischen Konzepten für die Grundgesamtheit von Probeflächen bei Waldinventuren. Allg. Forst- u. Jagdzeitg. 176: 68 - 74.

Kleinn, CH., and ViLČKO, F., 2006a: A new empirical approach for estimation in k-tree sampling. For. Ecol. Managm. 237 (2): 522 - 533.

KleINN, CH., and VILČKO, F., 2006b: Design unbiased estimation for point-to-tree distance sampling. Can. J. For. Res. 36 (6): 1407 - 1414.

Ko, Thiry, Pelz und ErlensPiel, 1969: 6-Baum-Stichprobe für die Forsteinrichtung. Allg. Forst- u. Jagdzeitg. 140 (8): $186-189$. 
KÖNIG, G., 1835: Die Forstmathematik mit Anweisung zur Forstvermessung, Holzschätzung und Waldwerthrechnung, nebst Hülfstafeln für Forstschätzer. Gotha, in Commision der Becker'schen Buchhandlung. $\mathrm{X}, 436,56 \mathrm{~S}$.

Lessard, V., ReED, D.D., and Monkevich, N., 1994: Comparing N-tree distance sampling with point and plot sampling in northern Michigan forest types. North. J. Appl. For. 11 (1): 12 - 16.

LESSARD, V., Drummer, T.D., and Reed, D.D., 2002: Precision of Density Estimates from Fixed-Radius Plots Compared to N-Tree Distance Sampling. Forest Science 48 (1): 1 - 6.

Loetsch, F, ZÖHrer, F., and Haller, K.E., 1973: Forest Inventory, Vol. II. BLV Verlagsgesellschaft, München, $469 \mathrm{pp}$.

NIEDERSÄCHSISCHE LANDESFORSTEN, 1987: Anweisung zur Betriebsregelung (Forsteinrichtung) in den Niedersächsischen Landesforsten. Nieders. Minist. f. Ernähr., Landwirtsch. u. Forsten, 55 S.

Okabe, A., Boots, B., Sugihara, K., and Chiu, S.N., 1999: Spatial Tesselation - Concepts and Applications of Voronoi Diagrams. 2nd Ed., Wiley, $671 \mathrm{pp}$.

PAYANDEh, B., and EK, A.R., 1986: Distance methods and density estimators. Can. J. For. Res. 16: 918 - 924.

PiCARD, N., Kouyaté, A.M., and Dessard, H., 2005: Tree density estimation using a distance method in Mali Savanna. For. Sci. 51 (1): 7 - 18.

PIELOU, E.C., 1977: Mathematical Ecology. Wiley, New York. 385 pp.

Pommerening, A., 1997: Eine Analyse neuer Ansätze zur Bestandesinventur in strukturreichen Wäldern. Göttingen, Univ., Diss., $150 \mathrm{~S}$.

Prodan, M., 1968: Punktstichprobe für die Forsteinrichtung. Der Forst- u. Holzwirt 23: 225 - 226.

R Development Core TeAm, 2005: R: A language and environment for statistical computing. R Foundation for Statistical Computing, Vienna, Austria. ISBN 3-900051-07-0, Internet: http://www.R-project.org.

RIPLEY, B.D., 1981: Spatial Statistics. John Wiley \& Sons Inc., New York, 252 pp.

SABOROWSKI, J. und ŠMELKO, S., 1998: Zur Auswertung von Stichprobeninventuren mit variablen Probeflächengrößen. Allg. Forst- u. Jagdzeitg. 169 (4): 71 - 75.

SCHMID-HAAS, P., 1969: Stichproben am Waldrand. Mitt. der Eidgen. Anstalt. Forstl. Vers. 45: 233 - 303.

SchÖPFER, W., 1969a: Die 6-Baum-Stichprobe in der Forsteinrichtung (Teil 1). Allg. Forst Zeitg. $25: 533$ - 536.

SCHÖPFER, W., 1969b: Die 6-Baum-Stichprobe in der Forsteinrichtung (Teil 2). Allg. Forst Zeitg. $26: 588$ - 591.

SCHÜTZ, J.-PH., 2002: Die Plenterung und ihre unterschiedlichen Formen - Skript zu Vorlesung Waldbau II und Waldbau IV. Professur Waldbau, ETH Zürich, $126 \mathrm{~S}$.

Sheil, D., Ducey, M.J., and SAmsoedin, I., 2003: A new type of sample unit for the efficient assessment of diverse tree communities in complex forest landscapes. J. Trop. For. Sci. 15 (1): 117 - 135.

SLOBODA, B., 1976: Mathematische und stochastische Modelle zur Beschreibung der Statik und Dynamik von Bäumen und Beständen. Habil., Forstwiss. Fak., Univ. Freiburg (Breisgau).

SMAltschinski, TH., 1981: Dichte und Bestandesstruktur. Diss., Forstwiss. Fak., Univ. Freiburg (i. Br.), 127 S.

SMALTSCHINSKI, TH., 1998: Charakterisierung von Baumverteilungen. Forstw. Cbl. 117: 355 - 361.

StAGE, A.R., and RENNIE, J.C., 1994: Fixed-radius plots or variable-radius plots? Journal of Forestry 92 (12): $20-24$.

STARKE, J., 1989: Kontrollstichproben in Niedersachsen. Forst u. Holz 44 (13): 331 - 341.

StAuPENDAHL, K., 2001: PoiSSim - Ein Programm zur Simulation von Punktstichproben. ARGUS Forstplanung, Göttingen.

StAupendahl, K., and ZuCCHINI, W., 2006: Estimating the spatial distribution in forest stands by counting small angles between nearest neighbours, Allg. Forst- u. Jagdzeitg. 177 (8/9): 160 - 168.

Stoffels, A., 1955: Die Genauigkeit der Bestimmung der Stammzahl pro Hektar durch Messung von Stammabständen. Forstwiss. Centralbl. 74: 211 - 218. 
Stoyan, D., and Stoyan, H., 1992: Fraktale, Formen, Punktfelder: Methoden der Geometrie-Statistik. Berlin, Akad.-Verl., $394 \mathrm{~S}$.

Stoyan, D., 1995: Stochastic Geometry 4.1 - ein Windows-Programm zur Erzeugung, Darstellung und statistischen Auswertung verschiedener Modelle der ebenen Stochastischen Geometrie.

Upton, G., and Fingleton, B., 1985: Spatial Data Analysis by Example. Vol. 2: Categorical and Directional Data. John Wiley \& Sons. 416 pp.

Upton, G., and Fingleton, B., 1989: Spatial Data Analysis by Example. Vol. 1: Point Pattern and Quantitative Data. John Wiley \& Sons. 410 pp.

UTSCHIG, H., 2002: Analyse der Standraumökonomie von Einzelbäumen auf langfristig beobachteten Versuchsflächen - Methoden, Programmentwicklung und erste Ergebnisse, Forstw. Cbl. 121(6): S. 335 - 348

Venables, W.N., and RIPLEY, B.D., 2002: Modern Applied Statistics with S. Fourth Edition. Springer, New York, $495 \mathrm{pp}$.

VILČKO, F., 2006: Selected research issues in fixed count sampling. PhD colloquium at the Institute of Forest Management and Yield Science, Georg-August-Univ., Göttingen (11.05.2006). 


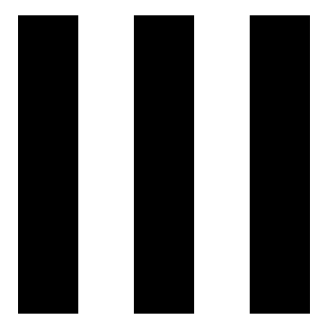





\title{
Estimating the spatial distribution in forest stands by counting small angles between nearest neighbours
}

\section{Beurteilung des räumlichen Verteilungsmusters in Waldbeständen anhand der Anzahl kleiner Winkel zwischen benachbarten Bäumen}

\author{
KAI STAUPENDAHL ${ }^{1}$ and WALTER ZUCCHINI ${ }^{2}$
}

Allgemeine Forst- und Jagdzeitung 177 (8/9): 160 - 168

Schlagwörter: Abstandsverfahren, Strukturindizes, räumliche Verteilungsmuster, räumliche Zufälligkeit, Test auf Zufälligkeit.

\section{Zusammenfassung}

Seit geraumer Zeit sind Methoden zur Beschreibung der Struktur von Waldbeständen und -landschaften ein wesentlicher Bestandteil der forstlichen Forschung. In dieser Arbeit werden die Eigenschaften eines Index' untersucht, der das räumliche Verteilungsmuster der Bäume in einem Bestand beschreibt und auf folgender Idee beruht: Betrachtet man die von einem gegebenen Punkt aus gesehen drei nächsten Bäume und zählt die Winkel zwischen ihnen, die kleiner sind als ein vorher festgelegter kritischer Winkel, so beschreibt der Anteil dieser kleinen Winkel den Grad der Klumpung. Praktische Tests haben gezeigt, daß die Methode einfach und schnell anzuwenden und damit potentiell kosteneffizient ist. Es wird ein auf diesem Index beruhender Test vorgestellt, mit dem die Hypothese der vollständigen räumlichen Zufälligkeit überprüft werden kann. Untersuchungen zur Teststärke zeigen, daß dessen Güte mit der Güte alternativer Tests vergleichbar ist, bei geringerem Meßaufwand. Darüber hinaus ergaben Simulationsstudien, daß der Erwartungswert und Standardfehler des Index' verzerrungsfrei geschätzt werden können.

Key words: Distance methods; six-tree-sample; bias; spatial patterns; stand inventory.

\begin{abstract}
Methods for describing the structure of a tree population have been used for a considerable time as an integral part of forest research. In this paper we investigate the properties of a sim-

\footnotetext{
1 Dipl.-Forstw. Kai Staupendahl (corresponding author), ARGUS Forstplanung, Büsgenweg 5, D-37077 Göttingen, Telephone: +49-551-39 34 76, Fax:+49-551-39 57 67, e-mail: staupendahl@argus-forstplanung.de

2 Prof. Dr. Walter Zucchini, Institute of Statistics and Econometrics, Faculty of Economic Sciences, GeorgAugust-University Göttingen, Platz der Göttinger Sieben 5, D-37073 Göttingen, Telephone: +49-551-39 72 86, e-mail: walter.zucchini@wi-wiss.uni-goettingen.de
} 
ple measure to describe the spatial pattern of tree positions, based on the following idea: The three angles between the three nearest trees from a given reference point are each classified as being either "large" or "small", where small is defined as being smaller than some predetermined critical angle. The measure is simply the proportion of small angles out of three. Practical tests have shown that the method is easy to apply and is thus potentially cost-effective. We investigate the power of a test that is based on this measure to test the hypothesis of complete spatial randomness. We show that its performance is comparable to alternative methods that are more costly to implement. It is also shown that the expected value and standard error of the measure can be estimated unbiasedly.

\section{Introduction}

\subsection{The importance of forest structure}

Much has been written about sustainable forest management during the past 200 years. The more recent discussions concentrate, among other things, on ecological, economic and social indicators describing the type of forest management and the condition of managed forests (e.g. MCPFE 2003). In this context particular emphasis is placed on indicators for assessing and describing forest structures (KÖHL and ZINGG 1995; SZARO and JOHNSTON 1996; VANCLAY 1998), since biological diversity in forests is mainly determined by the structural variety within and between different geographical units known as stands or compartments (ОTTO 1994, pp. 180; SPELLMANN 1995; KIMMINS 1997, pp. 352). Only by measuring relevant aspects of structure can one assess in how far a forest conforms to an "ideal" condition.

SCHREUDER et al. (1993, pp. 341) pointed out that knowledge of spatial distributions in forests is important both from an inventory and a management point of view. For inventory purposes, spatial distribution may explain the results or facilitate the comparison of inventory methods, in terms of the joint probabilities of selection of individual trees (see e.g. STAUPENDAHL 2006). For regeneration purposes, stocking percentages and location of regeneration need to be known to ensure that stocking is adequate over the entire area, whereas in older stands, a good understanding of spatial distributions may be helpful in designing thinning schedules, optimal harvesting techniques and development of individual tree and growth models. PRETZSCH (1995), e.g., has shown that tree growth may be influenced by the spatial structure of a stand. Thus, the accuracy of distance-dependent growth models is greatly affected by the use of suitable parameters of forest spatial structure (e.g. PRETZSCH 1997; POMMERENING 1998). A practical requirement is that the assessment of such parameters be cost-effective and that they should result in a realistic reproduction of stand structures from a representative sample. In order to achieve this objective, all important aspects of structure 
have to be combined in a multi-dimensional approach (cf. LEWANDOWSKI and GADOW 1997; PRETZSCH 1997; POMMERENING 2006)

A great variety of forest structure indices were developed or adopted for forestry use from other disciplines (cf. UptON and FINGLETON 1989, 1990; BIBER 1997; GLEICHMAR and GEROLD 1998; SMALTSCHINSKI 1998; GADOW 1999). For practical forestry purposes, where complete mappings of populations are normally not feasible, the so-called distance methods are of particular importance. These methods, which are also used for intensity estimation, are based on nearest-neighbour distances, whereas the measurements made are of two basic types: distances from a sample point to a tree (Fig. 1a) or from a tree to a tree (Fig. 1b-d) ${ }^{3}$. Again from these methods the $T$-square sampling (BESAG and GLAVES 1973) and their modifications (HINES and HINES 1979) have proven to be particularly useful (DIGGLE et al. 1976; BYTH and RIPLEY 1980), with special respect to the test of complete spatial randomness.

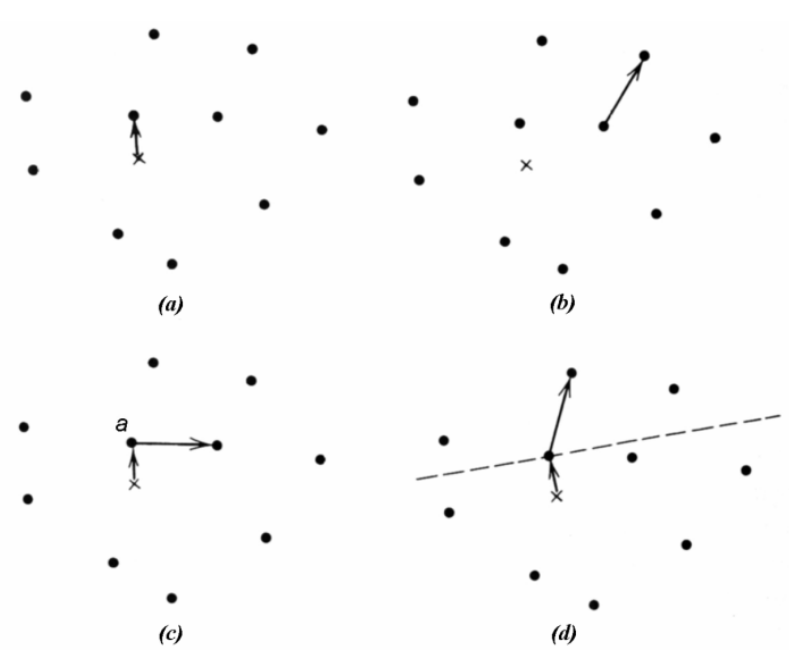

Fig. 1. Types of distance measurements ( $\bullet$ denotes a tree, $\mathbf{x}$ a randomly chosen sample point): (a) Sample point to nearest tree; (b) randomly chosen sample tree to nearest tree; (c) sample tree $a$ to nearest tree, where $a$ is the nearest tree to the randomly chosen sample point; (d) $T$-square sampling (RIPLEY 1981, p. 131). Arten der Abstandsmessung (• markiert einen Baum, $x$ einen zufällig ausgewählten Stichprobenpunkt): (a) Vom Stichprobenpunkt zum nächsten Baum; (b) vom zufällig gewählten Probebaum zum nächsten Nachbarn; (c) vom Probebaum $a$ zum nächsten Nachbarn, wobei $a$ der nächstgelegene Baum zum zufällig gewählten Stichprobenpunkt ist; (d) T-Quadrat-Stichprobe (RIPLEY 1981, S. 131).

However, in difficult terrain the distances required to evaluate the $T$-square statistics can be hard to obtain. For such situations ASSUNÇÃo (1994) proposed a method based on the angle between the vectors joining each sample point to its two nearest neighbours. With the same intention GADOW et al. (1998) presented the index $W$ which uses the angle between neighboured trees as well, but with a tree as origin instead of a point, with more than two trees being taken into consideration. However, the decisive difference to ASSUNÇãO's method is that

\footnotetext{
3 Sometimes the terms "distance methods" and "nearest neighbour methods" are used to distinguish the two types, but mostly they are regarded as synonymous.
} 
the angles are not measured exactly; it is only necessary to determine whether an angle is bigger or smaller than some specified threshold, such as the angle associated with strict regularity. In most cases this leads to a considerable reduction in the measuring effort in the field. The measure proposed in this paper tries to combine the advantage of these methods.

\subsection{Point-based versus tree-based description of forest structure}

In this paper a structure index is denoted as tree-based if it measures the structure locally around a tree, i.e. can be regarded as an attribute of the tree. In terms of distance methods this measurement is based on tree-to-tree distances; the result of the assessment is a frequency distribution of the number of stems. Clearly this is different from the case in which the structure is measured locally around a point, which involves point-to-tree distances and which is therefore regarded as a point attribute. For the latter, the relative frequencies in the resulting distribution can be interpreted as area proportions, since the sum of all points within a forest stand makes up the stand area ${ }^{4}$. Such an index is denoted as point-based.

STAUPENDAHL (1997) pointed out that, for many structural attributes of a tree population, a point-based index is more meaningful than a tree-based one. Imagine, for example, a forest stand with two separate subpopulations with equal number of trees. The trees are distributed regularly in the one subpopulation, at random in the other. Clearly the spatial structure of the entire population will differ substantially depending on whether the ratio of the fractions of the areas occupied by the two subpopulations is $1: 1$ or $1: 10$. However, a treebased description would give approximately the same structure in both cases ${ }^{5}$.

A second reason for switching to a point-based approach is that, in the tree-based approach, the sample trees have to be chosen at random (Fig. 1b). Since such a sampling design is often impractical, the nearest to the (systematically chosen) sample point is taken as the sample tree (Fig. 1c,d). The outcome of this is that the trees have different probabilities of being selected. For example the trees "near the centre" of a cluster are less likely to be chosen than those on the "outside of the cluster" (RIPLEY 1981, pp. 131). The differences in the selection probabilities increase with increasing clumping.

The Voronoi polygon associated with a given tree is defined as the set of points in the stand that are nearer to the tree than to any other tree (RIPLEY 1981, p. 38); the probability that the tree is selected is proportional to the size of its Voronoi polygon. Consequently, if the value of the tree-based structure index, or any other tree-based index, is correlated with the size of the Voronoi polygon, the resulting estimates of the index will be biased, and the bias

\footnotetext{
4 The problem of this approach is the infinite number of points in a stand, in contrast to the finite number of stems. This will be discussed later.

5 This is, by the way, the reason why the index of CLARK and EVANS (1954) can give the same value for very different distribution patterns (cf. HOPKINS and SKELLAM 1954; COX 1971).
} 
will increase with increasing clumping 6 . This explains the bias in estimating the tree-based index $W$ (GADOw et al. 1998) found by Pommerening and GADOW (2000) and Albrecht (2001, p. 87). It is to avoid this problem that the approach proposed in this paper is pointbased.

\section{The angle-based approach}

To describe the assessment of spatial pattern by means of angles, let $D$ be a certain forest stand in which the tree locations are represented by dots. We select $n$ sample points from subregion $D^{*}$ (in order to avoid edge effects, see Fig. 2) using a squared lattice with random starting position and orientation. Consider point $i$ as one of these sample points and let $v_{i j}$ be the vectors joining point $i$ to its $k$ nearest trees, sorted by increasing distance, with $j=1, . ., k$. Let $\alpha_{i j}$ denote the smaller of the two angles (clockwise and counter-clockwise) between the consecutive vectors $v_{i j}$ and $v_{i j+l}$ (with $v_{i k+1}=v_{i l}$ ). Thus $\alpha_{i j}$ is always between $0^{\circ}$ and $180^{\circ}$.

The test for complete spatial randomness (CSR) proposed by ASSUNÇÃO (1994) considers the two nearest trees $(k=2)$ and is based solely on the angle $\alpha_{i 1}$ (Fig. 2a) ${ }^{7}$. The idea is that clustered patterns will yield smaller angles than does CSR, on average. Roughly speaking, this is due to the fact that the probability that a sample point falls outside a cluster increases with increasing clustering. For sample points outside a cluster the angle will tend to be small since it is likely that the two nearest neighbours belong to the same cluster. The opposite is true for simple regular structures. Under CSR the $\alpha_{i 1}$ are uniformly distributed in $\left[0^{\circ}, 180^{\circ}\right]$. To test the null hypothesis that the structure is CSR the Kolmogorov-Smirnov test is applied.

In order to improve the power of test, ASSUNÇÃO and REIS (2000) proposed a modification of the angle test, where the angles between the first and the third, and the first and the fourth, nearest neighbours are also considered. Since all three angles are independently uniformly distributed on $\left[0^{\circ}, 180^{\circ}\right]$ under CSR, this results in a situation equivalent to the original angle test, but the sample becomes three times larger, or two times if only three neighbours are considered. ASSUNÇÃO and REIS (2000) investigated alternative test statistics; the Neyman Smooth Test $p_{2}^{2}$ turned out to be best.

The index $W$ of GADOW et al. (1998) is also based on angles between trees but, instead of using the sample point as origin (to identify the $k$ nearest neighbours and to determine the angles) they shift the origin to the tree that is nearest to the sampling point (tree $a$ ). In other

\footnotetext{
${ }^{6} T$-square sampling (BESAG and GLAVES 1973) tries to avoid the errors caused by those dependencies allowing a search only over a half-plane. This method ensures that the areas searched in the two measures ("nearest tree $a$ to sample point" and "nearest tree to tree $a$ ") cannot overlap, however, it means additional measurements and is, therefore, more time consuming.

${ }^{7}$ Since $\alpha_{i 2}=360^{\circ}-\alpha_{i l}$, it does not give additional information.
} 
words, for the sample point $i$, the $v_{i j}$ are the vectors joining tree $a$ to its $k$ nearest trees, sorted by azimuth in ascending order (with $k=4$, see Fig. 2b). $W$ measures the proportion of angles $\alpha_{i j}$ that are smaller than some specified threshold $\alpha_{0}$, namely the angle between the trees that are arranged in a lattice. Thus $W=1$ is associated with maximum clustering, and $W=0$ with complete regularity. GADOW et al. (1998) defined $\alpha_{0}$ as $90^{\circ}$, but later (HUI and GADOW 2002) changed this into $72^{\circ}$. A practical advantage of the method is that it is unnecessary to measure exact angles, it is only necessary to record whether these are bigger or smaller than $\alpha_{0}$. This can be achieved by using a simple sighting instrument, and even this instrument is only required, if it is doubtful whether an angle falls above or below the threshold. Consequently the measurement effort can be reduced substantially. However, as mentioned before, this way of sampling can lead to biased estimates of the $W$-value in clustered populations ${ }^{8}$.

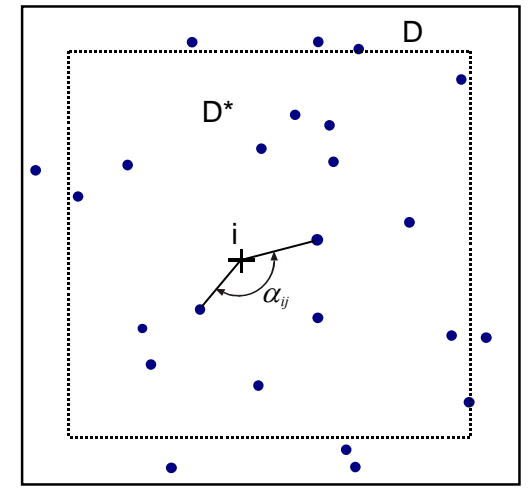

(a)

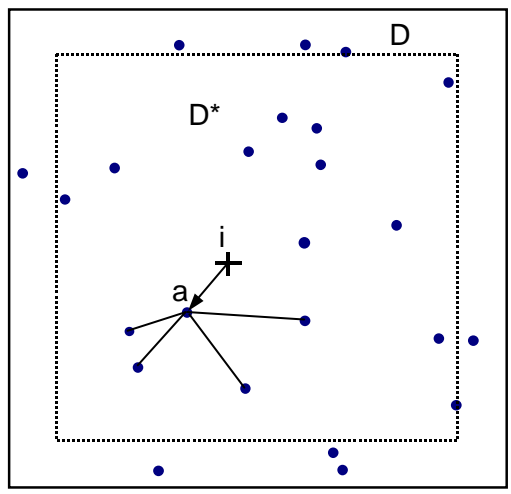

(b)

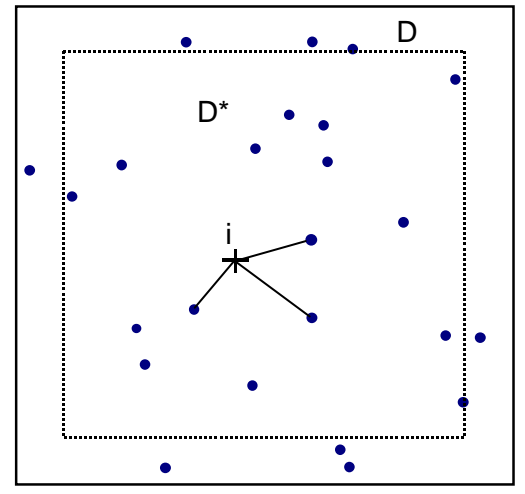

(c)

Fig. 2. The sample unit of 3 types of angle-based measures of spatial pattern at sample point $i$ within a schematic forest stand D: (a) The two nearest trees to point $i$ (angle test by ASSUNÇÃO (1994)), (b) the four nearest trees to tree $a$, where $a$ is the nearest tree to point $i$ (index $W$ by GADOW et al. (1998)), and (c) the three nearest trees to point $i$ (index $W_{P}$ ). All of these indices measure the angle $\alpha_{i j}$ which is the smaller one of the two possible angles formed by the vectors $v_{i j}$ and $v_{i j+1}$ joining point $i(\mathrm{a}, \mathrm{c})$ or tree $a(\mathrm{~b})$ to tree $j$ and tree $j+1$ respectively, with $j=1$ (a), $j=1 . .4$ (b) and $j=1 . .3$ (c), where in (b) and (c) $v_{i j}$ is sorted by azimuth into ascending order. In order to avoid edge effects sample points are chosen from subregion $D^{*}$ with measurements allowed to trees in $D$.

Die Stichprobeneinheit bei 3 Typen von winkelbasierten Indizes für räumliche Verteilungsmuster am Stichprobenpunkt $i$ innerhalb eines schematischen Bestandes $D$ : (a) Die vom Punkt $i$ aus gesehen zwei nächsten Bäume (Winkel-Test nach AsSUNÇÃO (1994)), (b) die vier nächsten Nachbarn zu Baum $a$, wobei $a$ der nächstgelegene Baum zu Punkt $i$ ist (Winkelmaß $W$ nach GADOW et al. (1998)), und (c) die vom Punkt $i$ aus gesehen drei nächsten Bäume (Punktbezogenes Winkelma $\beta W_{P}$ ). Jeder dieser drei Indizes mißt den Winkel $\alpha_{i j}$, d. h. den kleineren der beiden Winkel, die durch die Vektoren $v_{i j}$ und $v_{i j+1}$ gebildet werden, wobei diese den Stichprobenpunkt $i$ (a, c) oder den Baum $a$ (b) mit Baum $j$ bzw. $j+1$ verbinden, mit $j=1$ (a), $j=1 . .4$ (b) und $j=1 . .3$ (c). Ferner ist $v_{i j}$ in (b) und (c) nach dem Azimut aufsteigend sortiert. Um Randeffekte zu verhindern, werden Stichprobenpunkte nur in der Unterfläche $D^{*}$ ausgewählt, wobei alle Bäume in $D$ als Probebäume ausgewählt werden dürfen.

\footnotetext{
${ }^{8}$ HUI and AlBERT (2004) proposed a modified sampling approach which reduces the systematic error significantly but can not eliminate it.
} 
The index presented here, denoted by $W_{P}$, is identical to the index $W$, except that it is based on point-to-tree distances, as proposed by ASSUNÇÃO (1994). It is based on only the three nearest neighbours to the sample point $(k=3$, see Fig. $2 \mathrm{c})$. This choice is mainly motivated by the fact that, as $k$ is increased, it becomes increasingly difficult to confidently identify the $k$-th nearest tree "by eye"; in cases of doubt one has to measure distances, which is the field operation that one is seeking to avoid by using angle-based measures of spatial pattern. Practical tests have shown that the three nearest trees can be determined in most instances without distance measurements. Of course reducing $k$ to 2 would reduce the number of distance measurements even further, but the third tree adds considerable information that compensate for the additional measurement costs, since two trees provide only one evaluable angle, whereas three trees provide three (cf. also ECKMÜLLNER 1998). We define

$$
W_{P, i}=\frac{1}{3} \cdot \sum_{j=1}^{3} z_{i j}, \text { with } z_{j}=\left\{\begin{array}{l}
1, \alpha_{i j}<\alpha_{c} \\
0, \text { otherwise }
\end{array}, \alpha_{c} \leq 120 \text { and } 0 \leq W_{P, i} \leq 1\right.
$$

It follows that $W_{P, i}$ can take on only four different values, namely $0,0.33,0.67$ and $1 . W_{P, i}=0$ indicates regularity, whereas extreme clumping leads to $W_{P, i}=1$ (s. Fig. 3).
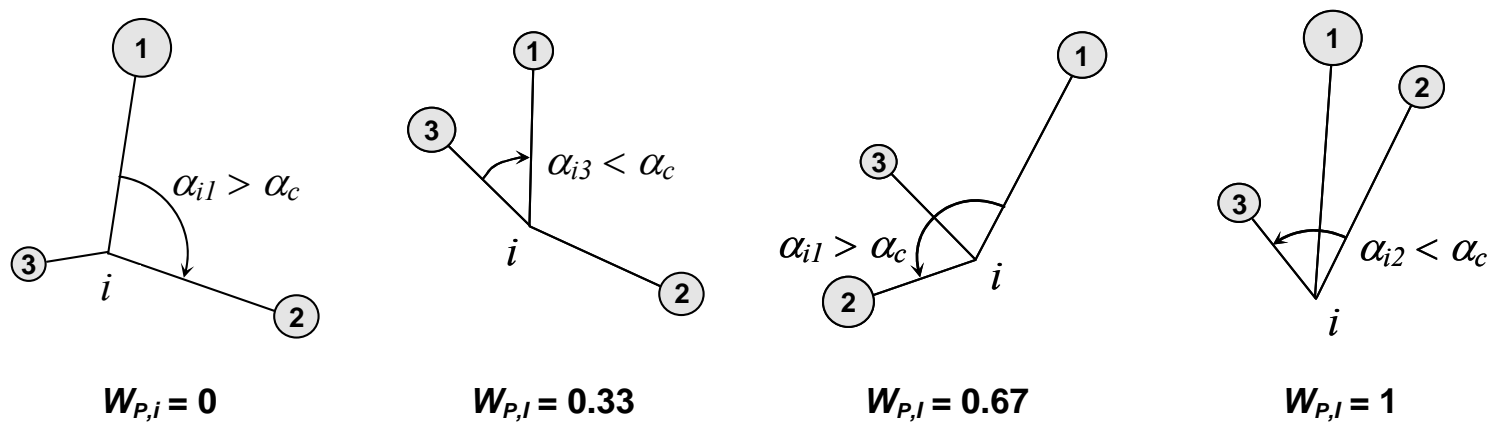

Fig. 3. Possible values of $W_{P}$ at (sample) point $i$. The angles between the trees are denoted by $\alpha_{i j}$, the critical angle by $\alpha_{c}$ which is $90^{\circ}$ in this example. The numbers indicate the order in which the angles are measured. The two right graphs show that there are always two angles between two trees (clockwise and counter-clockwise; a smaller and a bigger one). When calculating $W_{P, i}$ it is always the smaller of the two angles that is used.

Mögliche Werte von $W_{P}$ am (Stichproben-)Punkt $i$. Die Winkel zwischen den Bäumen sind mit $\alpha_{i j}$ bezeichnet, mit $\alpha_{c}$ ist der kritische Winkel angegeben (in diesem Beispiel gleich $90^{\circ}$ ). Die Nummern indizieren die Reihenfolge, in der die Winkel gemessen werden. Die beiden rechten Beispiele zeigen, daß es zwischen zwei Bäumen immer zwei Winkel gibt (im und gegen den Uhrzeigersinn; einen größeren und einen kleineren). Bei der Berechnung von $W_{P, i}$ wird immer der kleinere der beiden Winkel verwendet.

With a systematic sample, as described above,

$$
\hat{W}_{P}=\frac{1}{n} \cdot \sum_{i=1}^{n} W_{P, i}
$$

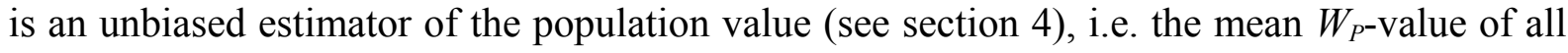
points within a forest stand which build a continuous surface (RIPLEY 1981, p. 19). Since for- 
est inventories are generally based on a systematic sample (e.g. AKÇA 2001, pp. 41), $\hat{W}_{P}$ can be determined with minimum additional effort in the course of such inventories.

The frequency distribution of $W_{P, i}$ under CSR can be derived analytically. For convenience we define $K=3 W_{P, i}$, so that the possible values of $K$ are $0,1,2,3$. It can be shown that for $0^{\circ} \leq \alpha_{c} \leq 90^{\circ}$ :

$$
\begin{aligned}
& P(K=0)=\left(360^{2}-6 \cdot 360 \alpha_{c}+9 \alpha_{c}^{2}\right) / 360^{2} \\
& P(K=1)=\left(6 \cdot 360 \alpha_{c}+5 \alpha_{c}^{2}\right) / 360^{2} \\
& P(K=2)=P(K=3)=3 \alpha_{c}^{2} / 360^{2}
\end{aligned}
$$

One obtains a different set of formulae for the range $\alpha_{c} \in\left(90^{\circ}, 120^{\circ}\right]$, and for $\alpha_{c} \in\left(120^{\circ}, 180^{\circ}\right]$, but these ranges are of less interest than $\alpha_{c} \in\left(0^{\circ}, 90^{\circ}\right]$ and so we won't give the formulae here.

The above result can be used to test the null hypothesis, CSR, against alternatives, by means of a chi-squared goodness-of-fit test, which we will call the $W_{P}$-test. Let

$$
T=\sum_{i=1}^{4} \frac{\left(O_{i}-E_{i}\right)^{2}}{E_{i}},
$$

where $O_{i}$ are the observed frequencies, and $E_{i}$ the expected frequencies under the null hypothesis. For large sample size the distribution of $T$ is approximately $\chi^{2}$ with 3 degrees of freedom. However, the approximation is inaccurate for small sample sizes, specifically if any of the $E_{i}$ is smaller than about 5 . For the sample sizes that are relevant here the minimum expected frequency is much smaller than 5 , and so we need to find a better approximation of the distribution of $T$ in order to obtain accurate critical regions for the test. We approximated the distribution of $T$ by simulating 1 million independent realizations of $T$ under the null hypothesis. As expected, this distribution differs substantially from the $\chi^{2}$-distribution for small sample sizes, but approaches the $\chi^{2}$-distribution as the sample size increases.

The distribution of $T$ under CSR, and hence the power of the test against some alternative, depends on $\alpha_{c}$. In general, however, the value of $\alpha_{c}$ that maximizes the power for a specific alternative hypothesis need not maximize the power against some other alternative. In our application there are two alternative hypotheses of interest, namely "the pattern is more clustered than CSR" and "the pattern is more regular than CSR". It is therefore not surprising to find, as we did, that the value of $\alpha_{c}$ that maximizes the power of the test against the "clustered alternative" differs from that which maximizes the power against the "regular alternative". Thus, in order to achieve maximum power one has to decide, in advance, which alternative is relevant. In practice it is seldom obvious which of these two alternatives is more plausible. To cope with such cases we suggest using an $\alpha_{c}$ which lies between the two optimal values obtained under the above two alternative hypotheses. Thus there are three values of $\alpha_{c}$ that are relevant, one for each of the following alternative hypotheses: 
(a) More clustered than CSR,

(b) more regular than CSR,

(c) either more clustered or more regular than CSR.

In order to obtain the power of test for different $\alpha_{c}$ Monte Carlo simulations of aggregated and regular point processes were carried out. The power of the $W_{P}$-test was compared to that of the modified angle test with three nearest neighbours (ASSUNÇÃO and REIS 2000), using their simulation scheme:

The aggregated patterns were generated according to the modified Thomas process (DigGLE et al. 1976) in $\mathrm{D}=[0,50] \times[0,50]$ and $\mathrm{D}^{*}=[5,45] \times[5,45]$. "Parent points" were generated according to a homogenous spatial Poisson process with $\lambda=0.2$ points per unit area. At each point a Poisson distributed number of trees was generated (with mean $\mu$ ); their positions around the parent point were determined (randomly) in proportion to the density function of a bivariate normal with mean and correlation 0 , and standard deviation $\sigma$. The parameters $\mu$ and $\sigma$ were varied.

Regular patterns were generated according to a Strauss process (STRAUSS 1975) on $\mathrm{D}=[0,70] \times[0,70]$ and $\mathrm{D}^{*}=[10,60] \times[10,60]$. The number of trees was fixed as 200 , and the "radius of inhibition", $r$, was set to 4 . The parameter $\rho$, which measures the "degree of inhibition", was varied from 0.0 to 0.8 (for details, see e.g. ASSUNÇÃO and REIS 2000). In this model $\rho=1$ leads to a Poisson process (CSR); $\rho=0$ produces a pattern where every tree in the region is at least $r$ units from its nearest neighbour.

For each set of parameters we generated 500 realizations of these processes and drew one systematic sample in each case. As did ASSUNÇão and REIS (2000), we used 15 sample points for the regular processes, and 25 for the clustered processes. (Apparently these sample sizes were chosen to keep the sample intensity smaller than $10 \%$ in order to avoid dependencies.) The generated samples were used to compute the power of test for a variety of critical angles, $\alpha_{c}$, ranging from $10^{\circ}$ to $110^{\circ}$, in increments of $5^{\circ}$. The significance level for the chisquared test was set to 0.05 .

Fig. 4 shows the average power against each of the two spatial alternatives to CSR as a function of the critical angle, $\alpha_{c}$. In order to determine the $\alpha_{c}$ which provides the best compromise in the case of an unspecified alternative hypothesis, the values are also shown standardised (by dividing them by their respective maximum value). The value $\alpha_{c}=30^{\circ}$ leads to the greatest power for the clustered alternative which, as expected, is different to the value $\alpha_{c}=70^{\circ}$, which maximizes the power for the regular alternative. A reasonable compromise for the case of an unspecified alternative is the point of intersection of the curves for standardised power, namely $\alpha_{c} \approx 60^{\circ}$. The graph also illustrates that the power is much more sensitive to changes of $\alpha_{c}$ in regular patterns than it is for clustered patterns. 


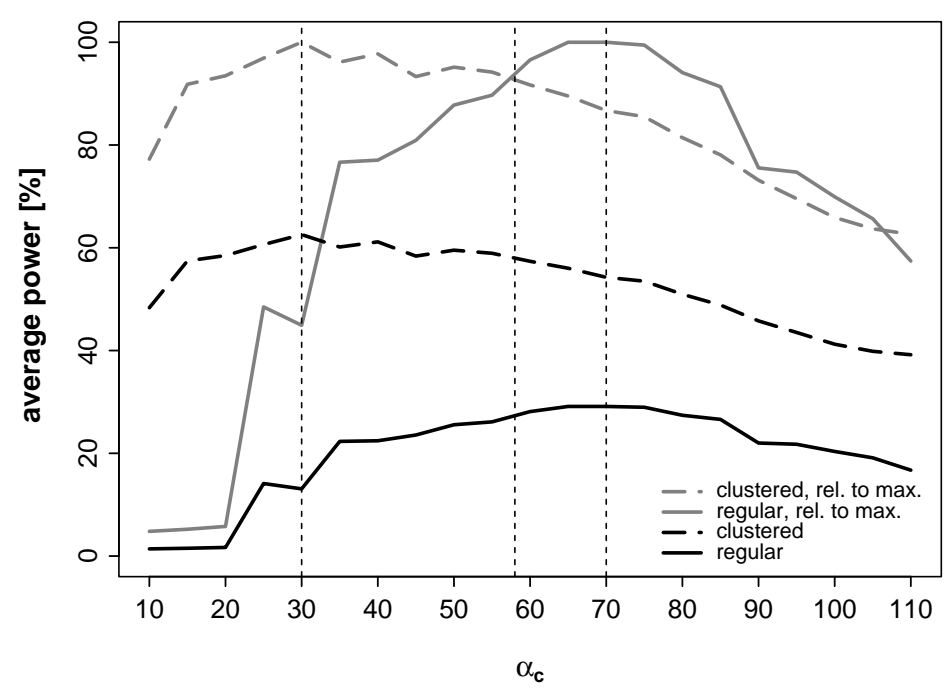

Fig. 4. The average power of the $W_{P}$-test over all simulated clustered and regular patterns, respectively, depending on the critical angle $\alpha_{c}$, absolute and in percent of the maximum value of each of the two pattern type alternatives. For the clustered patterns the maximum power is reached with $\alpha_{c}=30^{\circ}$, for the regular patterns with $\alpha_{c}=70^{\circ}$. If there is no specific alternative hypothesis $\alpha_{c} \approx 60^{\circ}$ is a good compromise.

Stärke des $W_{P}$-Tests im Durchschnitt aller hier simulierten geklumpten bzw. regelmäßigen Verteilungsmuster in Abhängigkeit vom kritischen Winkel $\alpha_{c}$, absolut und in Prozent des jeweils (für jeden der beiden Verteilungstypen) maximalen Wertes. Bei den geklumpten Verteilungen wird die größte Teststärke mit $\alpha_{c}=30^{\circ}$ erreicht, bei den regelmäßigen Verteilungen mit $\alpha_{c}=70^{\circ}$. Wenn keine spezifische Alternativhypothese formuliert wird (werden kann), stellt $\alpha_{c} \approx 60^{\circ}$ einen guten Kompromiß dar.

Fig. 5 illustrates the behaviour of the power in more detail for the above three critical angles. The power decreases with as $\rho$ approaches 1 (Fig 5a), as $\mu$ decreases or $\sigma$ increases (Fig 5b).

(a)

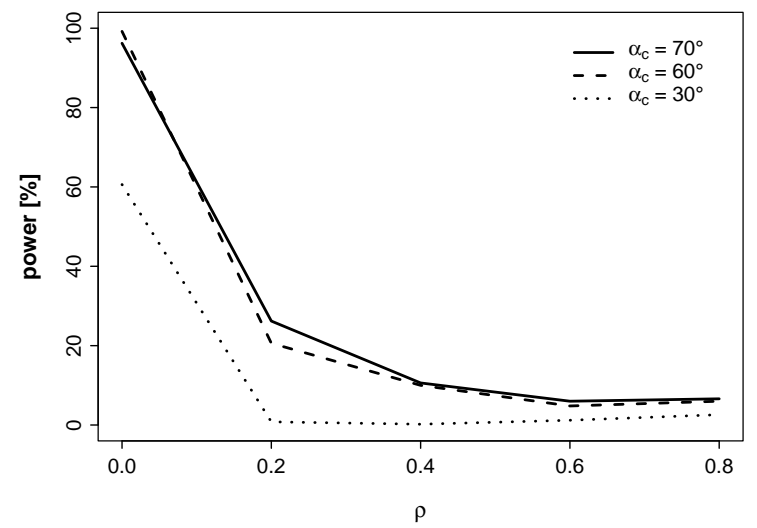

(b)

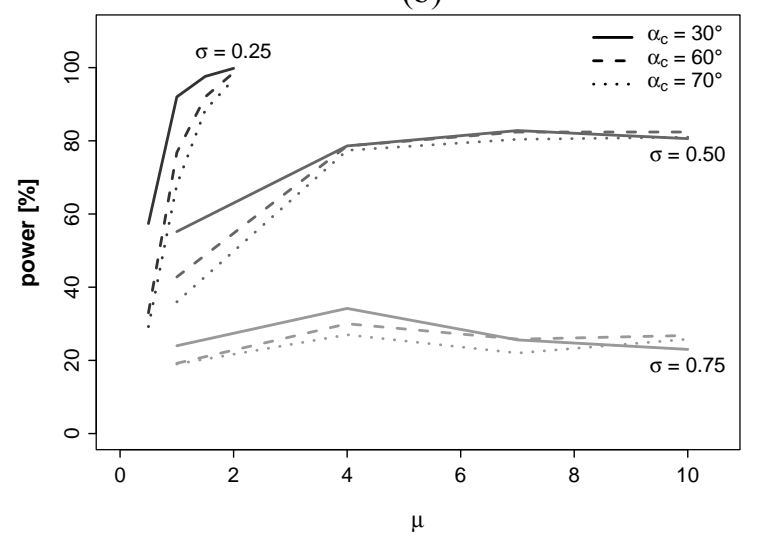

Fig. 5. Tests power against spatial regular (a) and clustered (b) alternatives respectively, depending on type and degree of non-randomness of the investigated patterns $(\rho, \mu$ and $\sigma$ are the parameters of the accordant point processes). The three different line styles denote the tests power obtained with the particular optimum critical angle (solid), the optimum critical angle of the opposed spatial alternative (dotted) and the best critical angle for the case of an unspecified alternative (dashed).

Stärke des $W_{P}$-Tests gegen die Alternativhypothesen „Regelmäßiger als CSR“ (a) und „Geklumpter als CSR“ (b) in Abhängigkeit von Art und Ausprägung der Nichtzufälligkeit der untersuchten Punktmuster ( $\rho, \mu$ und $\sigma$ sind die Parameter der zugehörigen Punktprozesse). Die drei verschiedenen Linientypen zeigen die Teststärken an, die beim jeweils optimalen kritischen Winkel (durchgezogen), beim optimalen kritischen Winkel der entgegengesetzten Alternativhypothese (gepunktet) und beim jeweils besten kritischen Winkel für den Fall der unspezifischen Alternativhypothese (gestrichelt) erreicht werden. 
For clustered patterns the decrease of power that results from using a non-optimal $\alpha_{c}$ is most marked in processes with small and tightly-packed clusters $(\sigma \leq 0.5$ and $\mu<4)$. Although this is not apparent from the figures, the index $W_{P}$ is related to $\rho, \mu, \sigma$, and hence to the power of the test. Moving from a highly regular pattern, through the CSR, and on to a highly clustered pattern, the power, taken as a function of $W_{P}$, decreases, reaches a minimum at CSR, and then increases.

For regular patterns the power decreases rapidly as the degree of regularity decreases. Using the optimal $\alpha_{c}=70^{\circ}$, the power of the $W_{P}$-test is similar to that of the comparable angle test (Version A3N2) of ASSUNÇÃO and REIS (2000), i.e. the test that is also based on the three nearest trees; it is somewhat better for $\rho=0.0$ and only slightly worse for $\rho>0.2$. With clustered patterns the performance of the $W_{P}$-test is clearly worse than that of the angle test if the clusters are very small $(\mu \leq 1)$, especially if a non-optimal $\alpha_{c}$ is used, as would happen if the alternative is unspecified. Apart from that special case, the power is similar. Tab. 1 lists the critical values for $T$ for the three spatial alternative hypotheses and their associated critical angles.

Table 1. Critical values for $\left.T=\sum\left(O_{i}-E_{i}\right)^{2} / E_{i}\right)$ for the three spatial alternative hypotheses and their associated critical angles: "More clustered than CSR" $\left(\alpha_{c}=30^{\circ}\right)$, "More regular than CSR" $\left(\alpha_{c}=70^{\circ}\right)$ and "Either more clustered or more regular than CSR" $\left(\alpha_{c}=60^{\circ}\right)$. The sample size is denoted by $n ; \alpha$ gives the level of significance.

Kritische Werte von $\left.T=\sum\left(O_{i}-E_{i}\right)^{2} / E_{i}\right)$ für die drei Alternativhypothesen zur räumlichen Verteilung und deren zugehörige kritische Winkel: "Geklumpter als bei CSR" $\left(\alpha_{c}=30^{\circ}\right)$, "Regelmäßiger als bei CSR" $\left(\alpha_{c}=70^{\circ}\right)$ und "Entweder geklumpter oder regelmäßiger als bei CSR" $\left(\alpha_{c}=60^{\circ}\right)$. Der Stichprobenumfang ist mit $n$, das Signifikanzniveau mit $\alpha$ angegeben.

\begin{tabular}{crrr}
\hline$n \backslash \alpha$ & 0.1 & 0.05 & 0.01 \\
\hline \multicolumn{4}{c}{$\alpha_{C}=30^{\circ}$} \\
\hline 10 & 6.33 & 7.90 & 18.19 \\
15 & 5.94 & 10.11 & 13.70 \\
20 & 6.96 & 7.94 & 17.42 \\
25 & 5.85 & 8.25 & 14.14 \\
30 & 5.83 & 9.61 & 13.58 \\
Inf & 6.25 & 7.82 & 11.35 \\
\hline \multicolumn{5}{c}{$\alpha_{C}=70^{\circ}$} \\
\hline 10 & 5.51 & 8.55 & 13.12 \\
15 & 5.91 & 7.79 & 12.59 \\
20 & 5.99 & 7.75 & 12.06 \\
25 & 6.12 & 7.69 & 11.77 \\
30 & 5.98 & 7.76 & 11.59 \\
Inf & 6.25 & 7.82 & 11.35 \\
\hline \multicolumn{5}{c}{$\alpha_{C}=60^{\circ}$} & \\
\hline 10 & 6.69 & 7.38 & 13.56 \\
15 & 6.08 & 7.68 & 13.01 \\
20 & 5.89 & 7.88 & 12.70 \\
25 & 6.02 & 7.94 & 12.20 \\
30 & 6.12 & 7.72 & 11.83 \\
Inf & 6.25 & 7.82 & 11.35 \\
\hline
\end{tabular}


In view of these findings we can conclude that, taking inventory costs into account, the $W_{P^{-}}$ test provides an attractive alternative since it requires no exact angle measurements, and hence less sampling effort.

\section{Estimating $W_{P}$ for a stand}

Even when the positions of all trees in the stand are available, calculating the true value of the point-based index $W_{P}$ is not as straightforward as determining that for the tree-based Winkelmass $W$. The problem is that one needs to integrate $W_{P, i}$ over every point in the stand, and there are infinite number of points. However, the "true" $W_{P}$ for the stand can be approximated by subdividing the forest area into a finite number of grid cells (using a lattice), determining $W_{P, i}$ for each cell, and averaging. Fig. 6 illustrates this procedure.

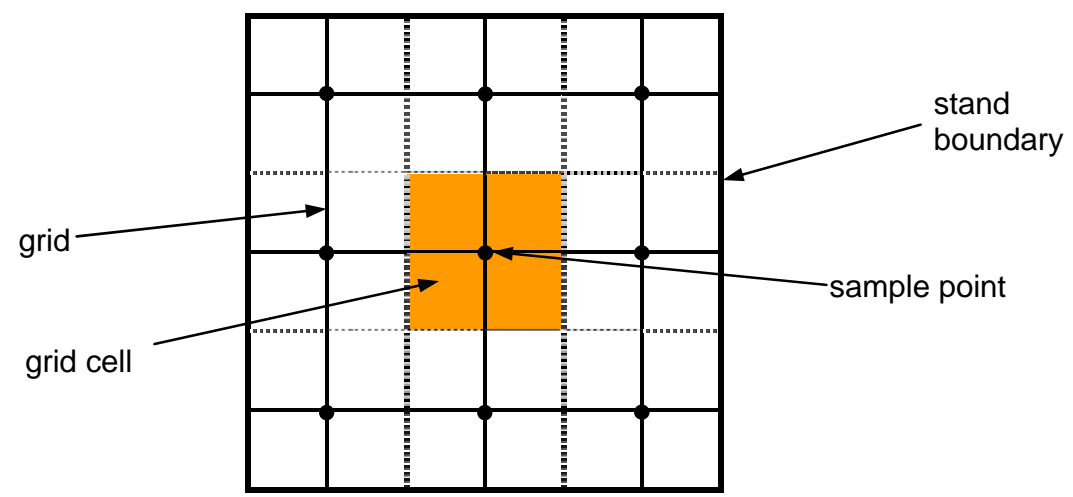

Fig. 6. The aligned centric systematic sample. The orientation of the grid is always parallel to the axes of the coordinate system. This method ensures that the grid cells are always evenly distributed over the stand area which is especially important when the stand shape is symmetric, e.g. rectangular.

Die ausgerichtete und zentrierte systematische Stichprobe. Das Gitternetz ist dabei immer parallel zu den Achsen des Koordinatensystems ausgerichtet, wodurch sichergestellt wird, daß die Gitternetzzellen immer gleichmäßig über die Bestandesfläche verteilt werden, was besonders dann wichtig ist, wenn die Flächenform symmetrisch, $\mathrm{z}$. B. rechteckig ist

The grid cells may be represented as pixels with specific colourings and shadings which code the $W_{P, i}$-value. In this way "cluster maps" can be developed which depict the spatial distribution of the $W_{P}$-classes in a forest stand (see, e.g., Fig. 7). The parameter value of $W_{P}$ is approximated by the arithmetic mean of the pixel values (Eq. 2) and is denoted by $\bar{W}_{P}$.

The accuracy of $\bar{W}_{P}$ improves with decreasing pixel size, and it can be expected to change only slightly below a certain pixel size. The finer the grid-granulation, i.e. the greater the resolution, the closer is the approximation to $W_{P}$, but the computing effort increases quadratically. Thus the resolution should be large enough to achieve satisfactory precision, but not so large as to require excessive computational effort. In determining an appropriate resolution it is necessary to take stand density into account, because it seems reasonable to assume that, for a given spatial pattern and grid size, the precision depends chiefly on the stand den- 
sity; the higher the density the higher the required resolution. Thus it is inappropriate to define an absolute pixel size, but rather to use a range of fractions of the average area occupied by a tree, which we will denote by $F S=$ stand polygon area / number of trees in polygon.
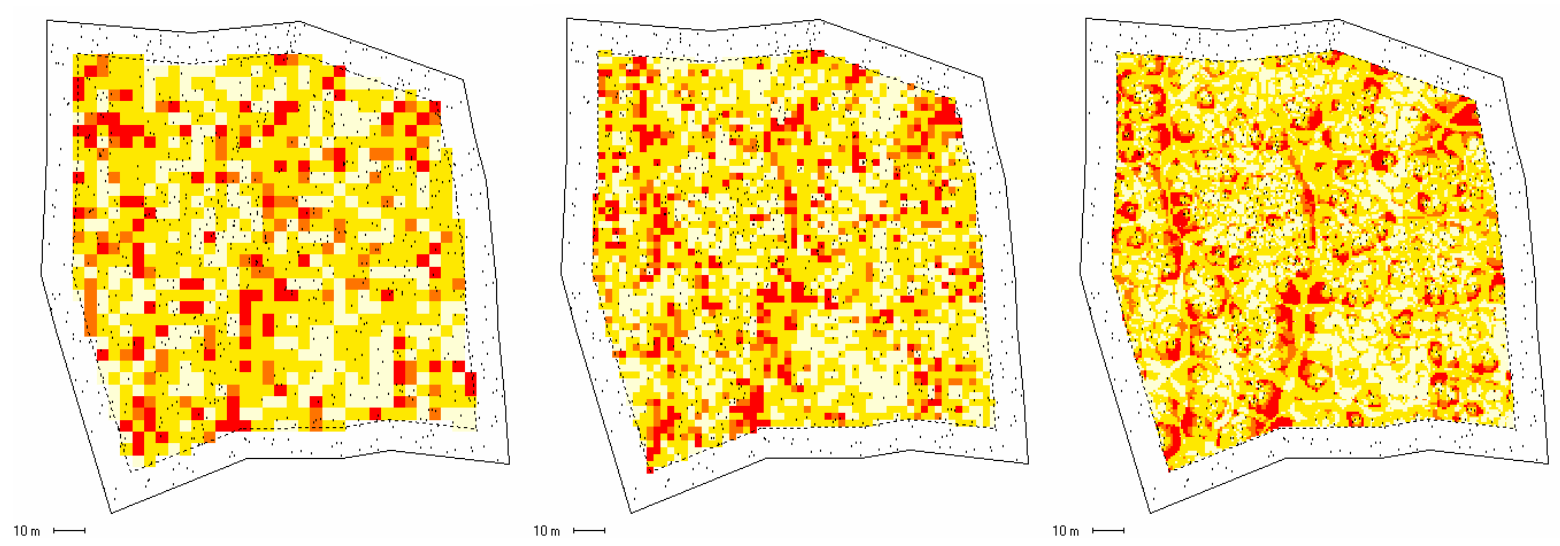

Fig. 7. Distribution of $W_{P, i}$-values in a Swiss Plenterforest using $\alpha_{c}=60^{\circ}$ and pixel sizes of 15,5 and $0.5 \mathrm{~m}^{2}$ (from left to right). The points represent the tree positions. The darker the pixel, the more pronounced is the clustering (and hence the greater is the $W_{P, i}$-value). Of course, the precision of $\bar{W}_{P}$ increases with decreasing pixel size.

Verteilung der $W_{P, i}$-Werte in einem schweizerischen Plenterwald bei einem kritischen Winkel $\alpha_{c}=60^{\circ}$ und einer Pixelgröße von 15, 5 and $0,5 \mathrm{~m}^{2}$ (von links nach rechts). Die Punkte repräsentieren die Baumpositionen. Je dunkler die Pixel, desto ausgeprägter ist die Klumpung (und desto größer der $W_{P, i}$-Wert). Natürlich erhöht sich die Genauigkeit von $\bar{W}_{P}$ mit abnehmender Pixelgröße.

The relationship between grid size and $\bar{W}_{P}$ (with $\alpha_{c}=60^{\circ}$ ) was evaluated using three artificially generated forest stands with different spatial distributions (regular, random, clustered; see Fig. 8). The regular pattern was generated using a Strauss process (STRAUSS 1975) with radius of inhibition $=4$ and $\rho=0$. The aggregated patterns were generated according to the modified Thomas process (DIGGLE et al., 1976) with $\mu=2$ and $\sigma=0.25$. Extreme distributions were generated for demonstration purposes; they allow more general interpretations of the results. To avoid complications caused by edge effects 10 -meter buffer zones were created along the stand boundaries.

Fig. 8 shows the results of the grid analysis with pixel sizes ranging between $1 \%$ and $100 \%$ of $F S$, the mean tree area. For all patterns considered the fluctuations in $\bar{W}_{P}$-values are quite small (less than 0.02) even for pixel size as large as FS. However, problems can be expected in regular and clustered patterns, if the grid is in phase or in counter-phase with the characteristic frequency of the cluster map. The resolution $5 \%$ of $F S$ should ensure reasonable precision in non-degenerate cases.

In what follows we will no longer distinguish between $\bar{W}_{P}$ and $W_{P}$. 
regular
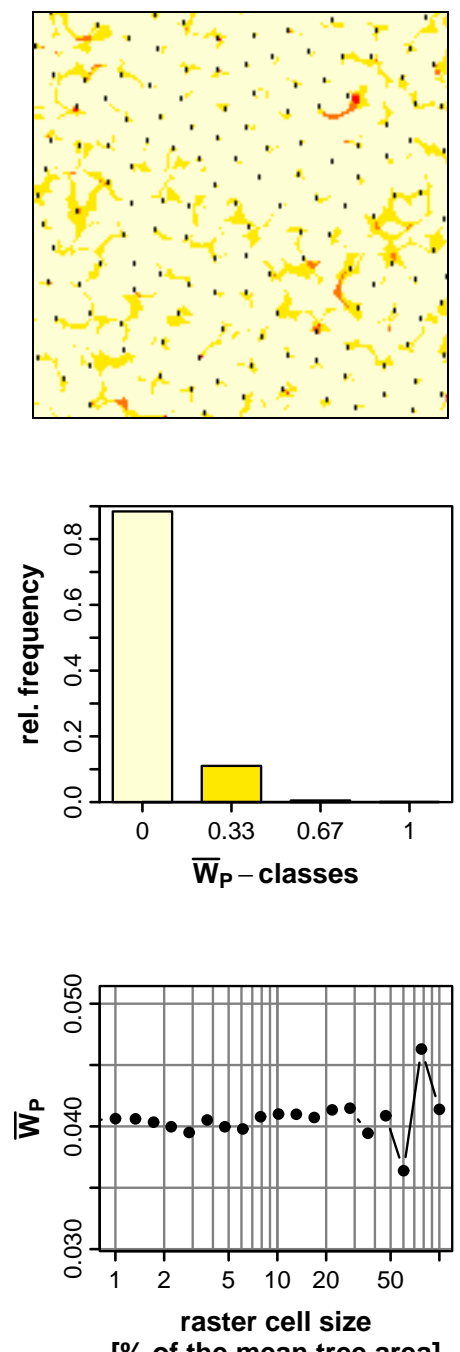

[\% of the mean tree area] random

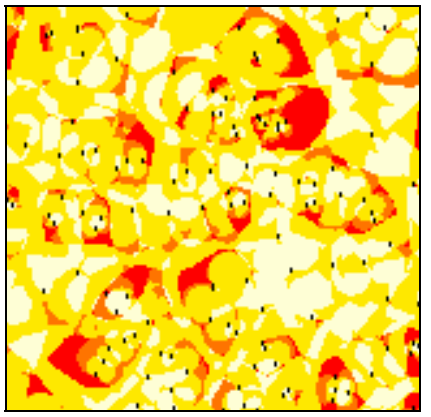

(a)

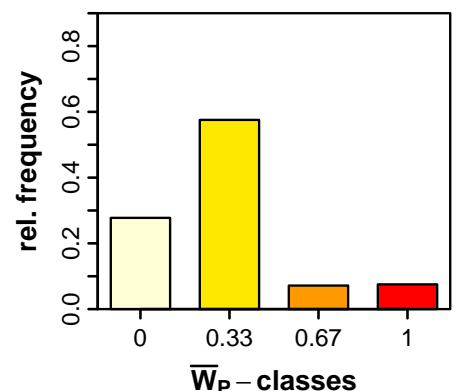

(b)

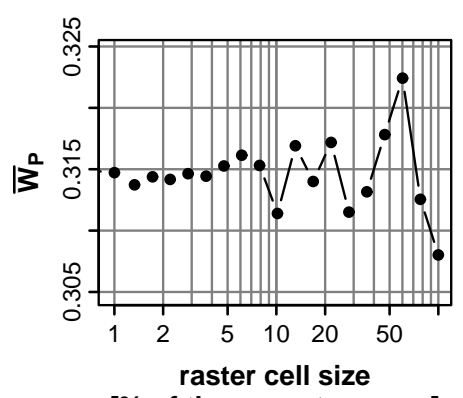

[\% of the mean tree area]
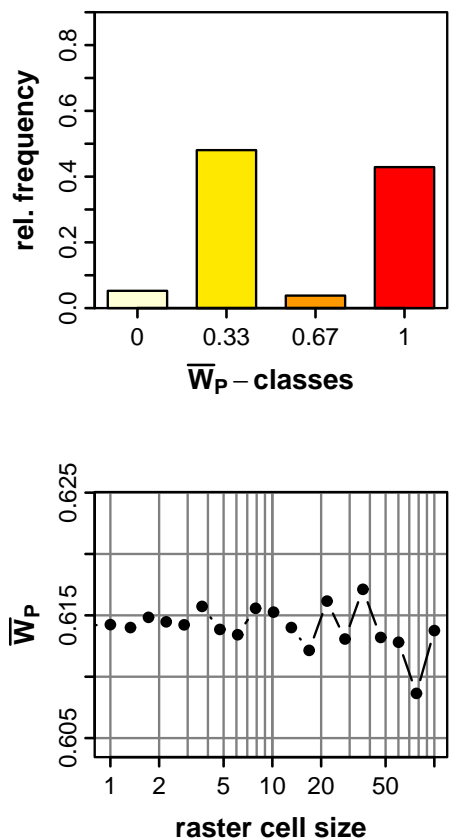

[\% of the mean tree area]

(c)

Fig. 8. Results of the grid analysis for three artificially generated tree populations, each covering an area of 1 ha and comprising approximately 1000 trees: (a) Clippings from the "cluster maps" with superimposed tree positions for pixel size $1 \%$ FS. (b) The associated relative frequencies (as proportions of stand area) of the $W_{P}$-classes. (c) $\bar{W}_{P}$-values for different pixel sizes. The critical angle $\alpha_{c}$ was $60^{\circ}$.

Ergebnis der Rasteranalyse für drei künstlich erzeugte Bestände, jeder 1 ha groß mit ungefähr 1000 Bäumen: (a) Ausschnitte der „Klumpungskarte“ mit überlagerten Baumpositionen bei einer Pixelgröße von $1 \%$ FS. (b) Die zugehörigen relativen Häufigkeiten (Anteile an der Bestandesfläche) der $W_{P}$-Klassen. (c) $\bar{W}_{P}$-Werte bei verschiedenen Pixelgrößen. Der kritische Winkel $\alpha_{c}$ betrug $60^{\circ}$.

\section{Expected value and Standard error}

Determining $W_{P}$ as described above is of course only possible when all the tree positions are known, a situation which is hardly ever encountered in practice. Therefore, it is necessary to estimate $W_{P}$ by means of sampling. The estimator (2) is expected to be unbiased when the sample points are distributed systematically over the polygon area (though in much lower 
density). However, the sample size in a systematic sample, measured in terms of the number of elements drawn independently, is equal to 1 , which means the standard methods for estimating the standard error ${ }^{9}$ of (2) are not applicable. Thus the standard error has to be determined by simulation (cf. e.g. POMMERENING and LEWANDOWSKI 1997; POMMERENING and GADOW 2000).

The expected value, $\mathrm{E}\left(\hat{W}_{P}\right)$, is the average of an infinite number of $\hat{W}_{P}$ realizations obtained by repeatedly randomizing orientations of the sampling grid:

$$
E\left(\hat{W}_{P}\right)=\frac{1}{m} \cdot \sum_{r=1}^{m} \hat{W}_{P, r} \quad \text { for } m \rightarrow \infty
$$

where $\hat{W}_{P, r}$ is the estimate of $W_{P}$ in the $r$-th simulation. The estimator is unbiased if $E\left(\hat{W}_{P}\right)=W_{P}$. The simulation runs can also be used to approximate the standard error:

$$
S E\left(\hat{W}_{P}\right)=\sqrt{E\left(\hat{W}_{P, r}-E\left(\hat{W}_{P}\right)\right)^{2}} \quad \text { with } r=1 . . m \text { and } m \rightarrow \infty
$$

Various methods have been proposed for estimating the standard error of one observation (e. g. CUNIA 1984, pp. 323; WOLTER 1985), however, the validity of such approximations can only be confirmed when additional information about the population is available. For this reason, the formula for a random sample is generally used (AKÇA 2001, pp. 114). This formula gives a good approximation for populations with a random structure, otherwise the real standard error is often overestimated (SABOROWSKI 1992). In order to evaluate the accuracy of the error estimate based on the formula for random samples, its expected value for each stand and sample size can be derived using Eq. 5:

$$
E\left(\hat{S} E\left(\hat{W}_{P}\right)\right)=\frac{1}{m} \sum_{r=1}^{m} \frac{s d\left(\hat{W}_{P, r}\right)}{\sqrt{n_{r}}} \text { for } m \rightarrow \infty
$$

where $s d\left(\hat{W}_{P, r}\right)$ and $n_{r}$ denominate the standard deviation of $W_{P, i}$-values and the sample size in the $r$-th simulation run, respectively.

To obtain the estimates for the values in Eq. 3 - 5 samples of size 5, 10, 20, 30, 40 and 50 were drawn in the three stands shown in Fig. 8. Since the number of simulation runs is limited the simulation average itself is a random variable subject to error. However, KLEINN (1991, pp. 8) showed that the error is negligible when 1000 replications are used, so we used $m=1000$ for each sample size. Tab. 2 gives the design of the simulation experiment.

\footnotetext{
${ }^{9}$ The standard deviation of the estimator, and as the estimator here is unbiased this is also equal to the root mean squared error of the estimator.
} 
Table 2. Statistics of $W_{P}$ calculated by means of sampling simulations in three artificially generated stands with a regular, random and clustered spatial pattern (see Fig. 8). Samples of size 5, 10, 20, 30, 40 and 50 were simulated, with 1000 replications each. The critical angle $\alpha_{c}$ was $60^{\circ}$.

$W_{P}$-Statistiken, berechnet anhand von Stichprobensimulationen in drei künstlich erzeugten Beständen mit regelmäßigem, zufälligem und geklumptem räumlichen Verteilungsmuster (s. Fig. 8). Es wurden Stichpro-ben mit 5, 10, 20, 30, 40 und 50 Probepunkten und jeweils 1000 Wiederholungen simuliert. Der kritische Winkel $\alpha_{c}$ betrug $60^{\circ}$.

\begin{tabular}{ccc}
\hline & Mean & Standard error \\
\hline True value & Grid analysis with $5 \%$ of $F S$ & Equation (4) \\
Estimate & Equation (3) & Equation (5) \\
\hline
\end{tabular}

Fig. 9 shows the simulation results. As expected, the estimator $\hat{W}_{P}$ is virtually unbiased for the entire range of sample sizes and for all three spatial patterns investigated here, and the standard error increases from regular to clustered pattern and with decreasing sample size. For sample sizes greater than 5 the approximations based on Eq. 5 were found to be very close to the true standard errors, even for the non-random population. With sample size 5 the errors were slightly underestimated and the relative amount of this underestimation increases from clustered to regular pattern. The probability of incorrectly identifying the spatial pattern increases at particular high rate when the sample size falls below 20. At the same time larger sample sizes do not reduce the error much. Thus, taking the simulation results in terms of test of CSR (section 2) into account, 20 to 30 sample points would seem to provide a reasonable compromise between precision and sampling effort.

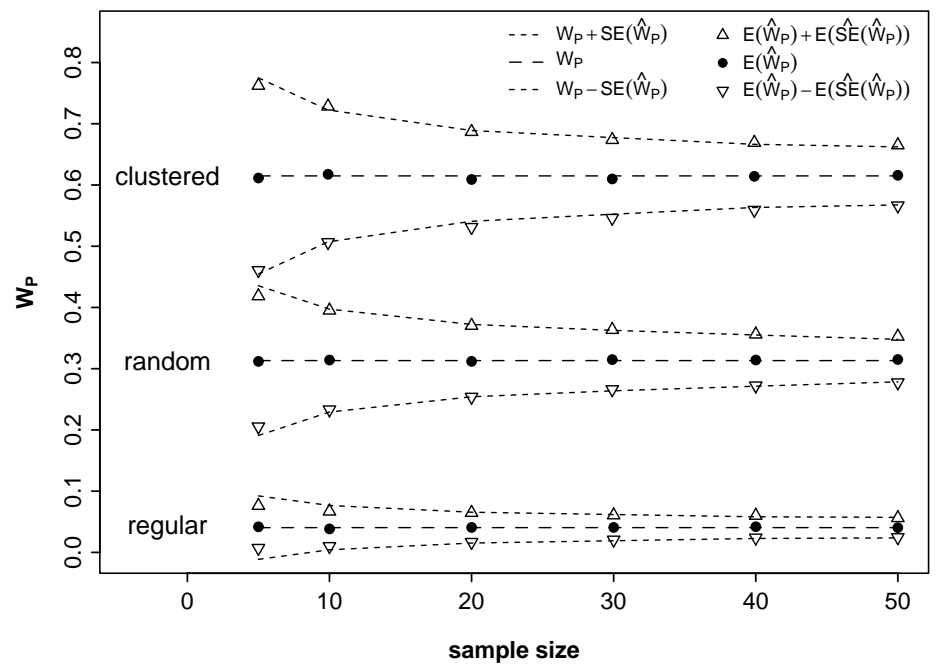

Fig. 9. Expected values and standard errors of $\hat{W}_{P}$ with critical angle $\alpha_{c}=60^{\circ}$ for different sample sizes. The lines show the development of the true values (long dashes = means, short dashes $=$ means $+/$ - standard errors), the symbols indicate the results of the estimates.

Erwartungswerte und Standardfehler von $\hat{W}_{P}$ bei einem kritischen Winkel $\alpha_{c}=60^{\circ}$ für verschiedene Stichprobenumfänge. Die Linien zeigen den Verlauf der wahren Werte (lang gestrichelt = Mittelwerte, kurz gestrichelt = Mittelwerte +/- Standardfehler), währen die Schätzwerte durch die Punktsymbole dargestellt werden. 


\section{Acknowledgments}

The authors wish to acknowledge useful suggestions received from three anonymous referees. Thanks are also due to Prof. Dr. Klaus v. Gadow and Dr. Matthias Albert, Institute of Forest Management, University of Göttingen, and to Dr. Matthias Schmidt, Forest Research Institute Baden-Württemberg, Freiburg i. Br., for helpful comments and discussions. We thank Andreas Zingg, Swiss Federal Research Institute (WSL), Birmensdorf, for permission to use the data from Swiss Plenterforests. This work was funded by the German Research Society DFG (project Ga 473/11-2), which is gratefully acknowledged.

\section{Literature}

AKÇA, A., 2001: Waldinventur. J. D. Sauerländer’s Verlag, Frankfurt a. M., 193 S.

AlBreCHT, A., 2001: Strukturanalyse in einigen Buchen-Naturwaldreservaten in Deutschland, Frankreich und den Niederlanden. Masterarb. Fak. Forstwiss. u. Waldökologie, Univ. Göttingen, 149 S.

ASSUNÇÃO, R., 1994: Testing spatial randomness by means of angles. Biometrics 50: 513 - 537.

ASSUnÇÃO, R., and REIS, I.A., 2000: Testing spatial randomness: A modification of the angle test. Brazilian Journal of Probability and Statistics: $71-86$.

Besag, J.E., and Glaves, J.T., 1973: On the detection of spatial pattern in plant communities. Bulletin of the International Statistical Institute 45: 153 - 158.

BIBER, P., 1997: Analyse verschiedener Strukturaspekte von Waldbeständen mit dem Wachstumssimulator SILVA 2. Vortrag anläßlich der Jahrestagung 1997 der Sektion Ertragskunde im Deutschen Verband Forstlicher Forschungsanstalten. Tagungsbericht: $100-120$.

BYTH, K., and RIPLEY, B.D., 1980: On sampling spatial patterns by distance methods. Biometrics 36: 279 - 284.

CLARK, P.J., and EvANS, F.C., 1954: Distance to the nearest neighbour as a measure of spatial relationship in populations. Ecology 35: $445-453$.

Cox, F., 1971: Dichtebestimmung und Strukturanalyse von Pflanzenpopulationen mit Hilfe von Abstandsmessungen. Mitt. der Bundesforschungsanstalt für Forst- und Holzwirtschaft Reinbek Nr. 67, $161 \mathrm{~S}$.

CuniA, T., 1984: Basic Designs or Survey Sampling: Simple, Stratified, Cluster and Systematic Sampling, $2^{\text {nd }}$ Ed. Forestry, Biometry Monograph Series, Monograph No. 3, State Univ. of New York, College of Environmental Science and Forestry.

Diggle, P.J., Besag, J.E., and Glaves, J.T., 1976: Statistical analysis of spatial point patterns by means of distance methods. Biometrics 32: $659-667$.

ECKMÜllneR, O., 1998: Einfluß der Probeflächengröße auf Maßzahlen der Biodiversität. In: PELZ, D.R., and SABOROWSKI, J. (eds.): Bericht der 11. Tagung der Sektion Forstliche Biometrie und Informatik des Deutschen Verbandes Forstlicher Forschungsanstalten in Freiburg i. Br.: $18-24$.

GADOW, K. V., 1999: Waldstruktur und Diversität. Allg. Forst- u. Jagdztg. 170 (7): 117 - 122.

GAdow, K. V., HuI, G.Y. and AlBERT, M., 1998: Das Winkelmass - ein Strukturparameter zur Beschreibung der Individualverteilung in Waldbeständen. Centralbl. für das ges. Forstw. 115: 1 - 10

Gleichmar, W., and Gerold, D., 1998: Indizes zur Charakterisierung der horizontalen Baumverteilung. Forstwiss. Centralbl. 117: $69-80$.

HINES, W.G.S., and HINES, R.J.O., 1979: The Eberhardt index and the detection of nonrandomness of spatial point distributions. Biometrika 66: $73-80$. 
Hopkins, B., and Skellam, J.G., 1954: A new method determining the type of distribution of plant individuals. Annales Bot., London N. S. 18: 213 - 227.

HUI, G.Y., and ALBERT, M., 2004: Stichprobensimulationen zur Schätzung nachbarschaftsbezogener Strukturparameter in Waldbeständen. Allg. Forst- u. Jagdztg. 175 (10): 199 - 209.

HuI, G.Y., and Gadow, K. V., 2002: Das Winkelmaß - Herleitung des optimalen Standardwinkels. Allg. Forstu. Jagdztg. 173 (10): 173 - 177

KIMMINS, J.P., 1997: Forest ecology - a foundation for sustainable management, $2^{\text {nd }}$ Ed., Prentice Hall, Upper Saddle River, NJ, 596 pp.

KleinN, CH., 1991: Der Fehler von Flächenschätzungen mit Punkterastern und linienförmigen Stichproben. Diss. Forstl. Fakultät, Univ. Freiburg

KÖHL, M., and ZINGG, A., 1995: Eignung von Diversitätsindizes bei Langzeituntersuchungen zur Biodiversität in Waldbeständen. Allg. Forst- u. Jagdztg. 167 (4): 76 - 85.

LewANDOWSKI, A., and Gadow, K. V., 1997: Ein heuristischer Ansatz zur Reproduktion von Waldbeständen. Allg. Forst- u. Jagdzeitg. 168 (9): 170 - 174.

MCPFE, 2003: Improved Pan-European indicators for sustainable forest management as adopted by the MCPFE Expert Level Meeting 7-8 October 2002, Vienna, Austria. Vienna, Austria, MCPFE Liaison Unit. Available online at: http://www.mcpfe.org/system/files/u1/publications/pdf/improved_indicators.pdf.

Отто, H.-J., 1994: Waldökologie. Ulmer, Stuttgart, 391 S.

PommerenING, A., 1998: Fortschreibung von Stichprobendaten mit positionsabhängigen Wuchsmodellen. Vortrag anläßlich der Jahrestagung 1998 der Sektion Ertragskunde des Deutschen Verbandes Forstlicher Forschungsanstalten, Tagungsband: $35-51$.

PommerenING, A., 2006: Evaluating structural indices by reversing forest structural analysis. Forest Ecology and Management 224: $266-277$.

Pommerening, A., and Gadow, K. V., 2000: Zu den Möglichkeiten und Grenzen der Strukturerfassung mit Waldinventuren. Forst u. Holz 55 (19): $622-631$.

Pommerening, A., and LewANDOWSKI, A., 1997: Zur Stichprobensimulation in strukturreichen Wäldern. Allg. Forst- u. Jagdztg. 168 (3/4): $63-67$.

Pretzsch, H., 1995: Zum Einfluß des Baumverteilungsmusters auf den Bestandeszuwachs. Allg. Forst- u. Jagdztg. 166: 190 - 201.

PRETZSCH, H., 1997: Analysis and modeling of spatial stand structures. Methological considerations based on mixed beech-larch stands in Lower Saxony. Forest Ecology and Management 97: 237 - 253.

RIPLEY, B.D., 1981: Spatial statistics. Wiley \& Sons, New York, 252 pp.

SABOROwSKI, J., 1992: Ein Diskussionsbeitrag zum Thema Systematische Stichproben in der Waldinventur. Allg. Forst u. Jagdztg. 163 (6): $107-110$.

SCHREUder, H.T., Gregoire, T.G., and Wood, G.B., 1993: Sampling methods for multiresource forest inventory. Wiley \& Sons, New York, 446 pp.

SMALTSCHINSKI, TH., 1998: Charakterisierung von Baumverteilungen. Forstwiss. Centralbl. 117: 355 - 361.

SPELLMANN, H., 1995: Vom strukturarmen zum strukturreichen Wald. Forst u. Holz 50 (2): 35 - 44.

STAUPENDAHL, K. ,1997: Ein neues Stichprobenverfahren zur Erfassung und Beschreibung von Naturverjüngung. In: Pelz, D.R. (eds.): Deutscher Verband Forstlicher Forschungsanstalten, Sektion Forstliche Biometrie und Informatik - 10. Jahrestagung, Freiburg, 24. - 26. September 1997, IUFRO, Grüne Reihe: 32 49.

STAUPENDAHL, K., 2006: Die modifizierte 6-Baum-Stichprobe - ein geeignetes Verfahren zur Erfassung von Waldbeständen (submitted to Allg. Forst- u. Jagdztg.)

STRAUSS, D.J., 1975: A model for clustering. Biometrika 62: 467 - 475.

SZARO, R.C., and JOHnSTON, D.W., 1996: Biodiversity in managed landscapes. Oxford University Press, 778 pp. 
Upton, G., and Fingleton, B., 1989: Spatial Data Analysis by Example. Vol. 2: Categorical and Directional Data. John Wiley \& Sons, Chichester, 416 pp.

Upton, G., and Fingleton, B., 1990: Spatial Data Analysis by Example. Vol. 1: Point Pattern and Quantitative Data. $2^{\text {nd }}$ Ed., John Wiley \& Sons, Chichester, 410 pp.

VANCLAY, J., 1998: Towards more rigorous assessment of biodiversity. In: BACHMANN, P., KÖHL, M., and PÄIVINEN, R. (eds.): Assessment of biodiversity for improved forest planning. Kluwer Academic Publishers: $211-232$.

WOLTER, K.M., 1985: Introduction to variance estimation. Springer, New York, 427 pp. 



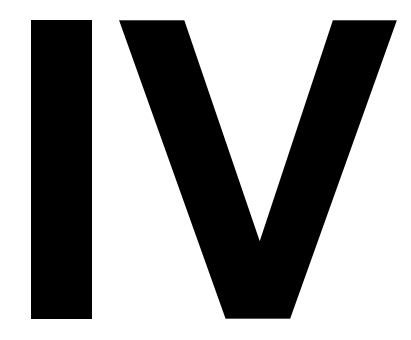





\title{
Modelling thinnings based on the ratio of relative removal rates \\ Modellierung von Durchforstungen auf der Basis relativer Entnahmeraten
}

\author{
KAI STAUPENDAHL \\ University of Joensuu, Finland, Faculty of Forestry Research Notes 97: 183 - 194
}

Schlagwörter: Durchforstung, Durchforstungsmodell, Durchmesserverteilung, Buche.

\section{Zusammenfassung}

Die quantitative Beschreibung von Durchforstungseingriffen ist eine wesentliche Voraussetzung für die Prognose der zukünftigen Bestandesentwicklung. Darüber hinaus können die anfallenden Sortimente nur dann geschätzt werden, wenn die durchforstungsbedingten Änderungen der Durchmesserverteilung vorausgesagt werden können. In dieser Arbeit wird eine Methode vorgestellt, die diese Veränderung der Durchmesserverteilung auf der Basis von drei Variablen modelliert, nämlich dem Durchmesser des Grundflächenmittelstamm und der Stammzahl des Gesamtbestandes und dem Quotient aus den relativen Entnahmeraten der Stammzahl und der Grundfläche, wobei der letzte Parameter die Durchforstungsart beschreibt. Mit Hilfe dieses Modells wird der ausscheidende Bestand geschätzt. Die Durchmesserverteilung des verbleibenden Bestandes ergibt sich dann durch klassenweise Subtraktion der Häufigkeiten des ausscheidenden Bestandes von denen des Gesamtbestandes, die wiederum als bekannt vorausgesetzt werden. Wie anhand unabhängiger empirischer Daten und verschiedener Prüfgrößen gezeigt werden kann, erlaubt ein solches Modell zuverlässige Schätzungen.

Key words: Thinning, thinning model, diameter distribution, beech.

\begin{abstract}
A quantitative description of a thinning operation is an essential prerequisite for forecasting future stand development. Furthermore, the product assortments that will become available can be predicted more precisely, if one is able to project changes caused by thinnings. In this presentation a method is introduced, which models the change of the dbh-distribution of evenaged beech stands on the basis of three variables: The arithmetic mean diameter of the total stand, the total stem number and the quotient of relative removal rates of stem number and basal area, whereas the third model parameter is used to control the type of thinning. The main emphasis is on the estimation of the removed stand. The dbh-distribution of the remain-
\end{abstract}


ing stand can easily be calculated by subtracting the classwise frequencies of the removed stand from those of the total stand, which are regarded as known or are being estimated applying appropriate methods. Independent empirical data can be described by the model with adequate accuracy, as shown by several test criteria.

\section{Introduction}

In modelling thinning operations, the frequency distribution of the tree diameters is of central importance. It describes the horizontal structure of a stand and thus delivers essential information about product yields (WENK 1990, p. 189). A number of models have already been developed to specify the effect of a thinning on a diameter distribution (e. g. KENNEL 1972; Preussner 1974; Alder 1979; RÖMISCH 1983; GAdOw 1987; LEMM 1991; Álvares GONZÁles 1997; GADOW and Hui 1997). Most of these models are based on theoretical distribution functions and estimate the change of the distribution parameters with specific regression equations. A disadvantage of these equations is however that usually only predefined thinning types can be reproduced. Therefore neither the values of the regression parameters, nor the particular form of the regression equations are necessarily applicable in other circumstances. In fact, there is no clear methodology of how such equations should be derived for other data sets (MURRAY and GADOW 1991). Even though a model includes parameters which control the thinning type, these parameters are usually difficult to interpret.

In this investigation the ratio between the removal rates of stem number and basal area was used to describe the thinning type. This ratio proposed by KASSIER (1993) is simple to calculate and easy to interpret. Based on extensive data from long-term thinning trials gathered by the Forestry Research Station of Lower Saxony a thinning model was developed, which primarily estimates the diameter distribution of the removed stand (the trees that are going to be removed). The accuracy of this model was tested by using an independent data set and different test criteria.

\section{Data}

The parameters of the thinning model are based on 67 diameter distributions of even-aged beech stands from 9 long-term thinning trials of the Northwest German Forest Research Station, Göttingen (NW-FVA). The ages of these stands range from 35 to 140 years. The thinnings correspond to high and low thinnings according to the definition of the ASSOCIATION OF FORESTRY RESEARCH STATIONS (1902). Only distributions with at least 5 trees in the removed stand were considered. Since the data of one plot partially contain several observations at different ages, the observations are not independent. Therefore, strictly speaking, significance 
test criteria are not applicable, the results of such tests can however indicate the strength of the correlations.

For validating the thinning model 30 diameter distributions of even-aged beech stands of the State Forestry District Paderborn (North Rhine-Westphalia) were used. At least 200 trees were measured in every stand. The trees that had to be removed were signed before the measurements by the responsible district forester. The age of these stands varies between 50 and 140 years, with a preponderance of the middle age classes. In all stands with more than one tree layer only the upper canopy was considered, since the approach presented here is suitable only for unimodal diameter distributions.

\section{Weight and type of thinnings}

Depending on the type of diameter distribution used (stem frequency or basal area per dbh class), the weight of a thinning can be described by the proportion of the stem number $r N$ or the proportion of the basal area $r G$, which is going to be removed:

$$
\begin{aligned}
& r N= \frac{N_{\text {rem }}}{N_{\text {total }}} \\
& r G= \frac{G_{\text {rem }}}{G_{\text {total }}}, \\
& \text { with } \quad N_{\text {rem }}, N_{\text {total }}=\text { number of stems per ha, removed and before thinning } \\
& G_{\text {rem }}, G_{\text {total }}=\text { basal area per ha, removed and before thinning }
\end{aligned}
$$

Following a proposal by KASSIER (1993), the type of thinning can be described by the ratio of the relative removal rate $r N$ to the relative removal rate $r G$. This ratio is indicated by the symbol $N G$ :

$$
\begin{aligned}
& N G=\frac{r N}{r G} \\
& \text { with } r N, r G=\text { relative removal rates of stem number and basal area respectively }
\end{aligned}
$$

By transforming formula (3) we obtain:

$$
d g_{\text {rem }}=N G^{-0,5} \cdot d g_{\text {total }}
$$

with $d g_{\text {rem }}, d g_{\text {total }}=$ quadratic mean diameter of the removed trees and the stand before thinning 
The quadratic mean diameters of the removed stand and the total stand are identical when $N G=1$. In this case, the thinning is likely to be unselective ${ }^{1}$. If $N G<1$, the proportion of removed stem number is less then the proportion of removed basal area, that is, the quadratic mean diameter of the removed stand is bigger than the quadratic mean diameter of the stand before thinning. Consequently, a high thinning can be assumed. Vice versa $N G$ values $>1$ correspond to a low thinning. At the same time the respective character of the thinning type is the more distinct the more the $N G$ value differs from 1 .

However, due to diverse stand structures and subjective interpretation of thinning rules, in real stands the $N G$ values vary for the same type of thinning. Fig. 1 shows the variation of $N G$ values for high and low thinnings in the 67 stands studied here. Nevertheless, the difference of the means is highly significant ( $\alpha=0.01$ ). Fig. 1 even shows that the border between high and low thinnings does not correspond to $N G=1$.

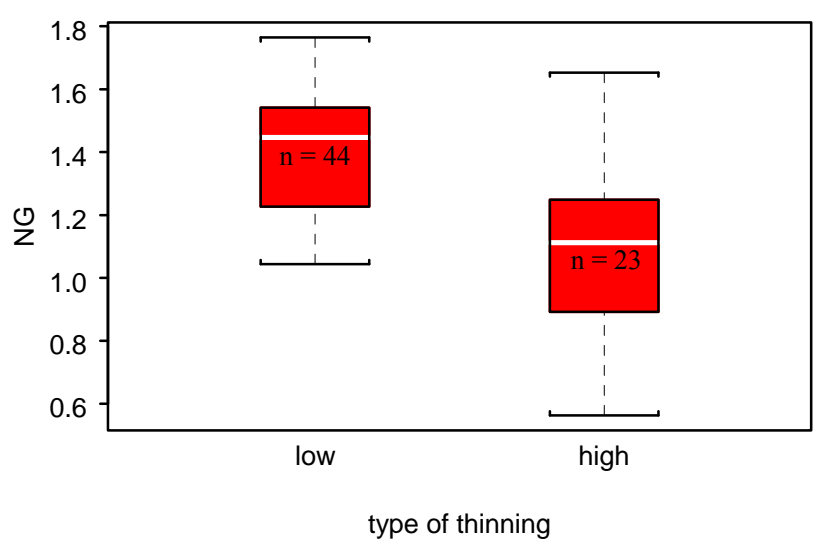

Fig. 1. Box-Whisker-Plots of the $N G$ values calculated for high and low thinnings in 67 even-aged beech stands. The plots are showing the median, the central $50 \%$ and the range of the $N G$ values.

\section{Thinning model}

In order to asses the change in the diameter distribution caused by thinning operations, a thinning model was developed, that estimates the diameter distribution of the removed stand using the Weibull density function (cp. STAUPENDAHL and PUUMALAINEN 1999). The threeparametric version of this function has the following form:

$$
\begin{aligned}
& f(x)= \frac{c}{b} \cdot\left(\frac{x-a}{b}\right)^{c-1} \cdot e^{-\left(\frac{x-a}{b}\right)^{c}} \quad(a \leq x<\infty) \\
& \text { with } \quad \begin{aligned}
x & =\text { continuous random variable }(\text { e. g. DBH }) \\
a & =\text { location parameter, representing the lower bound } \\
b & =\text { scale parameter } \\
c & =\text { shape parameter }
\end{aligned}
\end{aligned}
$$

\footnotetext{
${ }^{1}$ A special case would be the removal of only very thin and very thick trees.
} 
If the function parameters are known, the classwise frequencies of the appropriate diameter distribution can be calculated as follows:

$n_{i}=k \cdot f\left(d_{i}\right) \cdot N$

with $n_{i}=$ absolut frequency in the $\mathrm{i}$-th diameter class

$d_{i}=$ mean of the $\mathrm{i}$-th diameter class

$k=$ class width

$N=$ total stem number

The objective was to find appropriate estimating functions for the expected Weibull parameters with a given stand and thinning type. To obtain such equations, the following method was used:

At first the Weibull function was fitted to each of the 67 empirical diameter distributions of the total, removed and remaining stands, using the method of moments as proposed by GARCIA (1981). A problem when fitting the Weibull function concerns the correlation between the three function parameters. This means that different combinations of parameter values can result in very similar distributions. Particularly, the location parameter $a$, which represents the lower bound of the distribution, has a strong effect on both the scale and shape parameter. Naturally, this complicates the parameter estimation. Therefore, in this investigation the $a$ parameter of the removed stand was ,fixed" by using the same value as that estimated for the total stand ${ }^{2}$. This results in a more directional reaction of the scale and shape parameter on different thinning types.

The second step involves a regression analysis with the Weibull parameter as response variable and the quadratic mean diameter of the total stand, the stem number of the total stand and the $N G$-ratio as predictor variables ${ }^{3}$. This results in the following equations $\left(R^{2}\right.$ means the coefficient of determination):

$$
\begin{array}{rlr}
a_{\text {rem }}=a_{\text {total }} & \\
b_{\text {rem }}=0.1230+1.0670 \cdot\left(d g_{\text {rem }}-a_{\text {rem }}\right) & R^{2}=0.99 \\
c_{\text {rem }}=8.0665+0.0554 \cdot d g_{\text {rem }}-2.2392 \cdot \ln \left(a_{\text {rem }}\right)-0.0013 \cdot N_{\text {total }} & R^{2}=0.39 \\
\text { with } \quad a_{\text {rem }}, b_{\text {rem }}, c_{\text {rem }} & =\text { Weibull parameter of the removed stand } & \\
a_{\text {total }} & =\text { Weibull parameter } a \text { of the stand before thinning } & \\
d g_{\text {rem }} & =\text { quadratic mean diameter of the removed stand } \\
N_{\text {total }} & =\text { stem number of the stand before thinning } &
\end{array}
$$

\footnotetext{
${ }^{2}$ This proceeding is feasible, because every thinning can start with the smallest diameter.

${ }^{3}$ A preliminary data analysis had shown that other stand variables expected to be known improve the accuracy of the estimates only very little.
} 
If the parameter $a$ of the total stand is unknown, it can be estimated by the lower bound diameter. The form of the estimation function for the parameter $b$ of the removed stand based on an investigation of GADOW (1987, p. 100), who found an approximately functional relationship between the parameter $b$ and the difference between the arithmetic mean diameter $\bar{d}$ and the parameter $a$ in plantations of Pinus patula. The coefficients and the high $R^{2}$ of the estimation function for $b_{\text {rem }}$ validate the presumption that there is a simple relationship $b=\bar{d}-a$. The $d g$ of the removed stand, which is needed for the estimation of the scale and shape parameter are obtained from formula (4), which means that - as intended - different thinning types produce different distribution parameters.

Thus, for modelling the thinning effect on a given diameter distribution we need the values of the following variables:

1.) The $d g$, the Weibull parameter $a$ and the stem number of the total stand.

2.) Any combination of two of the three variables $r N, r G$ and $N G$, to control the weight and type of thinning. The value of the third variable is determined by the other ones (see formula (3)).

If the distribution of the removed stand is estimated, the distribution of the remaining stand can easily be calculated by subtracting the class wise frequencies of the removed stand from those of the total stand. In order to get "smoothed" distributions the fitted Weibull distributions of the total stand instead of the empirical distributions were used.

With this thinning model the diameter distributions of the removed and remaining stand were estimated for the independent data set of 30 even-aged beech stands. For validating the model, both for the removed and the remaining diameter distributions, four different criteria were calculated. The criteria 1 and 2 refer to the error of the relative stem number distributions, whereas the criteria 3 and 4 measure the error of the absolute basal area distributions:

1.) Maximal absolute difference $K S$ between the empirical and estimated cumulative distribution function (Kolmogorov-Smirnov-Test).

2.) Relative Discrepancy $r D$ (cf. GREGORIUS 1974):

$$
\begin{gathered}
r D=\frac{1}{2} \cdot \sum_{i=1}^{n}\left|\hat{H}_{i}-H_{i}\right| \\
\text { with } \begin{aligned}
H_{i} & =\text { empirical relative frequency of the } \mathrm{i} \text {-th diameter class } \\
\hat{H}_{i} & =\text { estimated relative frequency of the } \mathrm{i} \text {-th diameter class } \\
n & =\text { number of diameter classes }
\end{aligned}
\end{gathered}
$$

This criterion measures the relative proportion, which had to be interchanged between the classes to obtain the true distribution from the estimated one. Accordingly $1-r D$ is the 
proportion common to both distributions. The value 1 means the distributions are totally different, whereas they are identical, if $r D=0$.

3.) Relative error of the quadratic mean diameter $d g$ :

$$
\begin{aligned}
& \text { re.dg }=\frac{\hat{d} g-d g}{d g} \\
& \text { with } \quad \begin{array}{l}
d g=d g \text { of the empirical diameter distribution } \\
\hat{d} g=d g \text { of the estimated diameter distribution }
\end{array}
\end{aligned}
$$

4.) Relative error of the basal area $\mathrm{G}$ :

$$
\begin{aligned}
& r e . G=\frac{\hat{G}-G}{G} \\
& \text { with } \begin{array}{l}
G=\text { basal area of the empirical diameter distribution } \\
\hat{G}=\text { basal area of the estimated diameter distribution }
\end{array}
\end{aligned}
$$

\section{Model validation}

The results of the validation are shown in Fig. 2. There are clear differences between the error values of the removed and remaining diameter distributions: The means and - with one exception - even the variation of the test criteria are obviously higher for the removed stand than for the remaining stand. Nevertheless, if one compares the $K S$ values to the critical boundaries of the Kolmogorov-Smirnov-Test for two samples with an error probability of $5 \%$, in all cases the estimated and the true distribution originate from the same population.
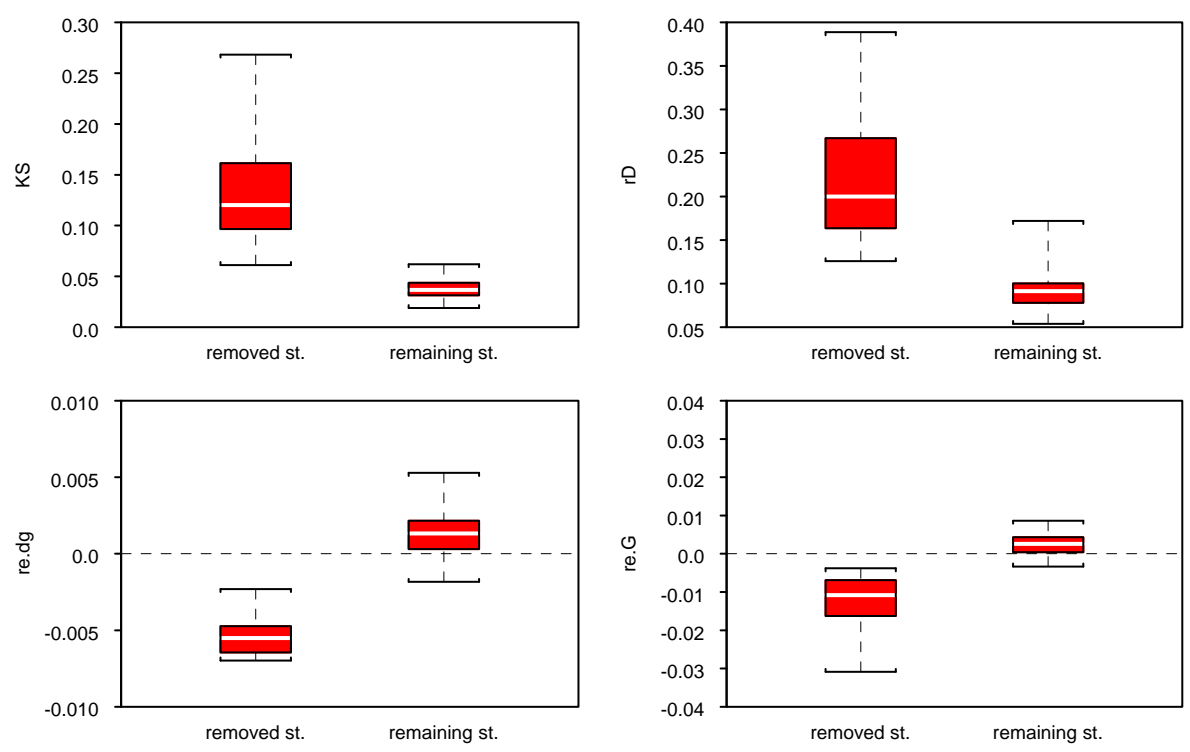

Fig. 2. Box-Whisker-Plots showing the error means and variations of the estimated diameter distribution. The median, the central $50 \%$ and the range of the KS values, the relative Discrepancy $r D$ and the relative errors of quadratic mean diameter re.dg and basal area re. $G$ were calculated for both the diameter distributions of the removed and the remaining stand. 
Basically, there are two reasons for a discrepancy between the estimated and true diameter distribution, which can be quite high in the removed stands: Either a divergence of the Weibull parameters or a high variation of class frequencies in the true distribution. A divergence of the distribution parameters is caused by the thinning model, whereas the variation of class frequencies depends on the type of stand and on the number of trees measured. Probably this is the reason for the higher error values of the removed stand, since they do not contain as many trees as the remaining stand. The error of the quadratic mean diameter is diminutive and the same applies - more or less - to the basal area error. In both cases there is a slight underestimation in the removed stand and, consequently, an overestimation in the remaining stand, which is admittedly lower than the underestimation due to the calculation of relative errors. However for practical purposes this bias is not serious. Fig. 3 shows an example of an estimated diameter distribution, both for high and low thinning.
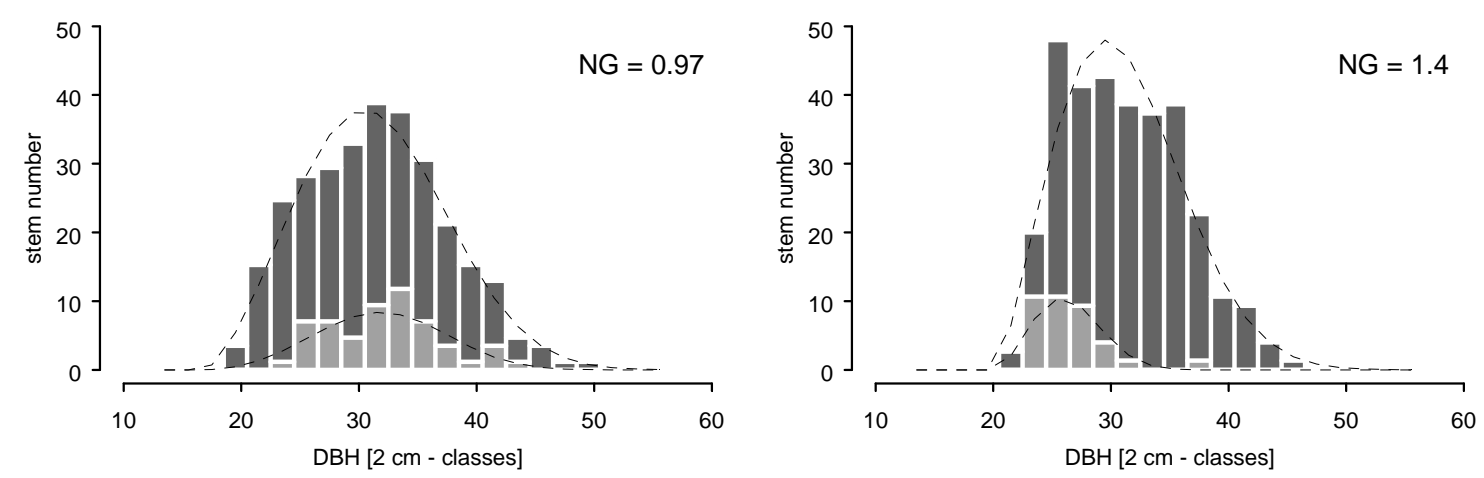

Fig. 3. True and estimated diameter distributions of two compartments from Paderborn with different thinning regimes. As indicated by the $N G$ values the left stand was high thinned, whereas the right stand was low thinned. The bars represent the absolute empirical frequencies (dark grey: remaining stand, light grey: removed stand). The estimated distributions are indicated by the dotted lines.

\section{Conclusions}

Using the thinning model presented here, the diameter distributions of the removed and remaining trees in even-aged beech stands can be estimated with adequate accuracy. The method seems to be suitable even for other tree species, as long as they have unimodal diameter distributions. However, in addition to the errors mentioned here, other errors may occur, if the estimated distribution of the total stand is inaccurate or the real thinning type and weight does not correspond to the one used in the thinning model.

An advantage of this approach is the compatibility with management models, which prescribe a certain reduction of stem number and basal area in a given stand age. In this context the $N G$ ratio can serve as an interface between the thinning model and these management models. It would be interesting to test the approach in a modelling system for plantations of pine or eucalyptus. 


\section{Acknowledgments}

The Author thank Prof. Dr. Hermann Spellmann and Prof. Dr. Jürgen Nagel, Northwest German Forest Research Station (NW-FVA), Göttingen, for the kindly permission to use comprehensive datasets from thinning trials in beech and spruce stands. This work was funded by the German Research Society DFG (project Ga 473/11-1), which is gratefully acknowledged.

\section{Literature}

Alder, D., 1979: A Distance-Independent Tree Model for Exotic Conifer Plantations in East Africa. For. Sci. 25: 59 - 71 .

Álvarez Gonzales, J.G., 1997: Análisis y caracterización de las distribuciones diamétricas de Pinus pinster Ait. en Galicia. Diss. Univ. Madrid.

GADOW, K. V., 1987: Untersuchungen zur Konstruktion von Wuchsmodellen für schnellwüchsige Plantagenbaumarten. Forstliche Forschungsberichte München. Bd. 77. 147 S.

GADOw, K. v. und HuI, G. Y., 1997: Modellierung forstlicher Eingriffe in Plantagenwäldern. Forstarchiv 68: 59 $-63$.

GARCIA, O., 1981: Simplified method-of-moments estimation for the weibull distribution. New Zealand Jour. For. Sci. (11) 3: 304 - 305.

Gregorius, H.-R., 1974: Genetischer Abstand zwischen Populationen - Zur Konzeption der genetischen Abstandsmessung. Silvae Genetica 23: 22-27.

KASSIER, H.W., 1993: Dynamics of diameter and height distributions in commercial timber plantations. $\mathrm{PhD}$ dissertation, Faculty of Forestry, Univ. of Stellenbosch, South Africa.

KenNel, R., 1972: Die Buchendurchforstungsversuche in Bayern von 1870 bis 1970 - Mit dem Modell einer Strukturertragstafel für die Buche. München: Forstl. Forschungsber. Nr. 7.

LEMM, R, 1991: Ein dynamisches Forstbetriebssimulationsmodell. Diss. Professur Forsteinr. u. Waldwachstum ETH Zürich.

MURRAY, D.M., and GADOW, K. V., 1991: Relationships between the diameter distributions before and after thinning. Forest Science 37(2): 552 - 559.

PreusSNER, K., 1974: Aufstellung und experimentelle Überprüfung mathematischer Modelle für die Entwicklung der Durchmesserverteilung von Fichtenbeständen. Diss. Tharandt.

RöMISCH, K., 1983: Ein mathematisches Modell zur Simulation von Wachstum und Durchforstung gleichaltriger Reinbstände. Diss. Tharandt.

Staupendahl, K., und Puumalainen, J., 1999: Modellierung des Einflusses von Durchforstungen auf die Durchmesserverteilung von gleichaltrigen Fichtenreinbeständen. Centralbl. f. ges. Forstw. 116 (4): 249 262.

The AsSOCIATION OF Forestry Research StATIONS, 1902: Beratungen der vom Verein Forstlicher Versuchsanstalten eingesetzten Kommission zur Feststellung des neuen Arbeitsplanes für Durchforstungs- und Lichtungsversuche. AFJZ 78: 180 - 184.

Wenk, G, Antanaitis, V., und Smelko, S., 1990: Waldertragslehre. Berlin, Dt. Landwirtschaftsverlag, 448 S. 



\section{LEBENSLAUF}

\section{KAI STAUPENDAHL}

Otto-Lauffer-Str. 13

37077 Göttingen

E-Mail: kstaupe@gwdg.de

Familienstand: Ledig

Geboren in Dortmund

$1975-1979$

$1979-1988$

Mai 1988

$1988-1989$

$1989-1990$

1990

Okt. 1990 - März 1991

$1991-1996$

Juni 1996

Aug. - Dez. 1996

Jan. 1997 - Aug. 2001

Dez. 2001

Seit Juni 2007

Besuch der Evangelischen Grundschule, Bad Lippspringe, und des Städtischen Gymnasiums Theodorianum, Paderborn Abitur

Grundwehrdienst in Wuppertal und Augustdorf, Nordrhein-Westfalen

Praktikum und Studium im Fach Flugzeugbau an der Fachhochschule Hamburg (2 Sem.)

Studium generale mit Schwerpunkt Philosophie an der Universität Hamburg (1 Sem.)

Forstliches Hochschulpraktikum im ehemaligen Staatl. Forstamt Hardegsen (Solling)

Studium der Forstwissenschaften an der Albert-Ludwigs-Universtät Freiburg und der Georg-August-Universität Göttingen

Abschluß als Dipl.-Forstwirt

Praktikum beim Forstlichen Dienstleister Skogssällskapets Förvaltning AB in Uppsala, Schweden, im Rahmen des EU-Programms Leonardo da Vinci

Wissenschaftlicher Mitarbeiter am Institut für Forsteinrichtung und Ertragskunde (heute: Abt. für Waldinventur und Fernerkundung bzw. - zusammen mit dem früheren Institut für Forstökonomie - Abt. für Forstökonomie und Forsteinrichtung im Burckhardt-Institut) der Georg-August-Universität Göttingen

Ausgründung des Planungsbüros ARGUS Forstplanung, seitdem Kooperationspartner für Forstliche Forschungsinstitutionen und Consultant für Forstplanung, Waldinventuren und Forstliche Informationssysteme (s. www.argus-forstplanung.de) Wissenschaftlicher Mitarbeiter an der Abt. für Forstökonomie und Forsteinrichtung, Universität Göttingen, im Rahmen des BMBFProjektes „Anpassungsstrategien für eine nachhaltige Waldbewirtschaftung unter sich wandelnden Klimabedingungen - Decision Support System Wald und Klimawandel (DSS-WuK)““ 